\title{
SECONDARY BUCKLING OF LAMINATED COMPOSITE PLATES
}

by

\author{
Nachiketa Tiwari
}

Dissertation submitted to the Faculty of Virginia Polytechnic Institute and State University in partial fulfillment of the requirements for the degree of

Doctor of Philosophy

in

Engineering Mechanics

Prof. Michael W. Hyer, Chairman

Prof. Eric R. Johnson

Prof. Liviu Librescu

Prof. Raymond H. Plaut

Prof. Saad A. Ragab

May 3, 1999

Virginia Polytechnic Institute and State University

Blacksburg, VA 24061

Keywords: secondary buckling, snap through, composites, imperfections, boundary conditions. Copyright 1999, Nachiketa Tiwari 


\title{
SECONDARY BUCKLING OF LAMINATED COMPOSITE PLATES
}

\author{
by \\ Nachiketa Tiwari \\ Michael W. Hyer, Chairman \\ Engineering Mechanics
}

\begin{abstract}
(ABSTRACT)
The postbuckling load carrying capacity of composite plates offers immense potential to their applications for loads exceeding their primary buckling load. However, such an efficient and economical usage of these plates can be reliable only if the nonlinear postbuckling behavior of these plates, which includes a good understanding of secondary buckling, is understood thoroughly. The present investigation is an attempt to understand secondary buckling of almost square composite clamped-simply supported plates, both unstiffened as well as stiffened, in some detail. With the help of the finite element method, a large number of numerical studies have been conducted to understand the secondary buckling characteristics. The sensitivity of these characteristics to variations in boundary conditions, lamination sequence, imperfections, and stiffener geometry has been considered. It has been found that the occurrence of secondary buckling in clamped-simply supported plates under uniform end shortening critically depends on the intensity of restrictions imposed on the inplane normal displacements along the unloaded simply supported edges of the plate. These restrictions could be due to the actual boundary conditions at these edges, or due to the presence of stiffeners along these edges. It has also been found that the presence of imperfections significantly delays the event of secondary buckling. Finally, it has been found that changes in lamination sequence of the plate alter its secondary buckling characteristics in ways that are, in general, quantitative in nature. The numerical investigations were followed by a limited number of experiments involving the testing of unstiffened as well as stiffened composite plates with the intent of augmenting the confidence in the numerical predictions made. Three different lamination sequences were considered during the testing phase of this investigation. It was found that the agreement between experimental data and numerical predictions was quite good. The occurrence of secondary buckling followed the predictions closely.
\end{abstract}




\section{ACKNOWLEDGEMENTS}

I take this opportunity to gratefully acknowledge the friendship, guidance, and support, both personal and technical, of my advisor, Professor Michael W. Hyer. I also take this chance to express my deepest sense of gratitude to Professors Eric Johnson, Liviu Librescu, Raymond Plaut, and Saad Ragab, for providing me with their much needed advice related to my academic endeavors. The partial financial support to this research from the NASA Langley Research Center, and the Center for Composite Materials and Structures at Virginia Tech is gratefully acknowledged.

During the course of my research work, a number of people offered their invaluable help to facilitate my work. Bob Simmonds and George Lough helped me use the Instron laboratory. Bill Shaver and Archie Montgomery of the ESM departmental machine shop went out of their way to finish my machining jobs quickly, despite their tight schedules. Danny Reed of the Composite Fabrication Lab department helped me a great deal in getting my laminates fabricated in time. Paula Davis, Beverly Williams, and Cindy Hopkins were of immense help to me in administrative matters. Duane Taylor and Tim Tomlin of the ESM Computer Lab were of considerable help in providing me all the requisite computational resources needed for my research. Thanks a lot to all of these gentlepersons. Without there invaluable help, my research work would not have borne its fruition.

I would also like to thank my parents, Ramakant and Jayanti Tiwari, and my wife Indira for all the

love and support that they have given me over the years. Last, but not the least, I would like to thank all of my teachers, starting from my pre-school days, whose faith in me made me achieve whatever little I have done to this date. 


\section{TABLE OF CONTENTS}

1.1 Overview 1

1.1.1 Secondary Buckling $\quad 1$

1.2 Review of Past Research Work 3

1.2.1 Review of Past Research Concerning Seondary Buckling 4

1.3 Comments and Discussion 9

$1.4 \quad$ A Road Map to Remaining Chapters 11

$2 \quad$ Finite Element Formulation and Validation $\quad 14$

2.1 Nonlinear Shear Deformation Theory 14

2.1.1 Geometry 14

2.1.2 Strain-Displacement Relations 15

$\begin{array}{lll}\text { 2.1.3 Variational Statement } & 17\end{array}$

$\begin{array}{lll}2.1 .4 & \text { Constitutive Laws } & 18\end{array}$

2.2 Finite Element Formulation 19

2.2.1 Imposition of Constraint Equations 22

2.3 Stability Considerations and Bifurcation Analysis 24

$2.4 \quad$ Validation of the Finite Element Code 26

2.4.1 Unsymmetrically Laminated Linear Rectangular Composite Plate $\begin{array}{ll}\text { Under Uniform End Compression } & 27\end{array}$

2.4.2 Isotropic Plates Subjected to Uniform Lateral Pressure 28

2.4.2.1 Simply Supported Isotropic Square Plate 28

2.4.2.2 Loosely Clamped Isotropic Square Plate 30

2.4.2.3 Rigidly Clamped Isotropic Rectangular Plate 31

2.4.3 Symmetric Composite Plates Subjected to Uniform Lateral Pressure 32

2.4.3.1 Rigidly Clamped Orthotropic Composite Plate Under Uniform Lateral Pressure 
2.4.3.2 Loosely Clamped Anisotropic Unsymmetrically Laminated Composite Plate Subjected to Uniform Lateral Pressure

2.4.4 Unsymmetrically Laminated Linear Plate Subjected to Uniform Temperature Change 35

$\begin{array}{lll}2.5 & \text { Closure } & 36\end{array}$

$\begin{array}{lll}3 & \text { Numerical Predictions for Secondary Buckling } & 37\end{array}$

$\begin{array}{lll}3.1 & \text { Introduction } & 37\end{array}$

3.2 Details of Clamped-Simply Supported Plates Studied 37

3.3 Influence of Boundary Conditions and Stiffener Thickness on the Behavior of Quasi-Isotropic Plates 41

3.3.1 Unstiffened Quasi-Isotropic Plates 41

3.3.2 Stiffened Quasi-Isotropic Plates 45

3.4 Influence of Lamination Sequence 47

3.4.1 Role of Lamination Sequence on the Response of Unstiffened Panels 48

3.4.2 Role of Imperfections on the Response of Quasi-Isotropic Panels 49

3.4.2.1 Unstiffened Plates 53

3.4.2.2 Stiffened Plates $\quad 53$

3.5 Interaction of Lamination Sequence and Stiffener Thickness 54

$\begin{array}{lll}3.6 & \text { Metallic Plates }\end{array}$

3.7 Influence of Out-of-Plane Shear Deformations on Secondary Buckling 59

$\begin{array}{lll}3.8 & \text { Closure } & 60\end{array}$

$4 \quad$ Description of Experiments $\quad 63$

$\begin{array}{lll}4.1 & \text { Introduction } & 63\end{array}$

$\begin{array}{lll}4.2 & \text { Test Fixture and Setup } & 63\end{array}$

4.3 Fabrication of Composite Plates 69

$\begin{array}{lll}4.4 & \text { Closure } & 70\end{array}$ 
$5 \quad$ Comparison of Experimental and Numerical Data $\quad 71$

$\begin{array}{lll}5.1 & \text { Material Characterization } & 71\end{array}$

$\begin{array}{lll}5.1 .1 & {[90]_{4} \text { Test Coupon }} & 71\end{array}$

$\begin{array}{lll}5.1 .2[0]_{4} \text { Test Coupon } & 72\end{array}$

5.1.3 $\left[90_{2} / 0 / 90 / 0_{1 / 2}\right]_{\mathrm{S}}$ Test Coupon $\quad 72$

$\begin{array}{lll}5.1 .4 & {[ \pm 45]_{\mathrm{S}} \text { Test Coupon }} & 72\end{array}$

5.2 Experimental Results for Plates Subjected to Compression 75

5.2.1 The $[ \pm 45 / 0 / 90]_{\mathrm{S}}$ Plates 76

5.2.1.1 The $[ \pm 45 / 0 / 90]_{\mathrm{S}}$ Unstiffened Plate 76

5.2.1.2 The $[ \pm 45 / 0 / 90]_{\mathrm{S}}$ Stiffened Plate $\quad 81$

$\begin{array}{lll}\text { 5.2.2 The }\left[ \pm 45 / 0_{2}\right]_{\mathrm{S}} \text { Plates } & 87\end{array}$

5.2.2.1 The $\left[ \pm 45 / 0_{2}\right]_{\mathrm{S}}$ Unstiffened Plate $\quad 87$

5.2.2.2 The $\left[ \pm 45 / 0_{2}\right]_{\mathrm{S}}$ Stiffened Plate $\quad 91$

5.2.3 The $\left[ \pm 45 / 90_{2}\right]_{\mathrm{S}}$ Plates 96

5.2.3.1 The $\left[ \pm 45 / 90_{2}\right]_{\mathrm{S}}$ Unstiffened Plate 96

$\begin{array}{lll}\text { 5.2.3.2 The }\left[ \pm 45 / 90_{2}\right]_{\mathrm{S}} \text { Stiffened Plate } & 100\end{array}$

$\begin{array}{lll}5.3 & \text { Closure } & 105\end{array}$

$6 \quad$ Conclusions and Recommendations 109

$\begin{array}{lll}6.1 & \text { Introduction } & 109\end{array}$

6.2 Summary of Conclusions of Numerical Studies 110

6.2.1 Influence of Boundary Conditions 111

6.2.2 Influence of Stiffeners 111

6.2.3 Influence of Lamination Sequence 112

6.2.4 Influence of Imperfections 112

6.2.5 Comparison with Metallic Plates 113

6.2.6 Influence of Out-of-Plane Shear Deformations on Secondary Buckling 113

6.3 Summary of Conclusions of Experimental Studies 113

6.4 Scope for Further Research 114 
8 Appendix: Expressions for Direct Stiffness Matrices

119 


\section{LIST OF FIGURES}

\section{$1 \quad$ Introduction}

Fig. 1.1 Concept of Secondary Buckling 2

Fig. 1.1-a A Perfectly Flat Plate Subjected to External Force $P \quad 2$

Fig. 1.1-b Load-End Shortening Response of a Perfectly Flat Plate 2

Fig. 1.1-c Plate Postbuckling Configuration before Secondary Buckling 2

Fig. 1.1-d Plate Postbuckling Configuration after Secondary Buckling 2

\section{$2 \quad$ Finite-Element Formulation and Validation}

Fig. 2.1 Plate Under Uniform Compression 15

Fig. 2.2 Bifurcation Point 25

Fig. 2.3 Unsymmetrically Laminated Linear Plate Under Uniform End Compression 27

Fig. 2.4 Details of a Simply Supported Isotropic Square Plate under Uniform Pressure 29

Fig. 2.5 Normalized Plot of Central Deflection versus Uniform Pressure for Simply Supported Isotropic Square Plate

Fig. 2.6 Details of a Loosely Clamped Isotropic Square Plate Under Uniform Pressure 30

Fig. 2.7 Normalized Plot of Central Deflection versus Uniform Pressure for Loosely Clamped Isotropic Square Plate

Fig. 2.8 Details of a Rigidly Clamped Isotropic Rectangular Plate under Uniform Pressure

Fig. 2.9 Normalized Plot of Central Deflection versus Uniform Pressure for Rigidly Clamped Isotropic Rectangular Plates 32

Fig. 2.10 Rigidly Clamped Orthotropic Plate Subjected to Uniform Pressure 33

Fig. 2.11 Normalized Plot of Central Deflection versus Uniform Pressure for Rigidly Clamped Orthotropic Plates

Fig. 2.12 Normalized Plot of Central Deflection versus Uniform Pressure for Loosely Clamped Unsymmetric Square Plates

Fig. 2.13 Unsymmetrically Laminated Plate Subjected to Uniform Temperature Change 35 
Fig. 3.1 Details of the Plate Subjected to Analysis 38

Fig. 3.2a Postbuckling Plate Configuration before Secondary Buckling 43

Fig. 3.2b Postbuckling Plate Configuration after Secondary Buckling 43

Fig. 3.3 Deflection Response of Perfectly Flat Unstiffened Plates 43

Fig. 3.4 Load Response of Perfectly Flat Unstiffened Plates 43

Fig. 3.5 Deflection Response of Perfectly Flat Stiffened Plates 43

Fig. 3.6 Load Response of Perfectly Flat Stiffened Plates 43

Fig. 3.7 Load Sharing between Stiffeners and Plates 44

Fig. 3.8a Illustration of $v$ displacement which has been plotted in Figs. 3.8b. 3.8c 44

Fig. 3.8b Variation of $v$ Displacement prior to Secondary Buckling 44

Fig. 3.8c Variation of $v$ Displacement after Secondary Buckling 44

Fig. 3.9a Configuration of Stiffened Plate before Secondary Buckling 44

Fig. 3.9b Configuration of Stiffened Plate after Secondary Buckling 44

Fig. 3.10 Deflection Response of Perfect Plates with Fixed SS Edges 51

Fig. 3.11 Load Response of Perfect Plates with Fixed SS Edges 51

Fig. 3.12 Deflection Response of Perfect Plates with Straight SS Edges 51

Fig. 3.13 Load Response of Perfect Plates with Straight SS Edges 51

Fig. 3.14 Deflection Response of Perfect Plates with Unconstrained SS Edges 51

Fig. 3.15 Load Response of Perfect Plates with Unconstrained SS Edges 51

Fig. 3.16a Imperfections in Unstiffened Quasi-isotropic Plate 52

Fig. 3.16b Imperfections in Stiffened Quasi-isotropic Plate $(t=2.0 \mathrm{~mm}) \quad 52$

Fig. 3.17 Deflection Response of Imperfect Quasi-isotropic Unstiffened Plates 52

Fig. 3.18 Load Response of Imperfect Quasi-isotropic Unstiffened Plates 52

Fig. 3.19 Deflection Response of Imperfect Quasi-isotropic Stiffened Plates 52

Fig. 3.20 Load Response of Imperfect Quasi-isotropic Stiffened Plates 52

Fig. 3.21 Deflection Response of Perfectly Flat Stiffened Plates $(t=2.0 \mathrm{~mm}) \quad 55$

Fig. 3.22 Load Response of Perfectly Flat Stiffened Plates $(t=2.0 \mathrm{~mm}) \quad 55$

Fig. 3.23 Deflection Response of Perfectly Flat Stiffened Plates $(t=1.8 \mathrm{~mm}) \quad 55$

Fig. 3.24 Load Response of Perfectly Flat Stiffened Plates $(t=1.8 \mathrm{~mm})$

Fig. 3.25 Deflection Response of Perfectly Flat Stiffened Plates $(t=1.6 \mathrm{~mm})$ 
Fig. 3.26 Load Response of Perfectly Flat Stiffened Plates $(t=1.6 \mathrm{~mm})$

Fig. 3.27 Deflection Response of Perfectly Flat Stiffened Plates $(t=1.4 \mathrm{~mm}) \quad 56$

Fig. 3.28 Load Response of Perfectly Flat Stiffened Plates $(t=1.4 \mathrm{~mm}) \quad 56$

Fig. 3.29 Deflection Response of Perfectly Flat Stiffened Plates $(t=1.2 \mathrm{~mm}) \quad 56$

Fig. 3.30 Load Response of Perfectly Flat Stiffened Plates $(t=1.2 \mathrm{~mm}) \quad 56$

Fig. 3.31 Deflection Response of Imperfect and Stiffened Plates $(t=2.0 \mathrm{~mm}) \quad 56$

Fig. 3.32 Load Response of Imperfect and Stiffened Plates $(t=2.0 \mathrm{~mm}) \quad 56$

Fig. 3.33 Deflection Response of Unstiffened Quasi-isotropic and Metallic Plates 61

Fig. 3.34 Load Response of Unstiffened Quasi-isotropic and Metallic Plates 61

Fig. 3.35 Impact of Out-of-Plane Shear Stiffness on Deflection Response 61

Fig. 3.36 Impact of Out-of-Plane Shear Stiffness on Load Response 61

Fig. 3.37 Variation of $\psi^{\mathrm{o}}{ }_{x}$ and $\partial w / \partial x$ along Vertical Mid-axis of Plate 61

Fig. 3.38 Variation of Shear Strain $\gamma_{z x}^{0}$ along Vertical Mid-axis of Plate 61

Fig. 3.39 Variation of $\psi_{y}^{0}$ and $\partial w / \partial y$ along Horizontal Mid-axis of Plate 62

Fig. 3.40 Variation of Shear Strain $\gamma_{z y}^{0}$ along Horizontal Mid-axis of Plate 62

\section{Description of Experiments}

Fig. 4.1 Schematic Diagram of the Fixture with Plate 64

Fig. 4.2 Location of Strain Gages and DCDTs on Unstiffened Plates as seen from the Front Side

Fig. 4.3 Location of Strain Gages and DCDTs on Stiffened Plates as seen from the Front side

Fig. 4.4 Schematic Representation of Instrumentation Associated with the Measurement of Out-of Plane Deflections of an Unstiffened Plate

Fig. 4.5 Equipment Layout for Experimental Test as seen from the Side

Fig. 4.6 Prescription of Heat and Pressure During the Curing of BMI Laminate 


\section{Comparison of Experimental Results with Numerical Predictions}

Fig. 5.1 Details of the Test Coupon $\quad 71$

Fig. 5.2 Stress-Strain Response for the $\left[90_{4}\right]$ Laminate 74

Fig. 5.3 Poisson-Strain Response for the $\left[0_{4}\right]$ Laminate 74

Fig. 5.4 Stress-Strain Response for the $\left[90_{2} / 0 / 90 / 0_{1 / 2}\right]_{\mathrm{S}}$ Laminate 74

Fig. 5.5 $\bar{\sigma}_{x} / 2$ versus $\left(\varepsilon^{o}{ }_{x}-\varepsilon^{o}{ }_{y}\right)$ for the $[ \pm 45]_{S}$ Laminate $\quad 74$

Fig. 5.6 Midplane Strains in Unstiffened $[ \pm 45 / 0 / 90]_{S}$ Plate 79

Fig. 5.7 Bending Strains in Unstiffened $[ \pm 45 / 0 / 90]_{S}$ Plate 79

Fig. 5.8 End Shortening Response for Unstiffened $[ \pm 45 / 0 / 90]_{S}$ Plate 80

Fig. 5.9 Deflection Response for Unstiffened $[ \pm 45 / 0 / 90]_{S}$ Plate 80

Fig. 5.10 Midplane Strains in Stiffened $[ \pm 45 / 0 / 90]_{S}$ Plate $\quad 84$

Fig. 5.11 Bending Strains in Top Half of Stiffened $[ \pm 45 / 0 / 90]_{S}$ Plate 84

Fig. 5.12 Bending Strains in Bottom Half of Stiffened $[ \pm 45 / 0 / 90]_{S}$ Plate 85

Fig. 5.13 End Shortening Response for Stiffened $[ \pm 45 / 0 / 90]_{S}$ Plate 85

Fig. 5.14 Out-of-Plane Deflection Response for Stiffened $[ \pm 45 / 0 / 90]_{\mathrm{S}}$ Plate 86

Fig. 5.15 Postbuckling Moire' Fringe Patterns for Out-of-Plane Deflections in Stiffened $[ \pm 45 / 0 / 90]_{\mathrm{S}} \mathrm{Plate} \quad 86$

Fig. 5.16 Secondary Buckling Moire' Fringe Patterns for Out-of-Plane Deflections in $\begin{array}{ll}\text { Stiffened }[ \pm 45 / 0 / 90]_{S} \text { Plate } & 87\end{array}$

Fig. 5.17 Midplane Strains in Unstiffened $\left[ \pm 45 / 0_{2}\right]_{\mathrm{S}}$ Plate 89

Fig. 5.18 Bending Strains in Unstiffened $\left[ \pm 45 / 0_{2}\right]_{\mathrm{S}}$ Plate 89

Fig. 5.19 End Shortening Response for Unstiffened $\left[ \pm 45 / 0_{2}\right]_{\text {s }}$ Plate 90

Fig. 5.20 Deflection Response for Unstiffened $\left[ \pm 45 / 0_{2}\right]_{\mathrm{S}}$ Plate 90

Fig. 5.21 Midplane Strains in Stiffened $\left[ \pm 45 / 0_{2}\right]_{\mathrm{s}}$ Plate 93

Fig. 5.22 Bending Strains in Top Half of Stiffened $\left[ \pm 45 / 0_{2}\right]_{\mathrm{s}}$ Plate 93

Fig. 5.23 End Shortening Response for Stiffened $\left[ \pm 45 / 0_{2}\right]_{\mathrm{S}}$ Plate 94

Fig. 5.24 Out-of-Plane Deflection Response for Stiffened $\left[ \pm 45 / 0_{2}\right]_{\mathrm{S}}$ Plate 94 
Fig. 5.25 Postbuckling Moire' Fringe Patterns for Out-of-Plane Deflections in Stiffened $\left[ \pm 45 / 0_{2}\right]_{\mathrm{S}}$ Plate

Fig. 5.26 Secondary Buckling Moire' Fringe Patterns for Out-of-Plane Deflections in $\begin{array}{ll}\text { Stiffened }\left[ \pm 45 / 0_{2}\right]_{\mathrm{S}} \text { Plate } & 95\end{array}$

Fig. 5.27 Midplane Strains in Unstiffened $\left[ \pm 45 / 90_{2}\right]_{\mathrm{s}}$ Plate 98

Fig. 5.28 Bending Strains in Unstiffened $\left[ \pm 45 / 90_{2}\right]_{\mathrm{S}}$ Plate 98

Fig. 5.29 End Shortening Response for Unstiffened $\left[ \pm 45 / 90_{2}\right]_{\mathrm{S}}$ Plate 99

Fig. 5.30 Deflection Response for Unstiffened $\left[ \pm 45 / 90_{2}\right]_{\mathrm{S}}$ Plate 99

Fig. 5.31 Midplane Strains in Stiffened $\left[ \pm 45 / 90_{2}\right]_{\mathrm{S}}$ Plate 102

Fig. 5.32 Bending Strains in Stiffened $\left[ \pm 45 / 90_{2}\right]_{\mathrm{S}}$ Plate 102

Fig. 5.33 End Shortening Response for Stiffened $\left[ \pm 45 / 90_{2}\right]_{\mathrm{S}}$ Plate 103

Fig. 5.34 Deflection Response for Stiffened $\left[ \pm 45 / 90_{2}\right]_{\mathrm{s}}$ Plate 103

Fig. 5.35 Postbuckling Moire' Fringe Patterns for Out-of-Plane Deflections in Stiffened $\left[ \pm 45 / 90_{2}\right]_{\mathrm{S}}$ Plate

Fig. 5.36 Secondary Buckling Moire' Fringe Patterns for Out-of-Plane Deflections in Stiffened $\left[ \pm 45 / 90_{2}\right]_{S}$ Plate 


\section{LIST OF TABLES}

Table 2.1 Comparison of FEM and Exact Results for an Unsymmetrically Laminated Linear Plate

Table 2.2 FEM and Exact Results for an Unsymmetrically Laminated Plate Subjected to Uniform Temperature Field

Table 3.1 Nomenclature of Perfectly Flat Plates

Table 3.2 Nomenclature of Plates with Out-of-Plane Imperfections

Table 3.3 Nomenclature of Metallic Plates and Out-of-Plane Shear Stiff Plates

Table 3.4 Case Studied to Explore the Role of Lamination Sequence on Postbuckling Response of Unstiffened Plates

Table 3.5 Case Studied to Explore the Role of Imperfections on Postbuckling Response of Unstiffened and Stiffened Plates

Table 3.6 Case Studied to Understand the Interaction between Layup and Stiffener Thickness and their Influence on Postbuckling

Table 4.1 Position (mm) of Strain Gages and DCDTs on Unstiffened Plates

Table 4.2 Position (mm) of Strain Gages and DCDTs on Stiffened Plates

Table 5.1 Details of Plate Used for Testing

Table 5.2 Comparison of Experimental and Numerical Data for the Plates Tested 


\section{INTRODUCTION}

\subsection{OVERVIEW}

Thin plates have been extensively used in a variety of structural applications. Quite often, plates are designed to perform under compressive loading conditions. Hence, buckling and postbuckling of plates has always been an interesting research field among structural engineers. Unlike beams and bars, plates can have a significant amount of load carrying capacity in the postbuckling range. Consequently, plates are often designed to bear compressive loads which exceed the primary buckling load by several factors. Significant amount of attention has been paid to the postbuckling characteristics of thin plates; specifically, load-deflection relations, deflected shapes, and maximum stress levels.

Experiments on plates have quite often revealed that the postbuckling shape of a plate can suddenly change if the applied compressive load exceeds a certain threshold. This sudden change of the postbuckled shape is essentially a dynamic event, and is often referred as "snap-through". Alternatively, it has also been termed "secondary buckling", "mode jumping" and "secondary bifurcation".

\subsubsection{Secondary Buckling}

Figure 1.1-a depicts an almost square, perfectly flat plate subjected to a uniform compressive inplane end shortening displacement, $\Delta$. Any particular edge can be clamped or simply supported. Note the $x-y-z$ coordinate system, where the $x$ axis corresponds to the loading axis. Edges, identified as $1,2,3$, and 4, will be discussed shortly. The consequential inplane compressive force is $P$. Figure 1.1-b is a depiction of the load-end shortening response of this plate. With increasing load from the zero load condition, the plate exhibits only inplane deformation until the buckling point is reached. This response of the plate, known as the prebuckling response, is shown by line O-A in Fig. 1.1-b. With further increment in end compression, the plate buckles and enters into the postbuckling range. The response in this range is given by line $\mathrm{A}-\mathrm{B}$, and the shape of the plate is shown in Fig. 1.1-c. One noteworthy feature of this postbuckled out-of-plane configuration is that 
the plate has one half-wave in both the $x$ and the $y$ directions.

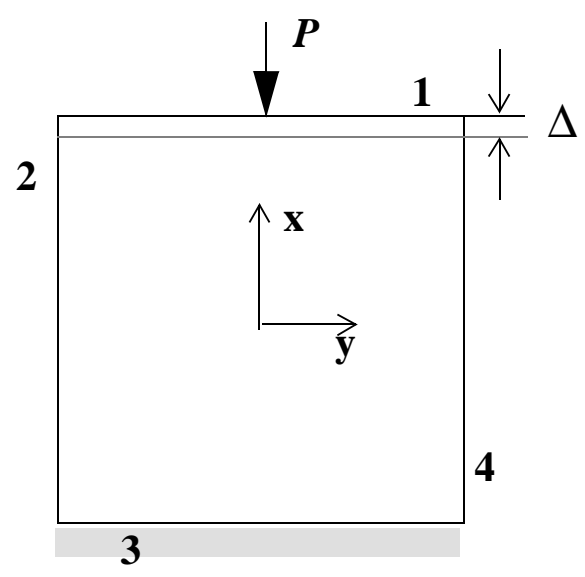

Fig. 1.1-a: A Perfectly Flat Plate Subjected to External Force $P$

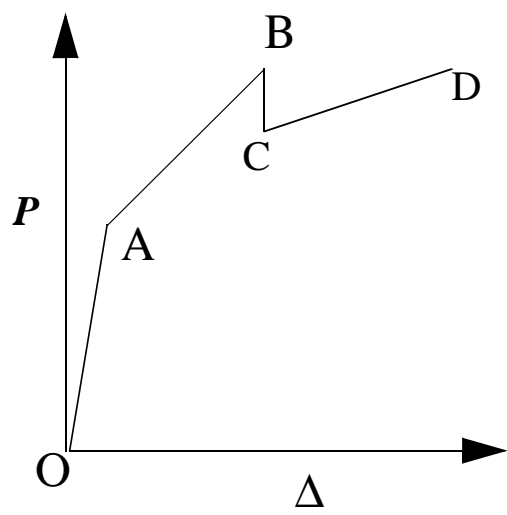

Fig. 1.1-b: Load-End Shortening Response of a Perfectly Flat Plate

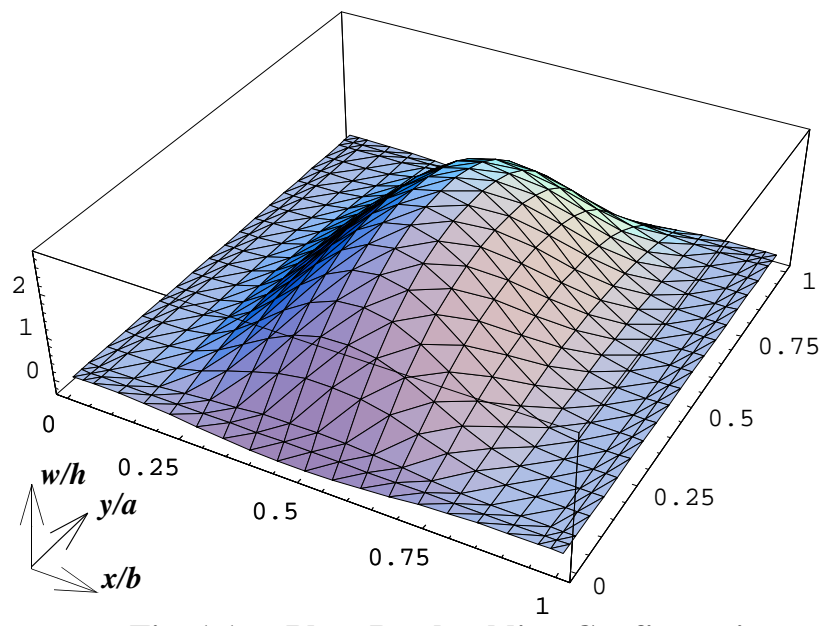

Fig. 1.1-c: Plate Postbuckling Configuration before Secondary Buckling

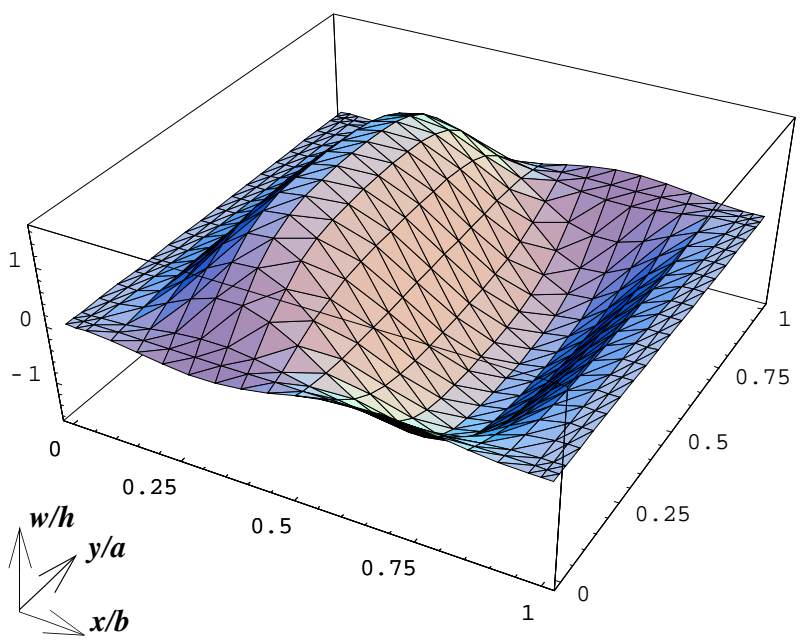

Fig. 1.1-d: Plate Postbuckling Configuration after Secondary Buckling

Fig. 1.1: Concept of Secondary Buckling

If the plate is compressed further into the deep postbuckling range, under certain conditions the configuration suddenly changes. This sudden change is associated with a drop in the load carried by the plate, which is shown in Fig. 1.1-b by line B-C. The sudden transition in the configuration is termed secondary buckling, and a typical out-of-plane configuration of the plate is shown in Fig. 1.1-d. The configuration has two half-waves in the loading $(x)$ direction, and one half-wave in the transverse (y) direction. Upon further compression, the load increases again with increasing end shortening. Line C-D in Fig. 1.1-b is representative of the post-secondary buckling load-end 
shortening response.

To be mathematically precise, the terms "secondary buckling" and "secondary bifurcation" are accurate labels of this phenomenon only when the physical structure has no imperfections and has no bending-extension coupling stiffness coefficients. This is so, since there is no well-defined and sharp primary buckling point in load-end shortening response relation for most structures having imperfections in the out-of-plane direction, or having non-zero bending-extension coupling coefficients.

Secondary buckling of a structure under compression is quite undesirable. It can lead to a sudden rise in the stress levels, initiating failure of the material. Hence, any structural design of a plate which seeks to utilize postbuckling strength and stiffness for purposes of increased economy and weight saving should explore the possibility of a secondary buckling event, since it is quite possible that secondary buckling may define the upper bound of the operational range of the plate. It is for this reason that a thorough understanding of the secondary buckling phenomenon is required. In the following section a brief review of past research conducted in this field is presented.

\subsection{REVIEW OF PAST RESEARCH WORK}

Bryan [1] was perhaps the first researcher to conduct the buckling analysis of isotropic plates. A similar analysis for plates made from anisotropic materials was conducted by March [2] in 1942. With the emergence of tailored composite materials, a significant amount of work has been done which is specific to the behavior of composite structures. Leissa's review paper [3] is a comprehensive record of studies concerning the buckling phenomenon in structures made from composite materials. Considerable amount of work regarding buckling and postbuckling of thermomechanically loaded composite plates is covered in references [4] through [6]. Librescu, Lin, Nemeth, and Starnes [4] have shown that the degree of tangential edge constraints of a plate has a bearing on the imperfection sensitivity of a thermally loaded curved plate. Their work has also captured the role played by shear deformations in this regard. Librescu et al. [4], and Librescu and Lin [5] have also shown that the onset of primary static instability, i.e. buckling, occurs at an early stage as the severity of tangential restraints on edges of a thermally loaded com- 
posite plate is increased. A comprehensive summary of diverse issues concerning thermomechanical buckling of curved as well as flat composite plates has been provided by Librescu in [6].

\subsubsection{Review of Past Research Concerning Secondary Buckling}

In 1958, Stein [7,8] made an attempt to understand the phenomenon of secondary buckling. His approach was that of constructing a relatively simple mathematical model which could buckle in more than one mode. Thus, his model had three one-dimensional elements which were connected with linear torsional springs. The structure was simply supported on both ends. Since secondary buckling was suspected to be a nonlinear phenomenon, Stein restrained the lateral deflection of his three-link model by using three lateral nonlinear extensional springs. The stiffnesses of these springs were not the same. Both controlled loading and controlled end shortening boundary conditions were considered. By manipulating the relative magnitudes of the stiffnesses of the lateral nonlinear springs, Stein arrived at the following conclusions:

- There are three postbuckled solutions for the three-element column.

- Two of these solutions are uncoupled, while the third one is coupled in the sense that is a linear combination of the two uncoupled modes. The coupled solution acts as a path of transition from one buckled state to another one.

- The stability of this coupled path is a function of the relative stiffnesses of the three nonlinear extensional springs.

In another study [9], Stein analyzed an infinite length isotropic plate strip subjected to compressive loads. The sides of the plate were assumed to be simply supported. Stein's analysis showed that the plate's lowest buckling load corresponded to a longitudinal half-wavelength equalling the width of the plate. Stein observed that an increase in the compressive load beyond this critical number gradually decreased the half-wavelength of the out-of-plane deflections. Past work in the field of buckling of plates has revealed that a plate with finite dimensions can only buckle into shapes whose half-wavelengths are integral factors of the plate dimensions. Because of this, the 
buckled patterns of plates with finite dimensions cannot gradually decrease, despite the tendency of the half-wavelength to shorten. In view of these restrictions, it has been postulated that when the compressive loads on a finite plate exceed a certain limit, secondary buckling occurs.

The issues raised by Stein were later analyzed in greater detail by Supple [10, 11, 12]. Supple [10] began his studies in a very systematic and generic way by using the potential energy of a conservative elastic structure as the stability criterion. Assuming potential energy as a function of load and two out-of-plane deflection functions, $u_{1}$ and $u_{2}$, Supple classified the structural systems under consideration into four categories. Here, $u_{1}$ and $u_{2}$ are postbuckling equilibrium shapes associated with the lowest buckling load and the next higher buckling load, respectively. The basis of this classification were functions involving higher-order derivatives of potential energy, and a parameter $\Delta p$, which is defined as the difference between the first two buckling loads of the structure. His conclusions have been summarized below:

- Any structural system whose first two uncoupled postbuckling equilibrium paths, $u_{l}$ and $u_{2}$, are stable will also have a coupled postbuckling equilibrium path. This coupled path will be stable if

$$
V,{ }_{1111} V, 2222>(3 V, 1122)^{2}
$$

Here, $V$ is the potential energy function, and $V,_{i}$ is its partial derivative with respect to $u_{i}$.

- Further, if the inequality relation given above is not satisfied, the coupled postbuckling equilibrium path will be unstable. In such a case, there will be no change in the buckle patterns of the plate.

Supple's analysis has limited implications for the following reasons:

- It is quite difficult to evaluate the derivatives of potential energy function with respect to uncoupled postbuckling mode shapes, $u_{1}$ and $u_{2}$. 
- Supple's analysis is valid only for small values of $\Delta p$.

- This approach does not consider the contribution of inplane displacements to the potential energy function.

As an extension to his work in [10], Supple has also discussed the influence of imperfections on the change of buckle patterns in elastic structures. This has been done in [11]. Essentially, Supple uses a similar approach as in [12], with the modification that two small initial imperfections, $\varepsilon_{1}$ and $\varepsilon_{2}$, associated with $u_{1}$ and $u_{2}$, respectively, have also been considered in the definition of $V$. Supple's key conclusions have been listed as follows:

- An imperfection of the type $\varepsilon_{l}$ does not significantly alter the postbuckling behavior of structures which are not imperfection sensitive.

- Perfect structures exhibiting secondary buckling also exhibit a similar phenomenon in the presence of an $\varepsilon_{l}$ type of imperfection.

- If the imperfection is purely of type $\varepsilon_{2}$, then under certain conditions, the structure, which is in primary postbuckled state $u_{2}$, can snap at a higher load to state $u_{l}$, which is the lower uncoupled mode for the ideal system.

In [12], Supple has investigated the change in buckle patterns of a simply supported rectangular isotropic plate with an aspect ratio of 2. Using the stress function approach and a two-term series representations for out-of-plane displacement $w$, and imperfection $w_{o}$, Supple concluded that the secondary buckling of the plate was dependent on the magnitudes and the shapes of imperfections.

Using the dynamical criterion for structural stability as proposed by Movchan [13], Stroebel and Warner [14] have approached the issue of secondary buckling in isotropic von Karman plates. Liapunov's theorems were used to verify the stability of post-primary-buckling and post-secondary-buckling configurations of the plate. The post-secondary-buckling equilibrium solution was 
evaluated with the aid of Koiter-Fitch bifurcation analysis [8, 15, 16]. Following such an approach, these investigators were able to compute the secondary bifurcation loads for simply supported plates, with constant inplane displacements on its four edges, for varying aspect ratios.

Unlike several other investigators [12, 17, 18], Uemura and Byon [19] used more than two terms in their double Fourier series representations for the out-of-plane displacements while calculating secondary buckling loads for simply supported plates with an aspect ratio of unity. The authors used the positive definiteness of the second variation of potential energy as the criterion for system's stability. The secondary buckling load was calculated by equating the first variation of second variation of potential energy to zero. Using such an approach, the authors investigated the influence of imperfections and inplane boundary conditions on the values of secondary buckling loads. However, their approach is mathematically intensive, and cannot be used easily for more complicated boundary conditions and geometries. It is interesting to note that the values of secondary buckling loads as predicted by Uemura and Byon do not coincide with those made by Stroebel and Warner[14].

In another of their papers [20], Uemura and Byon approached the issue of secondary buckling in isotropic plates with clamped boundary conditions. They used the finite-element method to solve the problem. Their numerical investigations regarding the secondary buckling load agreed to some extent with their experimental results. However, the authors were not able to clearly identify the boundary conditions present in their in experimental investigations.

Nakamura and Uetani [17] approached the issue of secondary buckling by assuming series expansions for the unknown field variables. Their analysis was confined to plates which exhibit primary buckling in a multi-half-wave mode. Consequently, their conclusions cannot be used for plates of square shape. Potential energy considerations were employed for purposes of computation of secondary buckling load, and to ensure the stability of equilibrium configuration throughout the loading path. Their conclusions are:

- Inclusion of more than two terms in the series representations of out-of-plane displacements is very important for the accurate prediction of post-primary-buckling solution, 
for the computation of secondary buckling load, and for finding the post-secondarybuckling plate configuration.

- The number of terms to be used in a series solution can be determined in a consistent way.

- Secondary buckling is possible in simply supported plates which have constant inplane displacements on their edges, and whose aspect ratios lie between 1.4 and 8 .

Carnoy [21] and Hughes and Liu [22, 23] have conducted finite-element studies to predict the secondary buckling loads of square, isotropic plates with clamped edges. Using the finite-element code developed by Hughes, Hughes and Liu [22, 23] conclude that the values of secondary buckling loads are strongly dependent on the shape of imperfections in the plates. The finite-element results do not have good agreement with their experimental data.

Secondary buckling in plates made of composite materials have been investigated by Shin, Griffin, and Gurdal [24] and Stoll [25]. Shin et al. employed the series solution approach for the computation of secondary buckling loads in rectangular plates with simply supported edges. The inplane displacements normal to the edges were assumed to be constant along the edges. The authors investigated plates with varying degrees of orthotropy. The influence of bending-twisting coupling terms, $D_{16}$ and $D_{26}$, was neglected on account of their smallness in comparison to $D_{66}$. The potential energy criterion was use to detect the onset of secondary buckling. The authors have claimed that imperfections tend to delay the mode change.

Stoll [25] has solved the problem of rectangular orthotropic plates by using a series approximation for the out-of-plane displacement $w$. The plates were simply supported along the unloaded edges, which were parallel to the load, and clamped along the loaded edges. Each term in his series is a product of an unknown constant $q_{i}$ and the eigenfunction $w_{i}$ associated with the $i^{\text {th }}$ buckling mode. The buckling eigenfunctions are assumed to be products of sinusoidal functions in the direction normal to the simply supported edges, and unknown functions $\varnothing_{i}$, which vary only along the direction of loading. This is essentially the same approach as used by Whitney [26] 
to compute the buckling load of a clamped-simply supported plate. Such an assumed displacement field reduces the partial differential equilibrium equation for the out-of-plane direction to an ordinary differential equation in $\varnothing_{\mathrm{i}}$. From this equation a set of mutually orthogonal buckling eigenfunctions, which satisfy the essential boundary conditions, can be found. These eigenfunctions are later used to find a series solution for postbuckled state of the plate. Using such an approach, Stoll has computed the secondary buckling loads of a clamped-simply supported isotropic plate with a large aspect ratio. The author has claimed that imperfections, in general, tend to reduce the secondary buckling load.

Riks, Rankin, and Brogan [27] have conducted investigations into the suitability of using a mixed approach with a view to capture the state of the plate when it switches its shape at the onset of sec-

ondary buckling. Their approach essentially assumes the system to be quasi-static till the onset of secondary buckling and then uses a dynamic mathematical model to capture the plates shift from a primary buckled state to a secondary buckled state. Though comprehensive, this approach is numerically intensive, especially in the vicinity of a secondary buckling event.

Chang-jun and Xin-chun [28] have built upon Stein's work by developing analytical solutions for a simply supported von-Karman plate on a nonlinear elastic foundation. They have concluded that in such a system secondary buckling is triggered due to the nonlinear nature of the elastic foundation. However, many plates, as seen through experimental, numerical, and analytical data, also exhibit secondary buckling in the absence of such a nonlinear foundation.

\subsection{COMMENTS AND DISCUSSION}

An overview of the available literature concerning the secondary buckling phenomenon reveals the following:

1. The influence of boundary conditions on the secondary buckling phenomenon remains largely unresolved. Review of past literature reveals that almost all analyses, with the exception of $[19,20]$, have considered plates where the inplane displacements normal to the edges are constant. Uemura and Byon [19] have also explored the boundary condition where the inplane 
displacement normal to the loading direction, $v$, is fully unrestrained. Their results for an isotropic, simply supported plate, with an aspect ratio of unity, reveal that the magnitude of secondary buckling load is maximum for the plate with $v=0$ on edges. This load is minimum for a similar plate which has no constraints on $v$ on its edges. No experimental evidence is presented to support these contentions. The authors have also calculated secondary buckling loads for clamped plates by using the finite-element method. Limited agreement is seen between their numerical results and experimental data. Further, the authors have expressed doubts on the accuracy of the simulation of the boundary condition, $N_{y}=0$, in their experiments.

The boundary conditions $v=0$ and $N_{y}=0$ along the simply supported edges are two extreme cases. An intermediate boundary condition, lying between these two extremes, could be $v=$ constant along with the $\int N_{y} d x=0$. Such a condition, though difficult to realize in a laboratory experiment, does, however, present an interesting scenario. Such a boundary condition could be generated by placing stiffeners with infinite inplane bending stiffness and zero axial stiffness along the simply supported edges of the plate. Such stiffeners would be able to ensure that the $v$ displacement is a constant along the simply supported edge. In reality, the placement of stiffeners along the simply supported edges would not only restrict the variation of $v$ displacement along the simply supported edge, but would also increase the axial load borne by the plate. In this regard, it would be important to investigate the relationship between the boundary conditions, stiffener thickness, and secondary buckling. This needs to be done, especially for clamped plates with aspect ratios close to unity, since they are quite frequently used in structures.

2. The question as to how imperfections influence secondary buckling is also an unresolved one. According to Stoll [25], an imperfection in the shape of primary symmetric buckling mode could slightly increase the secondary buckling load. However, any imperfection in the shape of an anti-symmetric buckling mode could significantly reduce the secondary buckling load. Stoll's observations are valid for plates with an aspect ratio of 5.38. However, according to Shin [24], imperfections could lead to a delay in the mode change for isotropic plates with similar aspect ratios. Issues like this one need to be resolved. There are also issues involving 
the relative influence of magnitudes of geometric imperfections vis a vis global material imperfections, such as a misaligned ply. Another class of imperfections which need to be studied are local in nature. These could be due to processing defects, material defects, or impact damage. In these cases, material properties could have a nonzero gradient in the thickness direction of the plate.

3. So far, secondary buckling in composite plates has not been investigated in great detail. The work in clamped plates made of composite materials is even less. Further, investigators have usually neglected the influence of bending-twisting coupling coefficients on account of their smallness. However, in view of the probable imperfection sensitivity of panels to the secondary buckling loads $[24,25]$, the roles played by $D_{16}$ and $D_{26}$ merits attention.

4. Most of the investigators have employed series expressions with a view to explain the phenomenon of secondary buckling. The issue concerning the number of terms to be used in these expressions which shall ensure convergence of displacement variables has been a contentious one. Further, such an approach is mathematically quite intensive and tedious. Still further, diverse effects, such as those due to imperfections, boundary conditions, stiffener properties, and the roles of $D_{16}$ and $D_{26}$, cannot be easily accounted for by such a solution strategy. In, view of all these constraints, a numerical approach like the finite element method is perhaps more suitable and efficient way of approaching the problem. This is despite the fact that it is comparatively more difficult to conduct parametric studies with the finite element method. The approach of Newton-Raphson can be employed to find the solution to simultaneous nonlinear algebraic equations. The stability of the system could be found by checking the sign of eigenvalues of the tangent stiffness matrix. By using eigenvectors associated with a near-zero positive eigenvalue as perturbations, transition from one branch of the solution to another one could be made. Details of such a branching strategy have been proposed by Thurston [29].

\subsection{A ROAD MAP TO THE REMAINING CHAPTERS}

In view of the observations made in section 1.3, for this current research a series of numerical and experimental investigations were conducted to attain deeper understanding of secondary buckling 
in almost-square plates. The following chapters are a report of the methodologies employed, the results as a consequence of these investigations, and the overall conclusions arrived at.

Chapter 2 enlists the details of the approach used to analyze the phenomenon of secondary buckling. The finite element method was used to analyze the plates from a numerical point of view. Consequently, a FORTRAN code was developed to conduct finite element investigations for twodimensional thin anisotropic laminated plates subjected to inplane and out-of-plane loads. The code was developed with a view to incorporate diverse features, such as geometric nonlinearity dues to moderate rotations, the role of out-of-plane geometric imperfections, locally different material properties, bending-stretching coupling, and the effects of shear deformations. The code was able to provide displacement, strain, and stress fields in the deformed plate, and also could conduct stability and bifurcation analyses. Chapter 2 provides the details of various theories employed for purposes of achieving the ends as outlined above. Finally, this chapter also provides the details of various case studies which were used as benchmarks for purposes of validating the code.

Chapter 3 details the results of various finite element studies of plates having an aspect ratio close to unity subjected to uniform inplane end shortening. These plates were clamped along the loading edges, and simply supported along the remaining two edges. Thus both the out-of-plane displacement as well as the out-of-plane rotations were constrained to be zero along the clamped edges, while only the out-of-plane displacement was specified as zero along the simply supported edges. This clamped condition is representative of the conditions used in experiments.

The inplane boundary conditions were varied for these plates. This variation of the boundary conditions was done for edges 2 and 4 as identified in Fig. 1.1-a. The first set of inplane boundary conditions required the inplane normal displacement to be zero along edges 2 and 4 . The second set of inplane boundary conditions imposed no restraint on inplane normal displacement, thereby implying that the external stress resultants along edges 2 and 4 was zero. The third set of inplane boundary conditions specified inplane normal displacement as a uniform but unknown constant along edges 2 and 4, and also that the net external force in $y$ direction along these edges was zero. This third set of boundary conditions is purely an academic construct, since the simulation of 
these type of boundary conditions in laboratory is extremely difficult. However, it was felt that these boundary conditions can be duplicated in the laboratory in a limited sense by placing stiffeners along the simply supported edges 2 and 4. It was thought that the inplane bending stiffness of the stiffeners would substantially constrain the variation of inplane normal displacement along edges 2 and 4, thereby bringing it closer to a constant. Thus finite element studies for stiffened plates were also conducted. A series of these studies were conducted by varying the thickness and width of the stiffeners. Also, parametric studies were conducted to explore the sensitivity of secondary buckling response of these plates to changes in lamination sequence, presence of imperfections, and magnitude of out-of-plane shear deformations. Finally, the response of these plates was compared to that of metallic plates. Details of these studies have been provided in Chapter 3.

As mentioned earlier, experimental studies were also conducted. These were done for plates with clamped conditions along the loading edges 1 and 3, as shown in Fig. 1.1-a, and simple supports along the remaining two edges 2 and 4. Both stiffened as well as unstiffened plates with various lamination sequences were tested. Chapter 4 provides the details of the associated experimental fixture, the loading frame, and the accompanying instrumentation and data acquisition systems. Chapter 5 provides a comparison of experimental results and the finite element predictions. Chapter 6 is conclusive in nature. It lists the key findings of this research, and also recommends the direction of future work to be conducted to further the understanding of secondary buckling in composite plates. 


\section{FINITE ELEMENT FORMULATION AND VALIDATION}

\subsection{NONLINEAR SHEAR DEFORMABLE THEORY}

The plates studied are analyzed by a theory based on an amalgam of the plate theories of von Karman and Mindlin. While the former theory works well when nonlinearities because of moderate rotations are present, the latter theory by Mindlin was used to capture the effects of shear deformations, if any, on the behavior of the plates. However, here the Mindlin approach was developed not so much because of the expectation of significant shear deformation effects, but rather because it results in lower-order governing differential equations compared to classical Kirchhoff theory. As a consequence, finite element formulation of Mindlin-Reissner plate theory allows for lower-order displacement field approximations than Kirchhoff theory. To account for the fact that in practice most plates are not perfectly flat, Marguerre's theory of accounting for an initial outof-plane deflection has been used. Details of this theory can be found in [30].

\subsubsection{Geometry}

Figure 2.1 shows the location and the orientation of the reference coordinate system with respect to the plate geometry. Specific boundary conditions are shown, with ss and c denoting simply supported and clamped, respectively, as regards out-of-plane displacements. The direction of inplane loading and the transverse inplane direction have been designated as $x$ and $y$, respectively. The position vectors in the undeformed and the deformed geometries are $\bar{r}_{o}$ and $\bar{r}$, respectively. 


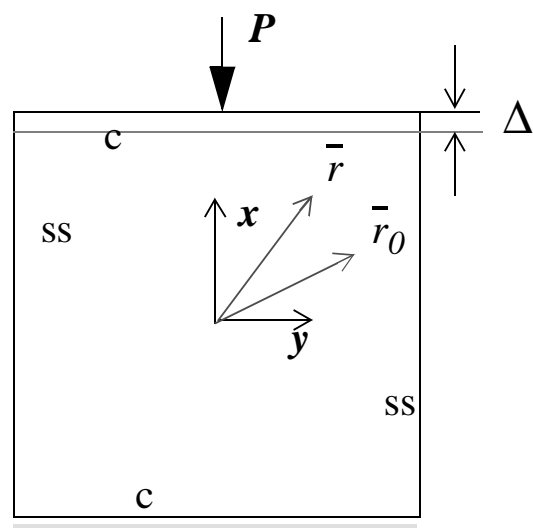

Fig. 2.1: Plate Under Uniform Compression

The position vectors $\bar{r}$ and $\bar{r}_{o}$ can be expressed as

$$
\begin{gathered}
\bar{r}_{0}(x, y, z)=x \hat{\imath}+y \hat{\jmath}+(z+\xi(x, y)) \hat{k} \\
\bar{r}(x, y, z)=(x+u(x, y, z)) \hat{\imath}+(y+v(x, y, z)) \hat{\jmath}+(z+(\xi(x, y)+w(x, y, z))) \hat{k},
\end{gathered}
$$

where, $u, v, w$ are the displacements along the $x, y$, and $z$ directions, and $\xi$ the function representing out-of-plane imperfections. The displacement field is of the following form:

$$
\left[\begin{array}{l}
u(x, y, z) \\
v(x, y, z) \\
w(x, y, z)
\end{array}\right]=\left[\begin{array}{c}
u^{o}(x, y) \\
v^{o}(x, y) \\
w^{o}(x, y)
\end{array}\right]+z\left[\begin{array}{c}
\psi_{x}^{o}(x, y) \\
\psi_{y}^{o}(x, y) \\
0
\end{array}\right]
$$

where, $\psi^{0}{ }_{x}$ and $\psi_{y}^{0}$ are the rotations of the midplane of the plate in the $x$ - $z$ and $y-z$ planes, respectively, and $u^{o}, v^{o}$, and $w^{o}$ are the displacements of the midplane of the plate.

\subsubsection{Strain-Displacement Relations}

The shortest distances between two points lying within the plate in the undeformed and the deformed geometries can be written as $s_{o}$ and $s$, respectively. The differentials of these metrics 
can be written as

$$
d s_{o}^{2}=\overline{d r}_{O} \cdot \overline{d r}_{o} \quad d s^{2}=\overline{d r} \cdot d \bar{r}
$$

It is also known from the principles of geometry that

$$
d s^{2}-d s_{o}^{2}=2\left[\varepsilon_{x x} d x^{2}+\varepsilon_{y y} d y^{2}+\varepsilon_{z z} d z^{2}+\gamma_{x y} d x d y+\gamma_{y z} d y d z+\gamma_{z x} d z d x\right]
$$

The strain-displacement relations used in the present study can be extracted from Eqs. (3) and (4). These relations can be written as

$$
\begin{gathered}
\varepsilon_{x x}=\frac{\partial u^{o}}{\partial x}+\frac{1}{2}\left(\frac{\partial w^{o}}{\partial x}\right)^{2}+z\left(\frac{\partial \psi}{\partial x}\right)+\frac{\partial \xi \partial w^{o}}{\partial x \partial x}=\varepsilon_{x}^{o}+z \kappa_{x}^{o} \\
\varepsilon_{y y}=\frac{\partial v^{o}}{\partial y}+\frac{1}{2}\left(\frac{\partial w^{o}}{\partial y}\right)^{2}+\frac{\partial \psi}{\partial y}+\frac{\partial \xi \partial w^{o}}{\partial y \partial y}=\varepsilon_{y}^{o}+z \kappa_{y}^{o} \\
\gamma_{x y}=\frac{\partial u^{o}}{\partial y}+\frac{\partial v^{o}}{\partial x}+\frac{\partial w^{o}}{\partial x} \frac{\partial w^{o}}{\partial y}+z\left(\frac{\partial \psi}{\partial y}+\frac{\partial \psi}{\partial x}\right)+\left(\frac{\partial \xi \partial w^{o}}{\partial x \partial y}+\frac{\partial w^{o}}{\partial x} \frac{\partial \xi}{\partial y}\right)=\Upsilon_{x y}^{o}+z \kappa_{x y}^{o} \\
\gamma_{z x}^{0}=\psi_{x}^{o}+\frac{\partial w^{o}}{\partial x} \\
\gamma_{y z}^{0}=\psi_{y}^{o}+\frac{\partial w^{o}}{\partial y}
\end{gathered}
$$

Here, $\varepsilon_{x x}$ and $\varepsilon_{y y}$ are the normal strains in the plate in $x$ and $y$ directions, respectively, while $\gamma_{x y}$ is the shear strain in the plane of the plate. Further, these strains have been resolved into their respective midplane and bending components. The midplane components of strains $\varepsilon_{x x}$, $\varepsilon_{y y}$, and $\gamma_{x y}$ have been designated as $\varepsilon_{x x}^{0}, \varepsilon^{0}{ }_{y y}$, and $\gamma_{x y}^{0}$, respectively. The bending components have been expressed in terms of their respective curvatures, $\kappa^{0}{ }_{x x}, \kappa^{\mathrm{O}}{ }_{y y}$, and $\kappa^{\mathrm{O}}{ }_{x y}$, and the distance from the midplane, $z$. Finally, the out-of-plane shear strains, $\gamma_{y z}^{0}$ and $\gamma_{z x}^{0}$, have been also defined in Eqs. (5). In arriving at these relations, terms involving higher powers of $\partial \xi / \partial x, \partial \xi / \partial y, \partial u / \partial x, \partial v / \partial y$, 
etc., have been neglected on account of their smallness compared to other terms.

\subsubsection{Variational Statement}

For the problem at hand, the first variation of the total potential energy, $\delta \Pi$, can be written as

$$
\delta \Pi=\int_{\Omega}\left[\sigma_{x x} \delta \varepsilon_{x x}+\sigma_{y y} \delta \varepsilon_{y y}+\tau_{x y} \delta \gamma_{x y}+\tau_{y z} \delta \gamma_{y z}+\tau_{z x} \delta \gamma_{z x}\right] d \Omega-\int_{A} f \delta w d A
$$

Here, $\Omega$ and $A$ represent the volume and planform surface area of the plate, respectively. Variational principles dictate that for the static equilibrium of such a plate, the above variation has to be zero. It must be noted here that the total potential energy is an algebraic sum of the internal strain energy density integrated over the volume, and the external work done. The external work done is a consequence of external loading, $f$ applied over the normal area $A$. While the external load $f$ is not used in actual problem as described in section 2.1, it has been considered in the finite element formulation since some of the validation problems do involve it. Equation (6) neglects the contribution of $\delta \varepsilon_{z z}$ to the overall variation in total potential energy. This is based on the assumption that the $w$ displacement does not vary in the $z$ direction, and hence the strain $\varepsilon_{z z}$ as well as its variation in the $z$ direction are identically zero.

Substituting Eqs. (5) in Eq. (6) and integrating the resulting equation in the thickness direction, the following equation is achieved:

$$
\begin{aligned}
\delta \Pi= & \int_{A}\left[N_{x} \delta \varepsilon^{o}{ }_{x}+N_{y} \delta \varepsilon^{o}{ }_{y}+N_{x y} \delta \gamma_{x y}^{o}+Q_{1} \delta \gamma_{x z}^{o}+Q_{2} \delta \gamma_{y z}^{o}\right] d A- \\
& \int_{A}\left[M_{x} \delta \kappa^{o}{ }_{x}+M_{y} \delta \kappa^{o}{ }_{y}+M_{x y} \delta \kappa^{o}{ }_{x y}\right] d A-\int_{A} f \delta w d A=0
\end{aligned}
$$


Here, $N \mathrm{~s}, Q \mathrm{~s}$, and $M \mathrm{~s}$ are force and moment resultants which are defined as

$$
\begin{gathered}
\left(N_{x}, N_{y}, N_{x y}\right)=\int_{-h / 2}^{h / 2}\left(\sigma_{x x}, \sigma_{y y}, \tau_{x y}\right) d z \quad\left(M_{x}, M_{y}, M_{x y}\right)=\int_{-h / 2}^{h / 2}\left(\sigma_{x x}, \sigma_{y y}, \tau_{x y}\right) z d z \\
\left(Q_{x}, Q_{y}\right)=\int_{-h / 2}^{h / 2}\left(\tau_{y z}, \tau_{z x}\right) d z
\end{gathered}
$$

where $h$ is the thickness of the plate. It must be noted that while $N_{x}, N_{y}$, and $N_{x y}$ are inplane force resultants, $Q_{x}$, and $Q_{y}$ are out-of-plane shear force resultants.

Incorporating the definitions of midplane strains and curvatures from Eqs. (5) in Eq. (7), followed by integrating the resulting equation by parts and rearranging the terms by collecting the terms associated with $\delta u^{o}, \delta v^{o}, \delta w^{o}, \delta \psi^{o}{ }_{x}$ and $\delta \psi^{o}{ }_{y}$, yields five equations of equilibrium. These are:

$$
\begin{aligned}
& \frac{\partial N}{\partial x}^{x}+\frac{\partial N}{\partial y}^{x y}=0 ; \quad \frac{\partial N}{\partial x}^{x y}+\frac{\partial N}{\partial y}^{y}=0 \\
& \frac{\partial w}{\partial x}\left(\frac{\partial N_{x}}{\partial x}+\frac{\partial N_{x}}{\partial y} y\right)+\frac{\partial w}{\partial y}\left(\frac{\partial N_{x y}}{\partial x}+\frac{\partial N_{y}}{\partial y}\right)=\frac{\partial Q_{1}}{\partial x}+\frac{\partial Q_{2}}{\partial y}-f(x, y) \\
& \frac{\partial M}{\partial x}^{x}+\frac{\partial M_{x y}}{\partial y}=Q_{x} ; \quad \bar{\partial}_{x y}{ }^{+\frac{\partial M}{\partial y}}{ }^{y}=Q_{y} .
\end{aligned}
$$

\subsubsection{Constitutive Laws}

Assuming the material to be linearly elastic, the constitutive relations for an anisotropic material can be written in the following form 


$$
\begin{gathered}
{\left[\begin{array}{c}
N_{x} \\
N_{y} \\
N_{x y} \\
M_{x} \\
M_{y} \\
M_{x y}
\end{array}\right]=\left[\begin{array}{llllll}
A_{11} & A_{12} & A_{16} & B_{11} & B_{12} & B_{16} \\
A_{12} & A_{22} & A_{26} & B_{21} & B_{22} & B_{26} \\
A_{16} & A_{26} & A_{66} & B_{16} & B_{26} & B_{66} \\
B_{11} & B_{21} & B_{61} & D_{11} & D_{12} & D_{16} \\
B_{12} & B_{22} & B_{62} & D_{12} & D_{22} & D_{26} \\
B_{16} & B_{26} & B_{66} & D_{16} & D_{26} & D_{66}
\end{array}\right]\left[\begin{array}{c}
\varepsilon_{x x}^{0} \\
0 \\
\varepsilon_{y y} \\
0 \\
\gamma_{x y} \\
\kappa_{x x} \\
\kappa_{y y} \\
\kappa_{x y}^{0} \\
\kappa_{x y}
\end{array}\right]-\left[\begin{array}{c}
N_{x}^{T} \\
N_{y}^{T} \\
N_{x y}^{T} \\
M_{x}^{T} \\
M_{x}^{T} \\
M_{x y}^{T}
\end{array}\right] \Delta T} \\
{\left[\begin{array}{l}
Q_{x} \\
Q_{y}
\end{array}\right]=\left[\begin{array}{lll}
A_{44} & A_{45} \\
A_{45} & A_{55}
\end{array}\right]\left[\begin{array}{c}
o \\
\gamma_{x y}^{o} \\
\gamma_{z x}^{o}
\end{array}\right]}
\end{gathered}
$$

Here, quantities $A_{i j}, B_{i j}$, and $D_{i j}$ are members of extension, extension-bending coupling, and bending stiffness matrices. Definitions of these matrices can be found in Ref. [26]. Further, terms $N^{T}{ }_{x}$ $N_{y}^{T}, N_{x y}^{T}$ and $M_{x}^{T}, M_{y}^{T}, M_{x y}^{T}$ are thermal force and moment resultants, and $\Delta T$ is the change in temperature of the laminate material. Their definitions can also be found in Ref. [26].

\subsection{FINITE ELEMENT FORMULATION}

Equation (9) can be used for the finite element formulation by conducting the following steps:

- Multiply Eqs. (9a-e) by $\delta u^{o}, \delta v^{o}, \delta w^{o}, \delta \psi^{o}{ }_{x}$ and $\delta \psi^{o}{ }_{y}$ respectively.

- Substitute Eqs. (10) and Eqs. (5) in the equations obtained from the first step.

- Integrate the resulting equations by parts over the finite two-dimensional area of the plate's reference surface, the midplane of the plate

- Approximate the variations in displacements $\left(\delta u^{o}, \delta v^{o}, \delta w^{o}, \delta \psi_{x}^{o}\right.$, and $\left.\delta \psi_{y}^{o}\right)$ and displacements $\left(u^{o}, v^{o}, w^{o}, \psi_{x}^{o}\right.$, and $\left.\psi^{o}{ }_{y}\right)$ by combinations of known Lagrangian shape functions, $\phi$, and unknown nodal constants, $c$. An isoparametric formulation was used. Here, it was assumed that the shape functions associated with displacements and its variations 
were the same. Here, the shape functions were specific to nine-noded isoparametric elements. Each node of the finite element was assigned five degrees of freedom. The Gaussian integration scheme was used to compute the terms in the ensuing stiffness matrix of the finite element. Finally, reduced integration method was used to resolve the issue of shear-locking of the plate.

Using such a methodology, the following nonlinear finite element equations were obtained:

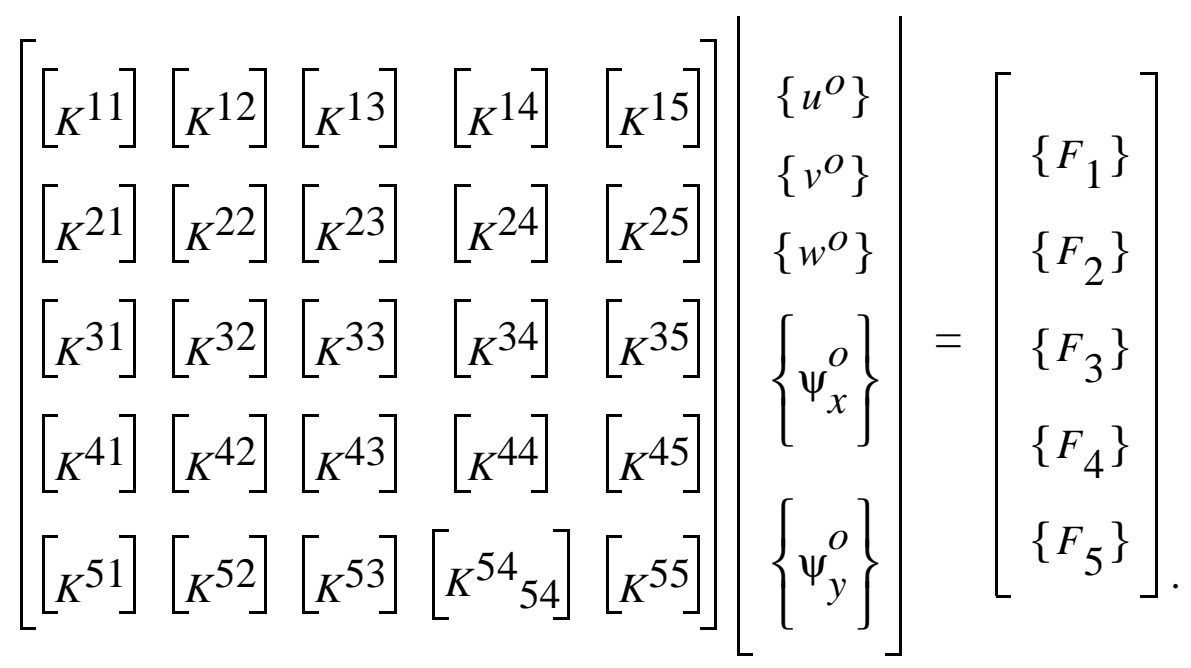

Equation (11) is a statement of equilibrium in the three translational directions $(x, y, z)$ and two rotational directions lying in the $x-z$ and $y-z$ planes. Fully developed expressions of the terms in tangent stiffness matrix have been given in Appendix A. These equations are nonlinear in nature, since the sub-matrices $\left[K^{i j}\right]$ are functions of the displacement field $\left(u^{o}, v^{o}, w^{o}, \psi^{o}{ }_{x}\right.$, and $\left.\psi_{y}^{o}\right)$. Subsequently, the iterative method of Newton-Raphson was used to solve these equations. 
Employing such an approach leads to incremental equations of equilibrium, which are

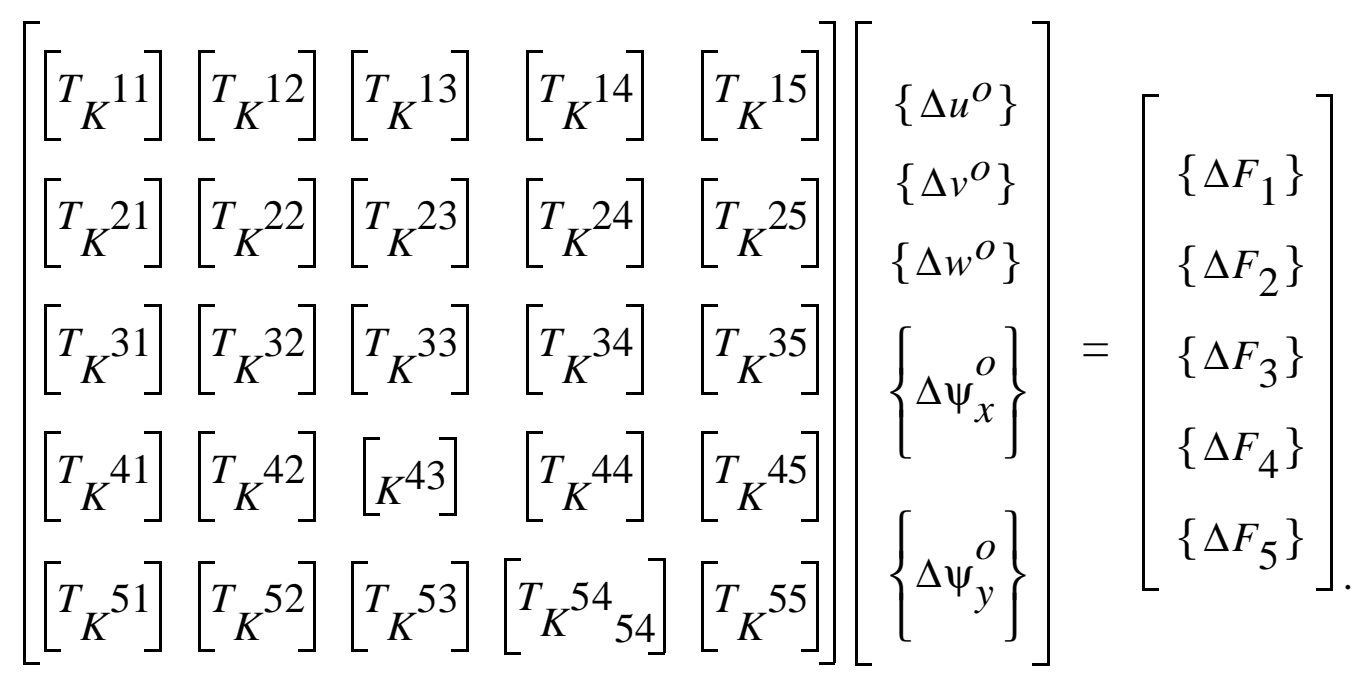

Here, submatrices $\left[{ }^{T} K^{i j}\right]$ are tangent stiffness sub-matrices, which are functions of the displacement field $\left(u^{o}, v^{o}, w^{o}, \psi_{x}^{o}\right.$, and $\left.\psi_{y}^{o}\right)$. The term tangent stiffness has been used since in a onedimensional problem the associated matrix $\left[{ }^{T} K\right]$ represents the slope of the tangent of the solution path.

The displacement field has been computed in an incremental fashion. So, instead of computing $u^{o}$, $v^{o}, w^{o}, \psi_{x}^{o}$, and $\psi_{y}^{o}$, their increments $\Delta u^{o}, \Delta v^{o}, \Delta w^{o}, \Delta \psi^{o}{ }_{x}$, and $\Delta \psi_{y}^{o}{ }_{y}$ were computed in an iterative fashion. The right hand side of Eqs. (12) represents the residual force vector, the magnitude of which, at the point of convergence, is quite small. Details of this approach can be found in Ref. [31]. It must be noted that Eqs. (11) and (12) are at the element level. These equations were later assembled for the entire physical system. Details of this process are given in Ref. [32]. Boundary conditions were subsequently imposed on the ensuing assembled set of equations, thereby reducing their number. Finally, the consequential reduced set of equations were solved for incremental unknown displacement field with the help of Gaussian elimination procedure using IMSL subroutines [33].

It may be noted that while the stiffness matrix in Eq. (11) lacks symmetry, the tangent stiffness matrix $\left[{ }^{T} K\right]$ is banded as well as symmetric. This feature of the tangent stiffness matrix is benefi- 
cial, since it leads to a saving of work space on computers.

\subsubsection{Imposition of Constraint Equations}

One of the boundary conditions considered during the analysis involved equations that constrain a particular set of nodal displacement variables to remain mutually equal. Specifically, referring to Fig. 2.1, the simply supported side edges were assumed to move in a uniform fashion in the $y$ direction in some analyses considered in the present study. Imposition of these constraints was carried out using the following approach. Let the assembled set of incremental variables be represented as

$$
\left[\begin{array}{ccc}
{[A]} & {[B]} & {[C]} \\
{[D]} & {[E]} & {[F]} \\
{[G]} & {[H]} & {[I]}
\end{array}\right]\left[\begin{array}{l}
\left\{X^{1}\right\} \\
\left\{X^{2}\right\} \\
\left\{X^{3}\right\}
\end{array}\right]=\left[\begin{array}{l}
\left\{f^{1}\right\} \\
\left\{f^{2}\right\} \\
\left\{f^{3}\right\}
\end{array}\right] .
$$

Here $\{X\}$ is the global solution vector, which has been partitioned into three subvectors, $\left\{X^{1}\right\}$, $\left\{X^{2}\right\}$, and $\left\{X^{3}\right\}$, having lengths of $k, l$, and $m$, respectively. The overall length of vector $\{X\}$ is $n$. Consequentially it follows that

$$
k+l+m=n
$$

Since Eqs. (13) are $n$ in number, and involve $n$ variables, it can be inferred that the dimensions of

\begin{tabular}{|c|c|c|}
\hline$[A] \quad(k X k)$ & {$[B] \quad(k X l)$} & {$[C] \quad(k X m)$} \\
\hline [D] $(l X k)$ & {$[E] \quad(l X l)$} & {$[F] \quad(l X m)$} \\
\hline$G] \quad(m X k)$ & {$[H] \quad(m X l)$} & $(m X m)$. \\
\hline
\end{tabular}
submatrices listed in Eqs. (13) are as follows:

Also, if $\left\{X^{1}\right\}$ represents $k$ independent unknown primary variables, $\left\{X^{2}\right\}$ constitutes $l$ known primary variables, and $\left\{X^{3}\right\}$ represents $m$ unknown variables constrained as

$$
X_{s}^{3}=X_{1}^{3} \quad s=1, m
$$


then the total number of independent unknown variables is $(n-l-m+1)$. The aim here is to reduce Eq. (13), which consists of $n$ in number, to another set of equations, which are $(n-l-m+1)$ in number. This is achieved in two steps by

- Eliminating (m-1) equations by imposing the constraints of Eq. (15).

- Eliminating $l$ equations by imposing the known primary variables. This step is conducted only after the prior step has been achieved. Details of this process can be found in [32].

The imposition of constraint conditions on Eq. (13) has been achieved by conducting the following mathematical operations. From Eq. (13), the first set of equations can be rewritten as

$$
\sum_{j=1}^{k} A_{i j} X_{j}^{1}+\sum_{j=1}^{l} B_{i j} X_{j}^{2}+\sum_{j=1}^{m} C_{i j} X_{j}^{3}=f_{i}^{1} \quad i=1, k
$$

Imposing Eq. (15) on this set of equations, Eq. (16) can be rewritten as

$$
\begin{gathered}
\sum_{j=1}^{k} A_{i j} X_{j}^{1}+\sum_{j=1}^{l} B_{i j} X_{j}^{2}+\bar{C}_{i 1} X_{1}^{3}=f_{i}^{1} \quad i=1, k \\
\text { where, } \bar{C}_{i 1}=\sum_{j=1}^{m} C_{i j} .
\end{gathered}
$$

Using such an approach, the following set of $(n-m+1)$ equations is written

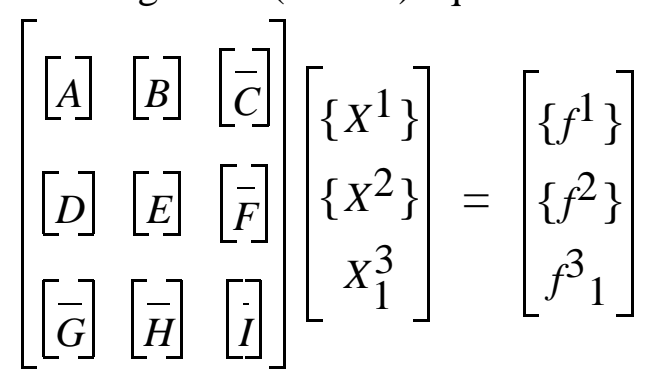


where the elements of newly constructed matrices $[\bar{C}],[\bar{F}],[\bar{G}],[\bar{H}]$, and $[\bar{I}]$ are defined as

$$
\begin{array}{ccc}
\bar{C}_{i 1}=\sum_{\substack{j=1 \\
m}}^{m} C_{i j} & \bar{F}_{i 1}=\sum_{j=1}^{m} F_{i j} & \bar{G}_{1 i}=\sum_{j=1}^{m} G_{j i} \\
\bar{H}_{1 i}=\sum_{j=1}^{m} H_{j i} & \bar{I}_{11}=\sum_{i=1 j=1}^{m} \sum_{i j}^{m} I_{i j} & f_{1}^{3}=\sum_{j=1}^{m} f_{j}^{3} .
\end{array}
$$

It must be noted that the forcing term $f_{1}^{3}$ is a known boundary condition. It must also be stated that this approach of imposing the constraint conditions does not destroy the symmetry of the tangent stiffness matrix.

\subsection{STABILITY CONSIDERATIONS AND BIFURCATION ANALYSIS}

As explained earlier, the employment of Newton-Raphson's method for solving Eq. (11) after the assembly leads to a set of equations which are incremental in nature and can be written as

$$
\left[K^{T}(U)\right]\{\Delta U\}=\{\Delta F\},
$$

where $\left[K^{T}(U)\right]$ refers to the tangential stiffness matrix, $\{\Delta U\}$ is the incremental solution vector, and $\{\Delta F\}$ is the residual force vector. Since Eq. (20) is nonlinear in nature, it can have more than one solution for a given set of boundary conditions. Some of these solutions, when plotted in a load versus end shortening space, can intersect each other. These points of intersections are called bifurcation points. From a mathematical standpoint, all of these solutions are equally valid and equally possible. However, the physical system under study prefers to chose a configuration which is stable in the sense that the potential energy $\Pi$ of the system is at a relative minimum in the neighborhood of the position of equilibrium. This can be mathematically expressed as

$$
\left.\delta^{2} \Pi\right|_{\delta \Pi=0}>0 .
$$


Equation (21) is the statement of stability of an equilibrium solution for conservative systems. Using this as a condition for stability, it can been shown that a solution for Eq. (20) is stable only if the quantity $\{\delta \Delta U\}^{T}\left[K^{T}(U)\right]\{\delta \Delta U\}$ is positive for any given set of physically admissible variations of the incremental solution vector, represented by $\{\delta \Delta U\}$. Further, it can be shown mathematically that the quantity $\{\delta \Delta U\}^{T}\left[K^{T}(U)\right]\{\delta \Delta U\}$ is positive definite if all the eigenvalues of the tangent stiffness matrix are positive. This criterion was employed in the analysis to check the stability of the plate at each load step. To economize on the computational costs, only the lowest five eigenvalues were examined at each load step. Extraction of eigenvalues and the associated eigenvectors was carried out with the help of IMSL subroutines [33].

As for the analysis of bifurcation points, consider the hypothetical load versus end shortening relation for an initially flat plate, such as shown in Fig. 2.2

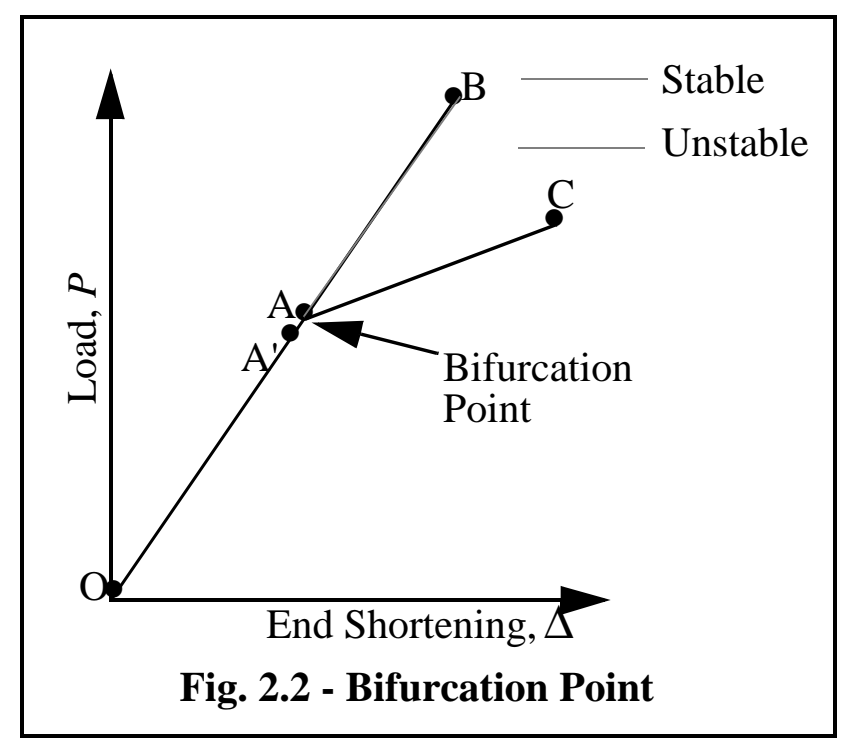

Here path O-A-B represents solutions for which the plate remains flat. Point $\mathrm{A}^{\prime}$ is on path OA, just a small distance before A. Segment OA is the stable part of this solution path. If the plate is loaded beyond point $\mathrm{A}$ on to the path $\mathrm{O}-\mathrm{A}-\mathrm{B}$, it becomes unstable. Line AC represents an alternative solution path which is stable. The instability at A can be detected by checking the eigenvalues of the tangent stiffness matrix. A negative eigenvalue is the signal for instability, while a positive eigenvalue indicates that the associated plate configuration is a stable one. Further, if the eigenvalue is positive, but very close to zero, it indicates that the existing solution path is very soon 
going to become unstable. The move to path AC can be accomplished numerically by using Thurston's approach [29] which can be summarized as follows:

- Starting from point $\mathrm{O}$, approach point $\mathrm{A}^{\prime}$. The solution path O-A'-A-B is stable up to point A. Point A' being close to point A implies that at point $\mathrm{A}^{\prime}$ the eigenvalues of the tangent stiffness matrix are positive, but also that at least one of these eigenvalues is very close to zero. Point A', in essence, is on the verge of instability. Let the solution vector at this point be $\left\{U_{\mathrm{A}^{\prime}}\right\}$ and the associated end shortening be $\Delta_{\mathrm{A}^{\prime}}$.

- Compute the normalized eigenvector at point $\mathrm{A}^{\prime}$ associated with the eigenvalue approaching zero. Let this vector be $\{E\}$. The vector is normalized in the sense of the following equation:

$$
\left(\sum_{i=1}^{n} E_{i} E_{i}\right)^{1 / 2}=1 .
$$

- Using $\left(\left\{U_{\mathrm{A}^{\prime}}\right\}+\lambda\{E\}\right)$ as the guess solution vector for the load step corresponding to increased end shortening, Eq. (20) can be solved. Such an approach helps in switching from path O-A-B to path O-A-C in the neighborhood of point A. Here, $\lambda$ is a scalar parameter which has to be determined by a trial and error approach. For certain situations, even a negligibly small value of $\lambda$ is sufficient to move the system from path $\mathrm{O}$ A-B to O-A-C. One simple example of such a case is when a plate subjected to an inplane compressive displacement moves from a flat configuration to a buckled configuration. However, when the plate is deep into the post-buckling range, a relatively higher value of $\lambda$ has to be used to move it from a primary buckling configuration to a secondary buckling configuration.

\subsection{VALIDATION OF THE FINITE ELEMENT CODE}

This section focuses on the robustness of the finite element code which was developed for the 
present research. To check the validity of the code, several benchmarks have been used. The validation of the code was done in a systematic way, so that the role played by all the elements of the $A, B$, and $D$ stiffness matrices stood individually verified and tested. What follows is a detailed summary of these benchmark comparisons.

\subsubsection{Unsymmetrically Laminated Linear Rectangular Composite Plate Under Uniform End Compression}

This benchmark was designed to check the geometrically linear capabilities of the code. The choice of the lamination sequence was also guided by the objective to have fully populated $A, B$, and $D$ matrices. The details of the problem are given in Fig. 2.3.

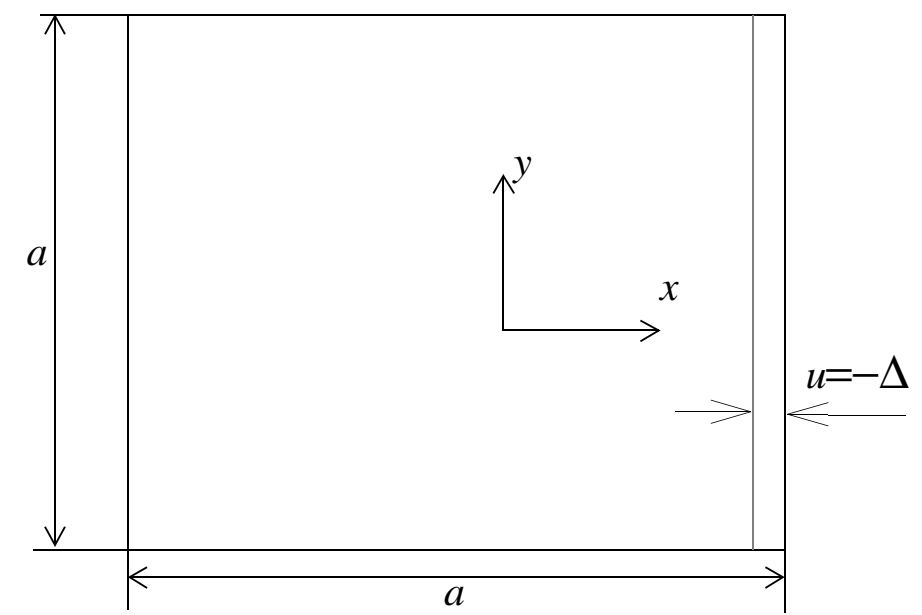

Fig. 2.3: Unsymmetrically Laminated Linear Plate Under Uniform End Compression
Material Properties:

$E_{11}=127.9 \mathrm{GPa}$

$E_{22}=11.06 \mathrm{GPa}$

$\mathrm{G}_{12}=5.750 \mathrm{GPa}$

$v_{12}=0.35$

Layup: [0/-45]

Thickness of each layer $=1 \mathrm{~mm}$. $a=0.25 \mathrm{~m}$.

Boundary Conditions:

$u=0$ at $x=-a / 2, u=-\Delta$ at $x=a / 2$

$v=w=0$ at $(x, y)=(-a / 2,-a / 2)$

Mesh: 5 X 5 grid of 9 noded Lagrangian quadratic elements.

Such a plate has uniform strain and curvature fields. The value of strain in $x$ direction is $-\Delta a$. It can be shown with the help of classical theory for laminated plates that the strains and curvatures 
of the plate as defined in Fig. 2.3 can be written as:

$$
\left[\begin{array}{c}
\varepsilon_{y y}^{o} \\
\gamma_{x y}^{o} \\
\kappa_{x x}^{o} \\
\kappa_{y y}^{o} \\
\kappa_{x y}^{o}
\end{array}\right]=\left[\begin{array}{lllll}
A_{22} & A_{26} & B_{12} & B_{16} & B_{26} \\
A_{26} & A_{66} & B_{16} & B_{26} & B_{66} \\
B_{12} & B_{16} & D_{11} & D_{12} & D_{16} \\
B_{22} & B_{26} & D_{12} & D_{22} & D_{26} \\
B_{26} & B_{66} & D_{16} & D_{26} & D_{66}
\end{array}\right]^{-1}\left[\begin{array}{l}
-A_{12} \\
-A_{16} \\
-B_{11} \\
-B_{12} \\
-B_{16}
\end{array}\right] \varepsilon_{x x}^{o}
$$

The following table compares the results obtained from the above equations with those obtained from the finite element code developed.

Table 2.1: Comparison of FEM and Exact Results for a Unsymmetrically Laminated Linear Plate

\begin{tabular}{|l|l|l|l|l|l|}
\hline & \multicolumn{1}{|c|}{$\varepsilon^{o}{ }_{y y} / \varepsilon^{o}{ }_{x x}$} & $\gamma_{x y}^{o} / \varepsilon_{x x}^{o}$ & $\kappa^{0}{ }_{x x} / \varepsilon_{x x}^{o}$ & $\kappa^{o}{ }_{y y} / \varepsilon_{x x}^{o}$ & $\kappa_{x y}^{0} / \varepsilon_{x x}^{o}$ \\
\hline \hline Classical Theory & -0.3339 & 0.52841 & 1183.9 & -353.08 & 830.8 \\
\hline FEM & -0.3339 & 0.52842 & 1183.7 & -353.08 & 830.6 \\
\hline
\end{tabular}

\subsubsection{Isotropic Plates Subjected to Uniform Lateral Pressure}

These cases test the program's ability to predict the nonlinear behavior of isotropic plates. Three cases are discussed.

\subsubsection{Simply Supported Isotropic Square Plate}

Here a square, isotropic plate which is simply supported on all four sides and subjected to a uniform pressure loading $q_{o}$ is considered. There are no restraints on inplane motion along the edges. The boundary conditions and the material properties are given Fig. 2.4. The problem considered is 
mildly nonlinear, as the out-of-plane rotations of the plate are developed enough to generate a nonlinear response. Figure 2.5 is a comparison of the FEM results with the analytical series solution developed by Chia [30].

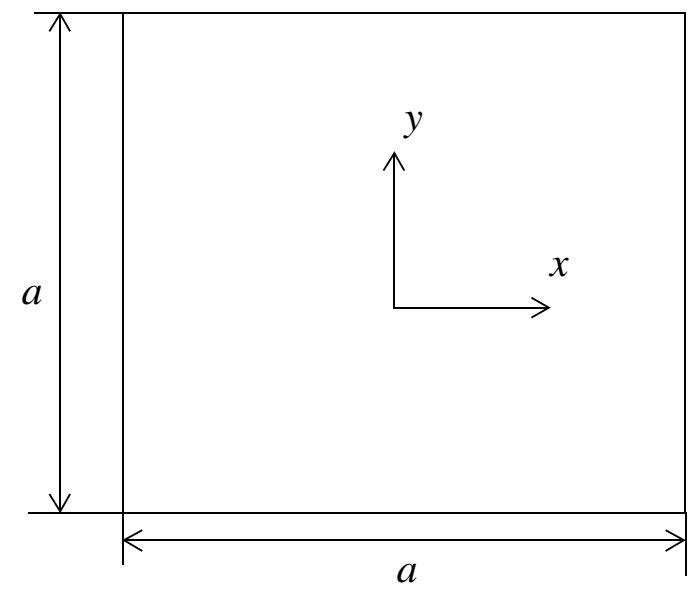

Young's Modulus $(E)=200.0 \mathrm{GPa}$

Poisson's ratio $(\mathrm{V})=0.316$

Plate thickness(h): $2 \mathrm{~mm}$.

Boundary Conditions:

$u=0$ at $x=-a / 2, v=0$ at $(x, y)=(-a / 2,-a / 2)$ $w=0$ at $x= \pm a / 2, y= \pm a / 2$.

Mesh: 4 X 4 unform grid of 9 noded Lagrangian quadratic elements.

Fig. 2.4: Details of a Simply Supported Isotropic Square Plate under Uniform Pressure

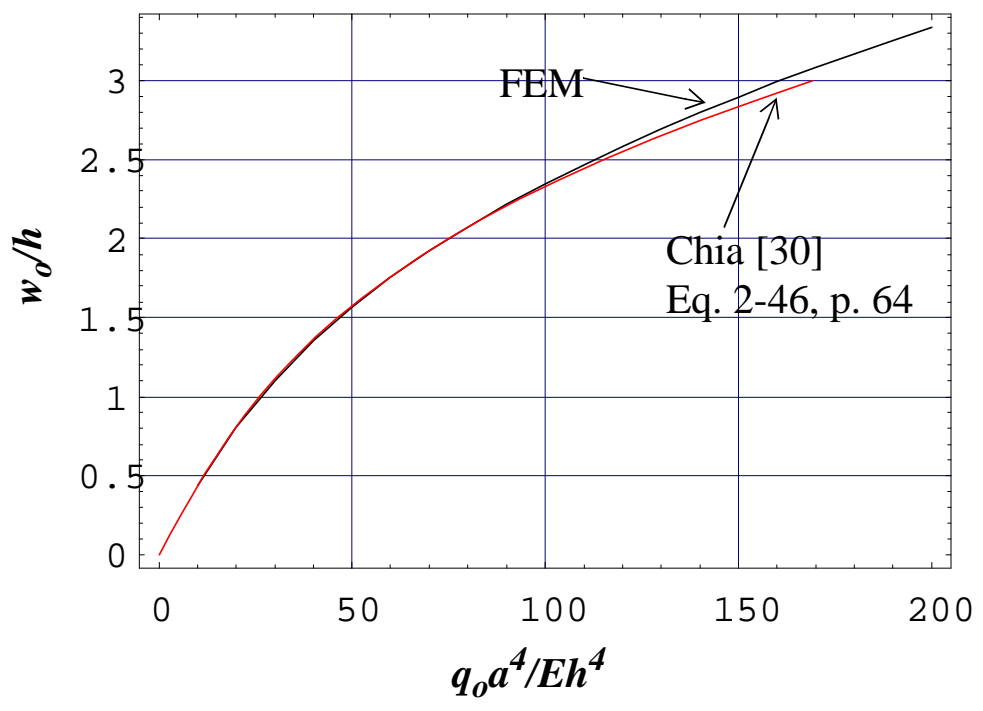

Fig. 2.5: Normalized Plot of Central Deflection versus Uniform Pressure for Simply Supported Isotropic Square Plate 


\subsubsection{Loosely Clamped Isotropic Square Plate}

In this case a square isotropic is plate is clamped along all of its four edges. The plate is clamped in such a way that no external constraints are imposed on the inplane displacements along these edges. Figure 2.6 lists the details of such plate. Figure 2.7 is a comparison of the finite element solution with the one-term Galerkin solution of Chia [30].

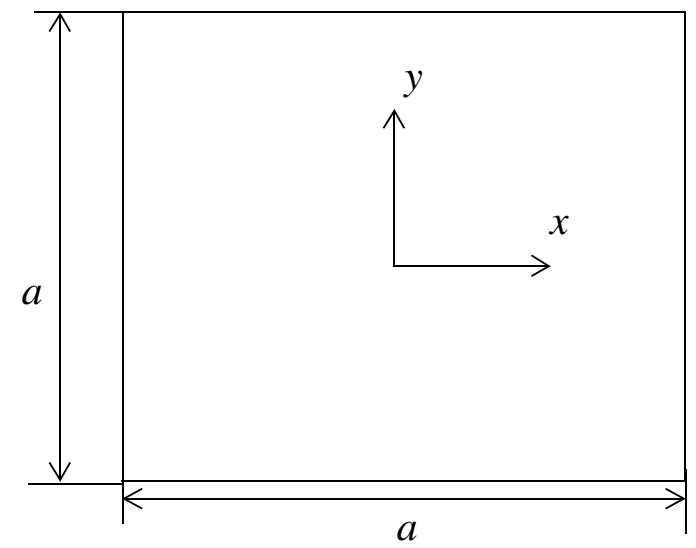

Young's Modulus $(E)=200.0 \mathrm{GPa}$

Poisson's ration $(\mathrm{V})=0.316$

Plate thickness(h): $2 \mathrm{~mm}$.

Boundary Conditions:

$u=0$ at $x=-a / 2, v=0$ at $(x, y)=(-a / 2,-a / 2)$

$w=0$ at $x= \pm a / 2, y= \pm a / 2$.

$\partial w / \partial x=\partial w / \partial y=0$ at $x= \pm a 2 /, y= \pm a / 2$.

Mesh: 4 X 4 unform grid of 9 noded Lagrangian quadratic elements.

Fig. 2.6: Details of a Loosely Clamped Isotropic Square Plate under Uniform Pressure

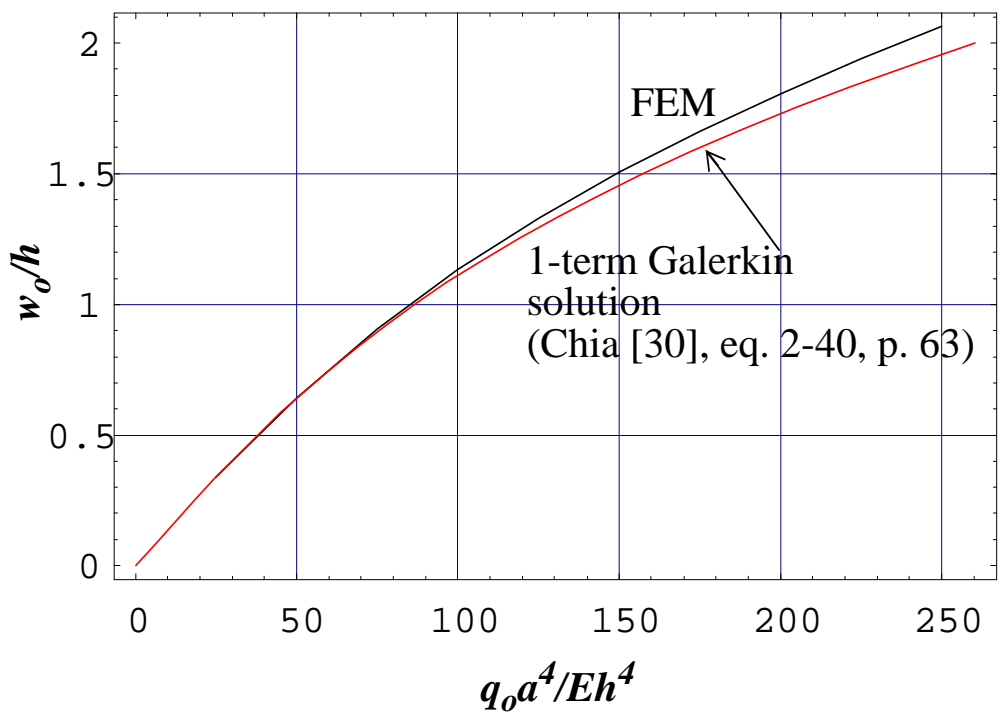

Fig. 2.7: Normalized Plot of Central Deflection versus Uniform Pressure for Loosely Clamped Isotropic Square Plate 


\subsubsection{Rigidly Clamped Isotropic Rectangular Plate}

Finally, a rectangular isotropic plate which is rigidly clamped along all of its four edges and subjected to uniform pressure $q_{o}$ is analyzed as a benchmark. The plate is clamped in such a way that all the inplane and out-of-plane degrees of freedoms are constrained to remain zero along its edges. Figure 2.8 enlists the details of such plate. Figure 2.9 is a comparison of finite element solution with the one term Galerkin solution of Chia [30]. Finite element solutions have been computed for rectangular plates for two aspect ratios.

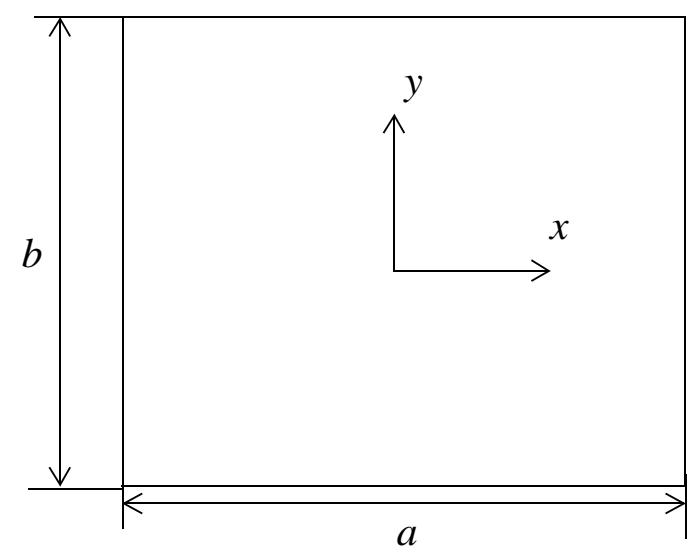

Young's Modulus $(E)=200.0 \mathrm{GPa}$

Poisson's ratio $(\mathrm{V})=0.33$

Plate thickness(h): $2 \mathrm{~mm}$.

Boundary Conditions:

$(u, v, w)=(0,0,0)$ at $x= \pm a / 2, y= \pm a / 2$

$\partial w / \partial x=\partial w / \partial y=0$ at $x= \pm a 2 /, y= \pm a / 2$.

Mesh: 4 X 4 unform grid of 9 noded Lagrangian quadratic elements.

$$
\begin{aligned}
& a_{o}=a / 2 \\
& D=E h^{3} / 12\left(1-v^{2}\right) \\
& \lambda=a / b=1.0,2.0
\end{aligned}
$$

Fig. 2.8: Details of a Rigidly Clamped Isotropic Rectangular

Plate under Uniform Pressure 


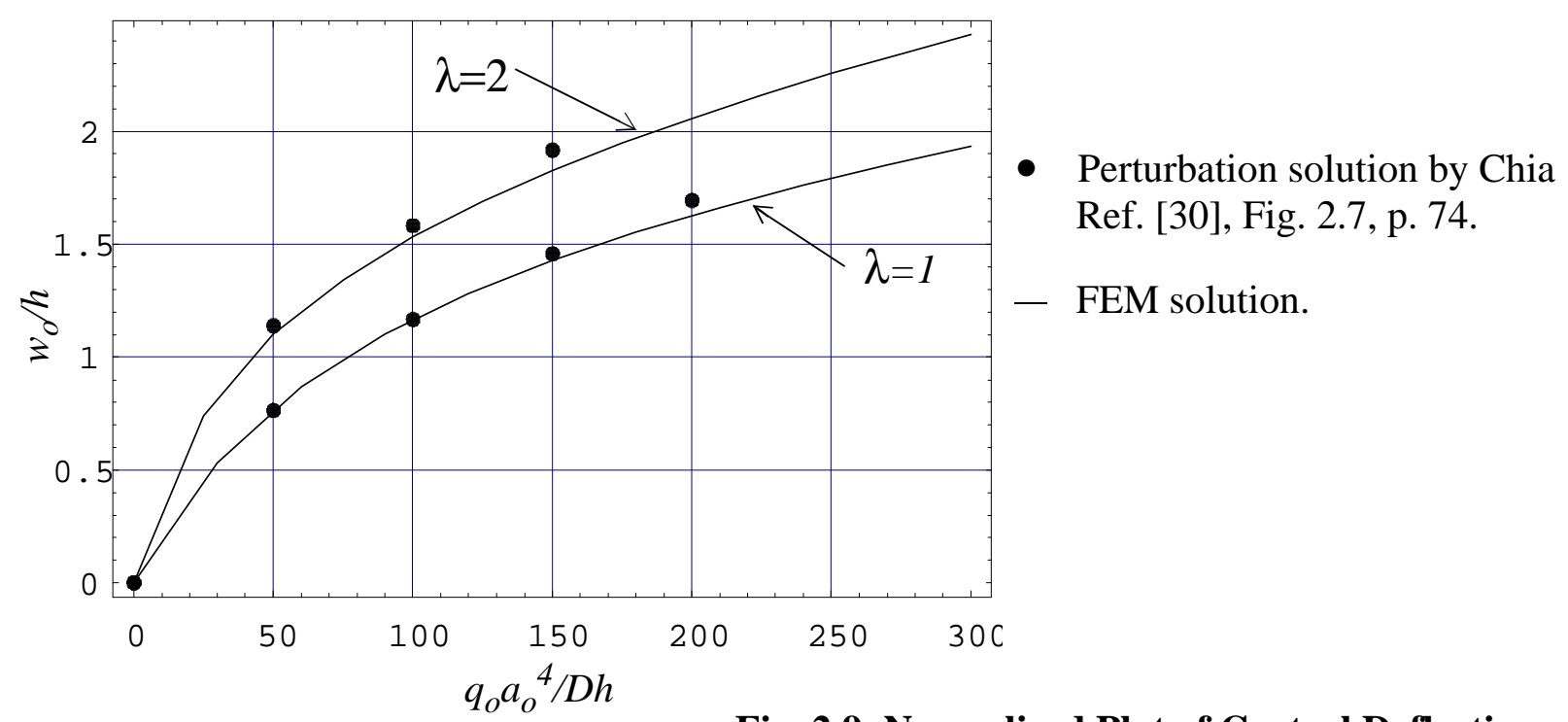

Fig. 2.9: Normalized Plot of Central Deflection versus Uniform Pressure for Rigidly Clamped Isotropic Rectangular Plates

These three cases show that the nonlinear solution as obtained from the finite element program is valid for isotropic plates. There is a high degree of correlation between the analytical results and the finite element results as computed by the algorithm developed. However, for higher level of external pressures, it is seen from Figs. 2.5, 2.7, and 2.9 that there is marginal divergence between finite element predictions and the solutions as developed by Chia. This difference can be attributed to the facts that the mesh size is a relatively coarse one, and also that Chia's results are single term series solutions to the above mentioned problems.

\subsubsection{Symmetric Composite Plates Subjected to Uniform Lateral Pressure}

The following cases test the program's ability to predict the nonlinear behavior of orthotropic as well as anisotropic plates.

\subsubsection{Rigidly Clamped Orthotropic Composite Plate Under Uniform Lateral Pressure}

Consider a rectangular orthotropic plate clamped rigidly along all the four edges. The plate is subjected to uniform lateral pressure $q_{o}$. For computation purposes typical elastic constants of glass- 
epoxy composites have been considered. The material properties are

$$
E_{11}=75 \mathrm{GPa} \quad E_{11} / E_{22}=3.00 \quad G_{12} / E_{11}=0.50 \quad \mathrm{v}_{12}=0.25
$$

These elastic properties imply that the plate is stiffer in the $x$-direction, which is the direction of the fibers. Figure 2.10 shows the details of the plate, and Fig. 2.11 is a comparison of finite element results and the analytical results obtained by Chia [30]. Two plates, a square and a rectangular one, have been considered.

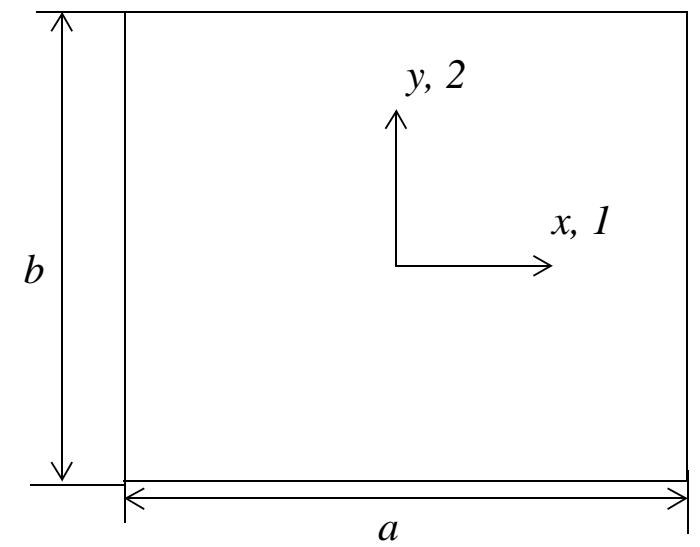

Plate thickness $(h): 2 \mathrm{~mm}$

$a_{o}=a / 2=0.127 \mathrm{~m}, b_{o}=b / 2$

$\lambda=a_{o} / b_{o}=1.0,0.75$.

Boundary Conditions:

$(u, v, w)=(0,0,0)$ at $x= \pm a / 2, y= \pm a / 2$

$\partial w / \partial x=\partial w / \partial y=0$ at $x= \pm a / 2, y= \pm a / 2$.

Mesh: 4 X 4 unform grid of 9 noded Lagrangian quadratic elements.

\section{Fig. 2.10: Rigidly Clamped Orthotropic Plate Subjected to Uniform Pressure}

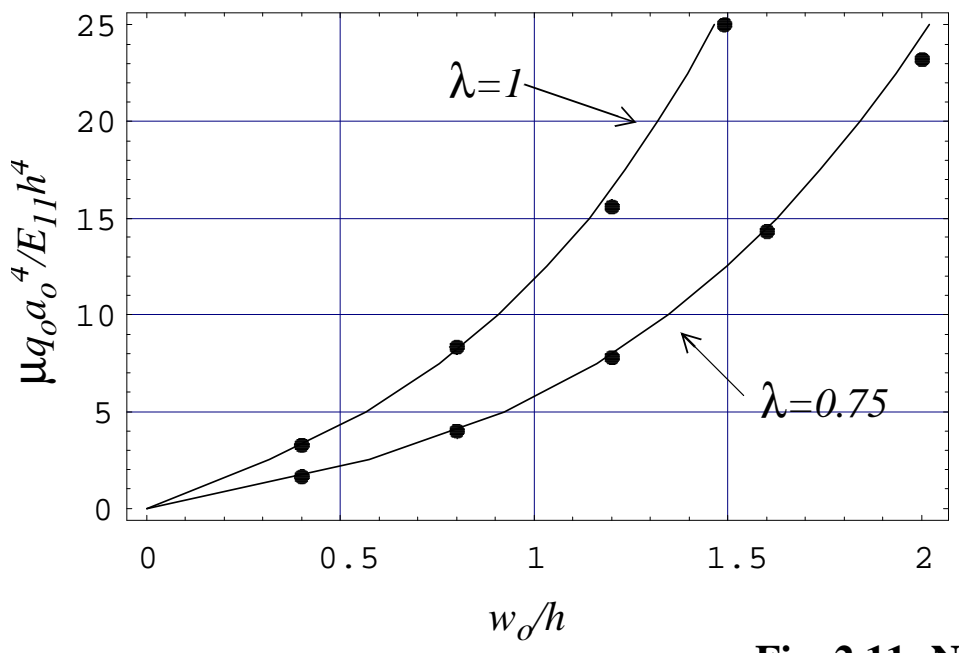

- Perturbation solution by Chia Ref. [30], Fig. 5.2, p. 227.

- FEM solution.

$$
\mu=1-v_{12} v_{21}
$$

Fig. 2.11: Normalized Plot of Central Deflection versus Uniform Pressure for Rigidly Clamped Orthotropic Plates 


\subsubsection{Loosely Clamped Anisotropic Unsymmetrically Laminated Composite Plates Sub- jected to Uniform Lateral Pressure}

Here two square plates have been considered. Both of these plates are loosely clamped on the edges and subjected to uniform lateral pressure $q_{o}$. The plates are unsymmetrically laminated and the layer orientation is $\left[+\theta /-\left(\theta+90^{\circ}\right)\right]$. Two values of $\theta$ have been considered, $0^{\circ}$ and $45^{\circ}$. The material properties are

$$
E_{11}=200 \mathrm{GPa} \quad E_{11} / E_{22}=40.0 \quad G_{12} / E_{22}=0.50 \quad \mathrm{v}_{12}=0.25
$$

The boundary conditions for the plate are given in Fig. 2.6. The dimensions of the plate are 0.254 $\mathrm{m} \mathrm{X} 0.254 \mathrm{~m}$. Figure 2.12 is a comparison of the response of these plates as predicted by the finite element program with that predicted by Chia [30].

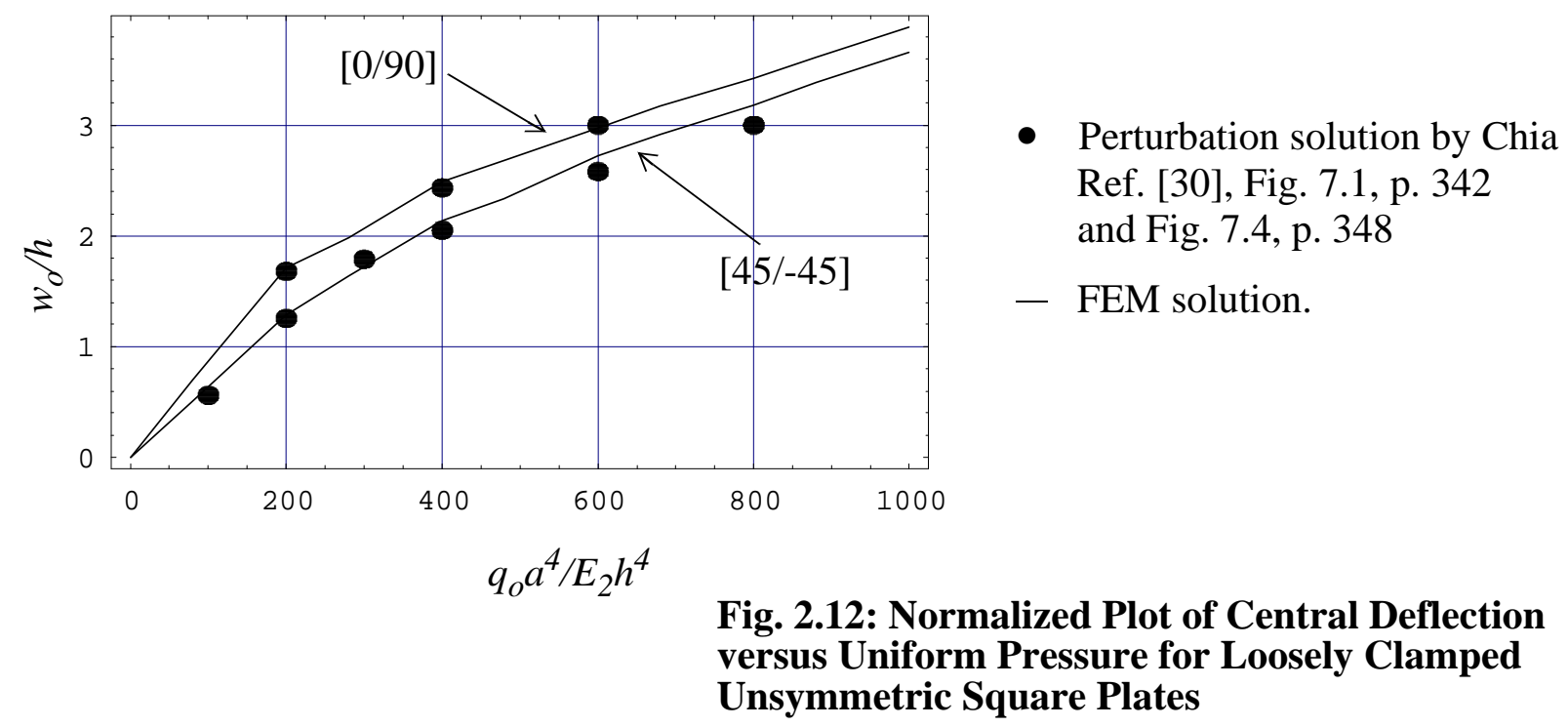

The two cases discussed above test the program's ability to handle anisotropic laminated plates which are not necessarily symmetric about their midplane. It is seen from these test cases that the finite element program can predict their behaviors with a high degree of accuracy. 


\subsubsection{Unsymmetrically Laminated Linear Plate Subjected to Uniform Temperature Change}

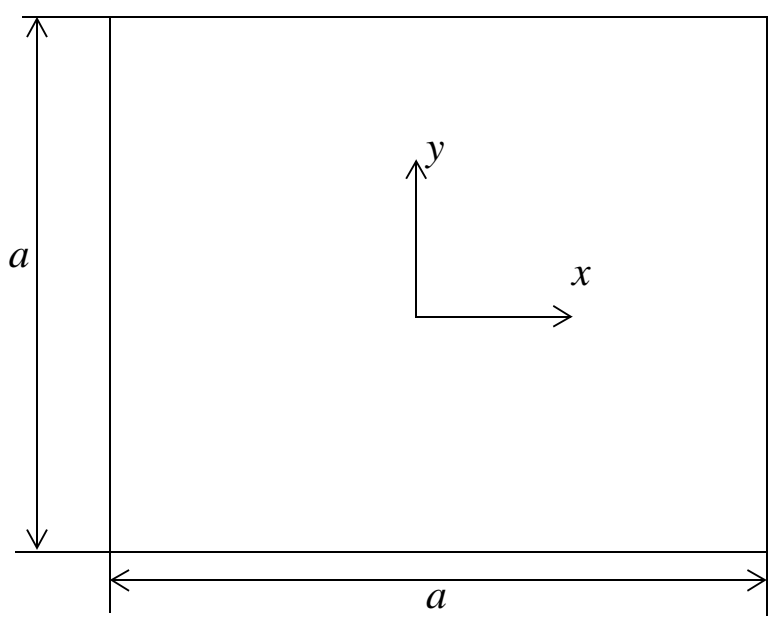

Fig. 2.13: Unsymmetrically Laminated Plate Subjected to Uniform Temperature Change
Material Properties:

$E_{11}=19.755 \mathrm{GPa}$

$E_{22}=1.9755 \mathrm{GPa}$

$G_{12}=0.70 \mathrm{GPa}$

$\mathrm{v}_{12}=0.35$

$\alpha_{11}=7 \times 10^{-6}{ }^{\rho} \mathrm{C} \alpha_{22}=23 \times 10^{-6}{ }^{\rho} \mathrm{C}$

Layup: $\left[0_{5} / 45_{3}\right]$

Thickness of each layer $=1 \mathrm{~mm}$. $a=0.25 \mathrm{~m}$.

Boundary Conditions:

$$
u=v=w=\psi^{o}{ }_{x}=0 \text { at }(x, y)=(0,0)
$$$$
\Delta T=-100^{\circ} \mathrm{C}
$$

Mesh: 10 X 10 grid of 9 noded

Lagrangian quadratic elements.

This sections explores the ability of the program developed to handle problems involving temperature changes, and the consequential thermal forces and moments. The details of the plate are given in Fig. 2.13. Such a plate has a uniform strain and curvature field. The response can be predicted by classical lamination theory and can be expressed as

$$
\left[\begin{array}{c}
\varepsilon_{x x}^{o} \\
\varepsilon_{y y}^{o} \\
\gamma_{x y}^{o} \\
\kappa_{x x}^{o} \\
\kappa_{y y}^{o} \\
\kappa_{x y}^{o}
\end{array}\right]=\left[\begin{array}{llllll}
A_{11} & A_{12} & A_{16} & B_{11} & B_{12} & B_{16} \\
A_{12} & A_{22} & A_{26} & B_{12} & B_{22} & B_{26} \\
A_{16} & A_{26} & A_{66} & B_{16} & B_{26} & B_{66} \\
B_{11} & B_{12} & B_{16} & D_{11} & D_{12} & D_{16} \\
B_{12} & B_{22} & B_{26} & D_{12} & D_{22} & D_{26} \\
B_{16} & B_{26} & B_{66} & D_{16} & D_{26} & D_{66}
\end{array}\right]^{-1}\left[\begin{array}{r}
N_{x}^{T} \\
N_{y}^{T} \\
N_{x y}^{T} \\
M_{x}^{T} \\
M_{x}^{T} \\
M_{x y}^{T}
\end{array}\right] \Delta T
$$


The following table compares the results obtained from the above equation with those obtained from the finite element code developed.

Table 2.2: FEM and Exact Results for an Unsymmetrically Laminated Plate Subjected to Uniform Temperature Field (values in microstrains and microradians).

\begin{tabular}{|l|l|l|l|l|l|l|}
\hline & $\varepsilon_{x x}^{o} / \Delta T$ & $\varepsilon_{y y}^{o} / \Delta T$ & $\gamma_{x y}^{o} / \Delta T$ & $\kappa_{x x}^{o} / \Delta T$ & $\kappa_{y y}^{o} / \Delta T$ & $\kappa_{x y}^{o} / \Delta T$ \\
\hline \hline Classical Theory & -8.14 & -20.20 & -6.99 & 580.0 & -1000.0 & -2345.0 \\
\hline FEM & -8.13 & -20.2 & 7.00 & 584.0 & -1000.0 & -2340.0 \\
\hline
\end{tabular}

\subsection{CLOSURE}

A finite element code in the FORTRAN programing language has been developed. The program captures the effects of anisotropy, geometric nonlinearity due to moderate rotations, first-order shear deformation, and thermo-elasticity in thin rectangular panels subjected to conservative external forces. The code employs the method of Newton-Raphson for the purpose of solving the ensuing nonlinear simultaneous algebraic equations. The accuracy of this finite element code has been explored and it has been found that the calculations are in agreement with the results found in available literature. 


\section{NUMERICAL PREDICTIONS FOR SECONDARY BUCKLING}

\subsection{INTRODUCTION}

This chapter focuses on the numerical results obtained from the finite element code developed. These results pertain to the finite element analysis of plates which are clamped along the loading edges and simply supported along the other two edges. Studies have been conducted to explore the effects of variations in lamination sequence and inplane boundary conditions along the simply supported edges, the presence of stiffeners, and the role of imperfections on secondary buckling. Further, studies have also been conducted to explore the role played by out-of-plane shear deformations on the deep postbuckling response of anisotropic plates. Finally, secondary buckling of metallic plates has been studied and compared with composite plates.

\subsection{DETAILS OF THE CLAMPED-SIMPLY SUPPORTED PLATES STUDIED}

A simplified representation of the plate which has been analyzed is shown in Fig. 3.1. As depicted in Fig. 3.1, the plate is subjected to a uniform inplane end shortening displacement $\Delta$ applied at the top end of the plate. Of course, an inplane load $P$ is required to effect this end shortening. The plate is stiffened on the right and left edges by steel stiffeners. There are two stiffeners along each simply supported edge, one on the top surface and the other on the bottom surface. Steel was chosen as the material for ease of fabrication of specimens for experiments. The stiffness of these stiffeners could be easily controlled by varying the thickness and the width of the steel strips. The right and left edges of the plate were supported by a line of simple supports, halfway across the width of the stiffeners, and the top and bottom ends were clamped. The requirement of a finite area for clamping in the experiments is reflected in the figure. It is impossible to design a fixture to clamp, i.e., restrict rotations, along just a line. The overall dimensions of the plate were $a$ by $b$. The width of the clamped areas on each end was $c$ and the stiffeners had a width and thickness of $d$ and $t$, respectively. The overall thickness of the plate was $h$. The figure shows that a small portion of the plate and stiffener extend beyond the simply supported edges. This extra width for the 
plate was necessary to accommodate the decrease in plate width in its postbuckling configurations. Care was taken to include the effect of this extra width in the finite element calculations. It must be noted that these extensions of the plate provide extra stiffness to the plate, and also are regions of high inplane compressive stresses. For analysis purposes, the coordinate system is located at the lower left corner of the plate shown in Fig. 3.1.

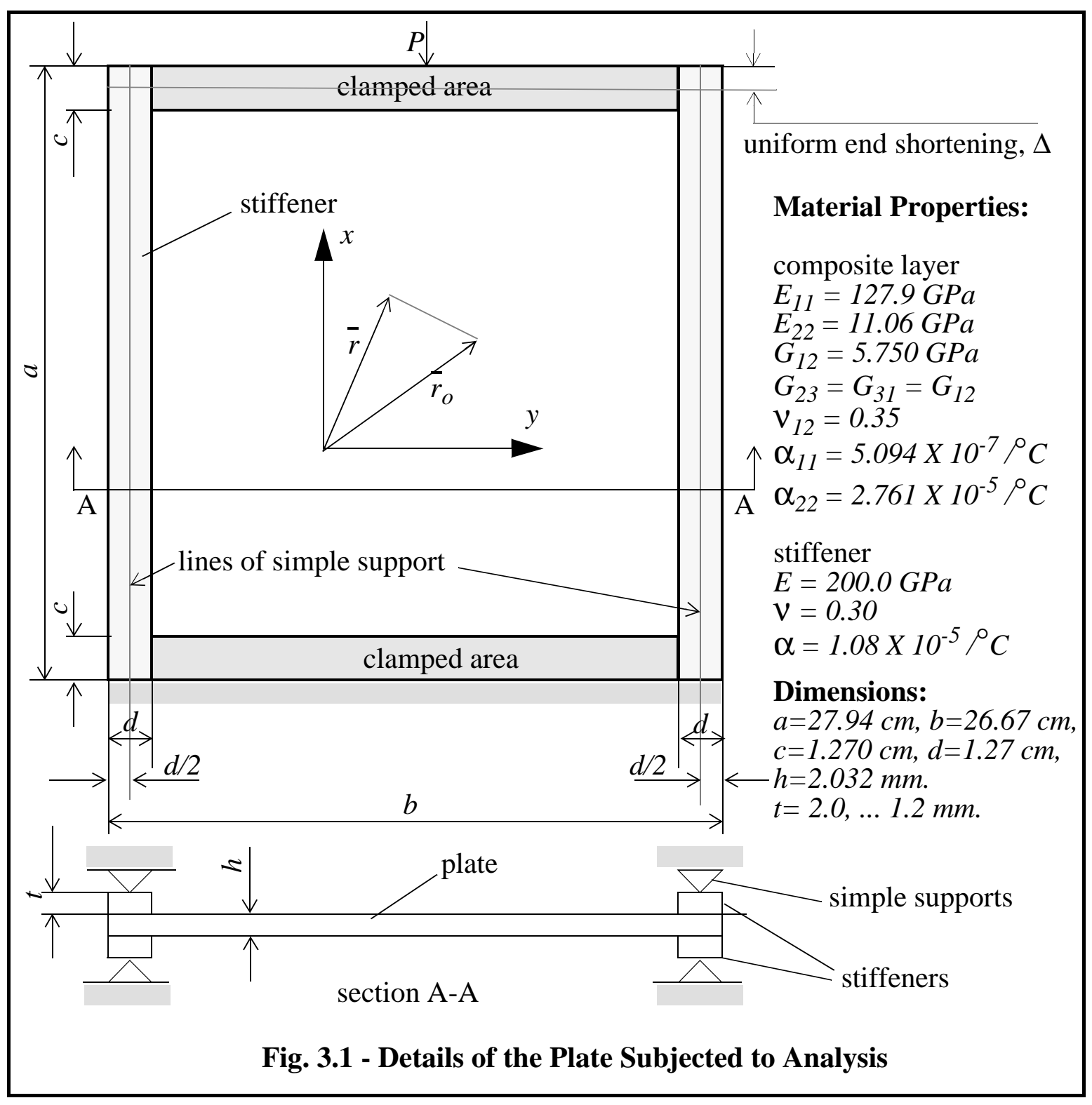

Tables 3.1, 3.2, and 3.3 give the details of the 57 case studies which have been analyzed. These tables also detail the associated nomenclature which has been used in the presentation of the numerical results, and the ensuing discussions. The boundary conditions, which are common to 
all these cases are, $w=0$ along the simply supported edges and in the clamped area, $u=0$ at $x=0$, and $u=-\Delta$ at $x=a$. Table 3.1 lists the 24 cases of perfectly flat plates which have been analyzed. These case studies have a generic name of ${ }^{\alpha} \mathrm{P}_{\beta}$. Here, $\mathrm{P}$ indicates that the plate is perfectly flat. The superscript $\alpha$ is indicative of the layup, where $\alpha$ could assume three values, $0, q$, and 90 . These stand for $\left[ \pm 45 / 0_{2}\right]_{2 s},[ \pm 45 / 0 / 90]_{2 s}$ and $\left[ \pm 45 / 90_{2}\right]_{2 s}$ layups, respectively. The direction of loading in these plates is along the $0^{\circ}$ direction. While the $\left[ \pm 45 / 0_{2}\right]_{2 \mathrm{~s}}$ and $\left[ \pm 45 / 90_{2}\right]_{2 \mathrm{~s}}$ plates are stiff in the loading direction and the transverse directions, respectively, the $[ \pm 45 / 0 / 90]_{2 s}$ plates have inplane isotropy characteristics. The subscript $\beta$ is indicative of the boundary conditions along the simply supported edges in the $y$ direction and can assume values between 1 and 8 . The value of $\beta=1$ means the simply supported edge is restrained from having any inplane displacements normal to the edge. A value of 3 means the simply supported edge is traction-free, while a value of 2 means the inplane displacement normal to the edge is a constant, i.e. does not vary with $x$, and the net force normal to the edge, $\int N_{y} d x$, is zero. This net force condition provides a means to evaluate the value of the constant. The values of $\beta$ from 4 to 8 are related to traction-free conditions and stiffeners of various thicknesses.

Table 3.2 is a listing of 24 plates which are imperfect analogs of the perfect plates. These cases have a generic name of ${ }^{\alpha} \mathrm{I}_{\beta}$, I being indicative of the out-of-plane imperfections in these plates. Table 3.3 lists the remaining 9 plates. Three of these have a generic name of ${ }^{m_{P}}{ }_{\beta}$, where the superscript $m$ indicates that the plate is metallic and made of aluminum. The remaining six plates listed in Table 3.3 have a generic name of ${ }^{n} S_{\beta}$, where $S$ denotes these plates help us understand the influence of shear-deformations on secondary buckling, and $n$ is indicative of the ratio of outof-plane shear moduli, $G_{23}$ and $G_{31}$, to inplane shear modulus $G_{12}$.

\begin{tabular}{|c|c|c|c|c|c|c|c|c|}
\hline \multicolumn{9}{|c|}{ Table 3.1: Nomenclature for Perfectly Flat Plates } \\
\hline $\mathrm{BC}$ along SS & \multicolumn{3}{|c|}{ Unstiffened Plates } & \multicolumn{5}{|c|}{$\begin{array}{c}\text { Stiffened Plates with } N_{y}=0 \text { along SS Edge } \\
\text { Stiffener Thickness } t \text { mm }\end{array}$} \\
\hline Layup & $v=0$ & $\begin{array}{l}\int N_{y} d x=0 \\
v=\text { const. }\end{array}$ & $N_{y}=0$ & 2.0 & 1.8 & 1.6 & 1.4 & 1.2 \\
\hline$\left[ \pm 45 / 0_{2}\right]_{2 \mathrm{~s}}$ & ${ }^{0} \mathrm{P}_{1}$ & ${ }^{0} \mathrm{P}_{2}$ & ${ }^{0} \mathrm{P}_{3}$ & ${ }^{0} \mathrm{P}_{4}$ & ${ }^{0} \mathrm{P}_{5}$ & ${ }^{0} \mathrm{P}_{6}$ & ${ }^{0} \mathrm{P}_{7}$ & ${ }^{0} \mathrm{P}_{8}$ \\
\hline$[ \pm 45 / 0 / 90]_{2 \mathrm{~s}}$ & ${ }^{\mathrm{q}} \mathrm{P}_{1}$ & ${ }^{\mathrm{q}} \mathrm{P}_{2}$ & ${ }^{\mathrm{q}} \mathrm{P}_{3}$ & ${ }^{\mathrm{q}} \mathrm{P}_{4}$ & ${ }^{\mathrm{q}} \mathrm{P}_{5}$ & ${ }^{\mathrm{q}} \mathrm{P}_{6}$ & ${ }^{\mathrm{q}} \mathrm{P}_{7}$ & ${ }^{\mathrm{q}} \mathrm{P}_{8}$ \\
\hline$\left[ \pm 45 / 90_{2}\right]_{2 \mathrm{~s}}$ & ${ }^{90} \mathrm{P}_{1}$ & ${ }^{90} \mathrm{P}_{2}$ & ${ }^{90} \mathrm{P}_{3}$ & ${ }^{90} \mathrm{P}_{4}$ & ${ }^{90} \mathrm{P}_{5}$ & ${ }^{90} \mathrm{P}_{6}$ & ${ }^{90} \mathrm{P}_{7}$ & ${ }^{90} \mathrm{P}_{8}$ \\
\hline
\end{tabular}




\begin{tabular}{|c|c|c|c|c|c|c|c|c|}
\hline \multirow{2}{*}{$\begin{array}{c}\text { BC along SS } \\
\text { Edge } \Rightarrow \\
\text { Layup }\end{array}$} & \multicolumn{3}{|c|}{ Unstiffened Plates } & \multicolumn{5}{|c|}{$\begin{array}{c}\text { Stiffened Plates with } N_{y}=0 \text { along SS Edge } \\
\text { Stiffener Thickness } t \mathrm{~mm}\end{array}$} \\
\hline & $v=0$ & $\begin{array}{l}\int N_{y} d x=0 \\
v=\text { const. }\end{array}$ & $N_{y}=0$ & 2.0 & 1.8 & 1.6 & 1.4 & 1.2 \\
\hline$\left[ \pm 45 / 0_{2}\right]_{2 \mathrm{~s}}$ & ${ }^{0} \mathrm{I}_{1}$ & ${ }^{0} \mathrm{I}_{2}$ & ${ }^{0} \mathrm{I}_{3}$ & ${ }^{0} \mathrm{I}_{4}$ & ${ }^{0} \mathrm{I}_{5}$ & ${ }^{0} \mathrm{I}_{6}$ & ${ }^{0} \mathrm{I}_{7}$ & ${ }^{0} \mathrm{I}_{8}$ \\
\hline$[ \pm 45 / 0 / 90]_{2 \mathrm{~s}}$ & ${ }^{\mathrm{q}} \mathrm{I}_{1}$ & ${ }^{\mathrm{q}} I_{2 I}$ & ${ }^{\mathrm{q}} I_{3}$ & ${ }^{\mathrm{q}} \mathrm{I}_{4}$ & ${ }^{\mathrm{q}} \mathrm{I}_{5}$ & ${ }^{{ }^{\mathrm{q}}} \mathrm{I}_{6}$ & ${ }^{\mathrm{q}} \mathrm{I}_{7}$ & ${ }^{\mathrm{q} \mathrm{I}_{8}}$ \\
\hline$\left[ \pm 45 / 90_{2}\right]_{2 \mathrm{~s}}$ & ${ }^{90} \mathrm{I}_{1}$ & ${ }^{90} \mathrm{I}_{2}$ & ${ }^{90} \mathrm{I}_{3}$ & ${ }^{90} \mathrm{I}_{4}$ & ${ }^{90} \mathrm{I}_{5}$ & ${ }^{90} \mathrm{I}_{6}$ & ${ }^{90} \mathrm{I}_{7}$ & ${ }^{90} \mathrm{I}_{8}$ \\
\hline
\end{tabular}

\begin{tabular}{|c|c|c|c|}
\hline \multicolumn{2}{|c|}{ Table 3.3: Nomenclature for Unstiffened Metallic and Out-of-Plane Shear Stiff Plates } \\
\hline \hline \multirow{2}{*}{ BC along SS Edge } & Metallic Plates & \multicolumn{2}{|c|}{ Quasi-isotropic Plate } \\
$\boldsymbol{G}_{\mathbf{2} 3}=\boldsymbol{G}_{\boldsymbol{3 1}}=\boldsymbol{n} \boldsymbol{G}_{\boldsymbol{1 2}}$
\end{tabular}

In sections 3.3 through 3.7 results of these 57 case studies have been discussed in detail. Section 3.3 is a discussion of the influence of boundary conditions and stiffener thickness on the postbuckling response of the plates subjected to compressive loads. Section 3.4 details the effect of lamination sequence on the postbuckling characteristics of unstiffened plates. In section 3.5 the interaction of stiffener thickness and lamination sequence, and their accumulated influence on the postbuckling characteristics of plates, will be discussed. The influence of imperfections on the secondary buckling characteristics will be discussed in section 3.4.2. Section 3.6 compares the secondary buckling responses of unstiffened metallic plates with those of quasi-isotropic unstiffened plates. Finally, section 3.7 explores the influences of out-of-plane shear deformations on the secondary buckling characteristics of unstiffened quasi-isotropic plates.

In all these sections, three parameters have been used to normalize the characteristic responses of the plates. These parameters are based on the classic plate ${ }^{\mathrm{q}} \mathrm{P}_{3}$. The normalizing parameters are: plate thickness $h$; the inplane end shortening buckling displacement $\Delta_{c r}$; and, the accompanying 
compressive load $P_{c r}$. The numerical values of these parameters for this plate are: $2.032 \mathrm{~mm}$; $0.1165 \mathrm{~mm}$; and, $11.53 \mathrm{kN}$, respectively. The choice of these parameters is based on the fact that the inplane boundary conditions along the simply supported edges of the plate ${ }^{\mathrm{q}} \mathrm{P}_{3}$ are most easily reproducible in the laboratory.

\subsection{INFLUENCE OF BOUNDARY CONDITIONS AND STIFFENER THICKNESS ON THE BEHAVIOR OF QUASI-ISOTROPIC PLATES}

The section describes and compares the sensitivity of the postbuckling response of perfectly flat quasi-isotropic plates to variations in inplane boundary conditions and stiffener thickness. Figures $3.2 \mathrm{a}$ and $3.2 \mathrm{~b}$ depict the configurations of unstiffened plates before and after the event of secondary buckling. Figures 3.3 and 3.5 are plots of normalized out-of-plane displacement at the center of the plate, $w_{\delta} / h$, versus normalized end shortening, $\Delta \Delta_{c r}$, for the quasi-isotropic plates. Figures 3.4 and 3.6 show the relationship between the normalized applied end shortening and the normalized load $P$ required to produce it. Figure 3.7 shows the loads borne by stiffeners in the stiffened quasi-isotropic plates. Figures $3.8 \mathrm{~b}$ and $3.8 \mathrm{c}$ show the variation of $v$ displacement along the simply supported edges of quasi-isotropic plates before and after secondary buckling. Figures 3.9a and $3.9 \mathrm{~b}$ show the configuration of quasi-isotropic stiffened plates before and after secondary buckling.

\subsubsection{Unstiffened Quasi-isotropic Plates}

Among the unstiffened quasi-isotropic plates, ${ }^{\mathrm{q}} \mathrm{P}_{1}$ and ${ }^{\mathrm{q}} \mathrm{P}_{3}$ have a real basis in the sense that their boundary conditions along the simply supported edge can be closely approximated in the laboratory. Plate ${ }^{\mathrm{q}} \mathrm{P}_{2}$ is a case that is difficult to simulate in the laboratory and has been studied because it represents a limiting case. Specifically, case ${ }^{\mathrm{q}} \mathrm{P}_{2}$ represents a stiffener with infinite resistance to differences in the $v$ displacement along the simply supported edges, i.e., $\partial v / \partial x$ is forced to be zero there, but it offers no resistance to other displacements. It can seen in Fig. 3.3 that, as expected, as $\Delta$ increases from zero, plate ${ }^{\mathrm{q}} \mathrm{P}_{3}$ remains flat prior to buckling, namely, $w_{\delta} / h$ remains zero until $\Delta / \Delta_{\text {cr }}$ becomes unity. For $\Delta$ greater than $\Delta_{c r}$, the plate deflects out of plane, $w_{o}$ becoming a nonzero value. Just after buckling, the configuration of the plate is as shown in Fig. 3.2a, 
having one half-wave in both the $x$ and $y$ directions. The out-of-plane deflection continues to grow monotonically with increasing end shortening, the out-of-plane deflection reaching over 4 plate thicknesses when the end shortening is 8 times its buckling value. Referring to Fig. 3.4, while the plate is flat, the slope of the relation represents the inplane stiffness of the plate. After the plate has buckled, the slope of the relation decreases sharply and continues to decrease with increasing end shortening.

To a certain level of end shortening, the pre- and postbuckling responses of plates ${ }^{\mathrm{q}} \mathrm{P}_{1}$ and ${ }^{\mathrm{q}} \mathrm{P}_{2}$ are similar to that of ${ }^{\mathrm{q}} \mathrm{P}_{3}$. It can be seen, however, that the buckling end shortening for ${ }^{\mathrm{q}} \mathrm{P}_{1}$ is less than that for ${ }^{\mathrm{q}} \mathrm{P}_{3}$. This is a consequence of the restraint on the Poisson-induced displacement, $v$, along the simply supported edges. This restraint makes the loading biaxial and it therefore buckles at lower values of $\Delta$ and $P$ than plate ${ }^{\mathrm{q}} \mathrm{P}_{3}$. A more important difference, however, can be seen when the deep postbuckling responses $\left(\Delta / \Delta_{\mathrm{cr}}>4.5\right)$ of these plates are compared. Unlike plate ${ }^{\mathrm{q}} \mathrm{P}_{3}$, both plates ${ }^{\mathrm{q}} \mathrm{P}_{1}$ and ${ }^{\mathrm{q}} \mathrm{P}_{2}$ exhibit secondary buckling, that is, the configuration of these plates suddenly changes from one with one half-wave in the $x$ direction to one with two half-waves in the $x$ direction, as depicted in Fig. 3.2b. As a consequence, $w_{O}$ dramatically drops to zero and the maximum deflection of the plate occurs nearer the clamped ends and has less magnitude. This sudden drop of $w_{o}$ to zero is shown in Fig. 3.3. Further, as seen in Fig. 3.4, secondary buckling is also accompanied by a corresponding sharp drop in the load necessary to produce the end shortening. It is important to recognize that this jump from the configuration of Fig. 3.2a to the configuration of Fig. 3.2b is a dynamic event. The dynamics of this event have not been modeled here, rather the transition between the two configurations is denoted as simply a straight vertical line. Inertia effects, damping, and whatever perturbation conditions cause the plate to begin the transition from one configuration to the other all have an influence on the exact characteristic of the transition.

Figures 3.3 and 3.4 provide an understanding of the effect of boundary conditions on the occurrence of secondary buckling and the stability of the plate in deep postbuckling range. It appears from these figures that any constraints on the $v$ displacement field which exist along the simply supported edges of the plate tend to destabilize the plate in the deep postbuckling range. A comparison of the responses for plates ${ }^{\mathrm{q}} \mathrm{P}_{1}$ and ${ }^{\mathrm{q}} \mathrm{P}_{2}$ shows that the severity of the boundary conditions 
along the simply supported edges affects the onset of the secondary buckling event.

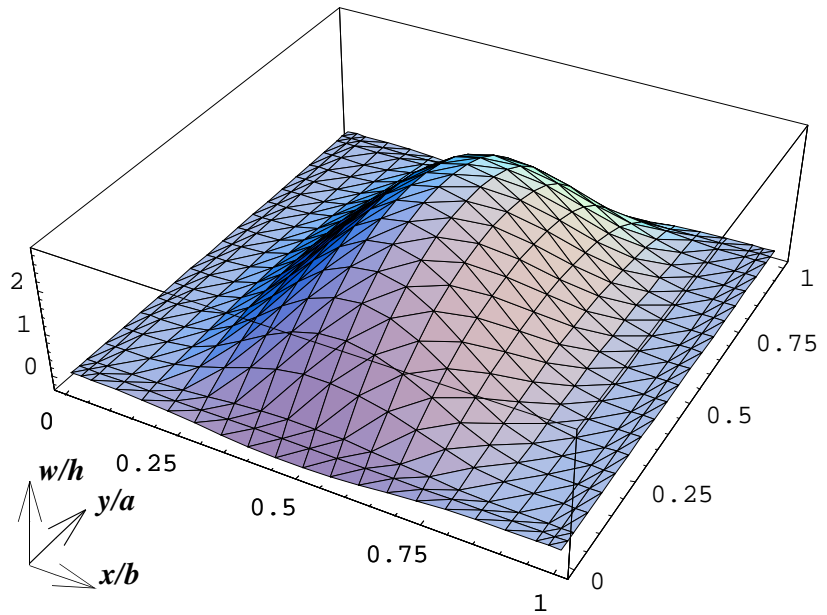

Fig. 3.2a: Postbuckling Plate Configuration before Secondary Buckling

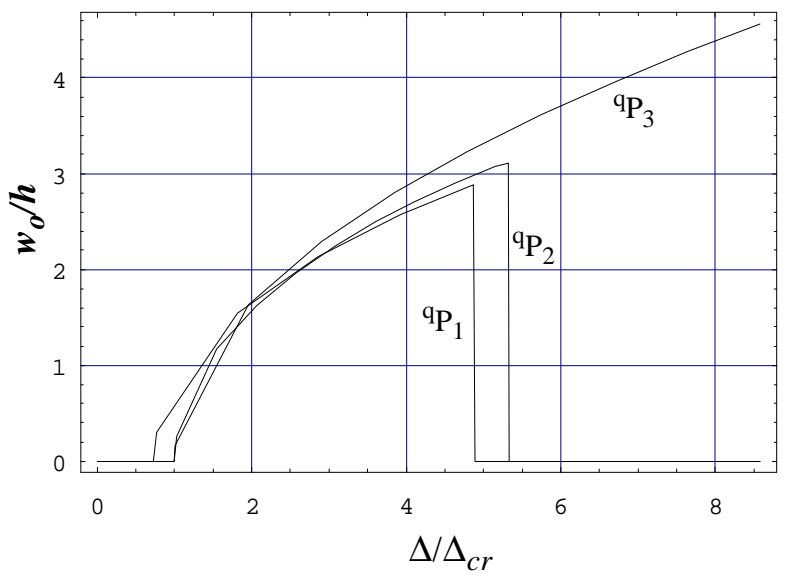

Fig. 3.3: Deflection Response of Perfectly Flat Unstiffened Plates

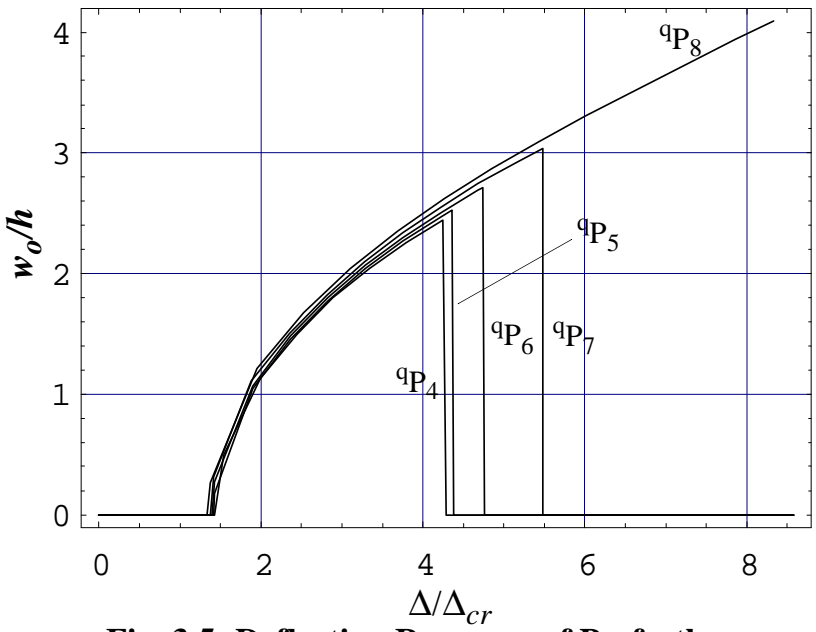

Fig. 3.5: Deflection Response of Perfectly Flat Stiffened Plates

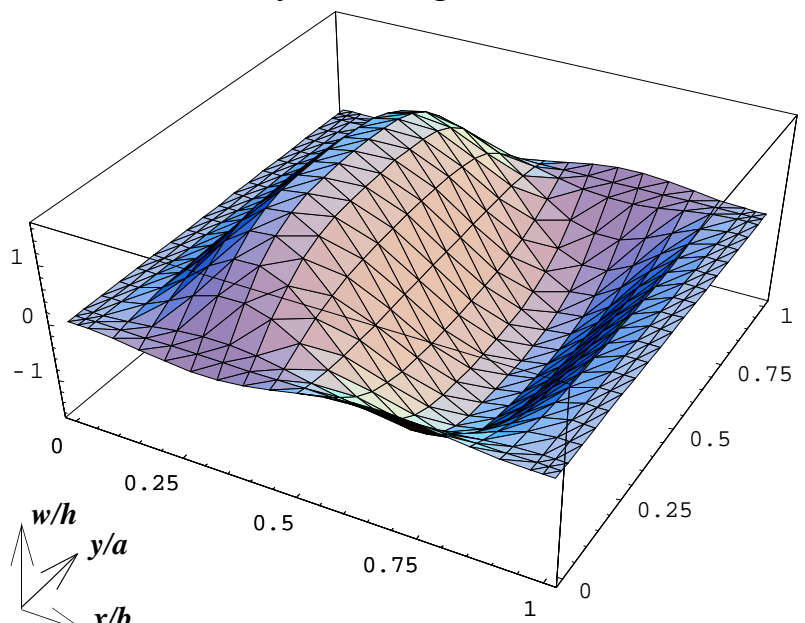

Fig. 3.2b: Postbuckling Plate Configuration after Secondary Buckling

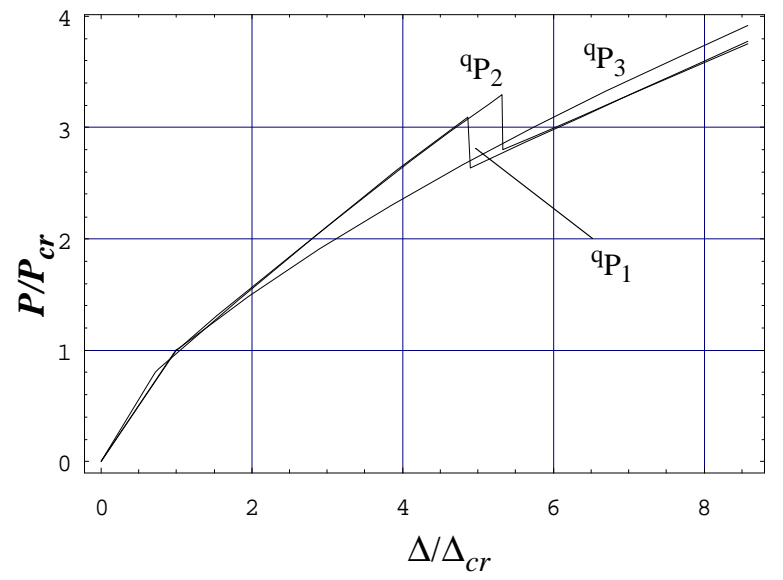

Fig. 3.4: Load Response of Perfectly Flat Unstiffened Plates

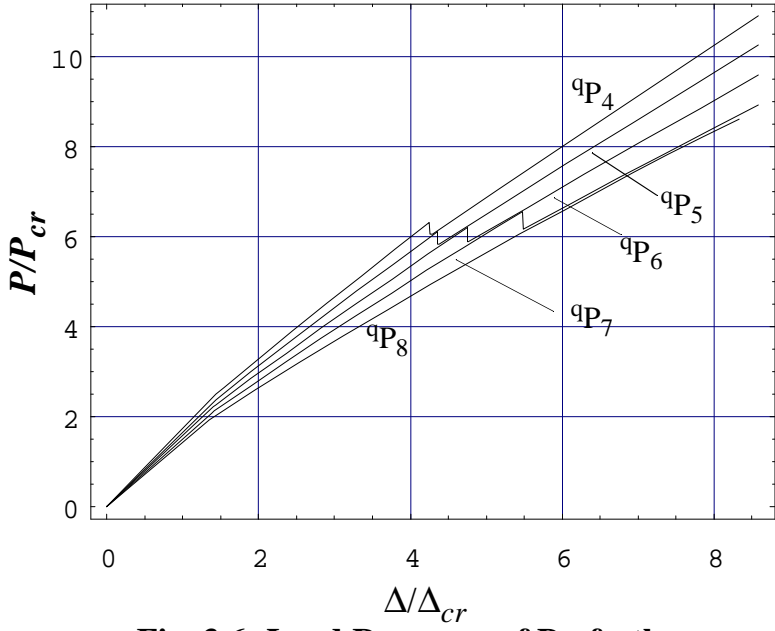

Fig. 3.6: Load Response of Perfectly Flat Stiffened Plates 


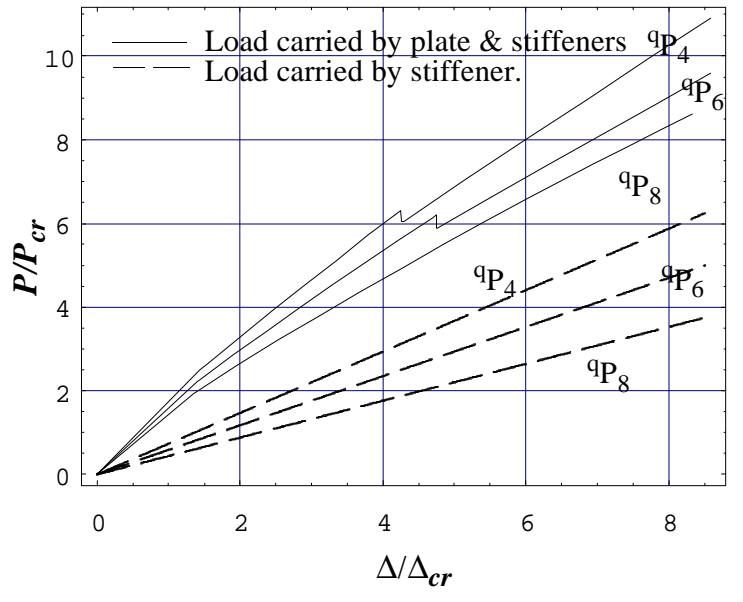

Fig. 3.7: Load Sharing between Stiffeners and Plate

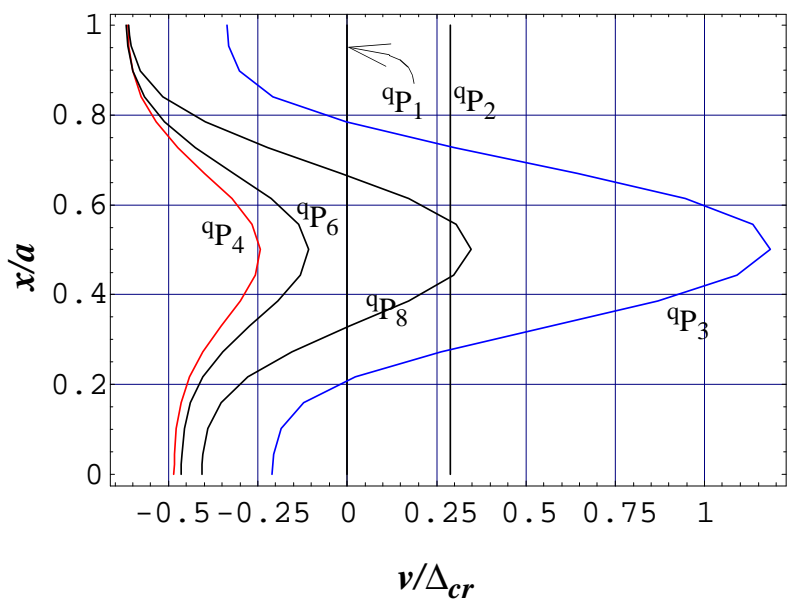

Fig. 3.8b: Variation of $v$ Displacement prior to Secondary Buckling

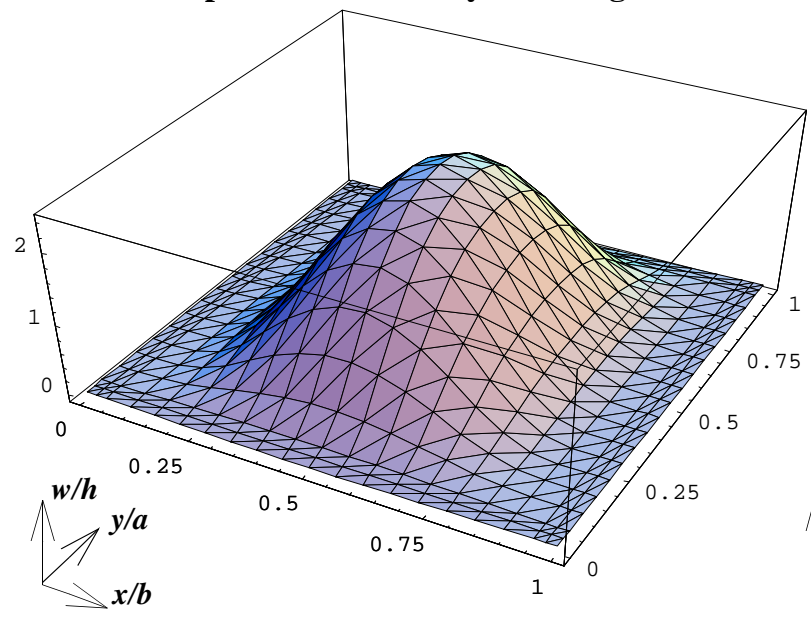

Fig. 3.9a: Configuration of Stiffened Plate before Secondary Buckling

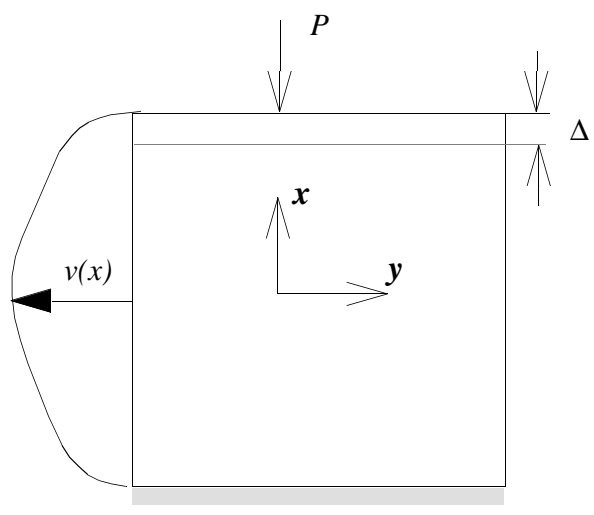

Fig. 3.8a: Illustration of $v$ Displacement which has been Plotted in Figs. 3.8b, 3.8c

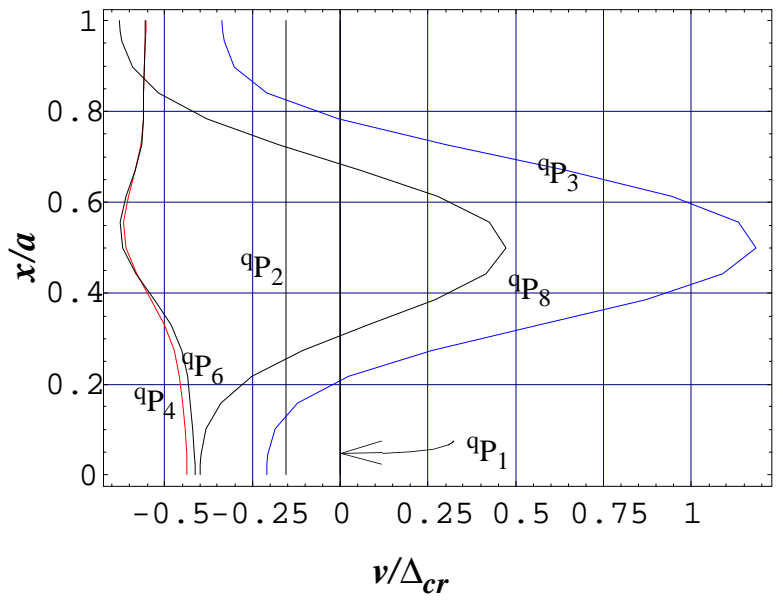

Fig. 3.8b: Variation of $v$ Displacement after Secondary Buckling

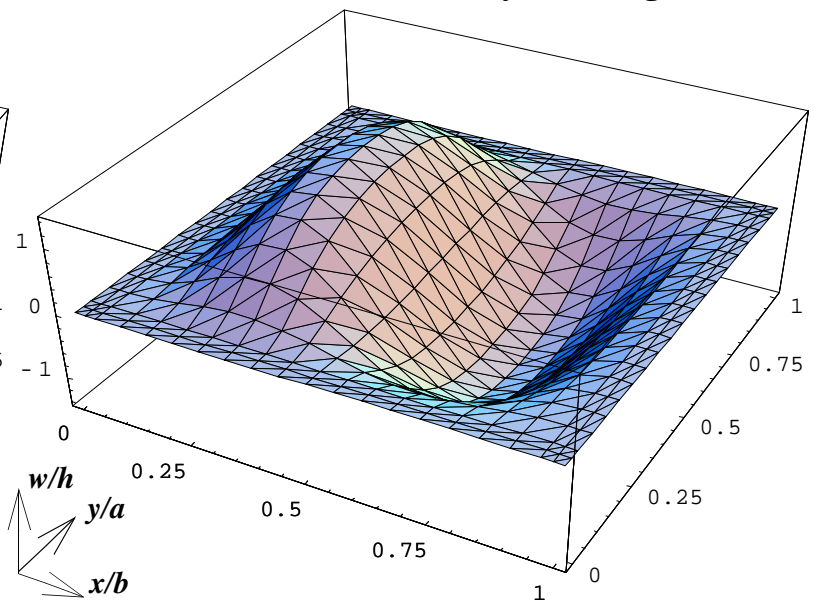

Fig. 3.9b: Configuration of Stiffened Plate after Secondary Buckling 
Since the restraint on $v$ at the simply supported edges for the case ${ }^{\mathrm{q}} \mathrm{P}_{1}$ is more severe than those for case ${ }^{\mathrm{q}} \mathrm{P}_{2}$, secondary buckling occurs at a lower level of end shortening in plate ${ }^{\mathrm{q}} \mathrm{P}_{1}$.

\subsubsection{Stiffened Quasi-isotropic Plates}

Figures 3.5 and 3.6 describe the response of stiffened quasi-isotropic perfect plates ${ }^{q} \mathrm{P}_{4},{ }^{\mathrm{q}} \mathrm{P}_{5},{ }^{\mathrm{q}} \mathrm{P}_{6}$, ${ }^{\mathrm{q}} \mathrm{P}_{7}$, and ${ }^{\mathrm{q}} \mathrm{P}_{8}$. The thickness of stiffeners in these plates correspond to $2.0,1.8,1.6,1.4$, and 1.2 $\mathrm{mm}$, respectively. In all these cases, no restraints on the $v$ displacement along the simply supported edges have been imposed while conducting the numerical simulation. As can be seen by comparing Figs. 3.3 and 3.5, case ${ }^{\mathrm{q}} \mathrm{P}_{8}$, which has the least stiff stiffeners, behaves much like case ${ }^{\mathrm{q}} \mathrm{P}_{3}$. The plate deflects out of plane in much the same manner and no secondary buckling is observed. As stiffer stiffeners are considered, secondary buckling becomes an issue. This, as seen from Figs. 3.5 and 3.6, is true of cases ${ }^{\mathrm{q}} \mathrm{P}_{4},{ }^{\mathrm{q}} \mathrm{P}_{5},{ }^{\mathrm{q}} \mathrm{P}_{6}$, and ${ }^{\mathrm{q}} \mathrm{P}_{7}$. In these cases the stiffness of the stiffener constrains the simply supported edge to remain relatively straight as compared to cases ${ }^{\mathrm{q}} \mathrm{P}_{3}$ and ${ }^{\mathrm{q}} \mathrm{P}_{8}$, where no secondary buckling is observed. This can be seen in Figs. 3.8a, 3.8b, and $3.8 \mathrm{c}$, which depict the variation of $v$ displacement along the simply supported edges of several plates. It is observed from these figures that plates which do not exhibit secondary buckling $\left({ }^{\mathrm{q}} \mathrm{P}_{3}\right.$ and ${ }^{\mathrm{q}} \mathrm{P}_{8}$ ) have significantly more deformed edges than those for plates which exhibit secondary buckling ( $\left({ }^{\mathrm{q}} \mathrm{P}_{1},{ }^{\mathrm{q}} \mathrm{P}_{2},{ }^{\mathrm{q}} \mathrm{P}_{4},{ }^{\mathrm{q}} \mathrm{P}_{5},{ }^{\mathrm{q}} \mathrm{P}_{6}\right.$, and ${ }^{\mathrm{q}} \mathrm{P}_{7}$ ), both prior to and after the secondary buckling event. Cases ${ }^{\mathrm{q}} \mathrm{P}_{5}$ and ${ }^{\mathrm{q}} \mathrm{P}_{7}$ are not depicted in Figs. 3.8b and 3.8c to avoid clutter. Among the unstiffened panels, this condition for a straight simply supported edge is exactly satisfied for plates ${ }^{\mathrm{q}} \mathrm{P}_{1}$ and ${ }^{\mathrm{q}} \mathrm{P}_{2}$. In the stiffened panel, stiffeners in panels ${ }^{\mathrm{q}} \mathrm{P}_{4},{ }^{\mathrm{q}} \mathrm{P}_{5},{ }^{\mathrm{q}} \mathrm{P}_{6}$, and ${ }^{\mathrm{q}} \mathrm{P}_{7}$ constrain the simply supported edges to remain "sufficiently straight," thereby triggering secondary buckling.

It should be noted that the $v$ displacement in Figs. $3.8 \mathrm{~b}$ and $3.8 \mathrm{c}$ are generally not symmetric with respect to the mid-length location along the edges. This effect would normally be associated with unbalanced plates, i.e., when the terms $A_{16}$ and $A_{26}$ in $A$ matrix are not zero. Here the effect is the result of $\partial w / \partial x$ and $\partial w / \partial y$ being in the expression for $\gamma_{x y}$ (see Eq. 5) and consequently $N_{x y}$ (see Eq. 10). Because of nonzero values of $D_{16}$ and $D_{26}, \partial w / \partial x$ and $\partial w / \partial y$ are not symmetric with respect to the centerlines of the plate. Therefore, there is an asymmetry to certain inplane responses, e.g., $v(x, b)$. To confirm this hypothesis, an equivalent case study was conducted where 
$D_{16}$ and $D_{26}$ were artificially set to zero and the finite-element analysis conducted. As expected, it was found that the response of $v(x, b)$ was symmetric with respect to the midlength location along the edge of the plate.

Comparing Figs. 3.4 and 3.6, it is seen that the loads associated with given levels of end shortening for the stiffened cases are much higher than those for the unstiffened cases. This is due to the influence of the stiffener, shortening the stiffeners and the plate requiring more force than simply shortening the plate. This is also seen in Fig. 3.7, which compares the loads borne by stiffeners with loads borne by the overall stiffened plates. It is seen in this figure that stiffeners bear approximately half of the total load borne by stiffened plate. As a result, referring to Figs. 3.4 and 3.6, when the stiffened plate shows secondary buckling, as in cases ${ }^{9} \mathrm{P}_{4},{ }^{9} \mathrm{P}_{5},{ }^{9} \mathrm{P}_{6}$, and ${ }^{9} \mathrm{P}_{7}$, the drop in load is not as dramatic as for the unstiffened cases that exhibit secondary buckling, namely ${ }^{\mathrm{q}} \mathrm{P}_{1}$ and ${ }^{\mathrm{q}} \mathrm{P}_{2}$.

Also, as seen from Figs. 3.4 and 3.6, the ordering of the onset of secondary buckling for the case of stiffened plates, with case $\mathrm{P}_{4}$ exhibiting secondary buckling at the lowest level of end shortening, is much like the ordering of the unstiffened cases, with case $\mathrm{P}_{1}$ exhibiting secondary buckling at the lowest level of end shortening. For both the unstiffened and stiffened plates, it is the cases with most restraint on the $v$ displacement at the simply supported edges that exhibit secondary buckling at the lowest level of end shortening.

Finally, stiffeners also affect the curvature of the plate near the simply supported edges of the plate. They offer considerable resistance to the rotation of the plate near the simply supported edges due to their torsional rigidity. It is seen from Figs. 3.2a and 3.2b that unstiffened panels are free to rotate out-of-plane just inside and just outside their simply supported edges. However, the portion of the stiffened plate just inside and just outside simply supported edges are forced to remain inplane, as seen from Figs. 3.9a and 3.9b. 


\subsection{INFLUENCE OF LAMINATION SEQUENCE}

This section addresses the sensitivity of secondary buckling of unstiffened clamped-simply supported plates to two classes of variations in the layup. The first class of these variations is assumed to be unintentional and due to manufacturing defects. Here, the influence of minor variations in the orientation of just one layer of the laminate is explored. These variations, as explained later, could be a source of out-of-plane imperfections in the plate. Three cases are considered in this section to address the role of unintentional variations on the response of the quasi-isotropic unstiffened plates, and three cases are considered to address the role of unintentional variations on the response of stiffened quasi-isotropic plates. The other class of variations in the layup is intentional and is simply a comparison of stacking arrangements, namely $[ \pm 45 / 0 / 90]_{2 s},\left[ \pm 45 / 0_{2}\right]_{2 s}$, and $\left[ \pm 45 / 90_{2}\right]_{2 \mathrm{~s}}$. These stacking arrangements are referred to, respectively, as quasi-isotropic, longitudinally stiff, and transversely stiff laminates. Nine case studies are considered in an effort to understand the influence of different stacking arrangements on the response of perfectly flat plates.

The imperfect plates have an intended lay-up of $[ \pm 45 / 0 / 90]_{2 s}$. For an imperfection, the top layer is assumed to have an offset of $5^{\circ}$ from the intended fiber orientation. Thus the top layer has an orientation of $40^{\circ}$ instead of $45^{\circ}$, as in the perfect plates. This offset provides the plate with a nonzero extension-bending coupling matrix, $\mathrm{B}$, and causes the plate to develop an out-of-plane deflection when the plate is cooled from the cure temperature by $-167^{\circ} \mathrm{C}$. Misaligned plies can be a very real source of imperfection in practice. Tables 3.4 and 3.5 list the cases studied to determine the role of lamination sequence and fiber misalignment imperfection, respectively.

\begin{tabular}{|c|c|c|c|}
\hline $\begin{array}{c}\text { Table 3.4: Cases Studied to Explore the Role of Lamination } \\
\text { Sequence on Postbuckling Response of Unstiffened Plates }\end{array}$ \\
\hline \hline & \multicolumn{3}{|c|}{ Plates without Imperfections } \\
\hline BC along SS edge & $v=0$ & $\begin{array}{c}\int \mathrm{N}_{y} d x=0 \\
v=\text { const. }\end{array}$ & $N_{y}=0$ \\
\hline$\left[ \pm 45 / 0_{2}\right]_{2 \mathrm{~s}}$ & ${ }^{0} \mathrm{P}_{1}$ & ${ }^{0} \mathrm{P}_{2}$ & ${ }^{0} \mathrm{P}_{3}$ \\
\hline$[ \pm 45 / 0 / 90]_{2 s}$ & ${ }^{\mathrm{q}} \mathrm{P}_{1}$ & ${ }^{\mathrm{q}} \mathrm{P}_{2}$ & ${ }^{\mathrm{q}} \mathrm{P}_{3}$ \\
\hline$\left[ \pm 45 / 90_{2}\right]_{2 \mathrm{~s}}$ & ${ }^{90} \mathrm{P}_{1}$ & ${ }^{90} \mathrm{P}_{2}$ & ${ }^{90} \mathrm{P}_{3}$ \\
\hline
\end{tabular}


Table 3.5: Cases Studied to Explore the Role of Imperfections on Postbuckling Response of Unstiffened and Stiffened Plates

\begin{tabular}{|c|c|c|c|c|c|c|}
\hline \hline & \multicolumn{3}{|c|}{ Unstiffened Plates } & \multicolumn{2}{c|}{ Stiffened Plates (thickness=t mm.) } \\
\hline BC along SS edge & $v=0$ & $\begin{array}{c}\int \mathrm{N}_{y} d x=0 \\
v=\text { const. }\end{array}$ & $N_{y}=0$ & 2.0 & 1.6 & 1.2 \\
\hline$[ \pm 45 / 0 / 90]_{2 \mathrm{~s}}$ & ${ }^{\mathrm{q}} \mathrm{I}_{1}$ & ${ }^{\mathrm{q}} \mathrm{I}_{2}$ & ${ }^{\mathrm{q}} \mathrm{I}_{3}$ & ${ }^{\mathrm{q}} \mathrm{I}_{4}$ & ${ }^{\mathrm{q}} \mathrm{I}_{6}$ & ${ }^{\mathrm{q}} \mathrm{I}_{8}$ \\
\hline
\end{tabular}

\subsubsection{Role of Lamination Sequence on the Response of Unstiffened Panels}

Figures 3.10, 3.12, and 3.14 depict the influence of plate layup on the out-of-plane deflection response of plates subjected to a varying set of boundary conditions, as listed in Table 3.4. Figures $3.11,3.13$, and 3.15 highlight the influence of lamination sequence on the end shortening response of panels subjected to a varying set of boundary conditions.

Prebuckling inplane stiffness in the loading direction is maximum for the longitudinally stiff plates $\left({ }^{0} \mathrm{P}_{1},{ }^{0} \mathrm{P}_{2},{ }^{0} \mathrm{P}_{3}\right)$, and is least for transversely stiff plates $\left({ }^{90} \mathrm{P}_{1},{ }^{90} \mathrm{P}_{2},{ }^{90} \mathrm{P}_{3}\right)$. This is borne out by the slopes of the relations shown in Figs. 3.11, 3.13, and 3.15. We also learn from these figures that the longitudinally stiff plates take the highest load to reach the buckling point, while the transversely stiff plates take the least load. All the plates initially buckle to assume the shape as shown in Fig. 3.2a. Further, Figs. 3.11, 3.13, and 3.15 also show that for a given set of boundary conditions, the longitudinally stiff plate has to be subjected to the least inplane compressive end displacement to make it buckle, while the transversely stiff plate needs the maximum inplane end displacement to achieve the same end. The high inplane stiffness of the longitudinally stiff plate is the responsible for this behavior of these plates.

The postbuckling stiffnesses of plates in Table 3.4, computed as $\partial P / \partial \Delta$, once again follows the same trends as those exhibited by their respective prebuckling stiffnesses. As expected, the longitudinally stiff, quasi-isotropic, and transversely stiff plates have the highest, intermediate, and the least postbuckling stiffnesses. Very much similar to the discussions in section on 3.3, it is seen that all plates, irrespective of their layup, do not exhibit secondary buckling if the $v$ displacement along the simply supported edges is not constrained. This is borne out by Figs. 3.14 and 3.15. In 
all other cases, however, secondary buckling does occur. Figures 3.11 and 3.13 indicate that the stiffening of the laminate in the longitudinal direction has a pronounced effect on the secondary buckling of the laminate. This stiffening has four effects:

- Stiffening causes an increase in the inplane compressive load corresponding to the secondary buckling point. This increase is larger than the increase in respective buckling loads. Since plates ${ }^{0} \mathrm{P}_{1}$ and ${ }^{0} \mathrm{P}_{2}$ are stiffest in the loading direction, they have the highest secondary buckling loads. For a similar reason, secondary buckling loads are the least for plates ${ }^{90} \mathrm{P}_{1}$ and ${ }^{90} \mathrm{P}_{2}$.

- Stiffening causes an increase in the inplane compressive displacement corresponding to the secondary buckling point. Once again, ${ }^{0} \mathrm{P}_{1}$ and ${ }^{0} \mathrm{P}_{2}$ need the maximum and ${ }^{90} \mathrm{P}_{1}$ and ${ }^{90} \mathrm{P}_{2}$ need the least amounts of end shortening to trigger secondary buckling.

- The pre-secondary buckling out-of-plane configuration for all the plates is essentially same as that shown in Fig. 3.2a. However, at the point of secondary buckling, the amplitude of this configuration is maximum, intermediate, and least for the longitudinally stiff, quasi-isotropic, and transversely stiff plates, respectively. This trend is evident from Figs. 3.10 and 3.12.

- The occurrence of secondary buckling is accompanied by a drop in the external load carried by the plate. The magnitude of this drop is maximum, intermediate, and least for the longitudinally stiff, quasi-isotropic, and transversely stiff plates, respectively. This can be seen from Figs. 3.11 and 3.13.

\subsubsection{Role of Imperfections on the Response of Quasi-isotropic Panels}

Figures 3.16 through 3.20 provide information on the influence of imperfections on the postbuckling response of the unstiffened plates. The source of these imperfections, as mentioned earlier, is a misaligned outer ply. Calculations reveal that the magnitude of the out-of-plane deflection from this form of imperfection is on the order of $1 \%$ of the plate thickness. This can be seen in Figs. 3.16a and 3.16b. These figures are depictions of out-of-plane deflections of unstiffened and stiff- 
ened plates owing their existence to misaligned top ply and a cooling from curing. Please note that the vertical axes corresponding to the magnitude of imperfections have different scales for the unstiffened and stiffened plates. This has been done to draw the attention of the reader to the overall shape of the imperfections. During the course of subsequent numerical investigations, it was found that these imperfections, despite their such low magnitudes, have an influence on the response of the plate. 


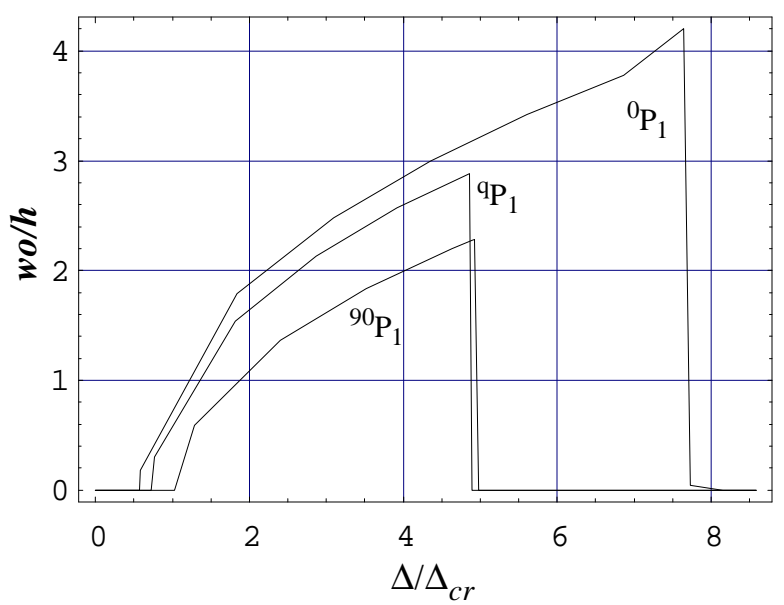

Fig. 3.10: Deflection Response of Perfect Plates with Fixed SS Edges

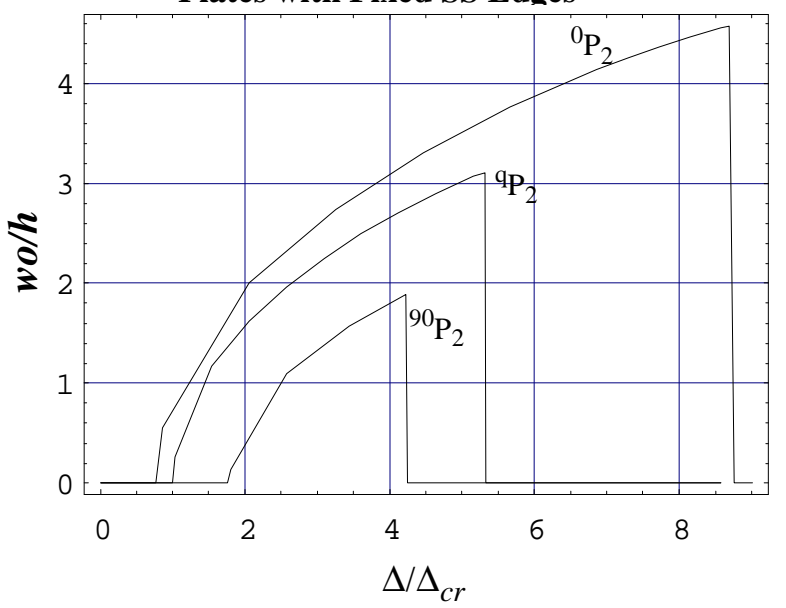

Fig. 3.12: Deflection Response of Perfect Plates with Straight SS Edges

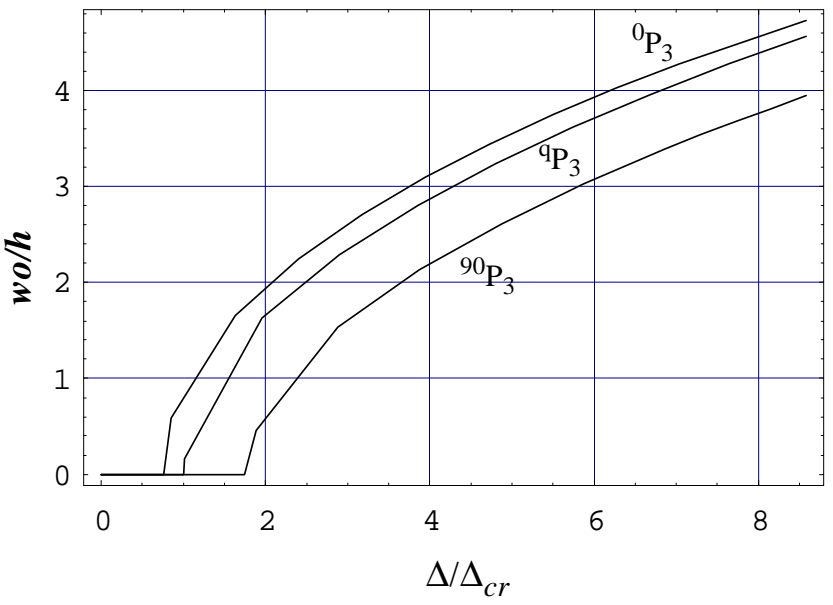

Fig. 3.14: Deflection Response of Perfect Plates with Unconstrained SS Edges

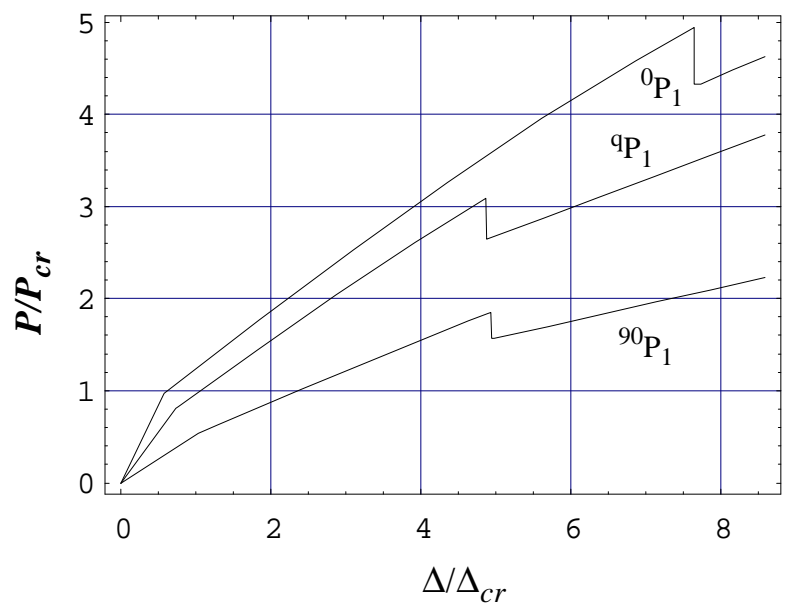

Fig. 3.11: Load Response of Perfect Plates with Fixed SS Edges

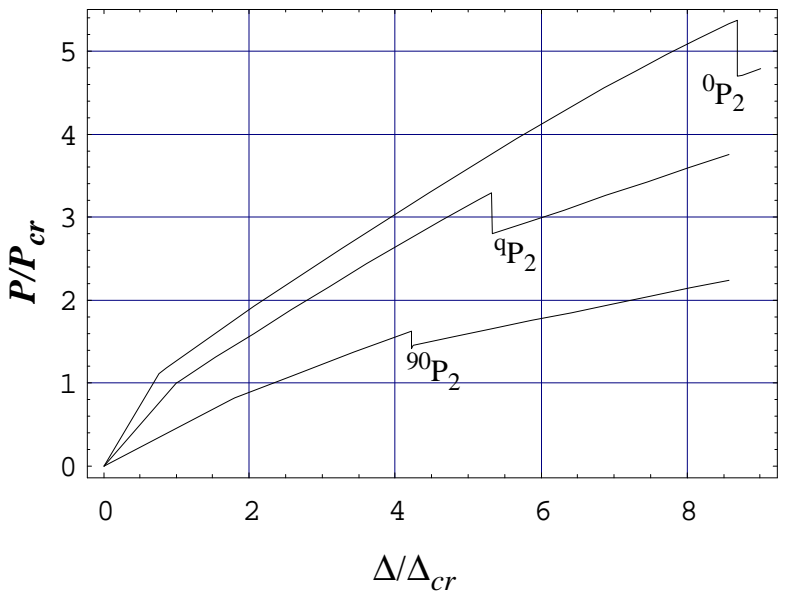

Fig. 3.13: Load Response of Perfect Plates with Straight SS Edges

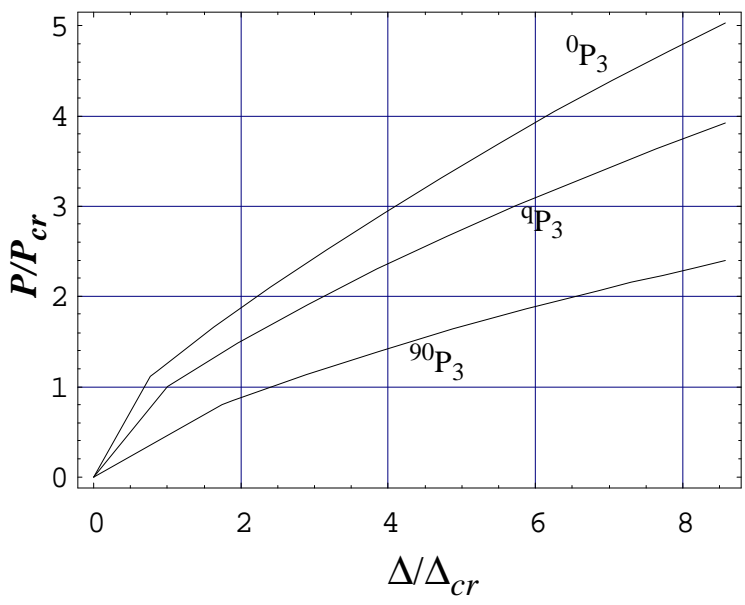

Fig. 3.15: Load Response of Perfect Plates with Unconstrained SS Edges 


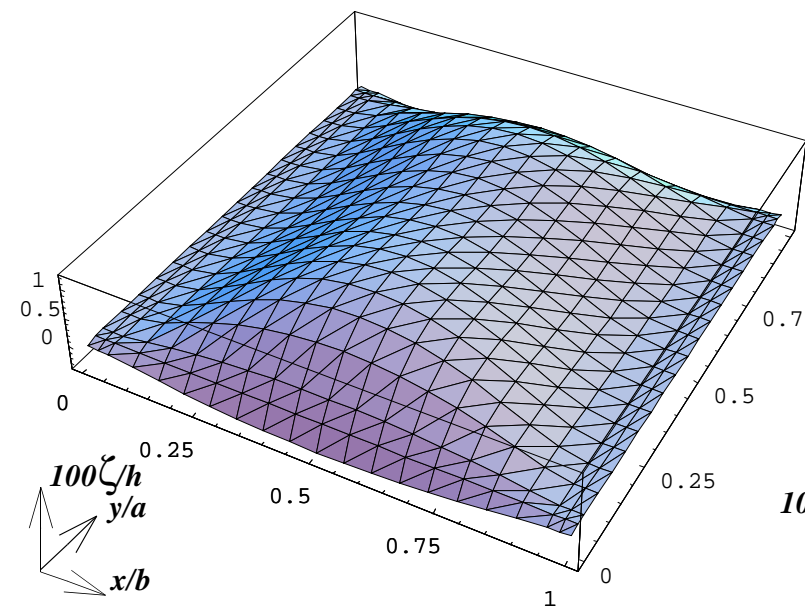

Fig. 3.16a: Imperfections in Unstiffened Quasi-isotropic Plate

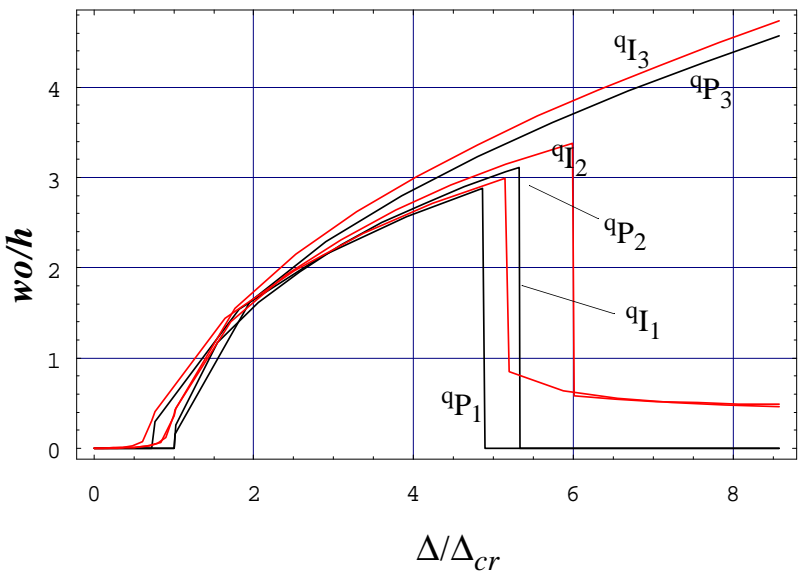

Fig. 3.17: Deflection Response of Imperfect Quasi-isotropic Unstiffened Plates

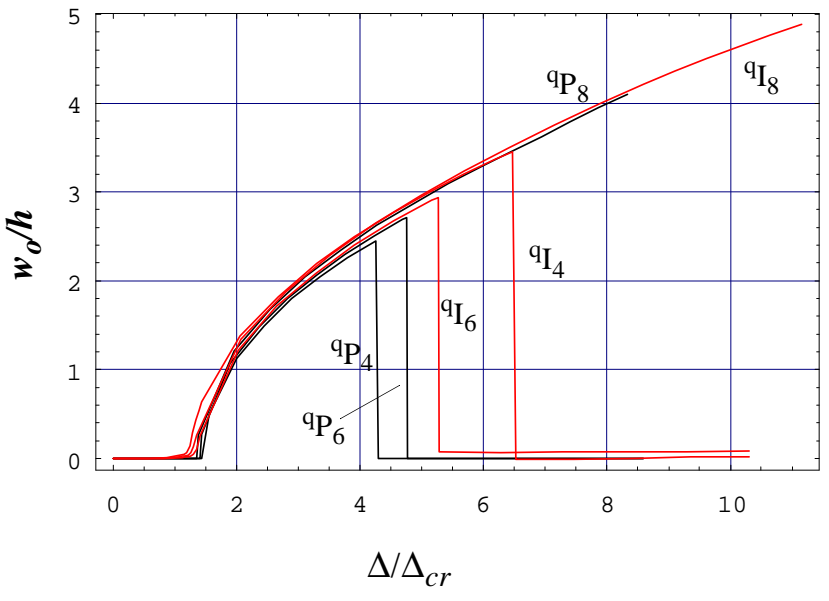

Fig. 3.19: Deflection Response of Imperfect Quasi-isotropic Stiffened Plates

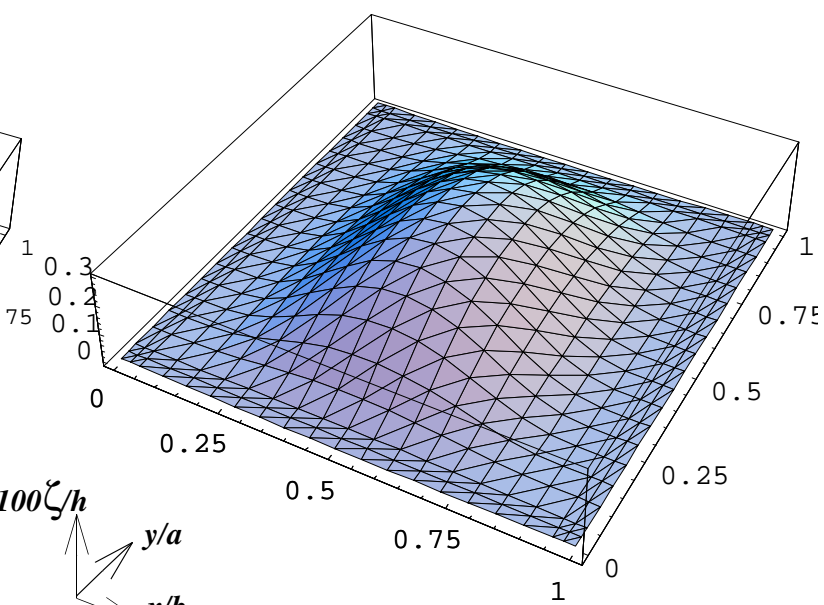

$x / b$ Fig. 3.16b: Imperfections in Stiffened Quasi-isotropic Plate $(t=2.0 \mathrm{~mm})$

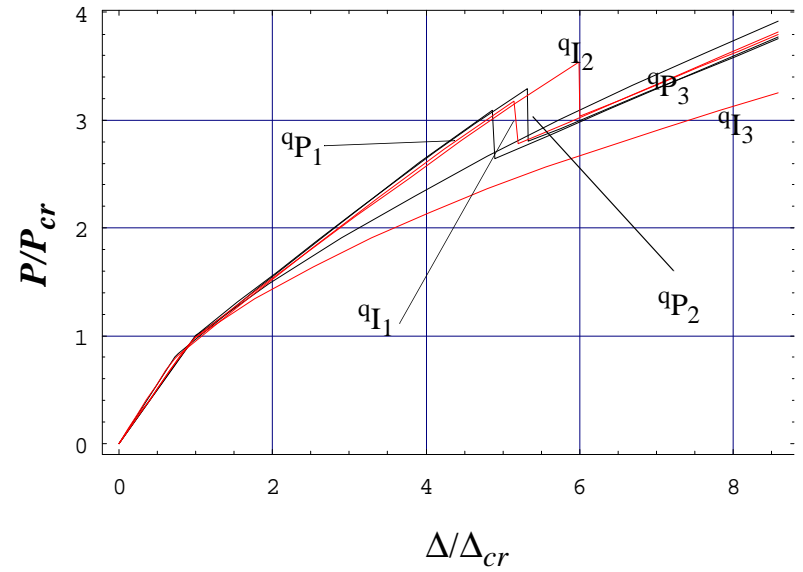

Fig. 3.18: Load Response of Imperfect Quasi-isotropic Unstiffened Plates

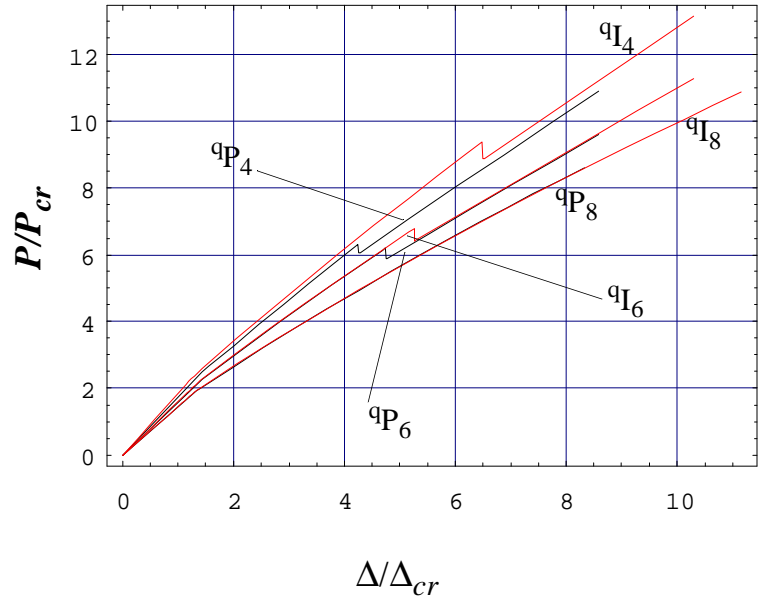

Fig. 3.20: Load Response of Imperfect Quasi-isotropic Stiffened Plates 


\subsubsection{Unstiffened Plates}

From Figs. 3.17 and 3.18 the following observation can be made:

- Due to imperfections, the plates start deflecting out of plane from the very start of the end shortening process. Consequently, buckling of the plate does not occur. This can be seen in Fig. 3.17.

- A comparison of the responses of cases ${ }^{\mathrm{q}} \mathrm{I}_{1},{ }^{\mathrm{q}} \mathrm{I}_{2},{ }^{\mathrm{q}} \mathrm{I}_{3}$ with respect to those for cases ${ }^{9} \mathrm{P}_{1},{ }^{\mathrm{q}} \mathrm{P}_{2},{ }^{\mathrm{q}} \mathrm{P}_{3}$, Fig. 3.17, reveals that for the same level of end shortening, imperfect plates tend to deflect out of plane more than their perfect counterparts.

- The most noticeable impact of imperfections on the postbuckling response of the plate is on the onset of the secondary buckling event. Both Figs. 3.17 and 3.18 show that secondary buckling in imperfect plates occurs at higher values of end shortening than their perfect counterparts. From this it can be concluded that even small imperfections can somewhat stabilize the postbuckled configuration of the plate.

- Another effect of these imperfections is that the shape of the plate after the occurrence of secondary buckling is different than its analog perfect plate. While in the case of perfect plates the deflection of plate's center drops to zero, it does not do so in the case of imperfect plates. This is felt to be due to the existence of the $B$ matrix, which produces out-of-plane deflections in the presence of end shortening, even for the linear problem.

\subsubsection{Stiffened Plates}

Figures 3.19 and 3.20 show the influence of the imperfection on the response of the stiffened plates. The role played by imperfections in stiffened cases is similar to their role in unstiffened plates, namely, the onset of secondary buckling occurs at a higher level of end shortening when imperfections are present. Also, it appears that the difference in levels of end shortening between the imperfect and perfect cases is larger for the stiffened plates than for the unstiffened plates. For example, the difference in end shortening values at secondary buckling between cases ${ }^{9} I_{4}$ and ${ }^{9} P_{4}$ 
in Fig. 3.19 is higher than the difference between cases ${ }^{\mathrm{q}} \mathrm{I}_{1}$ and ${ }^{\mathrm{q}} \mathrm{P}_{1}$ in Fig. 3.17. The same can be said of the difference between cases ${ }^{\mathrm{q}} \mathrm{I}_{6}$ and ${ }^{\mathrm{q}} \mathrm{P}_{6}$ in Fig. 3.19 and ${ }^{\mathrm{q}} \mathrm{I}_{2}$ and ${ }^{\mathrm{q}} \mathrm{P}_{2}$ in Fig. 3.17. This is felt to be due to the fact that the stiffeners participate somewhat in the out-of-plane postbuckling response. Though they are confined to the simply supported edges, they are of finite width. The width of the stiffener that is inside the line of simple supports, $d / 2$, must deflect somewhat out of plane as the plate buckles. This small portion of the stiffener which must deflect out of plane does add to the overall bending resistance. Another observation regarding the stiffened perfect and stiffened imperfect cases can be seen in Fig. 3.19. For example, it is seen that the responses for cases ${ }^{\mathrm{q}} \mathrm{P}_{8}$ and ${ }^{\mathrm{q}} \mathrm{I}_{8}$ practically coincide, the imperfection not playing an important role. This is in contrast to the responses for cases ${ }^{\mathrm{q}} \mathrm{P}_{3}$ and ${ }^{\mathrm{q}} \mathrm{I}_{3}$ in Fig. 3.17.

\subsection{INTERACTION OF LAMINATION SEQUENCE AND STIFFENER THICKNESS}

Figures 3.21 through 3.30 highlight the interaction of lamination sequence and stiffener thickness and their cumulative influence on the postbuckling response of composite panels subjected to compressive loads. These figures are based on the fifteen case studies shown in Table 3.5. This table is a repeat of a portion of Table 3.1.

\begin{tabular}{|c|c|c|c|c|c|}
\hline \multicolumn{6}{|c|}{$\begin{array}{l}\text { Table 3.5: Case Studies Conducted to Understand the Interaction between Layup and } \\
\text { Stiffener Thickness and their Influence on Postbuckling }\end{array}$} \\
\hline \multirow[b]{2}{*}{ Layup } & \multicolumn{5}{|c|}{ Thickness of stiffeners $t \mathrm{~mm}$} \\
\hline & 2.0 & 1.8 & 1.6 & 1.4 & 1.2 \\
\hline$\left[ \pm 45 / 0_{2}\right]_{2 s}$ & ${ }^{0} \mathrm{P}_{4}$ & ${ }^{0} \mathrm{P}_{5}$ & ${ }^{0} \mathrm{P}_{6}$ & ${ }^{0} \mathrm{P}_{7}$ & ${ }^{0} \mathrm{P}_{8}$ \\
\hline$[ \pm 45 / 0 / 90]_{2 \mathrm{~s}}$ & ${ }^{\mathrm{q}} \mathrm{P}_{4}$ & ${ }^{\mathrm{q}} \mathrm{P}_{5}$ & ${ }^{\mathrm{q}} \mathrm{P}_{6}$ & ${ }^{\mathrm{q}} \mathrm{P}_{7}$ & ${ }^{\mathrm{q}} \mathrm{P}_{8}$ \\
\hline$\left[ \pm 45 / 90_{2}\right]_{2 \mathrm{~s}}$ & ${ }^{90} \mathrm{P}_{4}$ & ${ }^{90} \mathrm{P}_{5}$ & ${ }^{90} \mathrm{P}_{6}$ & ${ }^{90} \mathrm{P}_{7}$ & ${ }^{90} \mathrm{P}_{8}$ \\
\hline
\end{tabular}

Like the previous format, Figs. 3.21, 3.23, 3.25, 3.27, and 3.29 show the relationship between the normalized applied end shortening and the normalized out-of-plane deflections. Figures 3.22, $3.24,3.26,3.28$, and 3.30 are normalized plots of load versus normalized end shortening. In all these figures results from the classic case ${ }^{{ }^{9}} P_{3}$ have also been shown for comparison purposes. Based on the observations of these figures, the following conclusions could be drawn. 


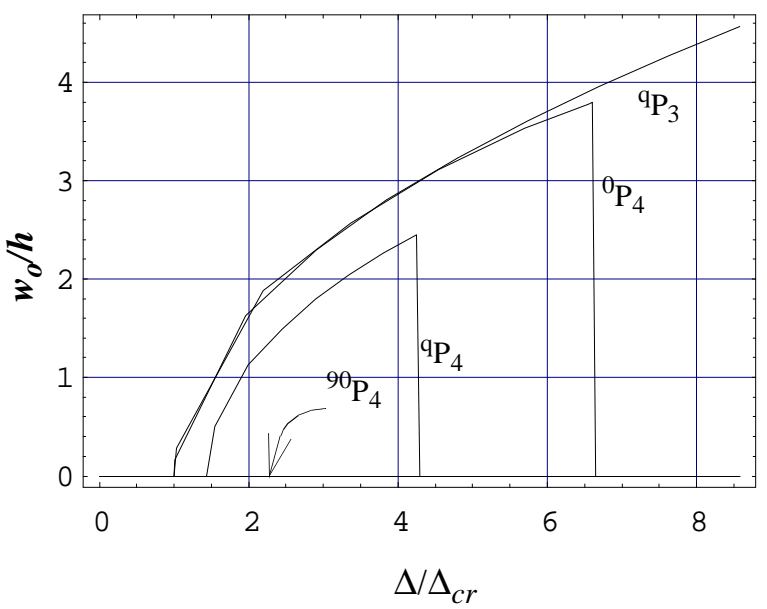

Fig. 3.21: Deflection Response of Perfectly

Flat Stiffened Plates $(t=2.0 \mathrm{~mm})$

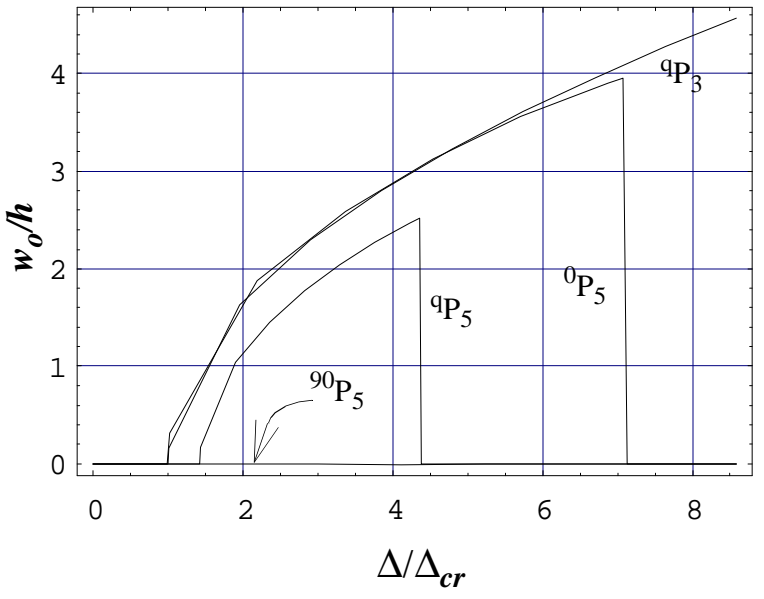

Fig. 3.23: Deflection Response of Perfectly Flat Stiffened Plates $(t=1.8 \mathrm{~mm})$

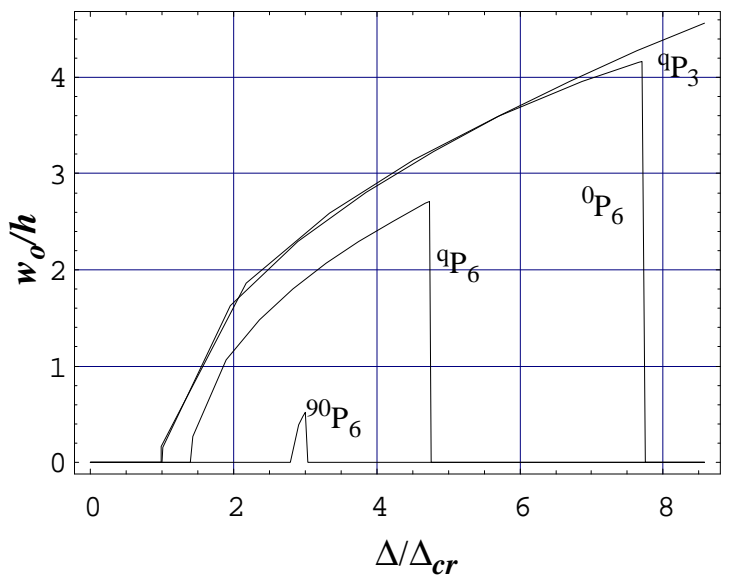

Fig. 3.25: Deflection Response of Perfectly Flat Stiffened Plates $(t=1.6 \mathrm{~mm})$

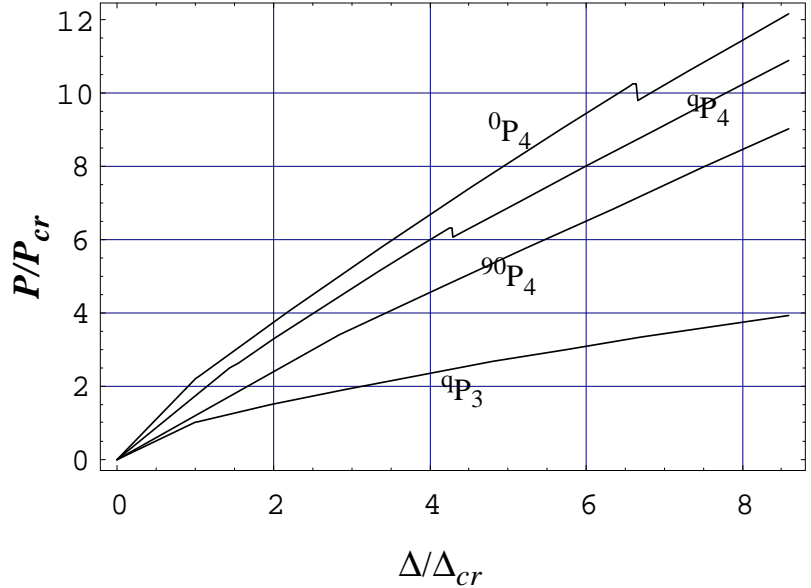

Fig. 3.22: Load Response of Perfectly Flat Stiffened Plates $(t=2.0 \mathrm{~mm})$

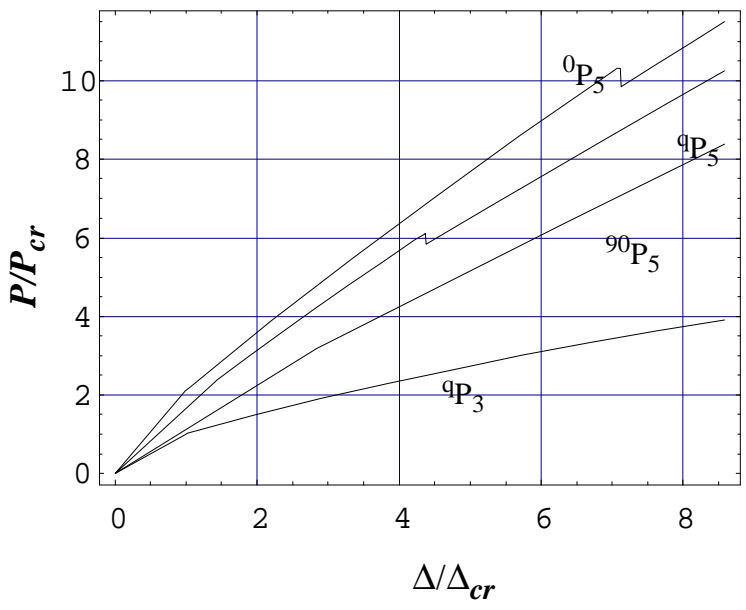

Fig. 3.24: Load Response of Perfectly Flat Stiffened Plates $(t=1.8 \mathrm{~mm})$

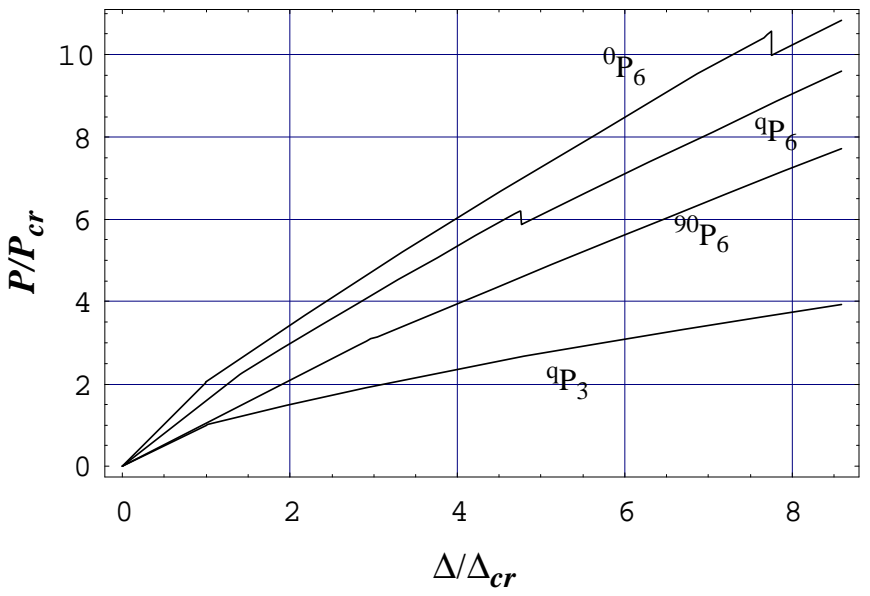

Fig. 3.26: Load Response of Perfectly Flat Stiffened Plates $(t=1.6 \mathrm{~mm})$ 


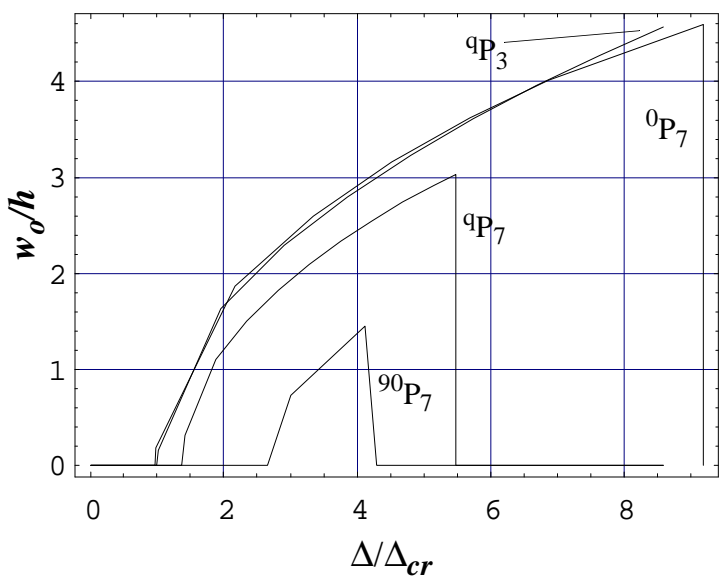

Fig. 3.27: Deflection Response of Perfectly Flat Stiffened Plates $(t=1.4 \mathrm{~mm})$

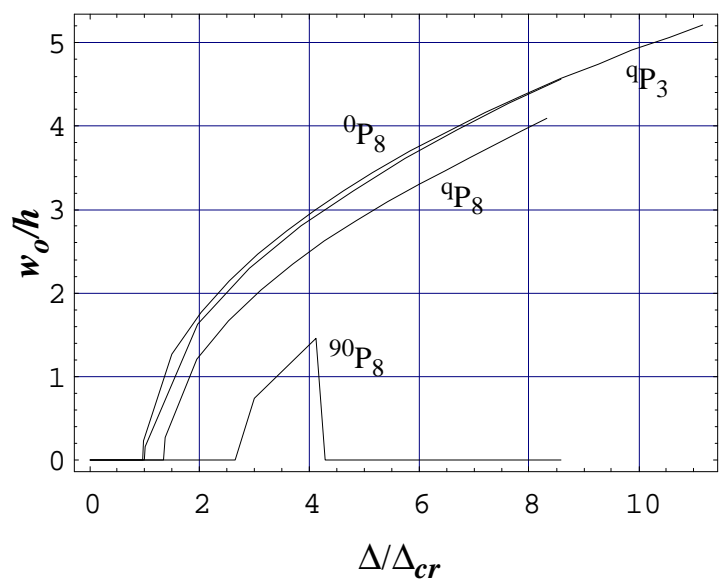

Fig. 3.29: Deflection Response of Perfectly Flat Stiffened Plates $(t=1.2 \mathrm{~mm})$

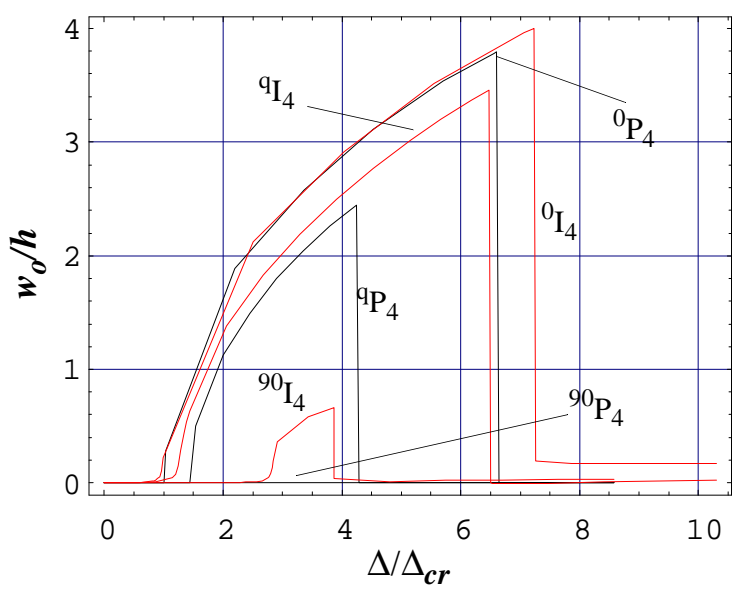

Fig. 3.31: Deflection Response of Imperfect and Stiffened Plates $(t=2.0 \mathrm{~mm})$

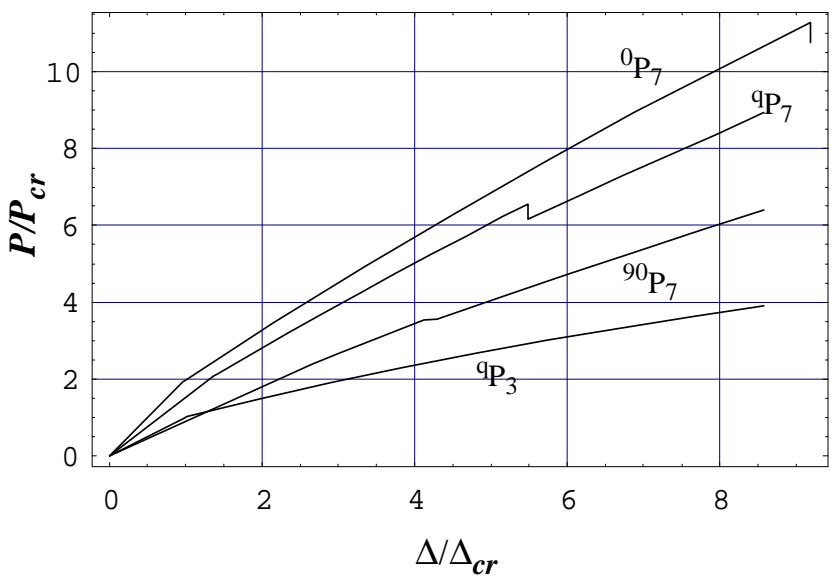

Fig. 3.28: Load Response of Perfectly Flat Stiffened Plates $(t=1.4 \mathrm{~mm})$

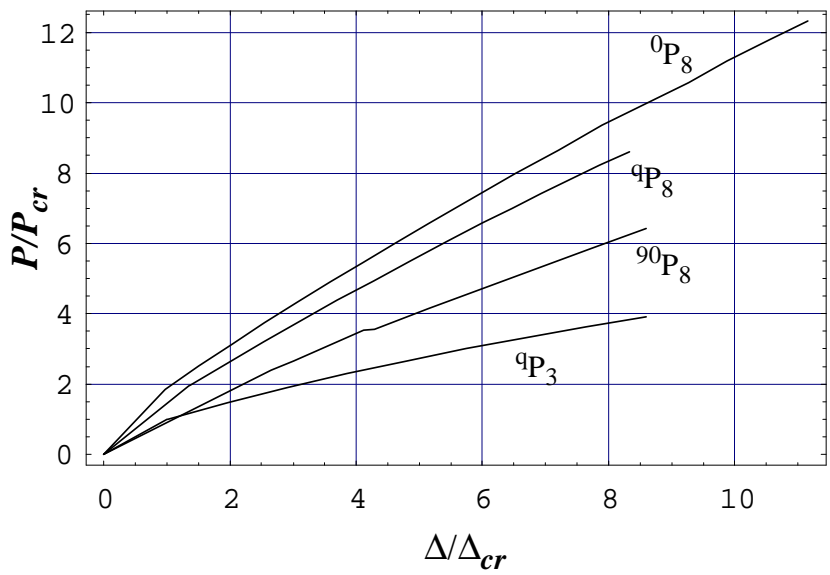

Fig. 3.30: Load Response of Perfectly Flat Stiffened Plates $(t=1.2 \mathrm{~mm})$

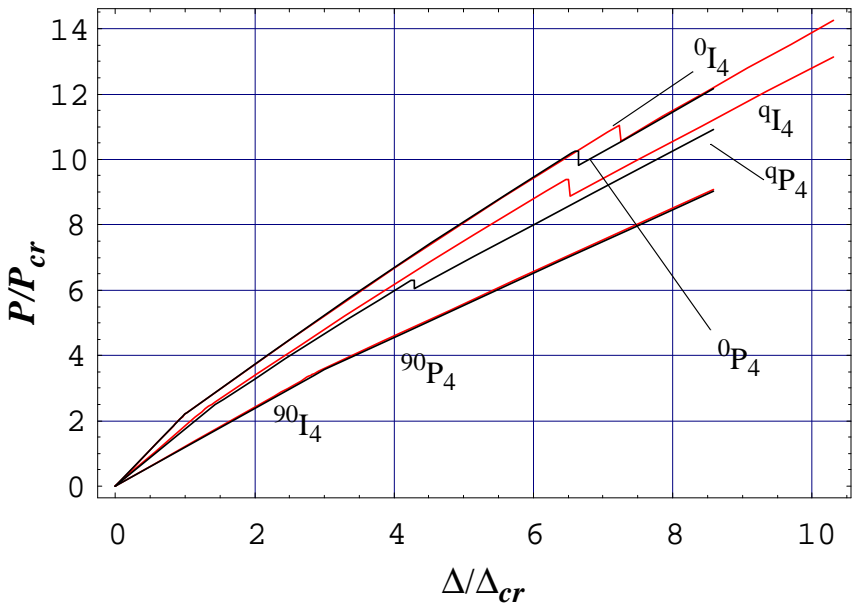

Fig. 3.32: Load Response of Imperfect and Stiffened Plates $(t=2.0 \mathrm{~mm})$ 
- For a specified value of stiffener thickness, the prebuckling stiffness of the plate is highest for the longitudinally stiff plate and least for the transversely stiff plate. In the prebuckling range stiffeners merely enhance the overall inplane stiffness of the plate.

- As seen in the deflection response curves, the value of uniform end shortening $\Delta / \Delta_{c r}$ required to initiate primary buckling is least for the longitudinally stiff plate. A decrease in plate's inplane stiffness in the loading direction results in an increase in the value of end shortening corresponding to the primary buckling event. This can be seen in Figs. 3.25, 3.27, and 3.29. It is also seen through these figures that for a given lamination sequence, the end shortening value corresponding to primary buckling is not very sensitive to stiffener thickness. This could be attributed to the fact that the stiffeners cannot bend out of plane due to simply supported conditions, thereby they contribute little to the out-of-plane characteristics of the plate at low loads. In contrast to this, the buckling loads for a plate appear to drop with reductions in stiffener thicknesses. This is a direct consequence of the fact that a heavier stiffener result in higher inplane plate stiffness.

- The out-of-plane deflection responses for two transversely stiff plates $\left({ }^{90} \mathrm{P}_{4}\right.$ and $\left.{ }^{90} \mathrm{P}_{5}\right)$ are flat. This is seen in Figs. 3.21 and 3.23. This is so since these plates directly buckle into a pattern which has two half-waves in the loading direction. The thickness of the stiffeners for these plates are 2.0 and $1.8 \mathrm{~mm}$ respectively. However, plates ${ }^{90} \mathrm{P}_{6},{ }^{90} \mathrm{P}_{7}$, and ${ }^{90} \mathrm{P}_{8}$ have primary buckling configurations with one half-wave in the loading as well as transverse directions. This can be seen in Figs. 3.25, 3.27, and 3.29. We also observe that all other plates' primary buckling configurations have one half-wave in loading as well as transverse directions.

- From Figs. 3.22, 3.24, 3.26, 3.28, and 3.30, it is seen that the drop in load associated with secondary buckling of plates decreases with decreasing inplane stiffness of the plate in the loading direction. Since a transversely stiff plate is softest in the loading direction, there is very little load drop associated with it's corresponding secondary buckling event. Further, the corresponding load drops for plates with longitudinally stiff laminate are considerably the highest. It must be pointed out that secondary buckling load drops for ${ }^{90} \mathrm{P}_{4}$ and ${ }^{90} \mathrm{P}_{5}$ plates with stiffener thicknesses of 2.0 and $1.8 \mathrm{~mm}$, respectively, is a non-issue. This is so since they directly buckle into a shape with two half-waves in the loading direction. For all other plates however, secondary load drop intensities are driven by the 
inplane stiffness of the plate in the loading direction, as discussed earlier.

- From Figs. 3.21, 3.23, 3.25, 3.27, and 3.29, it is seen that the end shortening displacement associated with secondary buckling of plates decreases with decreasing inplane stiffness of the plate in the loading direction. Once again, it must be pointed out that this observation for ${ }^{90} \mathrm{P}_{4}$ and ${ }^{90} \mathrm{P}_{5}$ plates with stiffener thicknesses of 2.0 and $1.8 \mathrm{~mm}$, respectively, is a non-issue since they directly buckle into a shape with two half-waves in the loading direction. For all other plates, however, end shortening displacements associated with secondary buckling event are apparently driven by the inplane stiffness of the plate in the loading direction.

Figs. 3.31 and 3.32 compare the response of stiffened perfect plates with their imperfect analogs. The stiffness thickness in this case is $2.0 \mathrm{~mm}$. It is seen from these figures that, very similar to unstiffened plates, imperfections delay the secondary buckling event by significant amounts in terms of end shortening as well as inplane compressive load. Figure 3.32 also shows that this increase in load and end displacement for the secondary buckling event tends to increase with decreasing inplane stiffness of the plate in the loading direction. It is also seen that imperfections tend to bias the plate's primary buckling mode more towards a shape which has one half-wave in the loading and transverse directions. This is seen by comparing the responses of perfect plate ${ }^{90} \mathrm{P}_{4}$ with its imperfect analog ${ }^{90} \mathrm{I}_{4}$. As seen in Fig. 3.21, the primary buckling mode of the perfect plate $\left({ }^{90} \mathrm{P}_{4}\right)$ has two half-waves and one half-wave in loading and transverse directions, respectively. However, the presence of imperfections in a similar plate $\left({ }^{90} \mathrm{I}_{4}\right)$ biases the plate to buckle initially into a pattern which has one half-wave in loading as well as transverse directions. This is also seen in Fig. 3.31.

\subsection{METALLIC PLATES}

Figures 3.33 and 3.34 compare the secondary buckling responses of metallic plates ${ }^{\mathrm{m}} \mathrm{P}_{1},{ }^{\mathrm{m}} \mathrm{P}_{2}$, and ${ }^{m} \mathrm{P}_{3}$ with those of perfectly flat unstiffened quasi-isotropic plates. The metal considered in these cases is aluminum. Figures 3.33 and 3.34 correspond to the out-of-plane deflection responses and load responses, respectively, of the metallic and quasi-isotropic plates. It is seen from these figures that, in a qualitative sense, the nature of response curves, both for out-of-plane deflection and 
load, for metallic plates is similar to the quasi-isotropic counterparts. The difference in response is merely quantitative in nature. The metallic plates considered here, due to their higher inplane as well as bending stiffnesses, tend undergo buckling and secondary buckling at higher loads.

\subsection{INFLUENCE OF OUT-OF-PLANE SHEAR DEFORMATIONS ON SECONDARY BUCKLING}

In all, six plates were analyzed to examine the influences of out-of-plane shear deformations on the secondary buckling characteristics of composite plates. All the properties of these plates, except for out-of-plane shear moduli $G_{31}$ and $G_{23}$, were identical to those of the unstiffened quasi-isotropic plate. Among these six plates, three of them namely, ${ }^{10} \mathrm{~S}_{1},{ }^{10} \mathrm{~S}_{2}$, and ${ }^{10} \mathrm{~S}_{3}$, were assigned a value of $10 G_{12}$ for the out-of-plane shear moduli $G_{23}$ and $G_{31}$. For the remaining three plates, namely ${ }^{100} \mathrm{~S}_{1},{ }^{100} \mathrm{~S}_{2}$, and ${ }^{100} \mathrm{~S}_{3}$, the out-of-plane shear moduli were fixed at $100 G_{12}$. The intent behind these computations was to explore how a plate which was stiffer in out-of-plane shear, when subjected to inplane compression, would behave relative to its normal-stiffness counterpart. Prior to this study it was surmised that the role of out-of-plane shear, if any, would be minimal. This study of the influence of $G_{13}$ and $G_{23}$ was meant to confirm or reject this assumption.

Figures 3.35 through 3.40 provide an understanding the influences of out-of-plane shear deformations on the postbuckling characteristics of unstiffened quasi-isotropic plates. Figures 3.35 and

3.36 depict the comparative out-of-plane deflection and load responses of plates ${ }^{10} \mathrm{~S}_{\beta}$ and ${ }^{100} \mathrm{~S}_{\beta}$ relative to their normal counterparts ${ }^{\mathrm{q}} \mathrm{P}_{\beta}$, where the subscript $\beta$ could assume values of 1,2 , and 3 . It is seen from these figures that plates which have high values of out-of-plane shear stiffness behave almost identically to the plates with normal values of $G_{13}$ and $G_{23}$. This is possible only if magnitudes of out-of-plane shear strains, $\gamma_{z x}$ and $\gamma_{y z}$, are inconsequential. The definitions of these shear strains, given in Eqs. (5), are reproduced here for convenience as 


$$
\begin{aligned}
& \gamma_{x z}^{0}=\psi_{x}^{o}+\frac{\partial w}{\partial x} \\
& \gamma_{y z}^{0}=\psi_{y}^{o}+\frac{\partial w}{\partial y}
\end{aligned}
$$

From these definitions, it is clear that $\gamma_{z x}^{0}$ will be exactly zero if the ratio of it's components, $\psi^{o}{ }_{x}$ and $\partial w / \partial x$, is -1. A similar mathematical condition can also be stated for shear strain $\gamma_{y z}^{0}$. Figure 3.37 shows the variation of the individual components of $\gamma_{z x}^{0}$, i.e., $\partial w / \partial x$ and $\psi^{o}{ }_{x}$, along the midaxis $y=b / 2$ of plates ${ }^{100} \mathrm{~S}_{1},{ }^{10} \mathrm{~S}_{1}$, and ${ }^{\mathrm{q}} \mathrm{P}_{1}$. It is seen in the figure that the condition for the vanishing of $\gamma_{z x}^{0}$, as outlined above, is approximately satisfied for all the three plates. This is also confirmed from Fig. 3.38 which shows the variation of $\gamma_{z x}^{0}$ along the mid-axis of the plates ${ }^{100} \mathrm{~S}_{1}$, ${ }^{10} \mathrm{~S}_{1}$, and ${ }^{\mathrm{q}} \mathrm{P}_{1}$. The presence of a small amount of non-zero shear strain, $\gamma_{z x}^{\mathrm{o}}$, as shown in Fig. 3.38 can be attributed to the fact that numerical solutions tend to converge slowly with respect to the spatial derivatives of field variables, e.g., out-of-plane deflection, $w$. In fact, since the normal through-thickness shear modulus, the times 10 through-thickness shear modulus, and the times 100 through-thickness shear modulus, have very similar shear strain magnitudes for $\gamma_{z x}^{0}$, it can be concluded that the shear strain levels for $\gamma_{z x}^{0}$ in these postbuckling problems are at the same level as the error in taking derivatives of the deformations to find the strains, and therefore are not important. With the help of Figs. 3.39 and 3.40, a similar conclusion can also be made about shear strain $\gamma_{y z}^{0}$. On the basis of the preceding discussion, it can be reasonably concluded that out-ofplane shear strains have negligible impact on the secondary buckling characteristics of the quasiisotropic unstiffened plates.

\subsection{CLOSURE}

In this chapter, results of numerical studies conducted on the response of clamped/simply supported plates subjected to inplane compressive loads have been presented and analyzed. The issue of sensitivity of these results to variations in lamination sequence, presence of imperfections, variations in boundary conditions, and stiffener thickness have been explored and discussed. The next chapter gives the details of the experimental setup used to corroborate some of these results and conclusions by comparing experimental results to finite element predictions. 


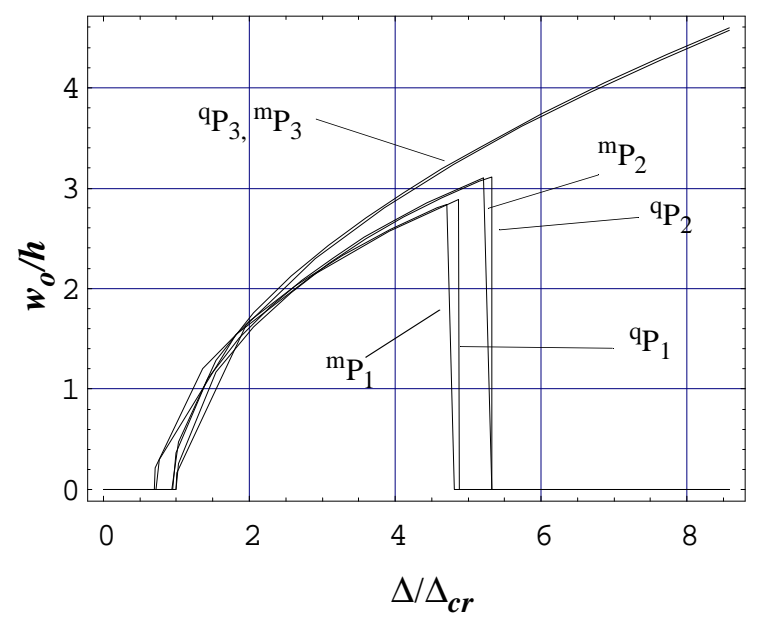

Fig. 3.33: Deflection Response of Unstiffened Quasi-isotropic and Metallic Plates

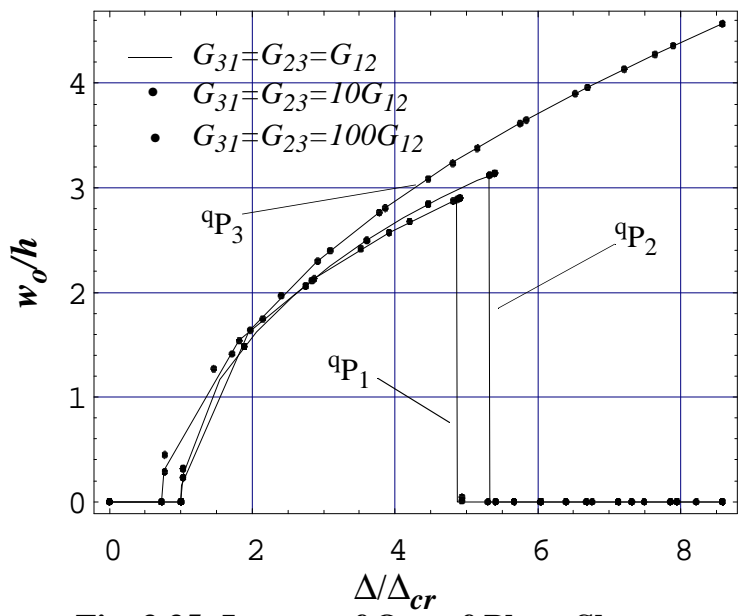

Fig. 3.35: Impact of Out-of-Plane Shear Stiffness on Deflection Response

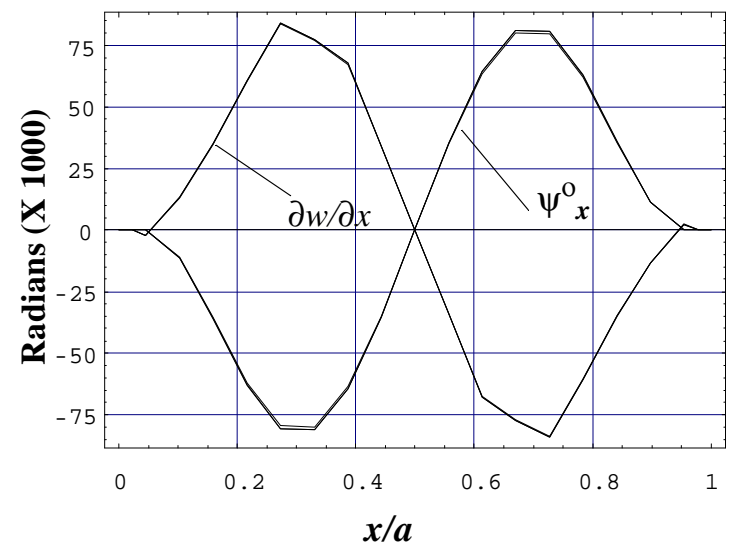

Fig. 3.37: Variation of $\psi^{0}{ }_{x}$ and $\partial w / \partial x$ along Vertical Mid-axis of Plates

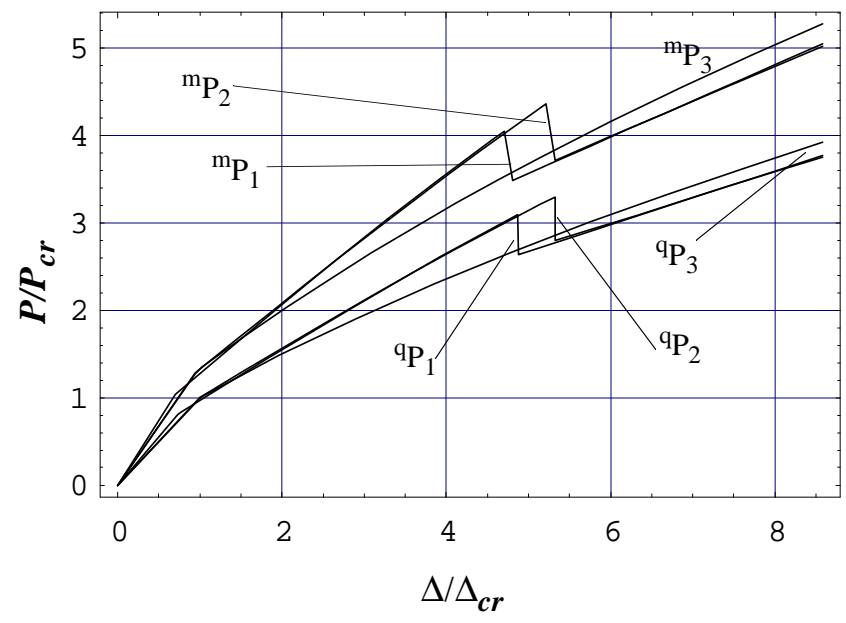

Fig. 3.34: Load Response of Unstiffened Quasi-isotropic and Metallic Plates

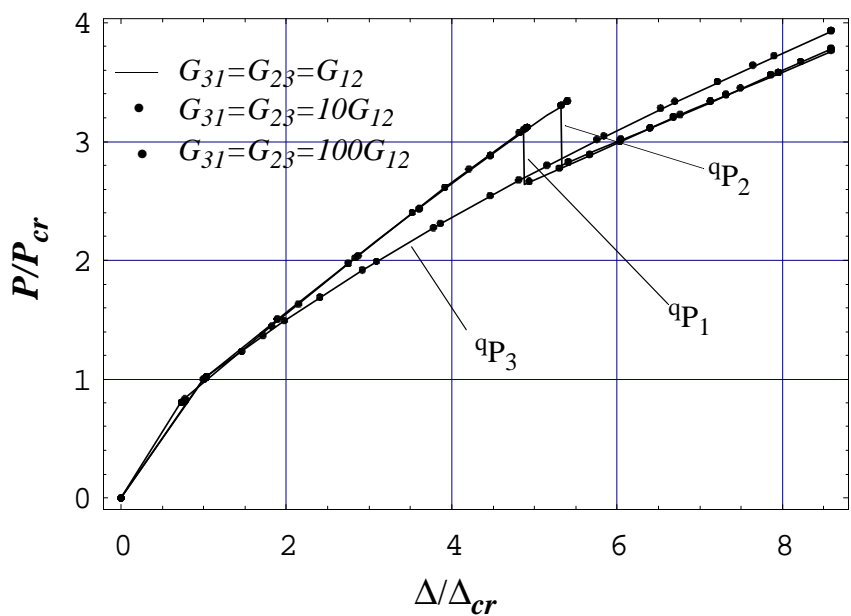

Fig. 3.36: Impact of Out-of-Plane Shear Stiffness on Load Response

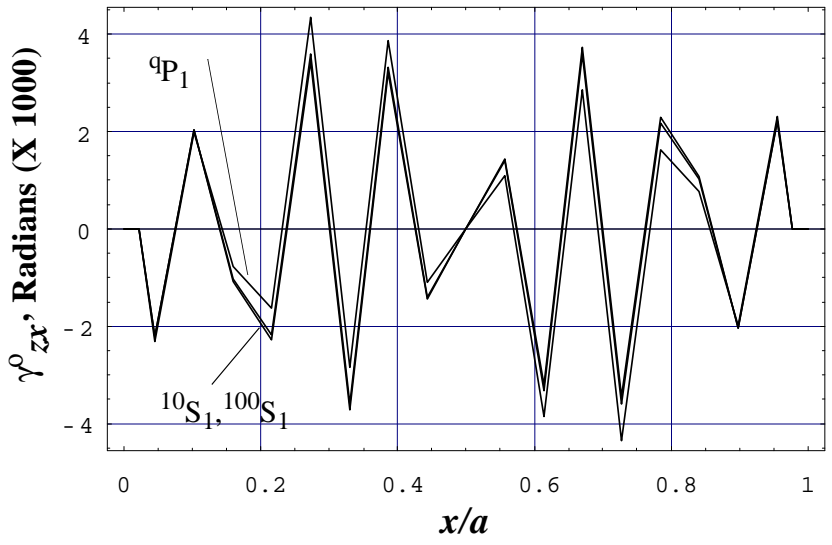

Fig. 3.38: Variation of Shear Strain $\gamma_{z x}^{0}$ along Vertical Mid-axis of Plates 


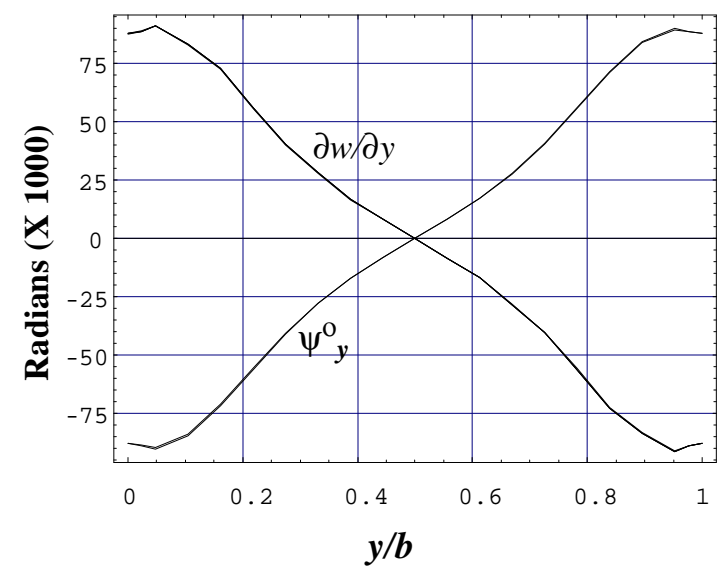

Fig. 3.37: Variation of $\psi^{o}{ }_{y}$ and $\partial w / \partial y$ along Horizontal Mid-axis of Plates

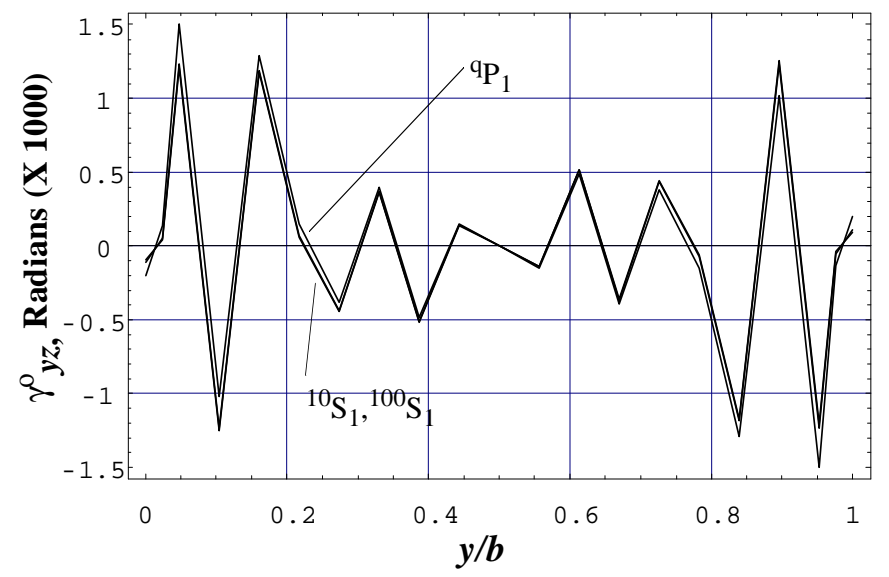

Fig. 3.40: Variation of Shear Strain $\gamma^{0} y z$ along Horizontal Mid-axis of Plates 


\section{DESCRIPTION OF EXPERIMENTS}

\subsection{INTRODUCTION}

With a view to validate the predictions of the finite element results, several experiments were conducted. These experiments were comprised of several stiffened as well as unstiffened plates subjected to uniform inplane end shortening. Considerable effort and thought were invested to ensure that the actual boundary conditions were close simulations of those applied in the corresponding finite element studies.

\subsection{TEST FIXTURE AND SETUP}

As discussed earlier, it is far more easy to simulate in the laboratory a clamped boundary condition along the loaded edge of a plate, as compared to simulating a simply supported boundary condition. Further, it is also true that simulation of a uniform end shortening along the loaded edge of a plate in the laboratory is more easy than trying to enforce a uniform force loading along the edge of the plate. Thus, it was decided to conduct uniform inplane end shortening tests on plates which were clamped along the loading edges and simply supported along other two edges. This was the motivation for the boundary conditions considered in the numerical studies of the previous chapter.

A schematic diagram of the fixture is shown Fig. 4.1. This figure is similar to Fig. 3.1 which has been described in detail in section 3.2. In addition to those details, Fig. 4.1 also shows the placement of thin strips of Teflon between the stiffeners and the knife edges. These layers served the purpose of reducing the friction between the simply supported edges and the plate. During the course of experiments it was discovered that the absence of this Teflon layer led to high friction between the knife edges and the plate, which manifested as a nonuniform prebuckling strain field. A detailed description of the fixture used for testing the plates can be found in [34]. 


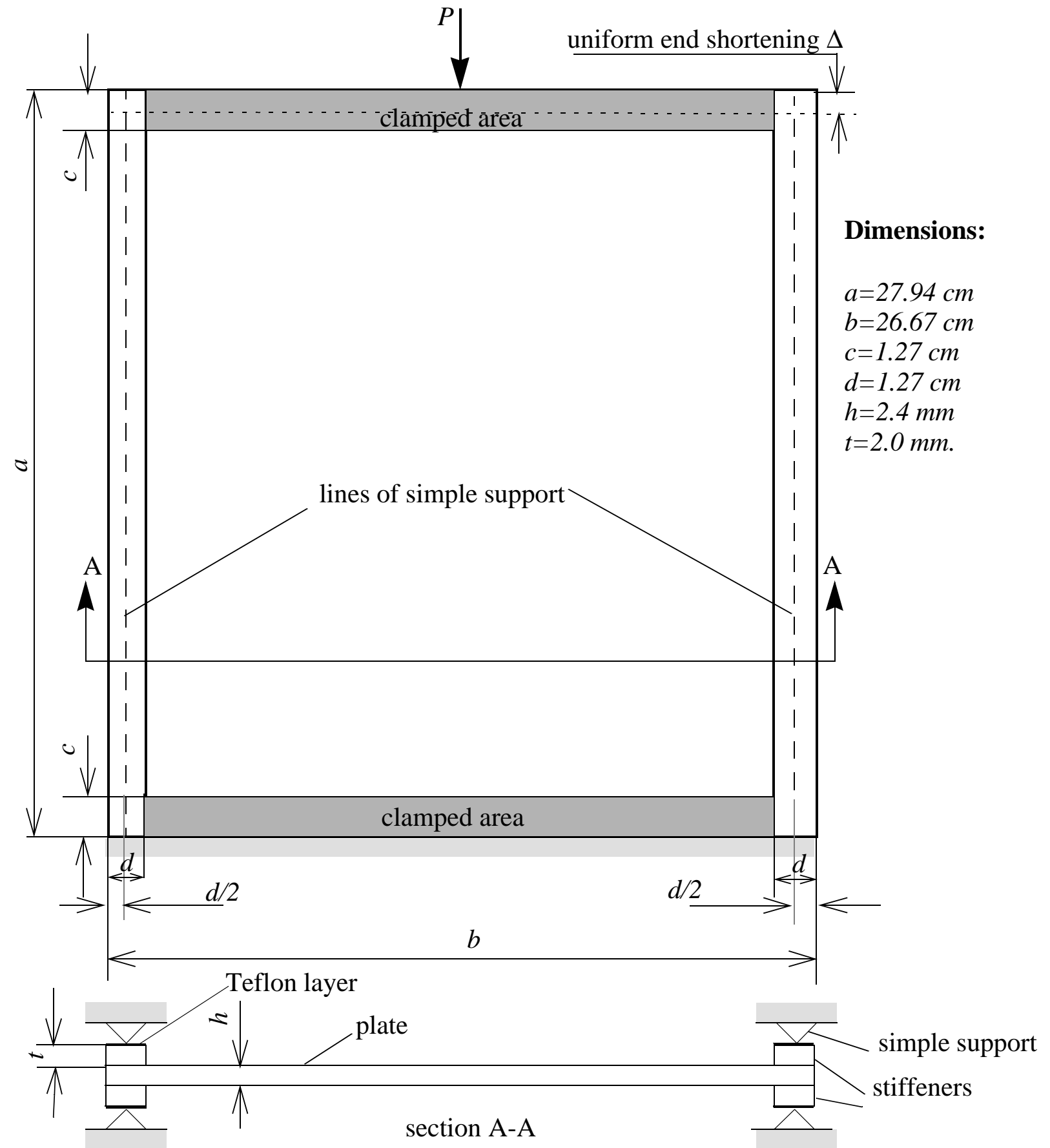

Fig. 4.1 - Schematic Diagram of the Fixture with Plate

Figure 4.2 illustrates the locations and numbering of the strain gages and DCDTs (direct current displacement transducers) used to measure plate displacement response. DCDT1 and DCDT2, the left and right DCDTs, respectively, were employed to sense the value of end shortening displace- 
ments. DCDT3, DCDT4, and DCDT5, the top, middle and bottom DCDTs, respectively, were used to sense the out-of-plane displacement of the plate at selected locations.

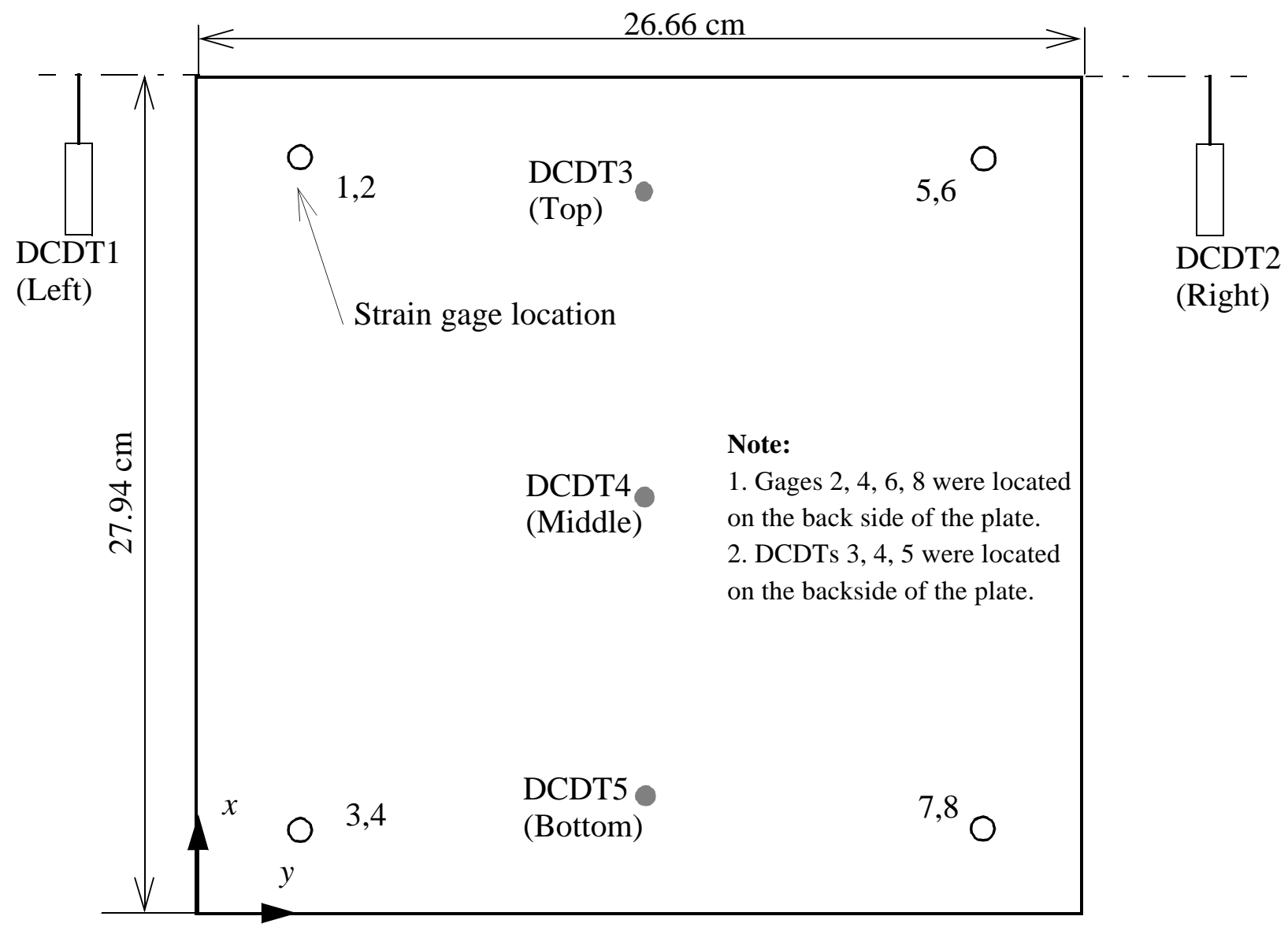

Fig. 4.2: Location of Strain Gages and DCDTs on Unstiffened Plates as seen from the Front Side

As shown in Fig. 4.2, eight strain gages were placed on the unstiffened plate. The locations of these gages have been numbered as 1 though 8. Gages 1, 3,5, and 7 were placed on the front side of the plate, while gages 2, 4, 6, 8 were placed on the back side of the plate. The coordinates of these gages, with respect to the axes system depicted in Fig. 4.2, are given in Table 4.1. Gages 1 and 2, 3 and 4, 5 and 6, and 7 and 8 were placed back-to-back, respectively, with the intention of monitoring the inplane and bending components of strains at these locations. It was reasoned that the occurrence of prebuckling bending of a symmetrically laminated plate could be detected by the presence of nonzero antisymmetric components of strains. The detection of any prebucklingbending strains was considered important, since that would have indicated that the plate was being inappropriately subjected to a bending moment at the clamped edges, in addition to a uniform end shortening displacement. 
While monitoring end shortening displacements, care was taken to ensure that at a given load the values of recordings made by DCDT1 and DCDT2 were close. This was done to ensure that the displacement boundary condition along the loading edge was uniform. The uniformity of the displacement was further ensured by monitoring and comparing the strain readings from gages 1,2 , 3,4 with those from 5, 6, 7, and 8. The location of the DCDTs with respect to the coordinate system shown in Fig. 4.2 are given in Table 4.1.

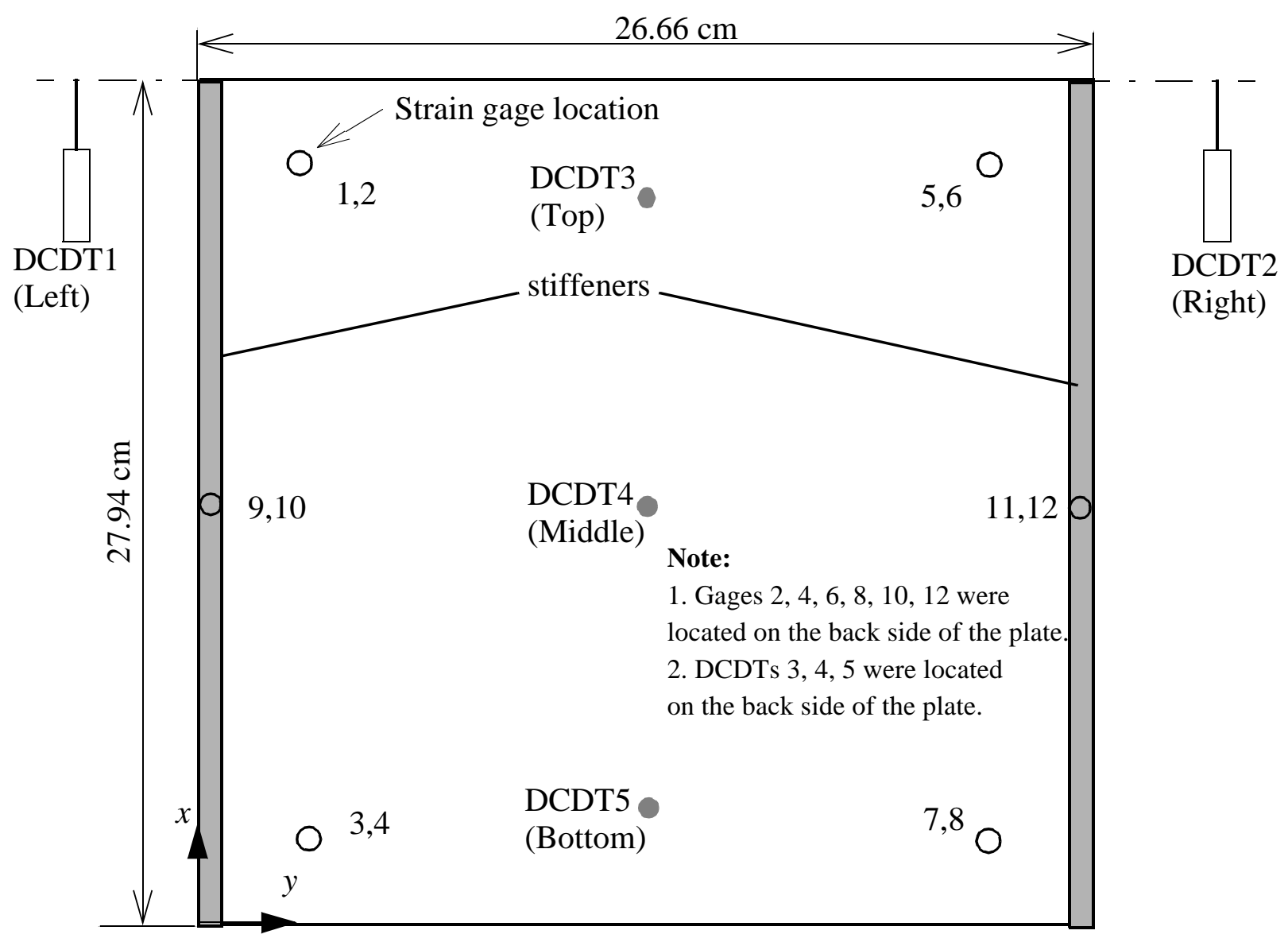

Fig. 4.3: Location of Strain Gages and DCDTs on Stiffened Plates as seen from the Front Side 


\begin{tabular}{||c|c|c|c|c|c|c|c||}
\hline \multicolumn{6}{|c|}{ Table 4.1: Position (mm) of Strain Gages and DCDTs on Unstiffened Plates. } \\
\hline \hline Coordinates & \multicolumn{3}{|c|}{ Strain Gages } & \multicolumn{3}{c|}{ DCDTs } \\
\hline \hline & 1,2 & 3,4 & 5,6 & 7,8 & 3 & 4 & 5 \\
\hline$x(\mathrm{~cm})$ & 24.77 & 3.175 & 24.77 & 3.175 & 20.57 & 14.63 & 7.620 \\
\hline$y(\mathrm{~cm})$ & 3.175 & 3.175 & 23.50 & 23.50 & 13.34 & 13.34 & 13.34 \\
\hline
\end{tabular}

Figure 4.3 shows the placement of strain gages and DCDTs on the stiffened panels. Table 4.2 lists the coordinates of the strain gages and DCDTs. As compared to the unstiffened panels, the stiffened panels had 12 strain gages instead of eight. The four extra gages, labelled as 9, 10, 11, 12 in Fig. 4.3, were placed on the steel stiffeners.

\begin{tabular}{|c|c|c|c|c|c|c|c|c|c|}
\hline \multirow[t]{2}{*}{ Coordinates } & \multicolumn{6}{|c|}{ Strain Gages } & \multicolumn{3}{|c|}{ DCDTs } \\
\hline & 1,2 & 3,4 & 5,6 & 7,8 & 9,10 & 11,12 & $\overline{3}$ & 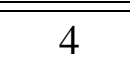 & 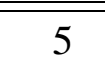 \\
\hline$x(\mathrm{~cm})$ & 23.40 & 4.445 & 23.40 & 4.445 & 13.97 & 13.97 & 20.57 & 14.05 & 7.577 \\
\hline$y(\mathrm{~cm})$ & 4.445 & 4.445 & 22.23 & 22.23 & 0.635 & 26.04 & 13.34 & 13.34 & 13.34 \\
\hline
\end{tabular}

In addition to DCDTs 3, 4, and 5 as shown in Figs. 4.2 and 4.3, the out-of-plane deflection patterns of the plates were also measured with the help of shadow Moire' [35] technique. This method was used to get a qualitative assessment of the overall out-of-plane deflection pattern of the plates. For this purpose one side of the plate was painted white after the placement of strain gages. A thin photographic film with a line density of 8 per mm, mounted on a $12.7 \mathrm{~mm}$ thick piece of plexiglass, was used as a reference grid. This reference grid was placed in front of the white surface of the plate and was exposed to an oblique white light source. The interference of the reference lines and their shadows produced a set of fringe patterns which was recorded by a television camera. Figure 4.4 is a schematic representation of the instrumentation associated with the measurement of out-of-plane deflection of the plate. 


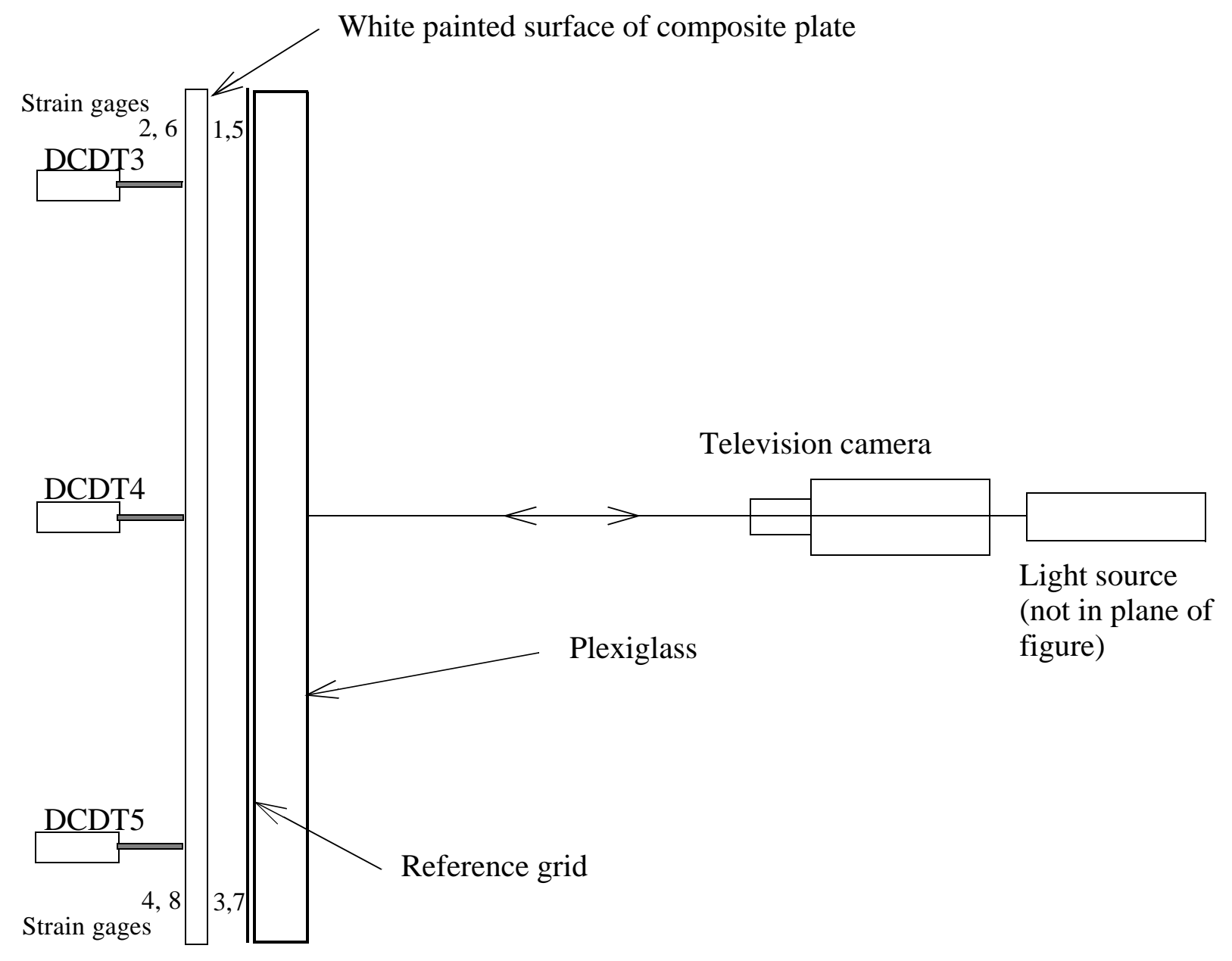

Fig. 4.4: Schematic Representation of Instrumentation Associated with the Measurement of Out-of-Plane Deflections of an Unstiffened Plate

Figure 4.5 is a schematic representation of the overall layout of the test equipment. The plate was loaded with a screw-driven Tinius Olsen loading frame. As seen in the figure, the signals from the strain gages were sent to a strain gage conditioner for amplification purposes. To minimize electro-magnetic noise, shielded strain gage cables were used. The output from the strain gage conditioner was then connected to an AMUX-64T multiplexer from National Instruments. The multiplexer was also connected to signals from the load cell and the DCDTs. The output from the multiplexer was then sent to a National Instruments Labview A/D converter which digitized the multiplexed analog data, translated it into numbers, and then stored it on the hard drive of a MacIntosh computer. During the experiments a television camera was used to record shadow Moire' fringe patterns. Additionally, a Nikon still photographic camera was used to take intermit- 
tent snap shots of the fringe patterns.

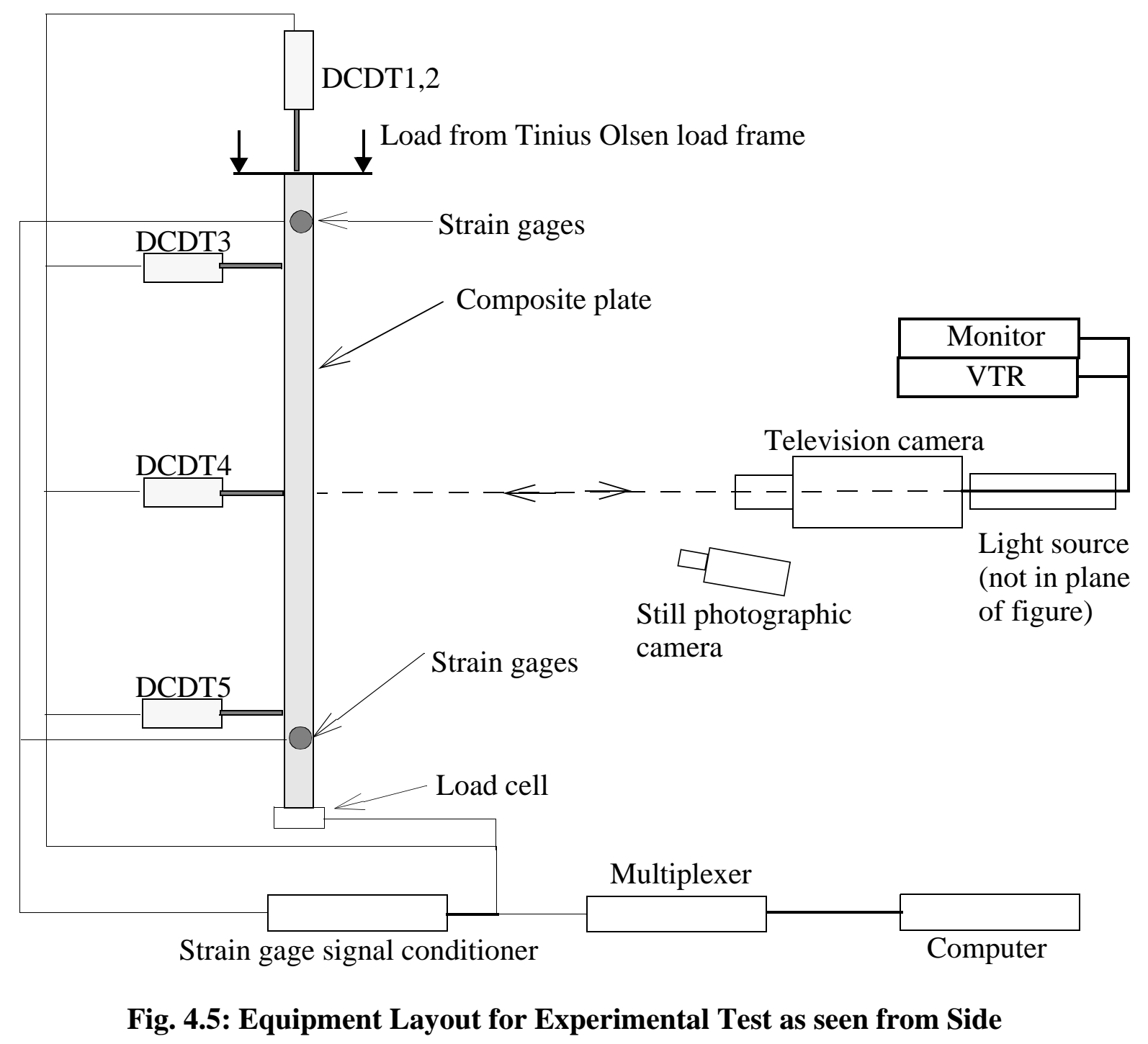

\subsection{FABRICATION OF COMPOSITE PLATES}

Composite plates were fabricated using the hand layup technique. Following the hand layup, the laminates were subjected to heat and pressure in a vacuum environment. The prepreg used for this purpose was NARMCO-BASF 5245C-G40-600. The material system comprised of C600 fibers impregnated with 5245 bismaleimide (BMI) resin. The details of the temperature and pressure cycles employed for curing and postcuring purposes are shown in Fig. 4.6. 

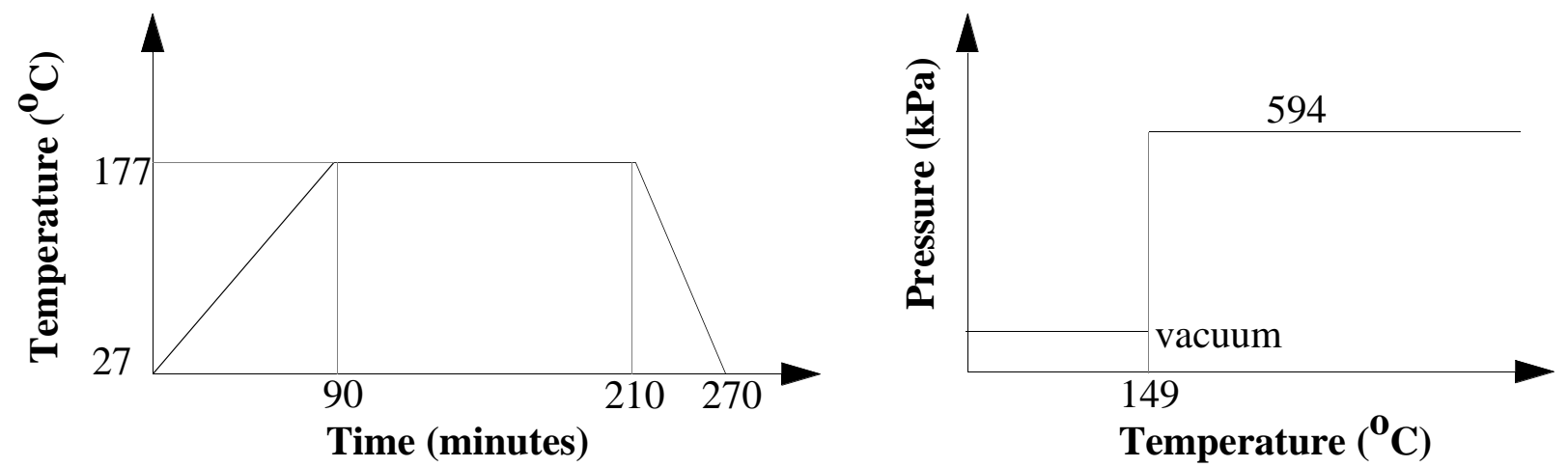

Fig. 4.6: Prescription of Heat and Pressure during the Curing of BMI Laminate

\subsection{CLOSURE}

The experimental setup has been described. The following chapter compares the experimental results with the finite element calculations. 


\section{COMPARISON OF EXPERIMENTAL AND NUMERICAL DATA}

\subsection{MATERIAL CHARACTERIZATION}

Four sets of experiments were conducted to characterize the graphite-epoxy material system used to make the plates. In all of these experiments flat rectangular strips of laminates were tested in tension. The typical shape of a test coupon is shown in Fig. 5.1.

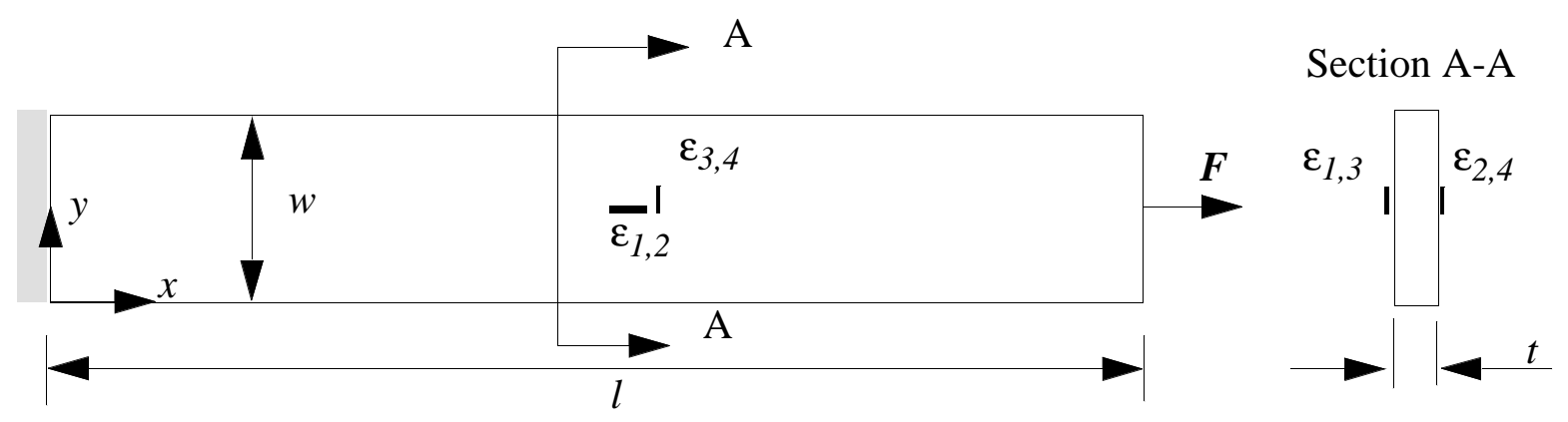

Fig.: 5.1 Details of the Test Coupon

In Fig. 5.1, $l, w$, and $t$ designate the length, width, and the thickness of the test coupon, respectively. A tensile load $F$ was applied at one end of the specimen, while the other end was held rigidly fixed. Four strain gages, two on each side of the coupon, were placed to record the strains in the loading and transverse directions. Gages were placed on both sides of the coupon with a view to monitor the bending and torsion of the coupon, if any. The details of these four experiments are given below.

\subsection{1 $[90]_{4}$ Test Coupon}

A $[90]_{4}$ coupon was used to evaluate the modulus of the composite material in the direction transverse to fiber direction. The coupon was loaded in transverse direction. The relation used to evaluate transverse modulus, $E_{2}$, was

$$
E_{2}=\frac{\text { Stress }}{\text { Strain }}=\frac{F /(w t)}{\varepsilon}
$$


Figure 5.2 shows the stress strain response relation for the $[90]_{4}$ test coupon. From this relation the value of $E_{2}$ was found to be $8.75 \mathrm{GPa}$.

\subsection{2 $[0]_{4}$ Test Coupon}

A $[0]_{4}$ coupon was used to used to evaluate the modulus of composite material in the fiber direction, and also the major Poisson's ratio, $v_{12}$. However, during the actual test, load data could not be recorded accurately because of high noise levels on the load channel. Thus, the data from this test were used to compute only the Poisson's ratio, $v_{12}$. Figure 5.3 shows the variation of transverse strain as the strain in fiber direction in the $[0]_{4}$ laminate is increased. The value $v_{12}$ was found to be 0.27 .

\subsection{3 $\left[90_{2} / 0 / 90 / 0_{1 / 2}\right]_{\mathrm{s}}$ Test Coupon}

A cross-ply $\left[90_{2} / 0 / 90 / 0_{1 / 2}\right]_{\mathrm{S}}$ was used to compute the modulus, $E_{l}$ in the fiber direction. This particular laminate was chosen for purposes of determination of $E_{l}$ because it was already available in the testing laboratory. The average modulus, $E_{c}$, of such a laminate in the $0^{\circ}$ direction, based on the rule of mixtures, can be written as

$$
E_{c}=\frac{6 E_{2}-3 E_{1}}{9}
$$

From this relation, $E_{1}$ can be computed if $E_{2}$ and $E_{c}$ are known. The value of $E_{c}$, as shown in Fig. 5.4, was found to be as $58.60 \mathrm{GPa}$. Thus, the modulus of the material in fiber direction was determined as $165 \mathrm{GPa}$.

\section{$5.1 .4[ \pm 45]_{\mathrm{S}}$ Test Coupon}

An angle ply coupon was tested in tension for the determination of shear modulus, $G_{12}$, of the unidirectional material. From classical lamination theory, the stress-strain relations for a $[ \pm 45]_{\mathrm{S}}$ laminate, when subjected to uniform unidirectional inplane loads, can be written as 


$$
\left[\begin{array}{l}
N_{x} \\
N_{y}
\end{array}\right]=\left[\begin{array}{l}
- \\
\sigma_{x} \\
-\sigma_{y}
\end{array}\right] t=\left[\begin{array}{ll}
A_{11} & A_{12} \\
A_{12} & A_{22}
\end{array}\right]\left[\begin{array}{c}
o \\
\varepsilon_{x}^{o} \\
\varepsilon_{y}^{o}
\end{array}\right]
$$

Here, $N_{x}$ and $N_{y}$, as defined in Chapter 2, are stress resultants in $x$ and $y$ directions, respectively and $\bar{\sigma}_{\mathrm{x}}$ and $\bar{\sigma}_{\mathrm{y}}$ are the average normal stresses in the laminate in $x$ and $y$ directions, respectively. Also, $\varepsilon^{o}{ }_{x}$ and $\varepsilon^{o}{ }_{y}$ are midplane normal strains in $x$ and $y$ directions, respectively. Since the tensile test is unidirectional in nature, the value of $\bar{\sigma}_{y}$ is zero. For a $\left[{ }_{ \pm} 45\right]_{\mathrm{s}}$ laminate, the elements of extensional stiffness matrix $A$ can be written as

$$
\begin{gathered}
A_{11}=A_{22}=(t / 4)\left[Q_{11}+2\left(Q_{12}+2 Q_{66}\right)+Q_{22}\right] \\
A_{12}=(t / 4)\left[Q_{11}+2\left(Q_{12}-2 Q_{66}\right)+Q_{22}\right]
\end{gathered}
$$

where the definitions of reduced stiffnesses of the material $Q_{11}, Q_{22}, Q_{12}$, and $Q_{66}$ are given in Ref. [36]. Substituting these relations in the constitutive equations for the laminate as given above yields

$$
\bar{\sigma}_{x}=2 Q_{66}\left(\varepsilon^{o}{ }_{x}-\varepsilon^{o} y\right)
$$

Since $Q_{66}$ equals $G_{12}$, this relation can be rewritten as

$$
G_{12}=\left(\bar{\sigma}_{x} / 2\right) /\left(\varepsilon_{x}^{o}-\varepsilon_{y}^{o}\right)
$$

Figure 5.5 is a plot $\left(\bar{\sigma}_{x} / 2\right)$ as a function of the difference in strains $\left(\varepsilon^{o} x^{-\varepsilon^{o}}\right)$. The slope of this response line is the shear modulus for the unidirectional material. In the actual test the responses of strain gages affixed to the front and the back sides of the test coupons were slightly different. This difference can be seen by the presence of two different sets of data in Fig. 5.5. The average value of shear modulus obtained from these two sets of data was found to be $4.75 \mathrm{GPa}$. 


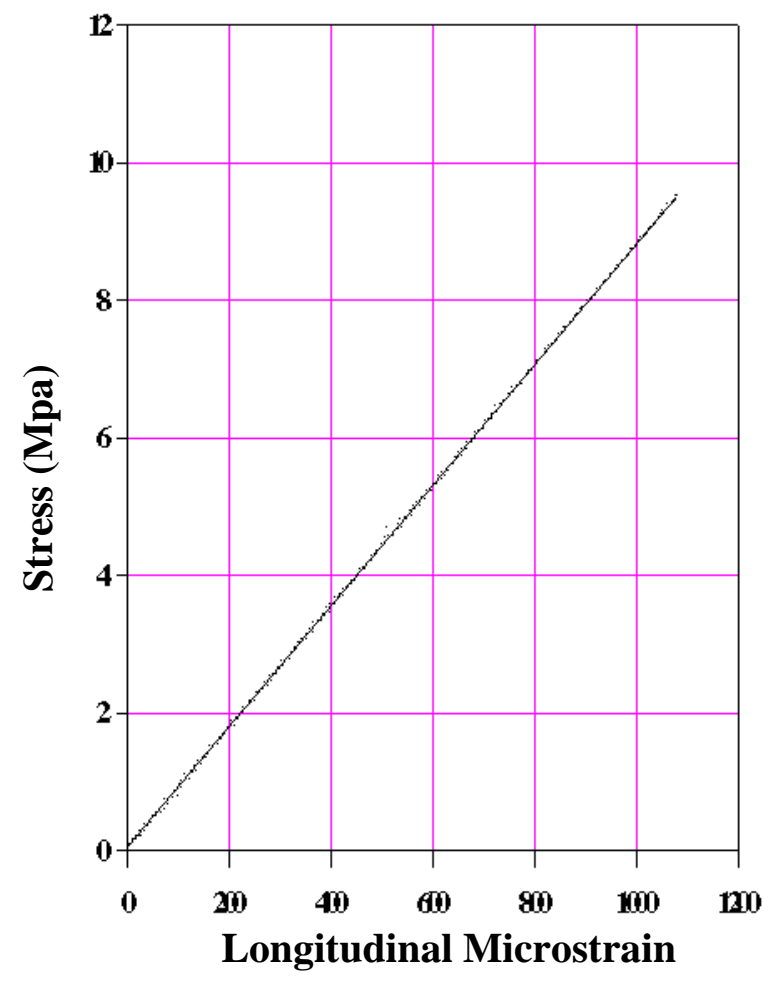

Fig 5.2: Stress-Strain Response for the [904] Laminate

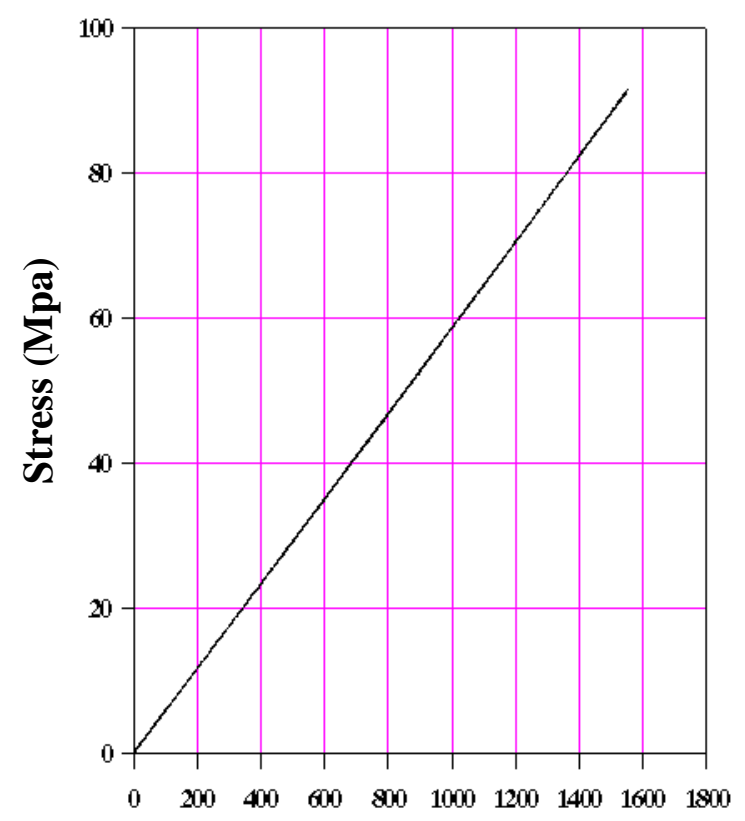

Microstrain

Fig. 5.4: Stress-Strain Response for the $\left[90_{2} / 0 / 90 / 0_{1 / 2}\right]_{\mathrm{S}}$ Laminate

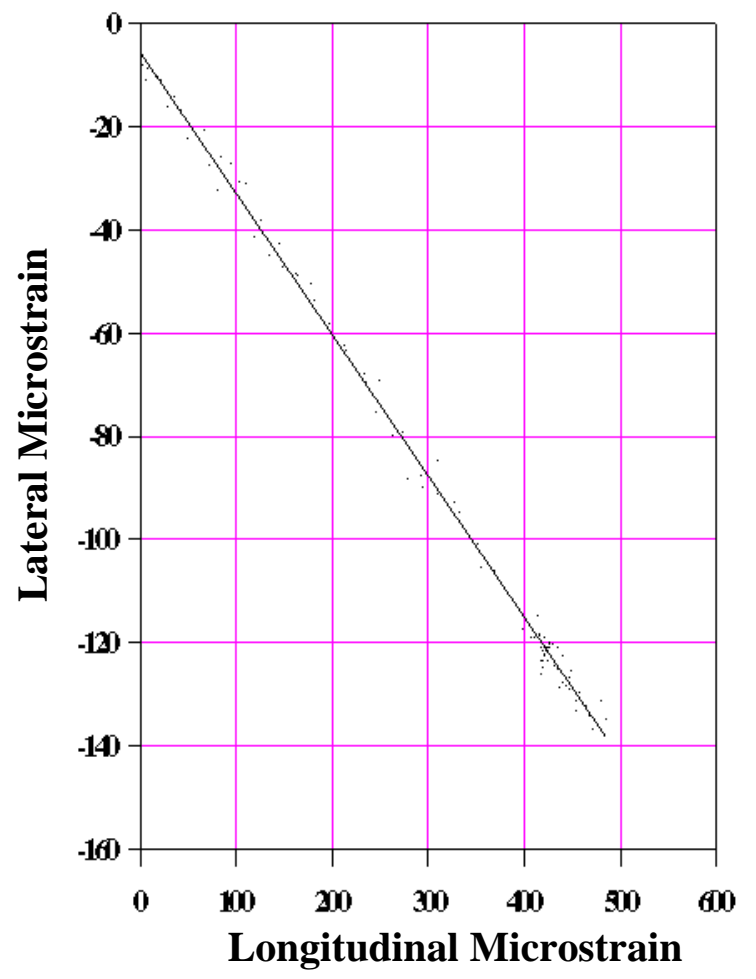

Fig. 5.3: Poisson-Strain Response for the $\left[0_{4}\right]$ Laminate

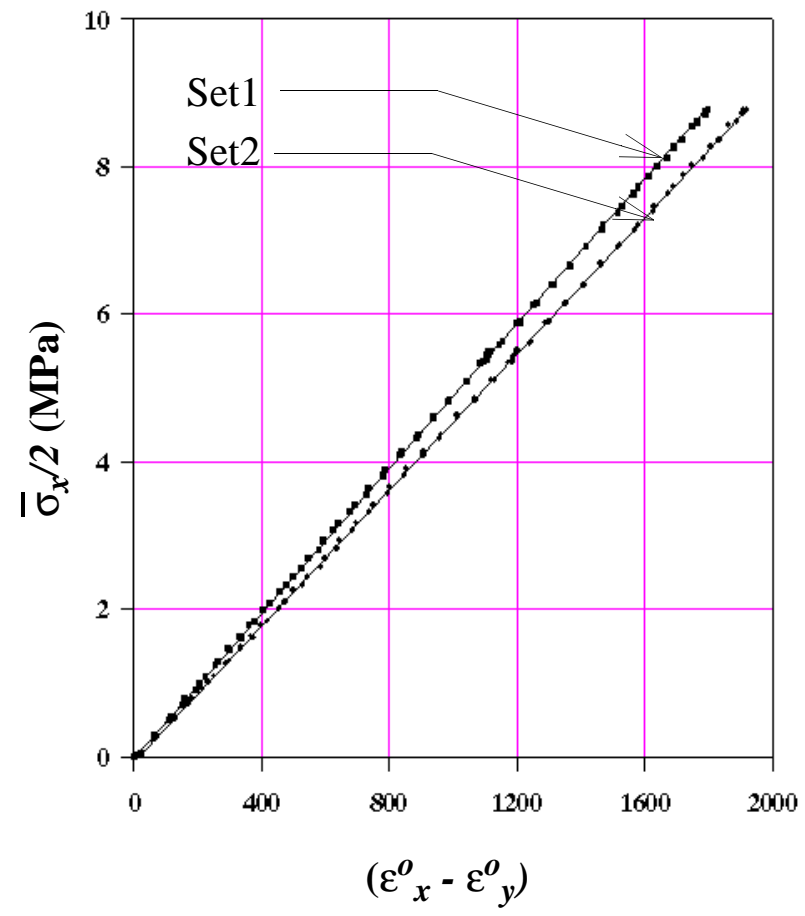

Fig. 5.5: $\bar{\sigma}_{x} / 2 \operatorname{versus}\left(\varepsilon^{o}{ }_{x}-\varepsilon^{o}\right)$ for the $[ \pm 45 / 45]_{S}$ Laminate 


\subsection{EXPERIMENTAL RESULTS FOR PLATES SUBJECTED TO COMPRESSION}

Overall, six plates were tested. Three of these plates were unstiffened, while the rest had steel stiffeners. Details regarding the use of stiffeners on these six plates are given in the following table:

\begin{tabular}{|c|c|c|c|}
\hline \multicolumn{4}{|c|}{ Table 5.1: Details of Plate Used For Testing } \\
\hline Plate No. & Plate Layup & Stiffeners & Other Details \\
\hline 1 & {$\left[ \pm 45 / 0_{2}\right]_{\mathrm{s}}$} & No & Material Properties: \\
\hline 2 & {$\left[ \pm 45 / 0_{2}\right]_{\mathrm{s}}$} & Yes & $E_{1}=165 G P a$ \\
\hline 3 & {$[ \pm 45 / 0 / 90]_{\mathrm{S}}$} & No & $E_{2}=8.75 \mathrm{GPa}$ \\
\hline 4 & {$[ \pm 45 / 0 / 90]_{\mathrm{S}}$} & Yes & $v_{12}=0.27$ \\
\hline 5 & {$\left[ \pm 45 / 90_{2}\right]_{\mathrm{s}}$} & No & Plv thickness $=0.30 \mathrm{~mm}$ \\
\hline 6 & {$\left[ \pm 45 / 90_{2}\right]_{\mathrm{s}}$} & Yes & Stiffener thickness $=2.0 \mathrm{~mm}$ \\
\hline
\end{tabular}

All the remaining details for these plates concerning their geometry, boundary conditions, and placement of external loads can be viewed in Fig. 4.1. For computational purposes, it was assumed that the plates had no out-of-plane imperfection. The output of the finite element analysis discussed in Chapter 2 was then processed and compared to the experimental data acquired. In this section, the experimental data are presented, analyzed, and compared to finite element analysis predictions. While conducting the experiments, care was taken while loading the plates into deep postbuckling range. This was done to test the validity of the finite element analysis prediction that the event of secondary buckling would or would not occur when these clamped-simply supported plates are in unidirectional compression. The overall predictions stood verified through experimental observations. The specific details of the tests conducted are given below. 


\subsubsection{The $[ \pm 45 / 0 / 90]_{\mathrm{S}}$ Plates}

Two plates with a quasi-isotropic layup were tested. One of these was unstiffened, while the other one had stiffeners. The details of these plates can be found in Table 5.1 and section 4.2.

\subsubsection{The $[ \pm 45 / 0 / 90]_{\mathrm{S}}$ Unstiffened Plate}

Figure 5.6, 5.7, 5.8, and 5.9 show the experimental data acquired while loading the $[ \pm 45 / 0 / 90]_{\mathrm{S}}$ unstiffened plate. These figures also show the respective finite element predictions. Figures 5.6 and 5.7 show the strain response of the plate, while Figs. 5.8 and 5.9 show the displacement response. Figure 5.6 shows the midplane component of the strains in the plate. The midplane component of the strain corresponding to gages 1 and 2 is defined as:

$$
\varepsilon_{1 / 2 \_ \text {midplane }}=\left(\varepsilon_{1}+\varepsilon_{2}\right) / 2,
$$

where the locations of gages 1 and 2 are shown in Fig. 4.2. Comparison of these midplane quantities with the finite element prediction reveals how accurately the finite element code captures the influence of extensional stiffness matrix $A$ on the deflection response of the plate.

Figure 5.7 shows the bending components of strains in the plate. These have been typically computed by taking half of the difference between the gage readings on opposite sides of the plate. Thus, $\varepsilon_{1 / 2 \_ \text {bending }}$ was computed as:

$$
\varepsilon_{1 / 2 \_ \text {bending }}=\left(\varepsilon_{1}-\varepsilon_{2}\right) / 2,
$$

This computed measure can also be interpreted as the strain in the plate due to its curvature. Thus,

$$
\varepsilon_{1 / 2 \_ \text {bending }}=\kappa_{x}^{o} h / 2,
$$

where $h$ is the overall plate thickness, and $\kappa^{o}{ }_{x}$ is the curvature of the plate in $x$ direction. Comparison of this measure with its finite element calculation reveals how accurately the finite element code captures the influence of bending matrix $D$ on the deflection response of the plate. 


\section{Prebuckling}

The prebuckling inplane stiffness of the $[ \pm 45 / 0 / 90]_{\mathrm{S}}$ unstiffened laminate, based on the classical lamination theory, was computed to be $62.2 \mathrm{GPa}$. The experimental values for this stiffness as measured in Figs. 5.6 and 5.8 were found to be 58.1 GPa and $61.1 \mathrm{GPa}$, respectively. These experimental observations are in good agreement with the finite element predictions of 62.2 GPa. It is also seen through Fig. 5.8 that the loading of the plate was fairly uniform, as the responses of the two DCDTs were quite close to each other. The small amount of deviation in the initial response of the DCDTs may be attributed to some slack in the fixture, which was eliminated as the inplane compressive load was increased.

The plates were fairly flat to begin with. This can be concluded by looking at Figs. 5.7 and 5.9. Figure 5.9 shows that the out-of-plane deflection of the plates at the three DCDT locations was almost zero in the prebuckling range. This is also seen in Fig. 5.7, which is a plot of bending strains as a function of increasing loads. These were close to zero during the prebuckling load conditions.

\section{Buckling}

The buckling load for this plate as predicted by the finite element analysis was $26 \mathrm{kN}$. Analysis of experimental data showed this value to be $23 \mathrm{kN}$. This value was found by finding the point of intersection of prebuckling and postbuckling paths in Fig. 5.7. The Moire' fringe patterns showed that the buckling pattern had one half-wave in both $x$ and $y$ directions. This is consistent with finite element predictions.

\section{Postbuckling}

As the inplane compression load was increased, it is seen in Fig. 5.9 that the out-of-plane deflections of the plate after buckling grew monotonically. However, as expected, no secondary buckling event occurred. This confirmed the finite element prediction that an unstiffened 
$[ \pm 45 / 0 / 90]_{\mathrm{S}}$ plate would not experience secondary buckling. Figure 5.9 also shows that for a given inplane compressive load, the out-of-plane deflections as predicted by finite element analysis were in reasonable agreement with experimental measurements.

Observation of end shortening response of the plate in the postbuckling range reveals that the overall stiffness of the plate dropped by a significant factor after the plate buckled into its primary buckling configuration. This is reflected in a reduced slope of the end shortening relation seen in Fig. 5.8. A similar trend can also be seen in the midplane strain response of the plate shown in Fig. 5.6. The deflection data indicated the postbuckling stiffness was $35.2 \mathrm{GPa}$, while the strain data indicated $31.6 \mathrm{GPa}$. The finite element predictions gave $30.5 \mathrm{GPa}$.

In the postbuckling range the contribution of plate's curvature to the overall strain field should be nonzero. This is seen in Fig. 5.7. Once again the correlation between experiment and numerical analysis is reasonably close. Since the values of $D_{16}$ and $D_{26}$ terms in the bending matrix $D$ for this laminate were significantly large, it was seen that the out-of-plane deflection patterns of the plate were not symmetric with respect to the horizontal and vertical planes of geometric symmetry. This experimental observation, as seen in the strain gage readings and also the Moire' fringe patterns, was once again consistent with the finite element analysis predictions.

The sign of the bending strains should be noted. The sign conventions were chosen such that a deflection towards the Moire' reference grid (see Fig. 4.4) was considered positive. That is, a positive out-of-plane deflection of the plate was recorded by DCDTs when their plungers moved out of the barrels. Bending strains, which have been defined earlier in Eq. 34, would assume positive values if the strains on the surface of the plate closer to reference grid, e.g., gage 1, were more positive than the strains on the opposite side of the plate, e.g., gage 2. The figure shows positive bending strains and negative out-of-plane displacements. This was due to the severe condition that occurred at the strain gage locations near the clamped boundary where the plate deflected away from the reference grid. 


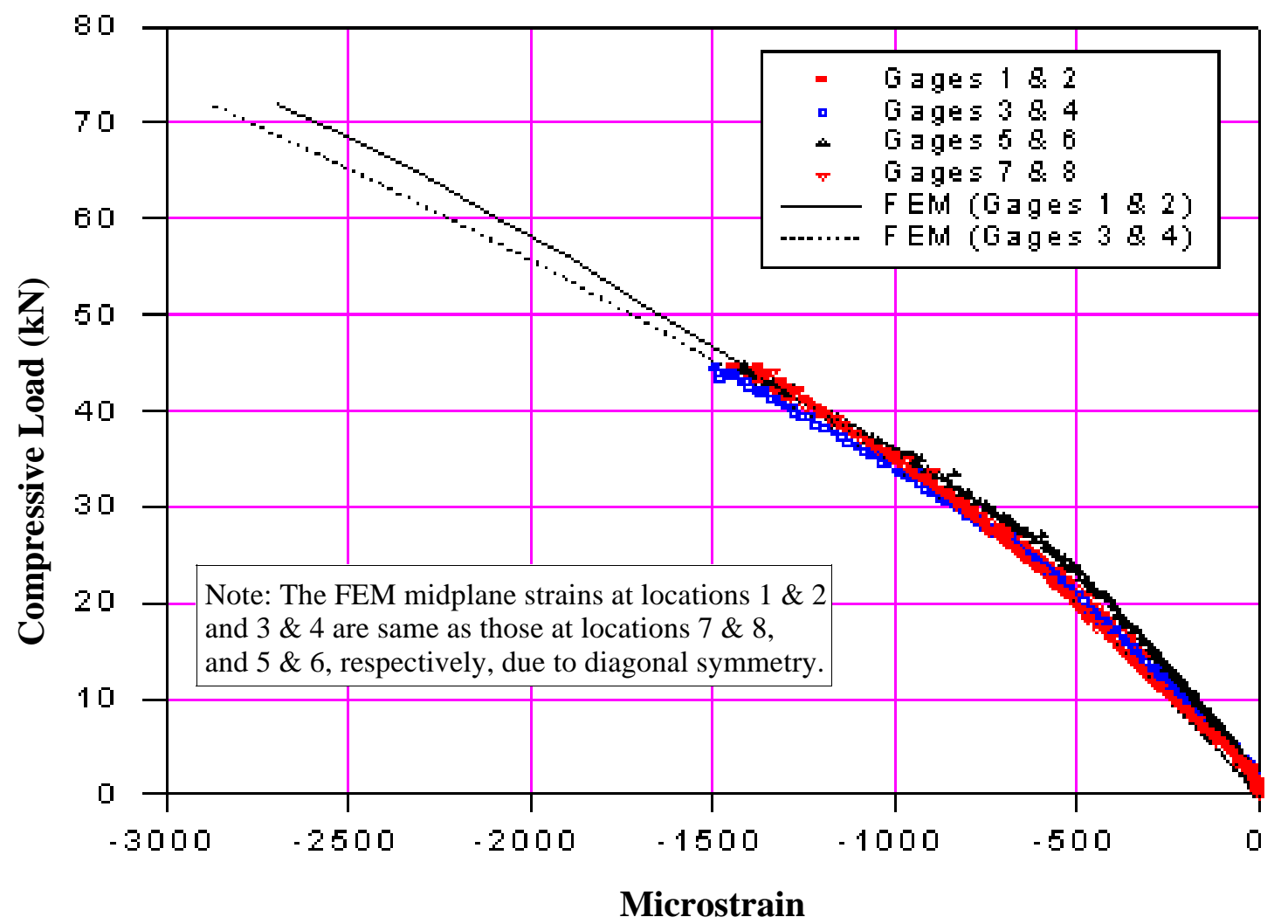

Fig. 5.6: Midplane Strains in Unstiffened $[ \pm 45 / 0 / 90]_{\mathrm{S}}$ Plate

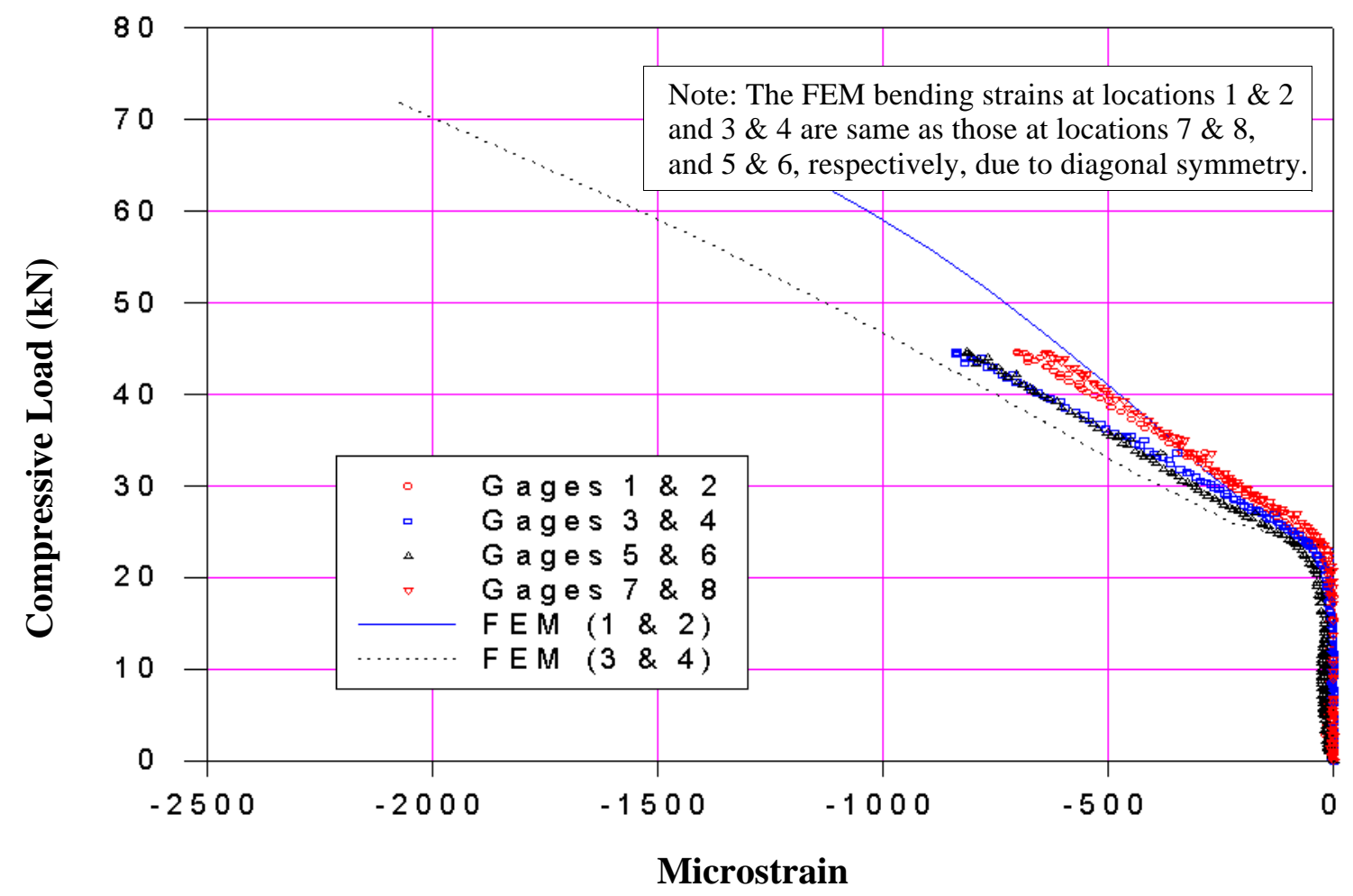

Fig. 5.7: Bending Strains in Unstiffened $[ \pm 45 / 0 / 90]_{\mathrm{S}}$ Plate 


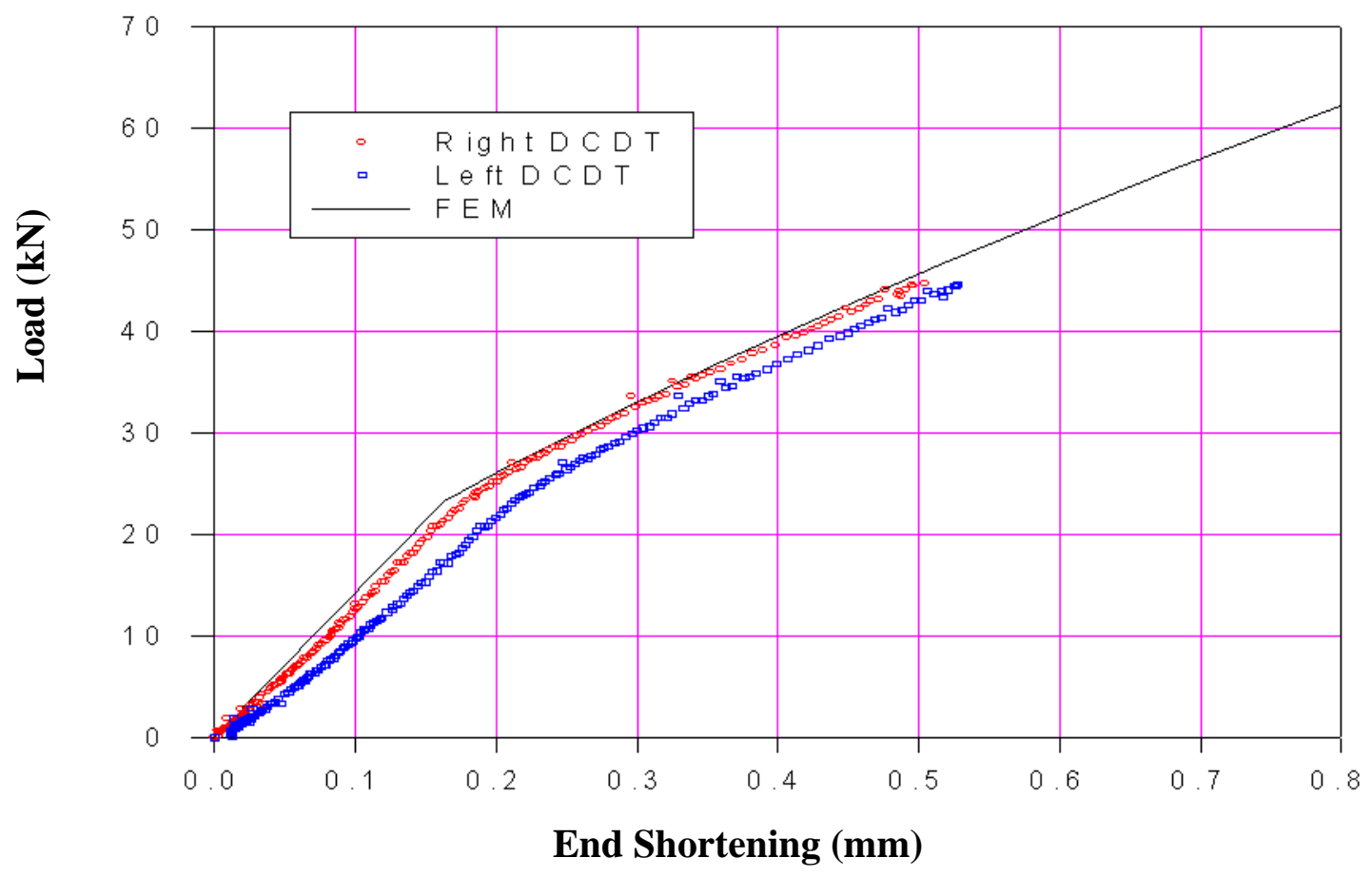

Fig. 5.8: End Shortening Response for Unstiffened $[ \pm 45 / 0 / 90]_{S}$ Plate

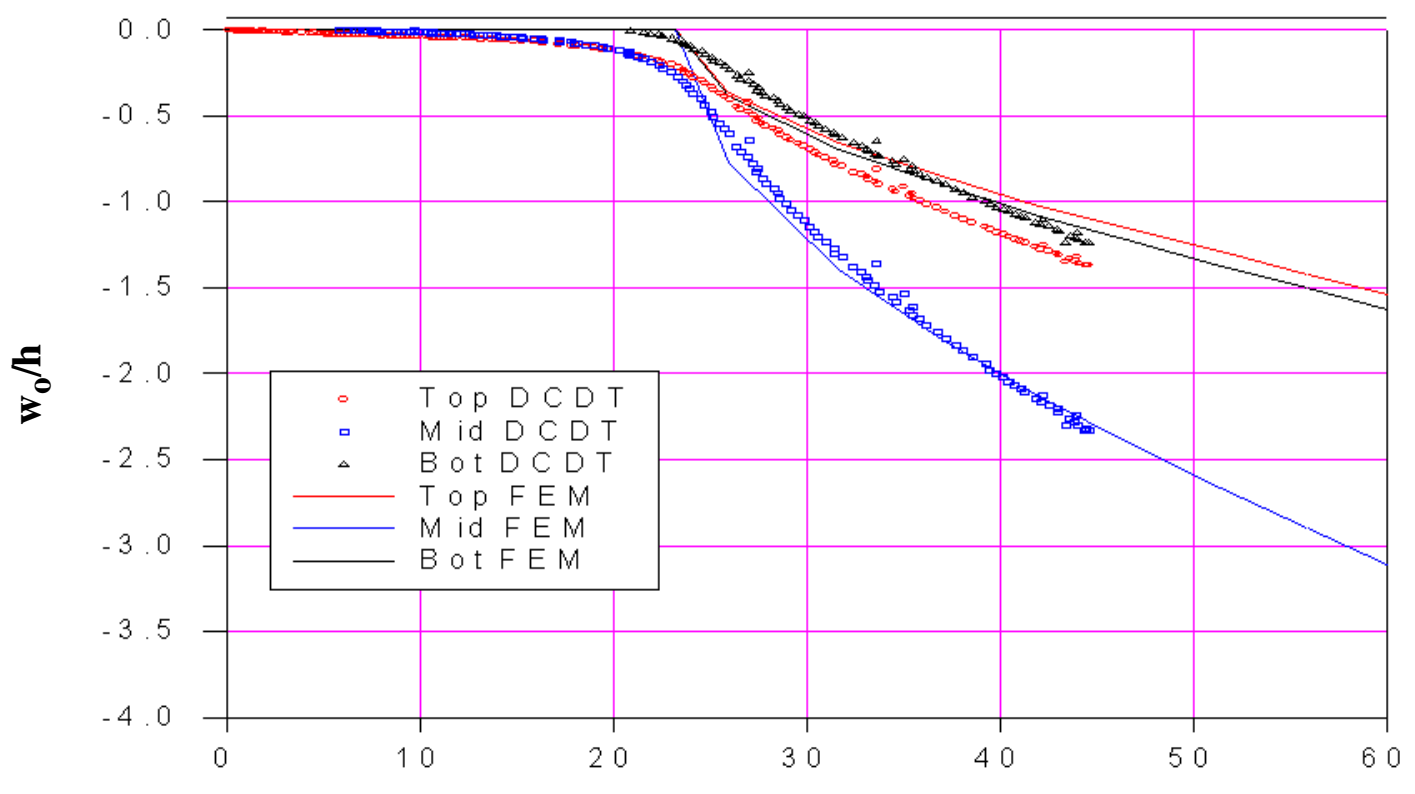

Compressive Load $(\mathrm{kN})$

Fig. 5.9: Deflection Response For Unstiffened $[ \pm 45 / 0 / 90]_{S}$ Plate 


\subsubsection{The $[ \pm 45 / 0 / 90]_{\mathrm{S}}$ Stiffened Plate}

Figures 5.10 through 5.14 show various responses of the stiffened $[ \pm 45 / 0 / 90]_{\mathrm{S}}$ plate subjected to inplane compression. Figures 5.10, 5.11, and 5.12 depict the midplane and bending strain responses of the plate. Figure 5.13 shows the end shortening response of the plate. Finally, Fig. 5.14 shows the out-of-plane deflection response of the stiffened $[ \pm 45 / 0 / 90]_{\mathrm{S}}$ plate. In all these figures both experimental as well as finite element results are presented.

\section{Prebuckling}

Figure 5.13 shows that the prebuckling loading of the plate was fairly uniform. This can be stated since the response of the right and left DCDTs appear almost parallel, for almost the entire load range. A small deviation from this trend, however, is visible for very low load levels, which was possibly due to the presence of initial slack in the fixture. The plate was also loaded uniformly in the thickness direction. This can be said since the responses of the strain gages on front as well as on the back of the plate were almost identical in the prebuckling range, as can be seen in Figs. 5.10-5.12. Figure 5.14, which shows the out-of-plane deflection response of the plate, indicates that the plate was initially almost flat, since the prebuckling out-of-plane deflections were close to zero. The prebuckling stiffness of the stiffened plate from Figs. 5.10 and 5.13 was found to be $80.9 \mathrm{GPa}$ and $75.5 \mathrm{GPa}$, respectively. This compared well with the numerically evaluated value of $77.5 \mathrm{GPa}$.

\section{Buckling}

The buckling point of the this particular plate was found to be $47 \mathrm{kN}$. The predicted value was $44 \mathrm{kN}$. Also, as expected, the Moire' fringe patterns of the buckled plate showed that the plate's buckling pattern had one half-wave in each $x$ and $y$ directions. Figure 5.15 shows the pattern of out-of-plane deflections just after the plate buckled. The load measured at this condition, as seen in Fig. 5.15, was found to be equivalent to 0.84 volts, which corresponded to $44.9 \mathrm{kN}$, since each volt at this load level corresponded to $53.5 \mathrm{kN}$. 


\section{Postbuckling}

As the plate was loaded into the postbuckling range, the amplitude of the deflection patterns increased monotonically with increasing loads up to a certain extent. This can be seen in Fig. 5.14. However, beyond a certain load level, the plate almost instantly changed its configuration, and developed a shape that had two half-waves in the loading direction and one half-wave in the $y$ direction. The video recording of the experiment revealed that the actual snap-through event occurred when the inplane load applied to the plate was $102 \mathrm{kN}$. This value was remarkably close to its the predicted value of $105 \mathrm{kN}$. Figure 5.16 shows the Moire' fringe pattern of the outof-plane deflections of the plate just after the snap-through event. At this load level each volt of reading corresponded to $267.5 \mathrm{kN}$. Also, the load drop associated with this event was experimentally found to be $5.35 \mathrm{kN}$. This value was evaluated by noticing the drop as recorded by the video camera. This value was once again close to the finite element prediction of $6.4 \mathrm{kN}$.

The event of secondary buckling can also be seen in Fig. 5.14. It can be seen in the figure that following buckling, the out-of-plane deflections at all three locations of DCDTs gradually rose with increasing load. However, when the external load exceeded secondary buckling load, the deflection at the middle of the plate dropped to a value close to zero. Also, the deflection at the top DCDT location remained positive and that at the bottom DCDT location became negative. The rapid transition in the signs and values of the out-of-plane displacements at these three locations indicated that after buckling into the secondary configuration the plate had two halfwaves in the loading direction and one half-wave in the transverse direction. It is also seen in Fig. 5.14 that the correlation between numerical analysis and experimental data was reasonably close.

On the end shortening response curve, Fig. 5.13, the event of secondary buckling is not clearly marked. Ideally, the end shortening curve should exhibit a sudden drop in the load at the point of secondary buckling. However, the experimental data does not show this drop clearly. This can be attributed to the presence of high noise-signal ratios that were found to exist at for values external loads exceeding $53.5 \mathrm{kN}$. This, in turn, was because in this range a different load switch was chosen to record load signals, where the gain in signal was less by a factor of 5 . 
The midplane strain response of the plate in postbuckling range is shown in Fig. 5.10. Very much similar to the unstiffened plate, the slope of this relation decreases after the plate buckled in its primary configuration. The strain data of Fig. 5.10 indicate the postbuckling stiffness was 58.2 GPa, while the deflection data yielded 59.5 GPa. The finite element calculations gave 50.0 $\mathrm{GPa}$, a somewhat lower value. The bending strain response of the plate has been shown in Figs. 5.11 and 5.12. Here too, as in an unstiffened plate, the strains assumed a nonzero value after the buckling point. These nonzero strains then grew monotonically till the point of secondary buckling. Beyond this point it is seen that the bending strains then predictably jumped and assumed negative values. 


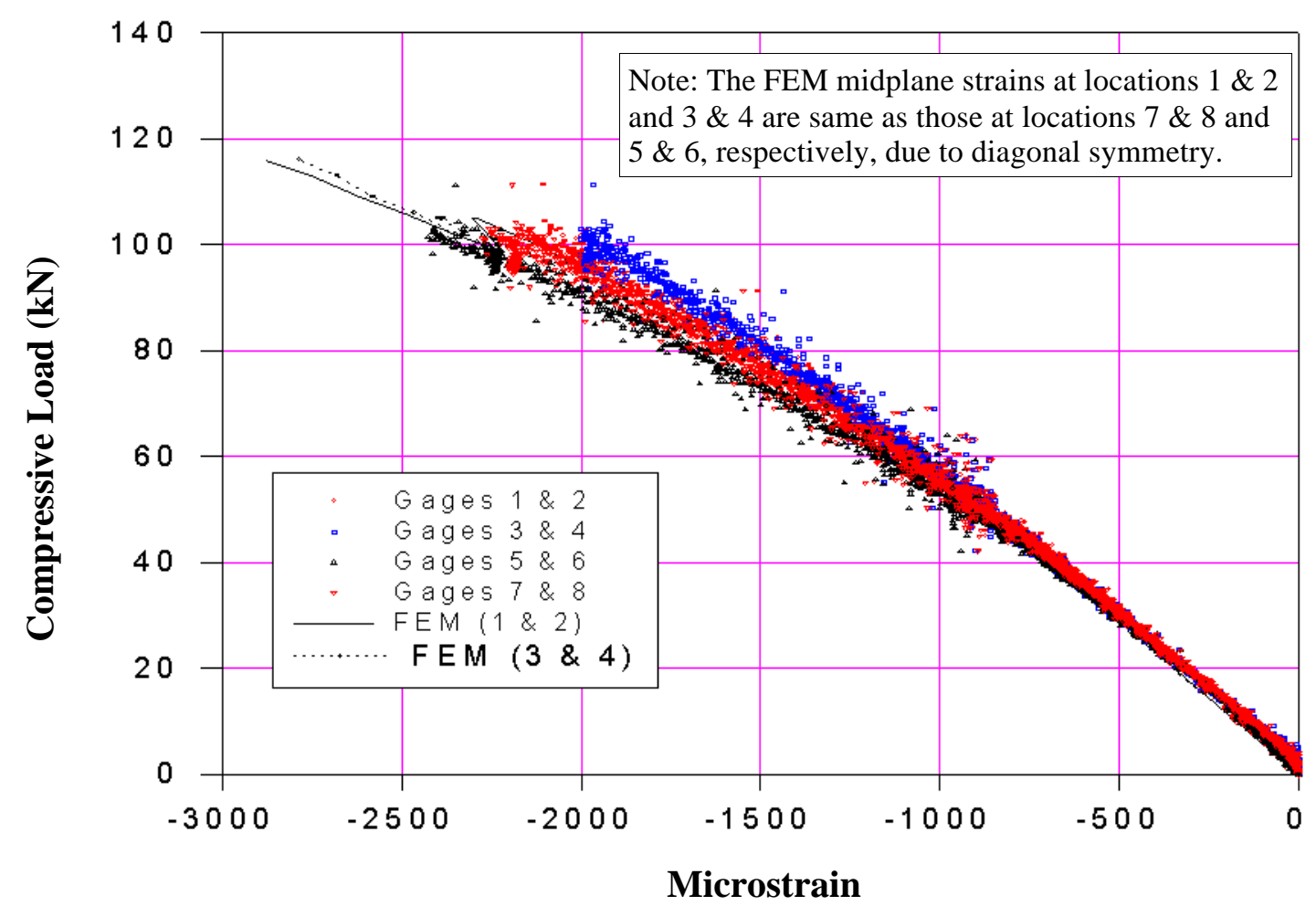

Fig. 5.10: Midplane Strains in Stiffened $[ \pm 45 / 0 / 90]_{S}$ Plate

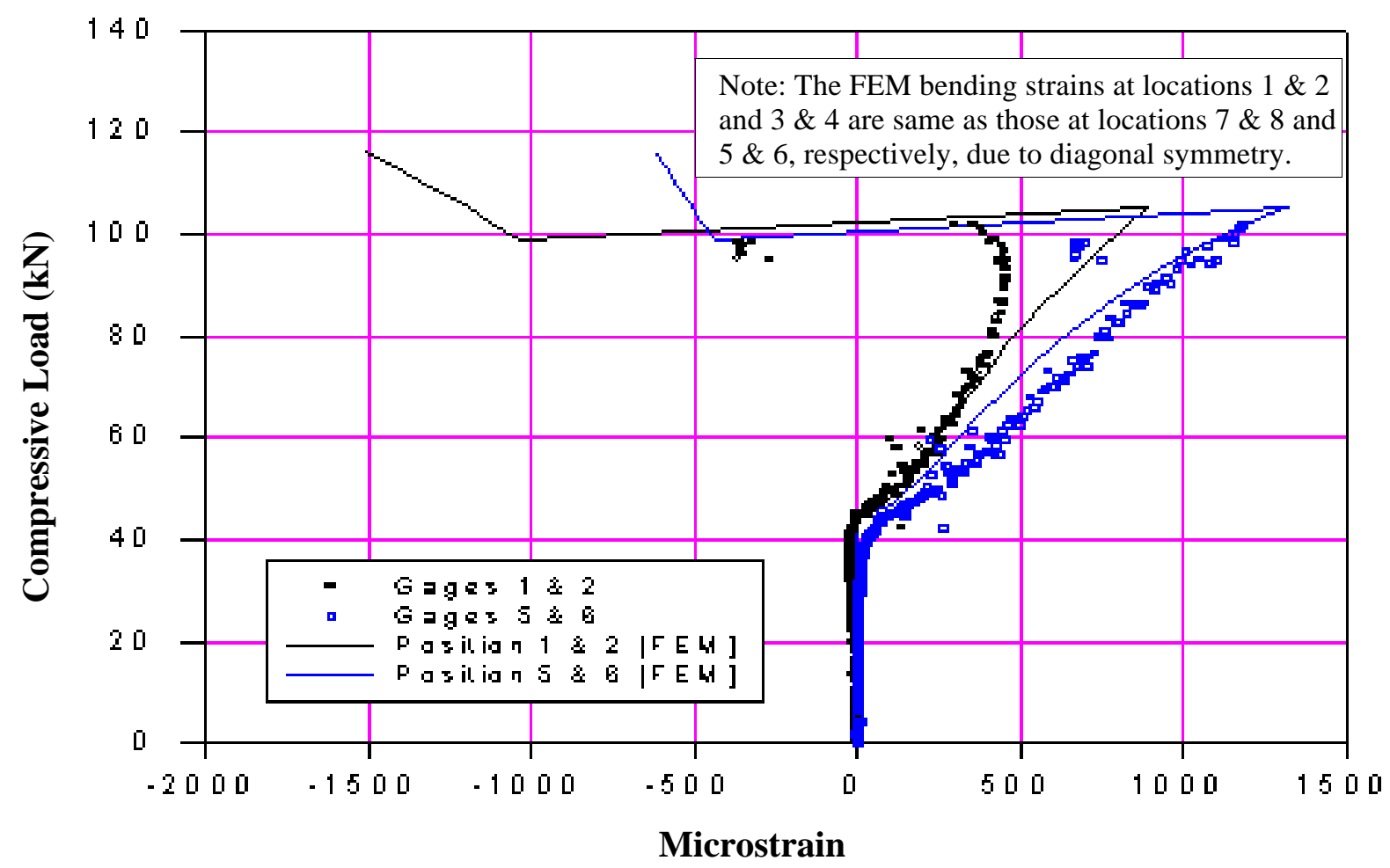

Fig. 5.11: Bending Strains in Top Half of Stiffened $[ \pm 45 / 0 / 90]_{S}$ Plate 


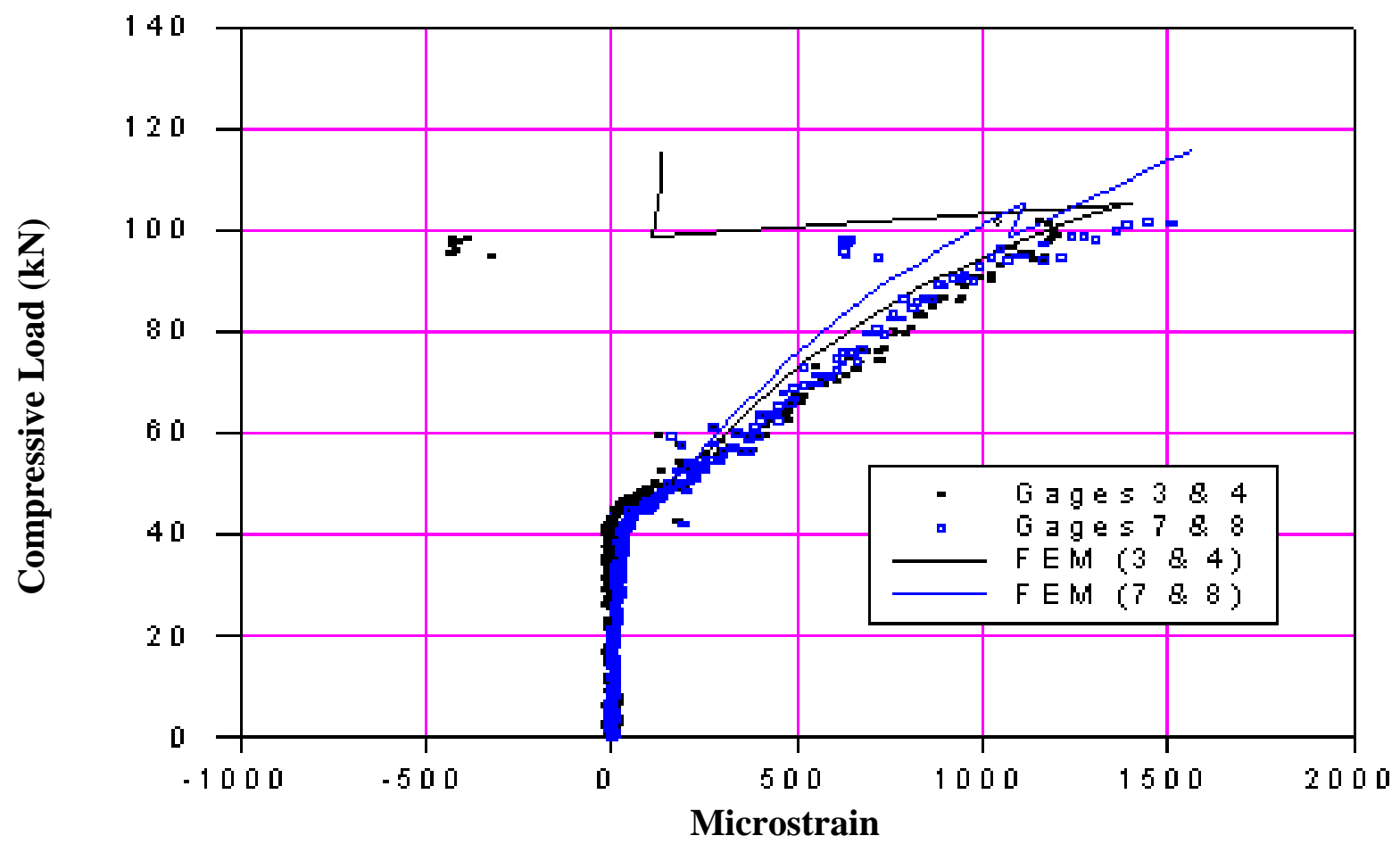

Fig. 5.12: Bending Strains in Bottom Half of Stiffened $[ \pm 45 / 0 / 90]_{S}$ Plate

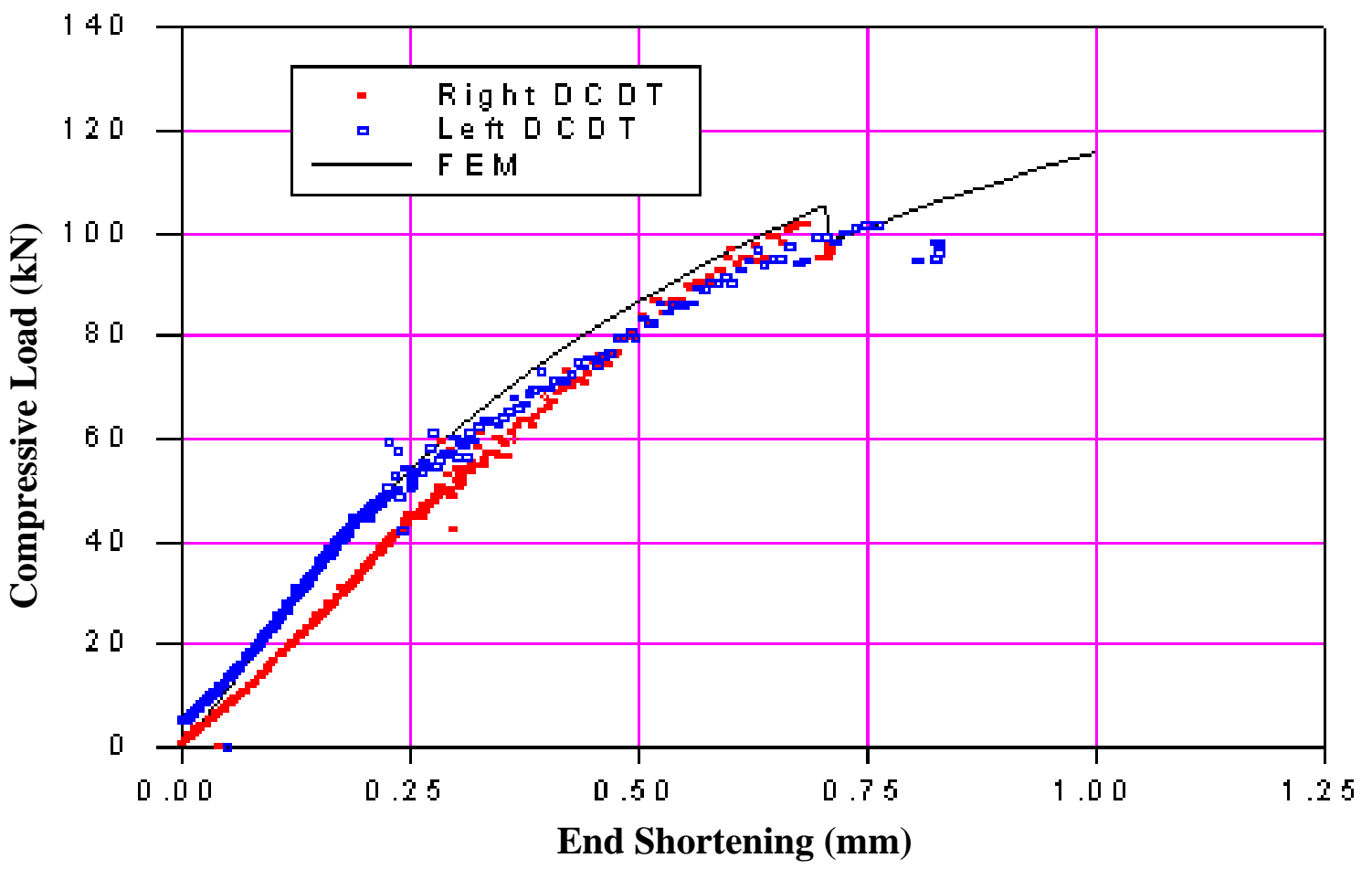

Fig. 5.13: End Shortening Response for Stiffened $[ \pm 45 / 0 / 90]_{S}$ Plate 


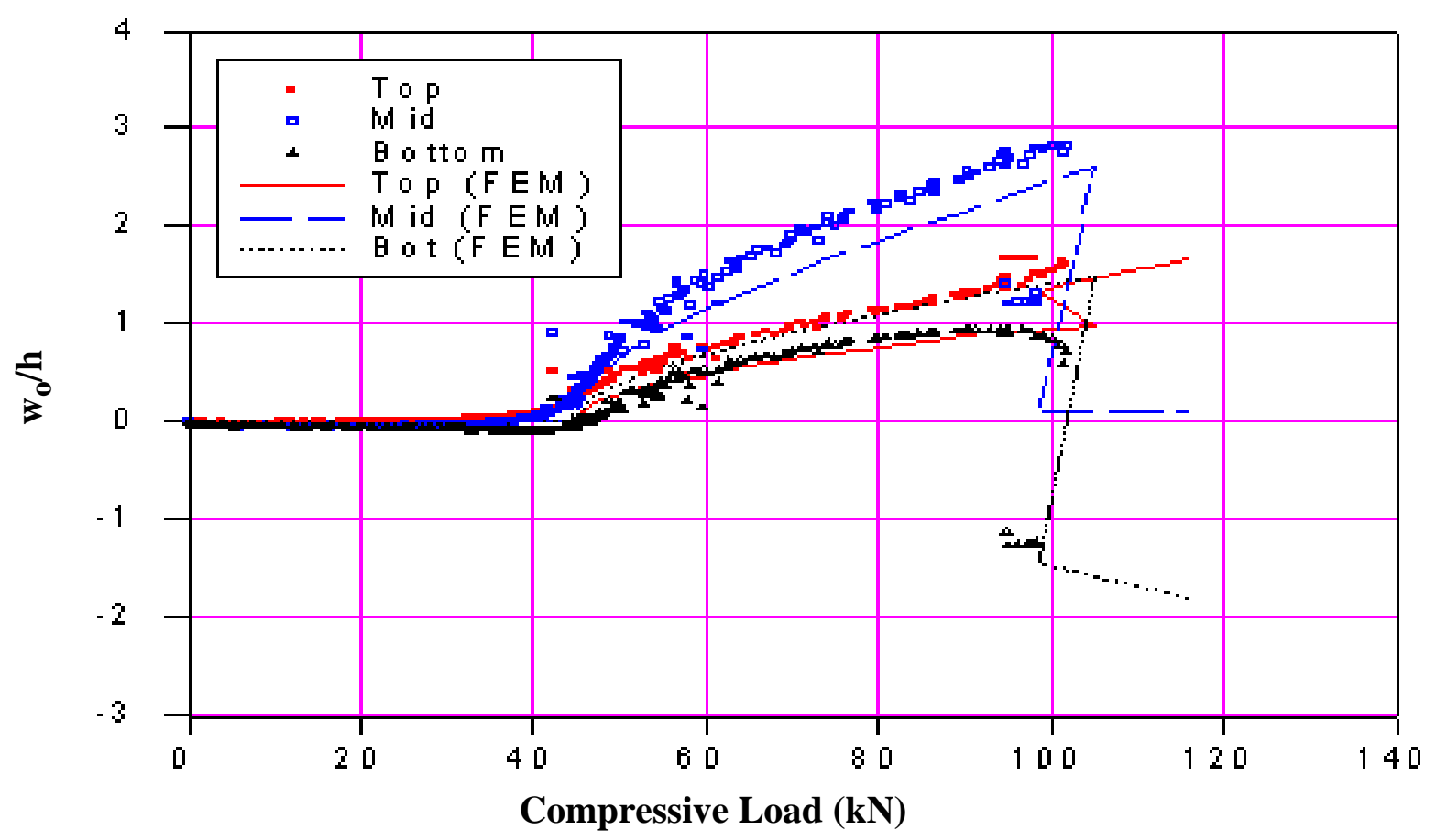

Fig. 5.14: Out-of-Plane Deflection Response for Stiffened $[ \pm 45 / 0 / 90]_{\mathrm{s}}$ Plate
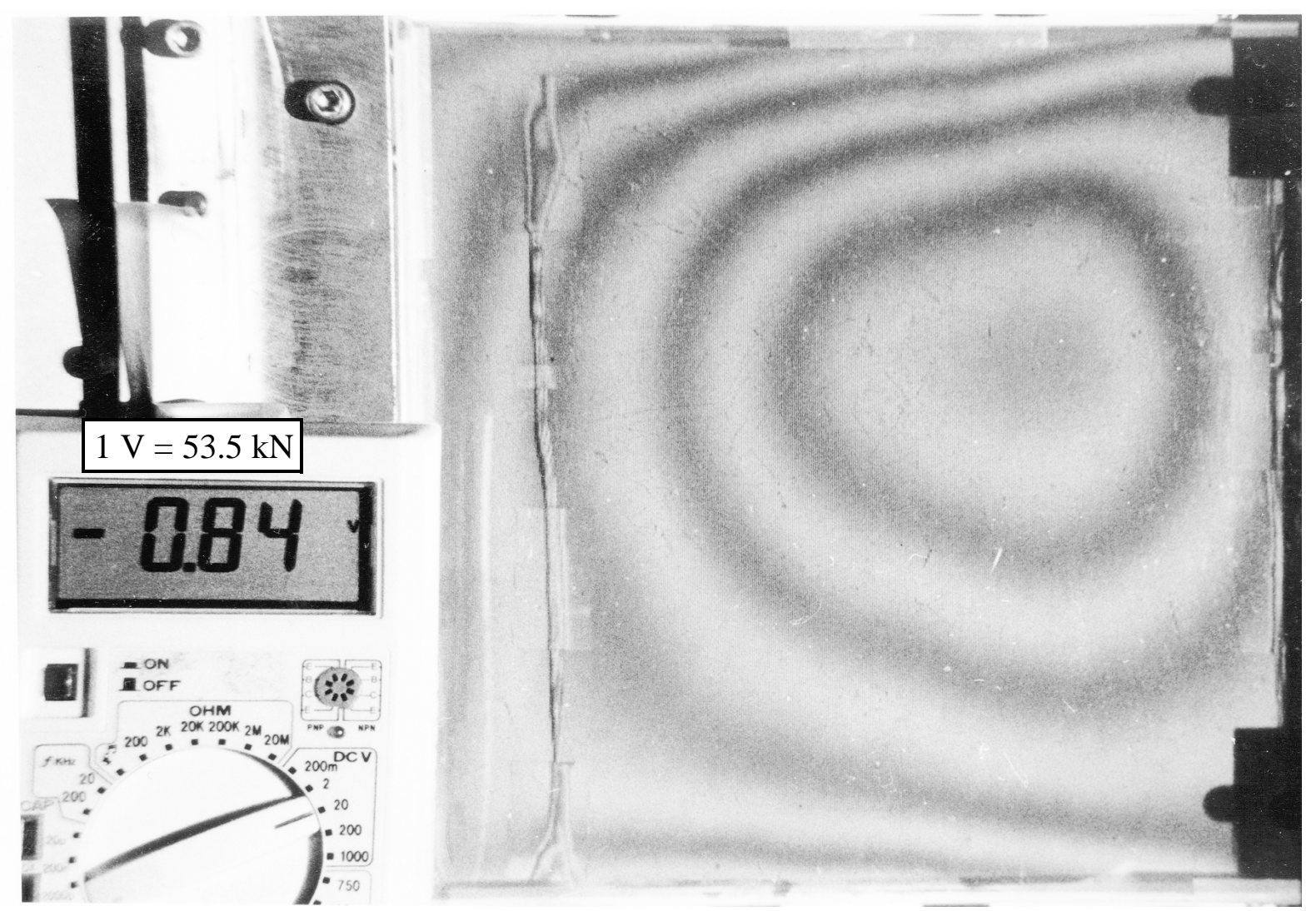

Fig. 5.15: Postbuckling Moire' Fringe Patterns for Out-of-Plane Deflections in Stiffened $[ \pm 45 / 0 / 90]_{\mathrm{s}}$ Plate 


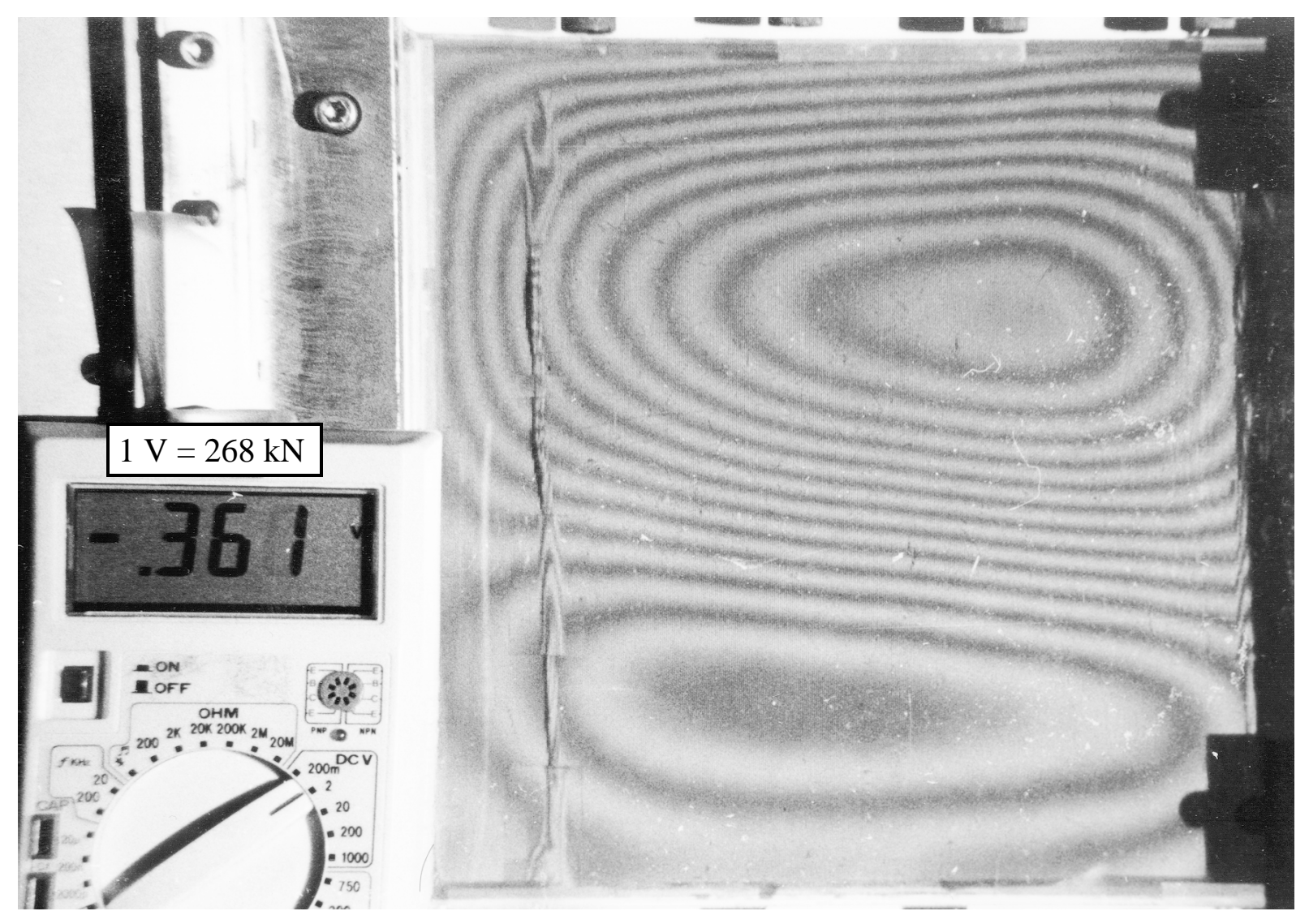

Fig. 5.16: Secondary Buckling Moire' Fringe Patterns for Out-of-Plane Deflections in Stiffened $[ \pm 45 / 0 / 90]_{S}$ Plate

\subsubsection{The $\left[ \pm 45 / 0_{2}\right]_{\mathrm{s}}$ Plates}

Figures 5.17 through 5.26 depict various responses of $\left[ \pm 45 / 0_{2}\right]_{\mathrm{s}}$ plates. Of these, Figs. 5.17 through 5.20 are for the unstiffened plate, while the remaining correspond to the stiffened $\left[ \pm 45 / 0_{2}\right]_{\mathrm{s}}$ plate. These figures depict the midplane strain, bending strain, load end shortening, and out-of-plane deflection responses of the plates under study.

\subsubsection{The $\left[ \pm 45 / 0_{2}\right]_{s}$ Unstiffened Plate}

Figures 5.17 and 5.18 show the midplane and bending strain responses of the plate, respectively. Figures 5.19 and 5.20 show the end shortening and out-of-plane deflection responses of the plate. 


\section{Prebuckling and Buckling}

It is seen from Fig. 5.19 that the loading of the plate was fairly uniform, since the responses of the right and left DCDTs were almost uniform and they were similar for the entire loading range. The prebuckling stiffness of the laminate from Figs. 5.17 and 5.19 was found to be 93.8 $\mathrm{GPa}$ and $87.2 \mathrm{GPa}$, respectively. These values are very close to the numerically evaluated value of $91.6 \mathrm{GPa}$. The buckling point of this plate was found to be $22 \mathrm{kN}$, which is very close to the predicted value of $24 \mathrm{kN}$. The Moire' fringe patterns for the out-of-plane deflection showed that the buckling pattern had one half-wave in both $x$ and $y$ directions. This was consistent with finite element predictions.

\section{Postbuckling}

It is seen in Fig. 5.20 that the out-of-plane deflections increased monotonically with growing inplane compressive loading. Once again, this plate, similar to its quasi-isotropic counterpart, exhibited no secondary buckling. This once again confirmed the finite element predictions. Figure 5.19 shows that numerically evaluated end shortening response of the plate was consistent with experimental observations. This figure, along with Fig. 5.17, which shows the midplane strain response of the plate, shows that the postbuckling stiffness of the plate was significantly less than the prebuckling stiffness. Specifically, the displacement data yielded a postbuckling stiffness of $41.1 \mathrm{GPa}$ and the strain data yielded $43.3 \mathrm{GPa}$. These were slightly higher than the predicted value of $38.8 \mathrm{GPa}$.

Figures 5.17 and 5.18 show that the agreement between predicted values of midplane and bending strains and experimental observations was good. Also, since the values of $D_{16}$ an $D_{26}$ terms in the bending matrix $D$ were large for this laminate, it was expected that the out-of-plane deflection patterns would not be symmetric with respect to the horizontal and vertical planes of geometric symmetry. This was seen in the Moire' fringe patterns, as well as in the behavior of bending strains shown in Fig. 5.18. 


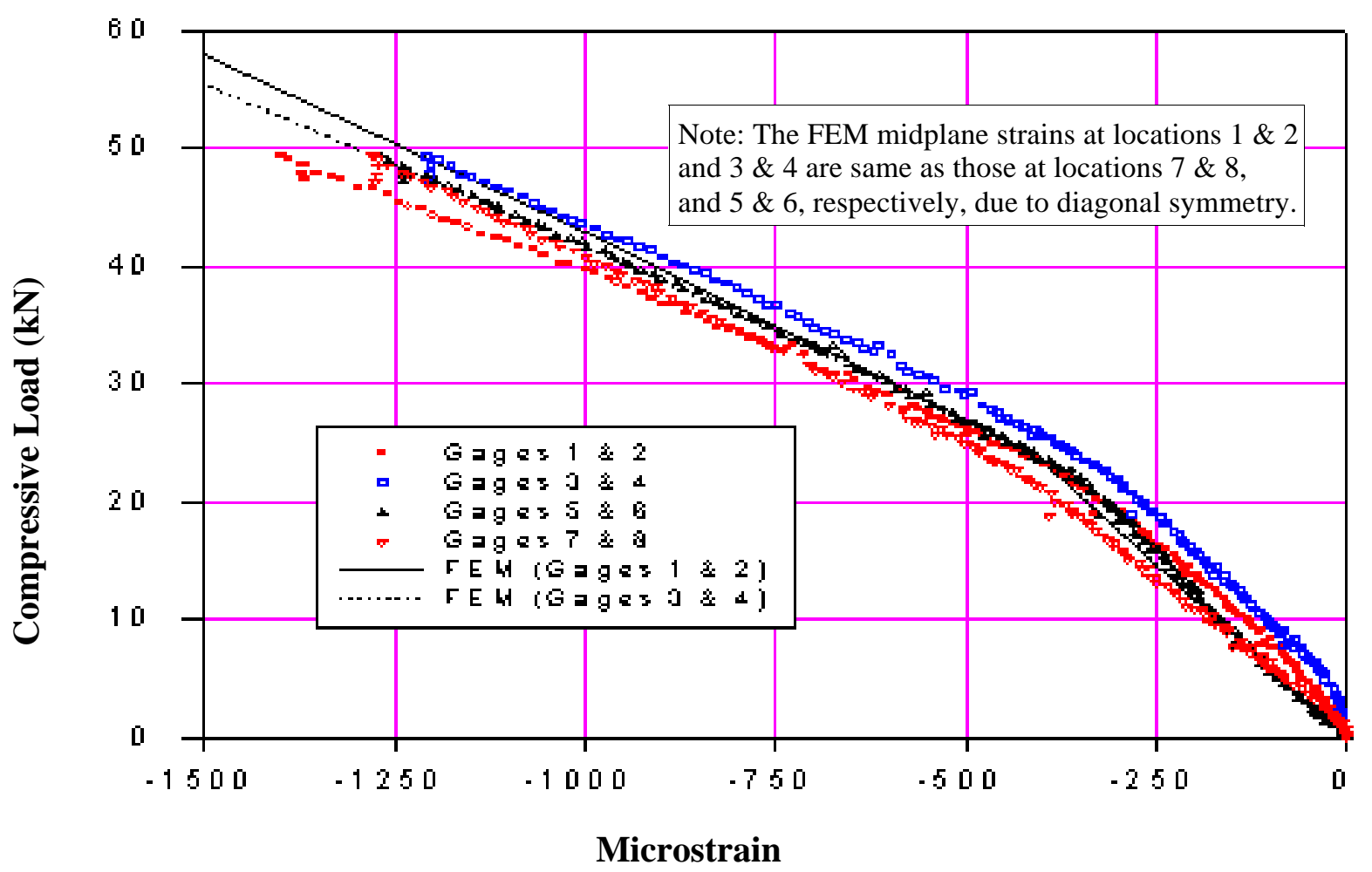

Fig. 5.17: Midplane Strains in Unstiffened $\left[ \pm 45 / 0_{2}\right]_{\mathrm{s}}$ Plate

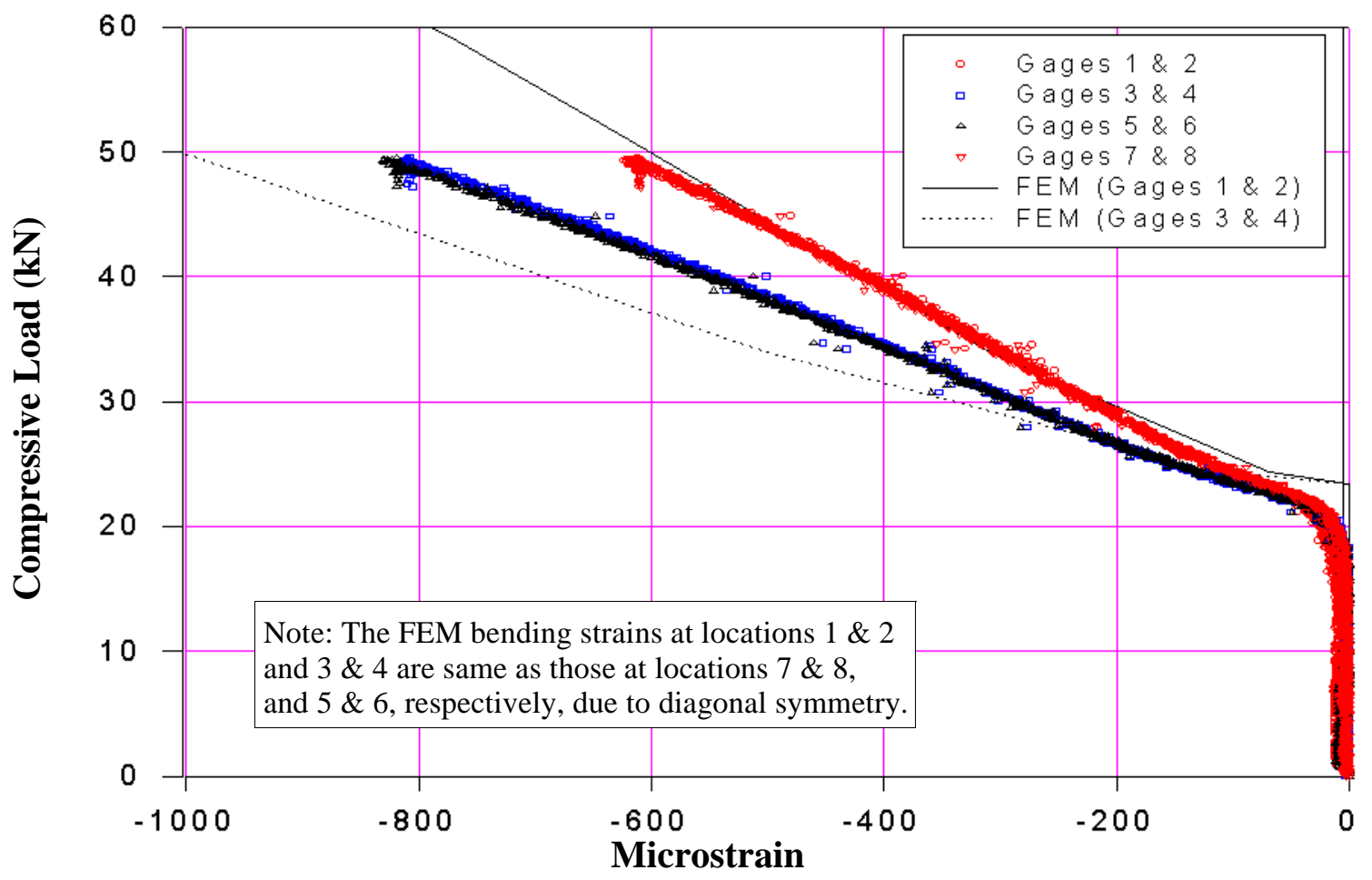

Fig. 5.18: Bending Strains in Unstiffened $\left[ \pm 45 / 0_{2}\right]_{\mathrm{s}}$ Plate 


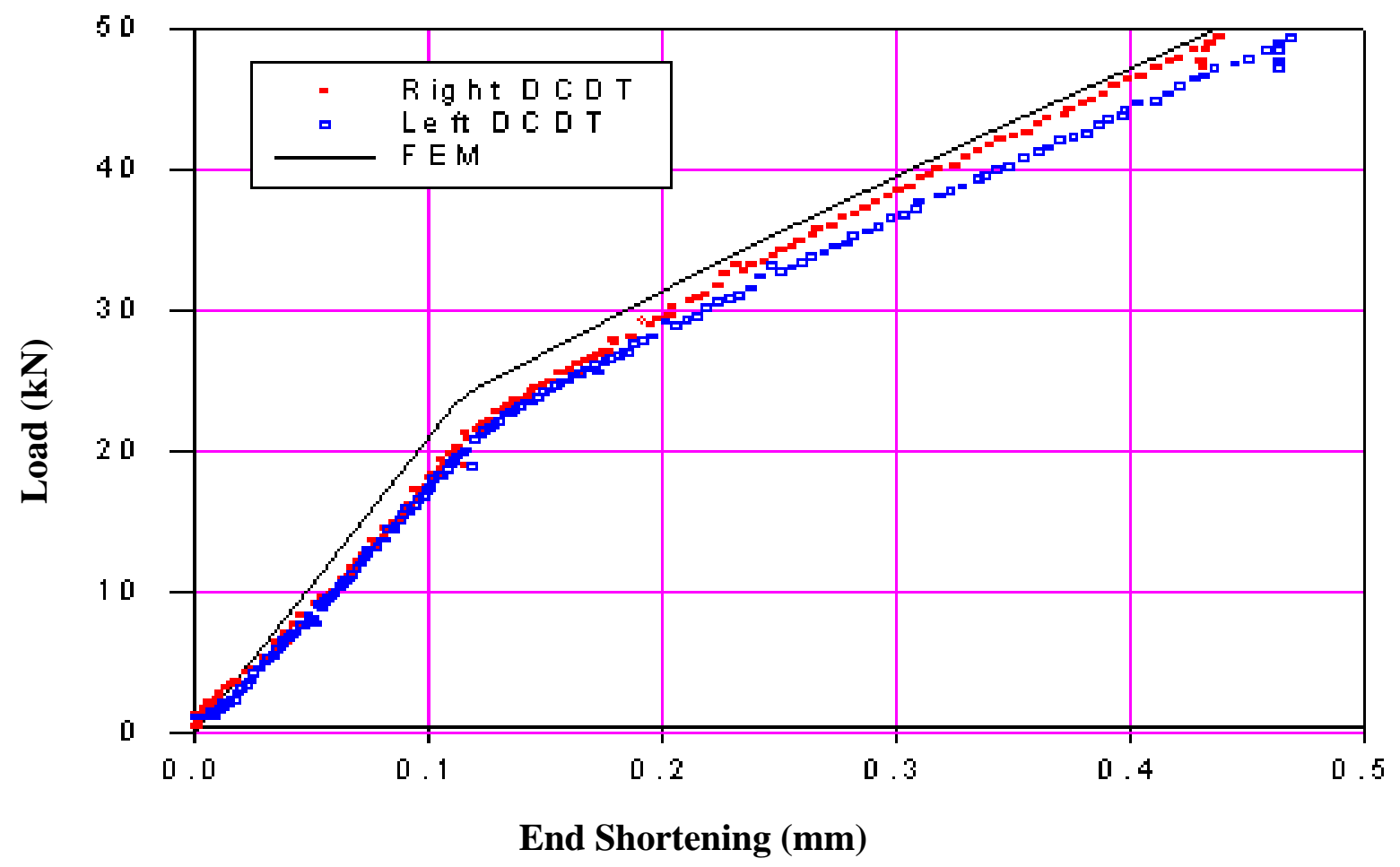

Fig. 5.19: End Shortening Response for Unstiffened $\left[ \pm 45 / 0_{2}\right]_{S}$ Plate

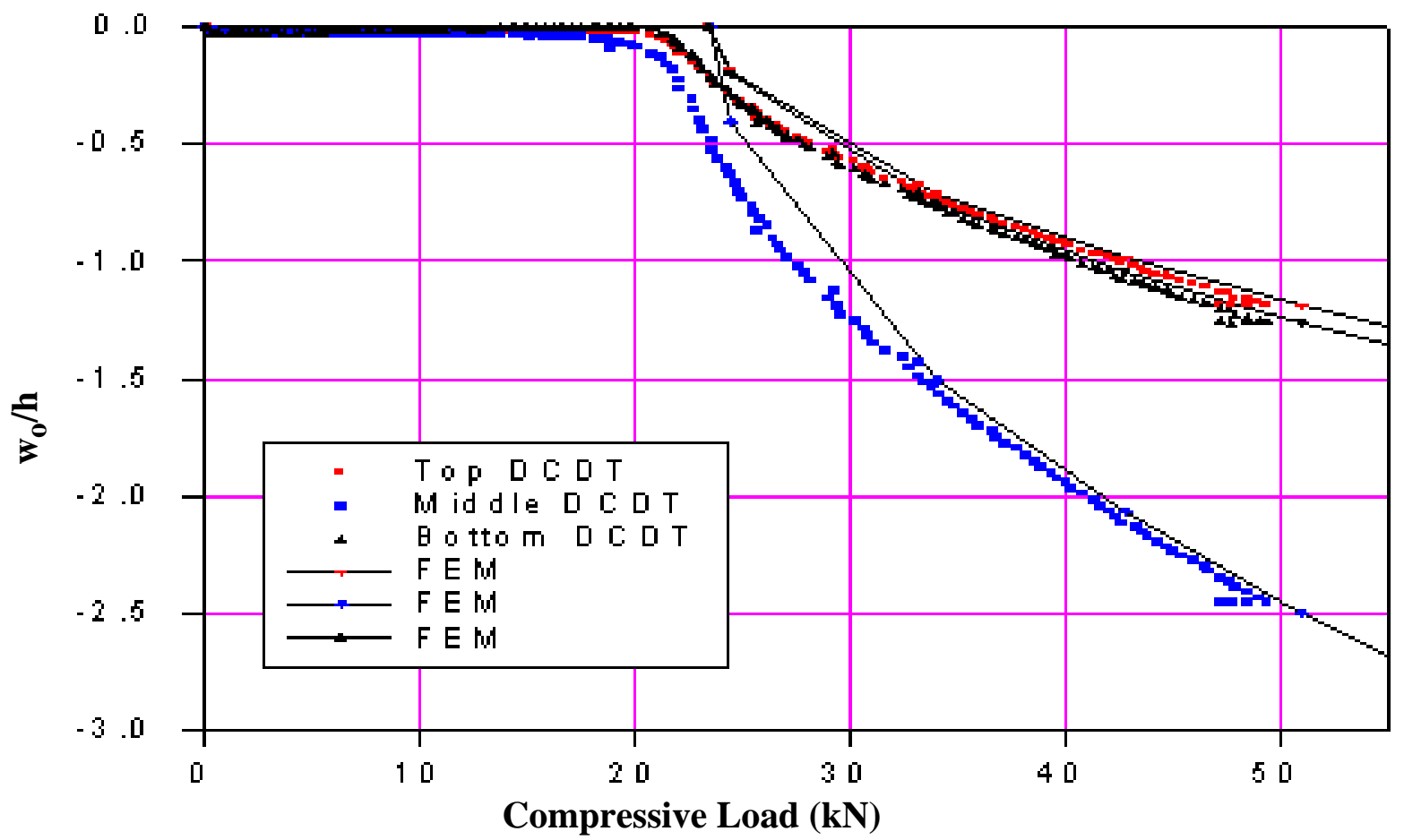

Fig. 5.20: Deflection Response For Unstiffened $\left[ \pm 45 / 0_{2}\right]_{S}$ Plate 


\subsubsection{The $\left[ \pm 45 / 0_{2}\right]_{\mathrm{s}}$ Stiffened Plate}

Figures 5.21 and 5.22 show the midplane and bending strain responses of the stiffened $\left[ \pm 45 / 0_{2}\right]_{\mathrm{S}}$ plate, respectively. Figures 5.23 and 5.24 show the end shortening and out-of-plane deflection responses of the plate. Finally, Figs. 5.25 and 5.26 show the Moire' fringe patterns for out-ofplane deflections for the plate.

\section{Prebuckling and Buckling}

It is seen from Fig. 5.23 that the loading of the plate was fairly uniform, since the responses of the right and left DCDTs were almost uniform and similar for the entire loading range. The prebuckling stiffness of the stiffened plate from Figs. 5.21 and 5.23 was found to be $102 \mathrm{GPa}$ and $88 \mathrm{GPa}$, respectively. The value of prebuckling stiffness as obtained from Fig. 5.21, that is $102 \mathrm{GPa}$, was close to the numerically predicted value of $107 \mathrm{GPa}$.

Figure 5.25 shows the buckling out-of-plane deflections just after the plate had buckled. The load measured at this condition, as also seen in the figure, was found to be equivalent to 0.702 volts, which corresponded to $37.6 \mathrm{kN}$, since each volt at this load level corresponded to $53.5 \mathrm{kN}$. This was close to the numerically predicted value of $40 \mathrm{kN}$. The Moire' fringe patterns for the out-of-plane deflection showed that the buckling pattern had one half-wave in both $x$ and $y$ directions. This was consistent with finite element predictions.

\section{Postbuckling}

As the plate was loaded into the postbuckling range, the amplitude of the deflection patterns increased monotonically with increasing loads, up to a certain extent. This can be seen in Fig. 5.23. However, beyond a certain load level, the plate almost instantly changed its configuration, and developed a shape that had two half-waves in the loading direction and one half-wave in the $y$ direction. The video recording of the experiment revealed that the actual snap-through event occurred when the inplane load applied to the plate was $136 \mathrm{kN}$. The corresponding voltage 
signal is also seen in Fig. 5.26. This value was close to its the predicted value of $130 \mathrm{kN}$. Figure 5.26 also shows the Moire' fringe pattern of the out-of-plane deflections of the plate just after the snap-through event. The load drop associated with this event was experimentally found to be 2.0 $\mathrm{kN}$. This value was evaluated by noticing the drop as recorded by the video camera. The corresponding finite element prediction was $7.0 \mathrm{kN}$. The postbuckling stiffness as measured by the strains was computed to be $74.1 \mathrm{GPa}$ and by the displacements to be $64.0 \mathrm{GPa}$. The finite element predictions yielded $67.7 \mathrm{GPa}$, a value between the two values from the experiments.

The event of secondary buckling can also be seen in Fig. 5.24. It can be seen in the figure that following buckling, the out-of-plane deflections at all three locations of DCDTs gradually rose with increasing load. However, when the external load exceeded secondary buckling load, the deflection at the middle of the plate dropped to a value close to zero. Also, the deflection at the top DCDT location remained positive and that at the bottom DCDT location became negative. The rapid transition in the signs and values of the out-of-plane displacements at these three locations indicated that after buckling in to the secondary configuration the plate had two halfwaves in the loading direction and one half-wave in the transverse direction.

Once again, as in case of other plates discussed so far, the agreement between predicted values of midplane strains, bending strains, out-of plane deflections, and end shortening was found to be reasonable. 


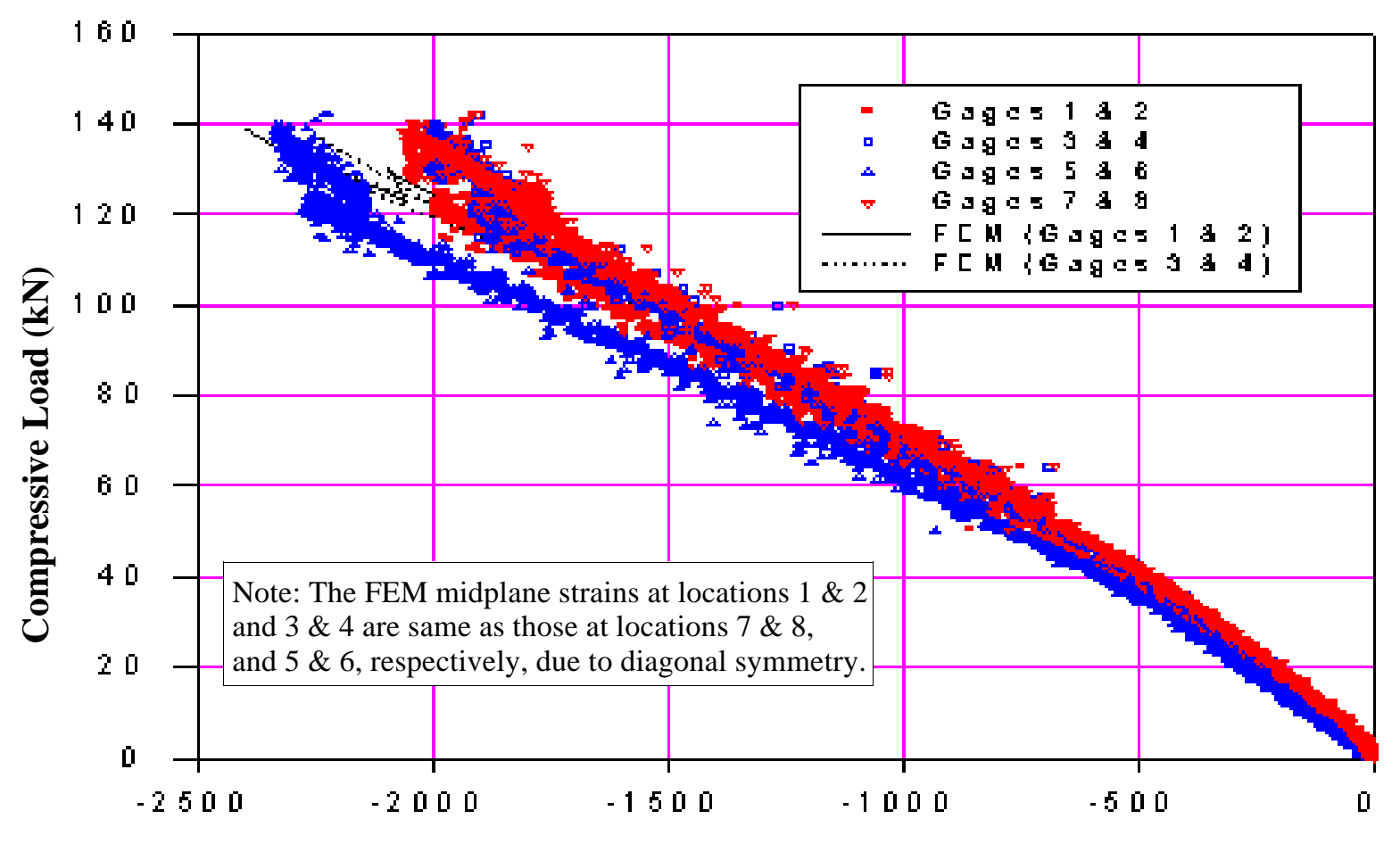

Microstrain

Fig. 5.21: Midplane Strains in Stiffened $\left[ \pm 45 / 0_{2}\right]_{\mathrm{s}}$ Plate

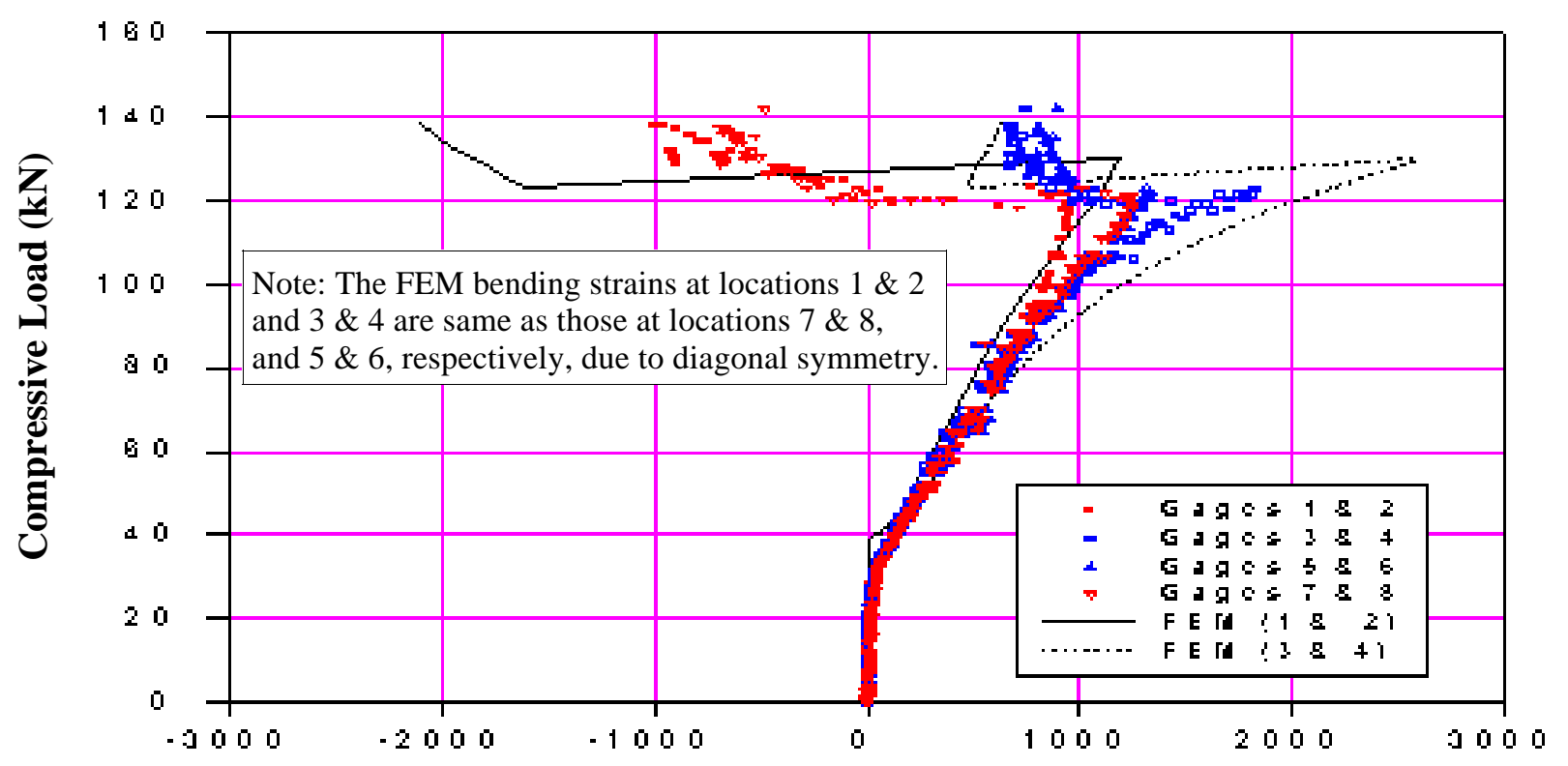

Microstrain

Fig. 5.22: Bending Strains in Top Half of Stiffened $\left[ \pm 45 / 0_{2}\right]_{\mathrm{S}}$ Plate 


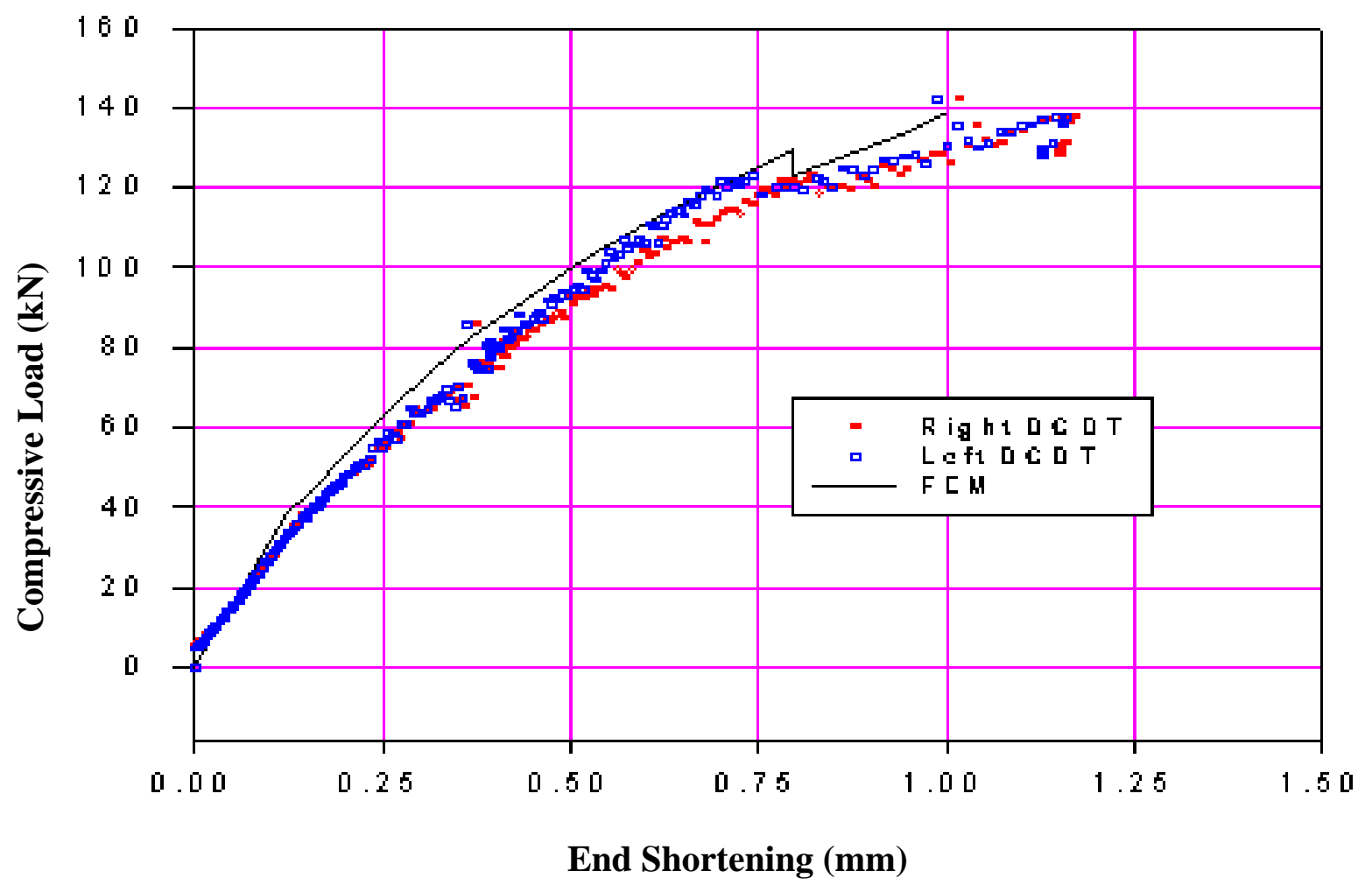

Fig. 5.23: End Shortening Response for Stiffened $\left[ \pm 45 / 0_{2}\right]_{\mathrm{s}}$ Plate

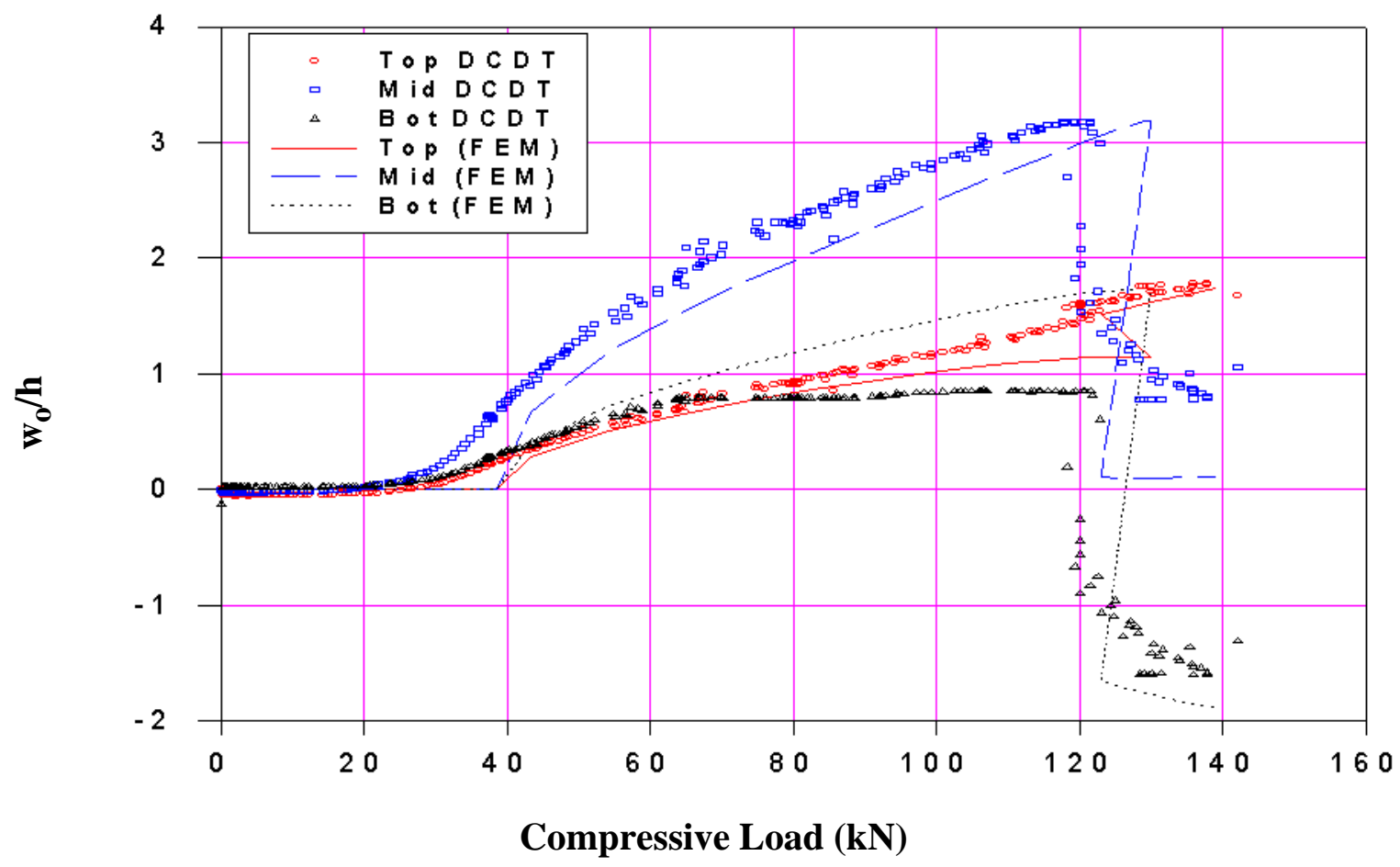

Fig. 5.24: Out-of-Plane Deflection Response for Stiffened $\left[ \pm 45 / 0_{2}\right]_{\mathrm{s}}$ Plate 


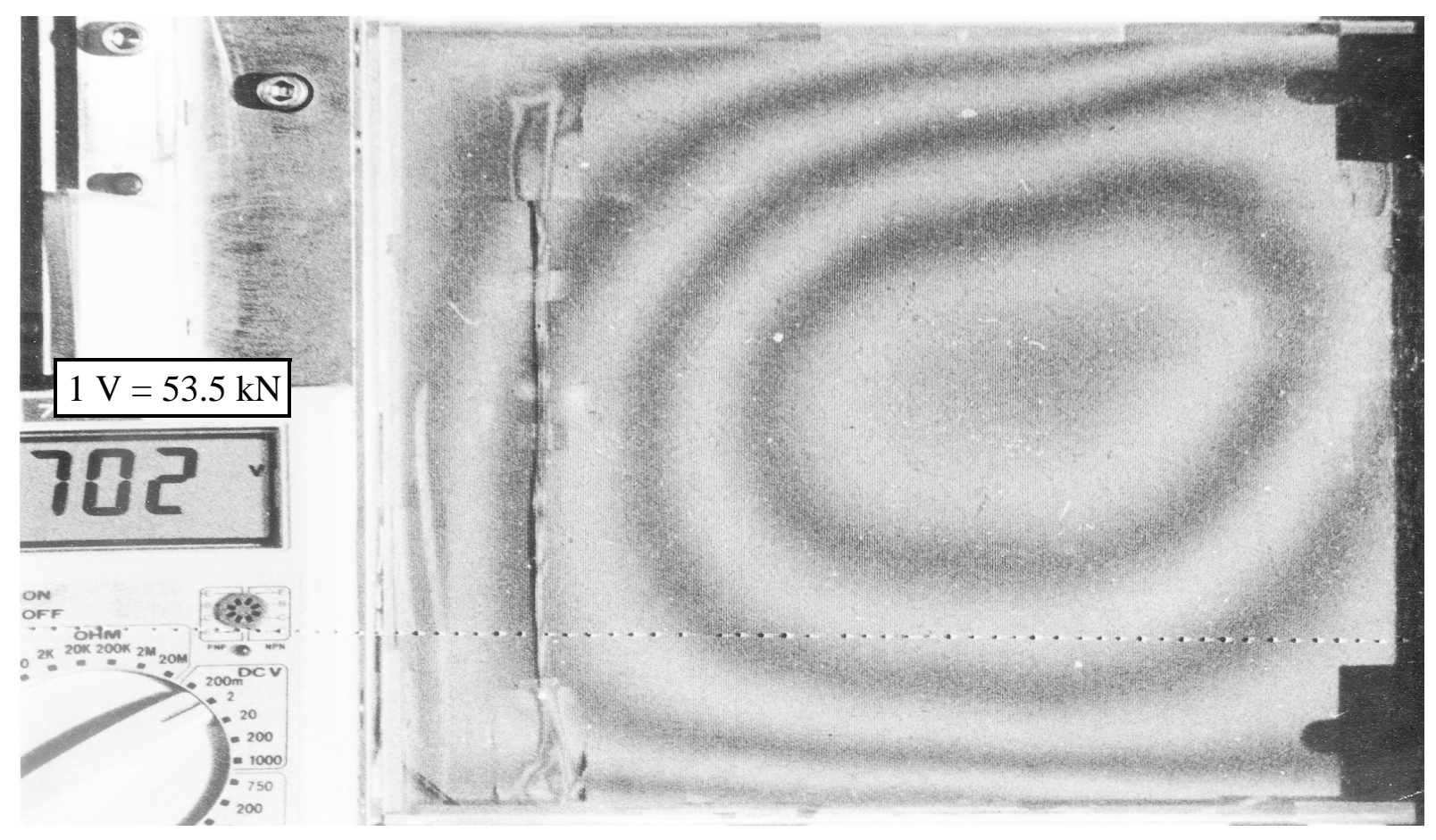

Fig. 5.25: Postbuckling Moire' Fringe Patterns for Out-of-Plane Deflections in Stiffened $\left[ \pm 45 / 0_{2}\right]_{S}$ Plate

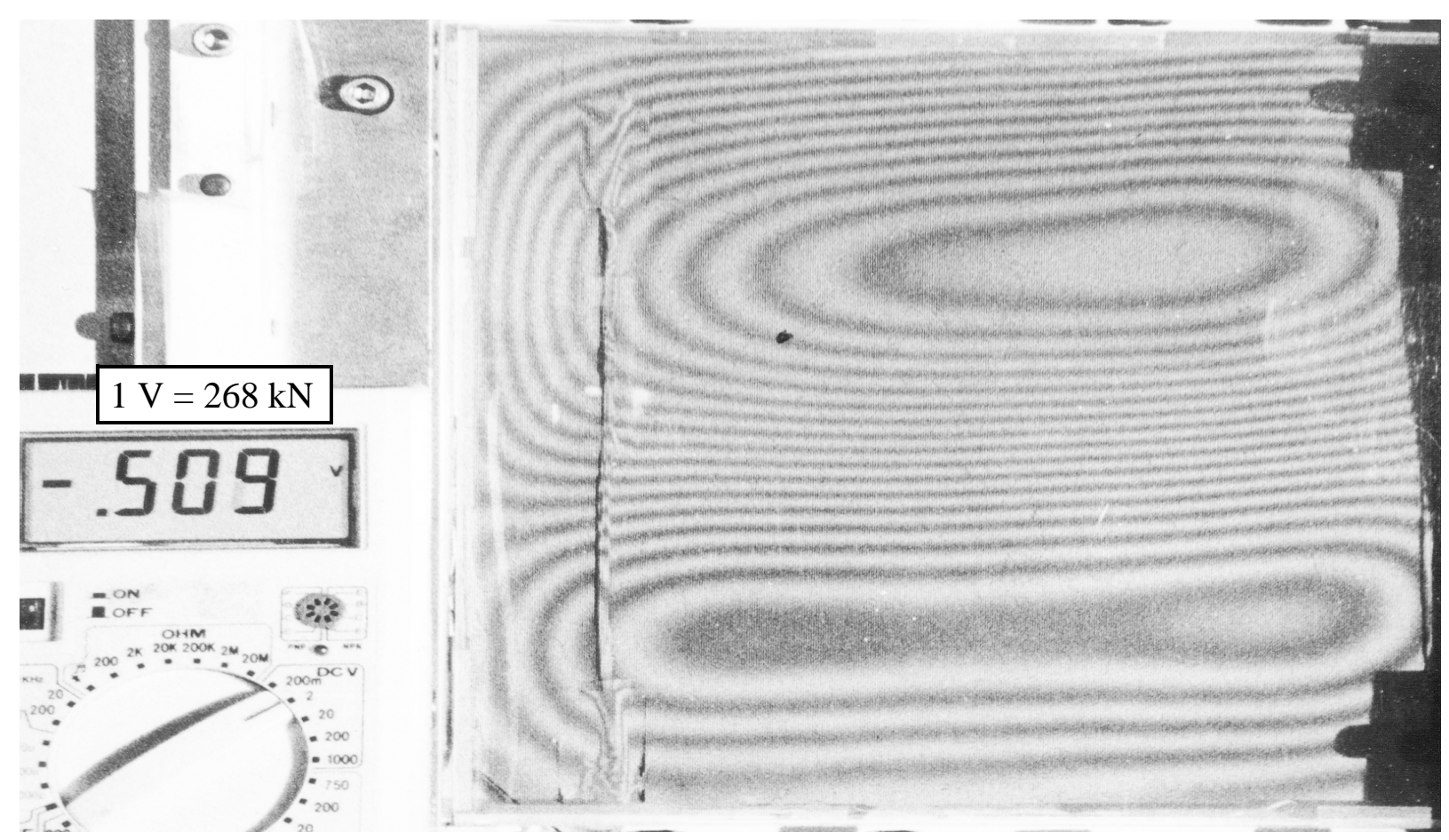

Fig. 5.26: Secondary Buckling Moire' Fringe Patterns for Out-of-Plane Deflections in Stiffened $\left[ \pm 45 / 0_{2}\right]_{S}$ Plate 


\subsubsection{The $\left[ \pm 45 / 90_{2}\right]_{\mathrm{S}}$ Plates}

Figures 5.27 through 5.36 depict the various responses of $\left[ \pm 45 / 0_{2}\right]_{\mathrm{S}}$ plates. Of these, Figs. 5.27 through 5.30 are for the unstiffened plate, while the remaining correspond to the stiffened $\left[ \pm 45 / 90_{2}\right]_{\mathrm{S}}$ plate. These figures depict the midplane strain, bending strain, end shortening, and out-of-plane deflection responses of the plates under study.

\subsubsection{The $\left[ \pm 45 / 90_{2}\right]_{\mathrm{S}}$ Unstiffened Plate}

Figures 5.27 and 5.28 show the midplane and bending strain responses of the plate, respectively. Figures 5.29 and 5.30 show the end shortening and out-of-plane deflection responses of the plate.

\section{Prebuckling and Buckling}

It is seen from Fig. 5.29 that the loading of the plate was fairly uniform, since the responses of the right and left DCDTs were almost uniform and they were similar for the entire loading range. The prebuckling stiffness of the laminate from Figs. 5.27 and 5.29 was found to be 23.5 and $24.4 \mathrm{GPa}$, respectively, which were very close to the numerically evaluated value of 24.9 GPa. The buckling point of this plate was found to be $21 \mathrm{kN}$ which was very close to the predicted value of $20.8 \mathrm{kN}$. The Moire' fringe patterns for the out-of-plane deflection showed that the buckling pattern had one half-wave in both $x$ and $y$ directions. This was consistent with finite element predictions.

\section{Postbuckling}

It is seen in Fig. 5.30 that the out-of-plane deflections increased monotonically with increasing compression. Once again, this plate, similar to its quasi-isotropic counterpart, exhibited no secondary buckling. This once again confirmed the finite element predictions. Figure 5.29 shows that numerically evaluated end shortening response of the plate was consistent with experimental observations. This figure, along with Fig. 5.27, which shows the midplane 
response of the plate shows that the postbuckling stiffness of the plate was significantly less than the prebuckling stiffness. In particular, the displacement data resulted in a postbuckling stiffness of $18.8 \mathrm{GPa}$, while the strain data yielded a stiffness of $13.8 \mathrm{GPa}$. The predicted value was 15.1 GPa.

Figures 5.27 and 5.28 show that the agreement between predicted values of midplane and bending strains and experimental observations was good. Also, since the values of $D_{16}$ an $D_{26}$ terms in the bending matrix $D$ were large for this laminate, it was expected that the out-of-plane deflection patterns would not be symmetric with respect to the horizontal and vertical planes of geometric symmetry. This was seen in the Moire' fringe patterns, as well as in the behavior of bending strains, as shown in Fig. 5.28. 


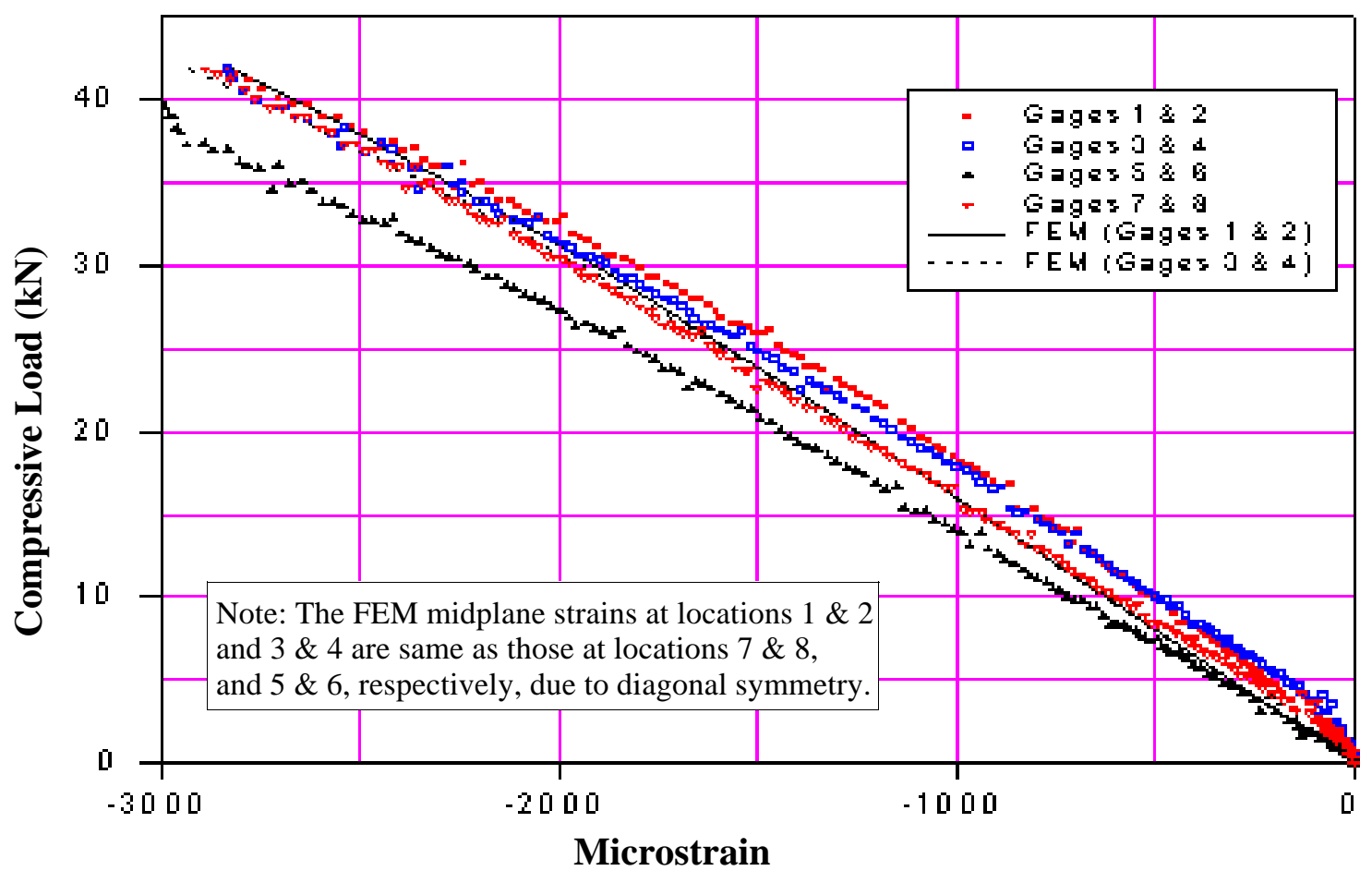

Fig. 5.27: Midplane Strains in Unstiffened $\left[ \pm 45 / 90_{2}\right]_{\mathrm{s}}$ Plate

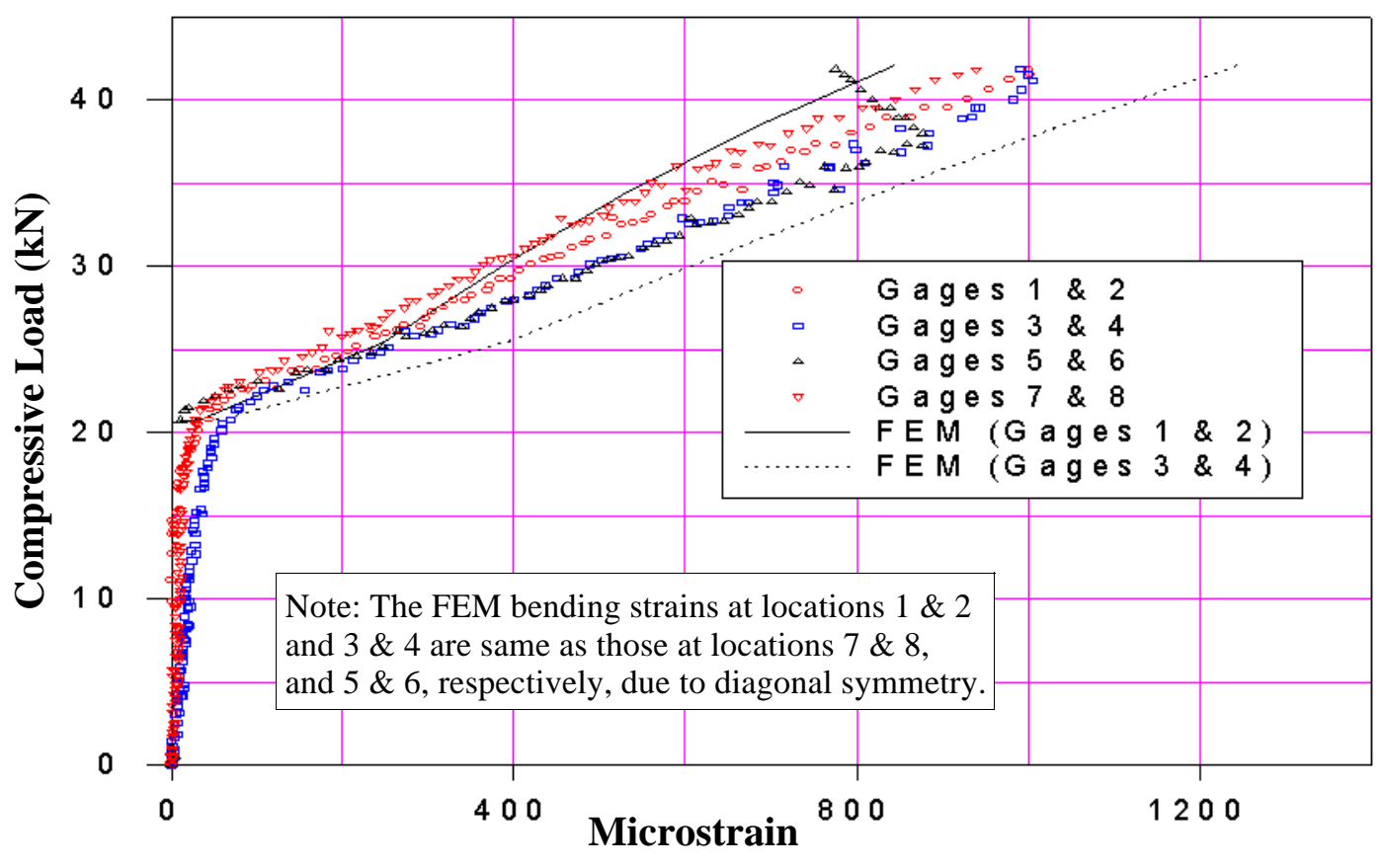

Fig. 5.28: Bending Strains in Unstiffened $\left[ \pm 45 / 90_{2}\right]_{\mathrm{S}}$ Plate 


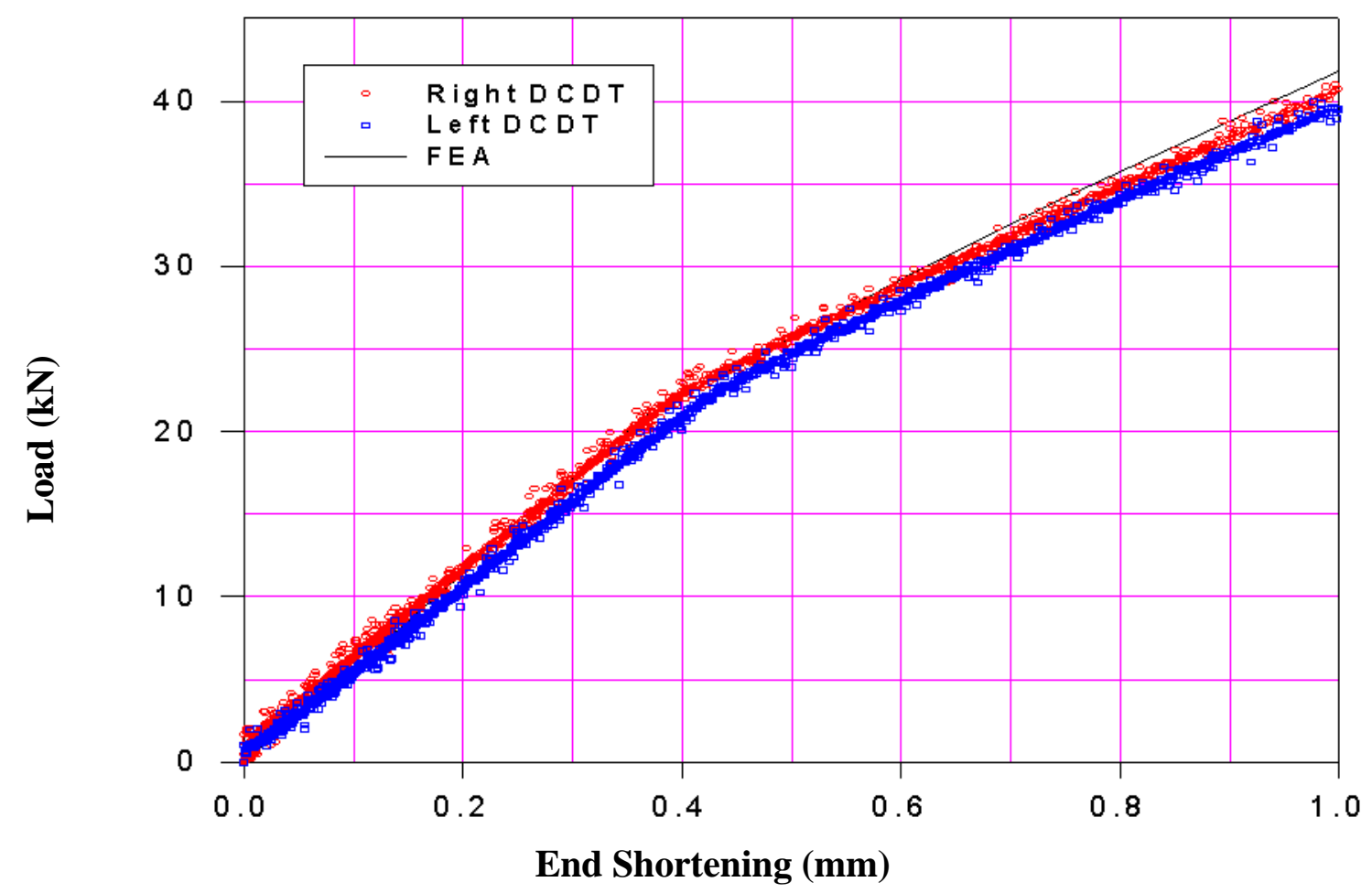

Fig. 5.29: End Shortening Response for Unstiffened $\left[ \pm 45 / 90_{2}\right]_{s}$ Plate

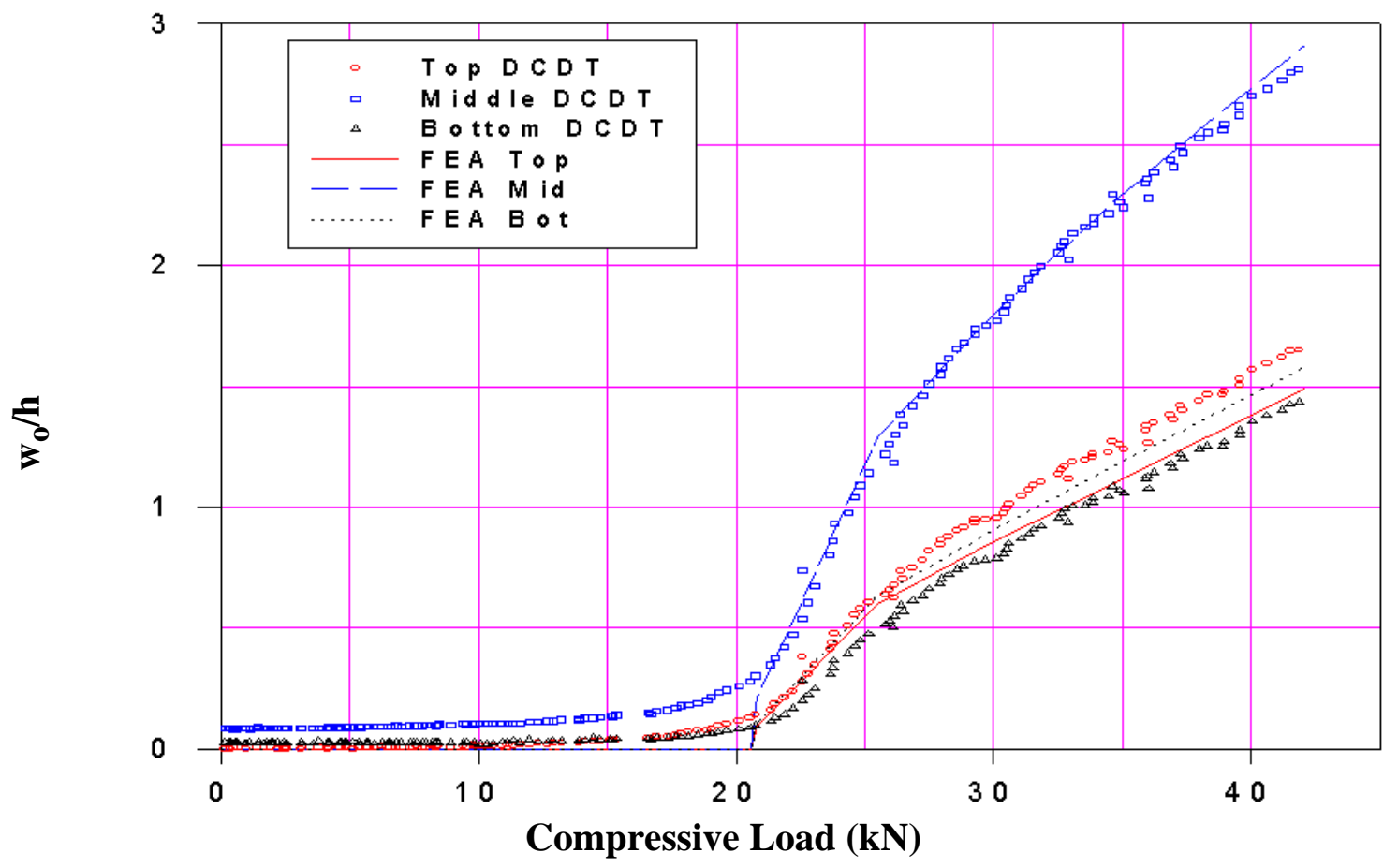

Fig. 5.30: Deflection Response For Unstiffened $\left[ \pm 45 / 90_{2}\right]_{s}$ Plate 


\subsubsection{The $\left[ \pm 45 / 90_{2}\right]_{\mathrm{s}}$ Stiffened Plate}

Figures 5.31 and 5.32 show the midplane and bending strain responses of the stiffened $\left[ \pm 45 / 90_{2}\right]_{\mathrm{S}}$ plate, respectively. Figures 5.33 and 5.34 show the end shortening and out-of-plane deflection responses of the plate. Finally, Figs. 5.35 and 5.36 show the Moire' fringe patterns for out-of-plane deflections for the plate.

\section{Prebuckling and Buckling}

It is seen from Fig. 5.33 that the loading of the plate was fairly uniform, since the responses of the right and left DCDTs were almost uniform and they were similar for the entire loading range. The prebuckling stiffness of the stiffened plate from Figs. 5.31 and 5.33 was found to be 40.4 GPa and 45.0 GPa, respectively, which were very close to the numerically evaluated value of $45.5 \mathrm{GPa}$.

In addition, the buckling load for the plate was experimentally determined to be approximately $60 \mathrm{kN}$, which was very close to the numerically predicted value of $58.6 \mathrm{kN}$. The Moire' fringe patterns for the out-of-plane deflection showed that the buckling pattern had one half-wave in both $x$ and $y$ directions. This was consistent with finite element predictions.

\section{Postbuckling}

As the plate was loaded into postbuckling range, the amplitude of the deflection patterns increased monotonically with increasing loads up to a certain extent. This can be seen in Fig. 5.34. However, beyond a certain load level, the plate almost instantly changed its configuration, and developed a shape that had two half-waves in the loading direction and one half-wave in the $y$ direction. The video recording of the experiment revealed that the actual secondary buckling event occurred when the inplane load applied to the plate was $79 \mathrm{kN}$. The corresponding voltage signal is seen in Fig. 5.36. The numerically predicted value for the occurrence of secondary buckling for this plate was $68.4 \mathrm{kN}$. Also, the load drop associated with the event of secondary 
buckling was experimentally found to be $1.1 \mathrm{kN}$, while the corresponding numerical prediction was $5.1 \mathrm{kN}$. The postbuckling slope computed from the displacement data was $35.5 \mathrm{GPa}$, and that from strain data was $36.2 \mathrm{GPa}$. The predicted postbuckling stiffness was $28.2 \mathrm{GPa}$, a somewhat lower value.

The event of secondary buckling can also be seen in Fig. 5.34. It can be seen in the figure that following buckling, the out-of-plane deflections at all three locations of DCDTs gradually rose with increasing load. However, when the external load exceeded secondary buckling load, the deflection at the middle of the plate dropped to a value close to zero. Also, the deflection at the top DCDT location remains positive and that at the bottom DCDT location became negative. The rapid transition in the signs and values of the out-of-plane displacements at these three locations indicated that after buckling in to the secondary configuration the plate had two half-waves in the loading direction and one half-wave in the transverse direction. 


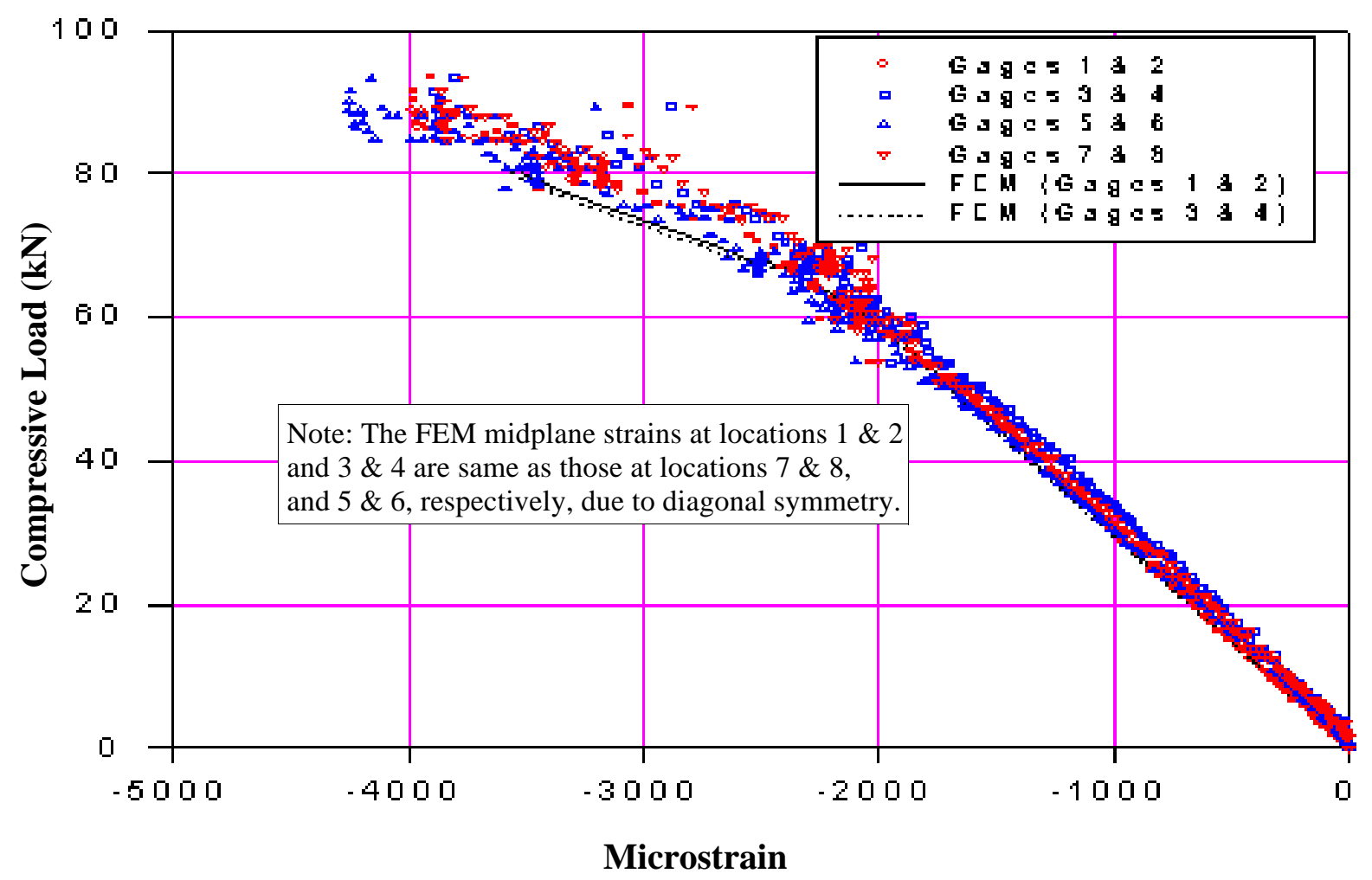

Fig. 5.31: Midplane Strains in Stiffened $\left[ \pm 45 / 90_{2}\right]_{\text {s }}$ Plate

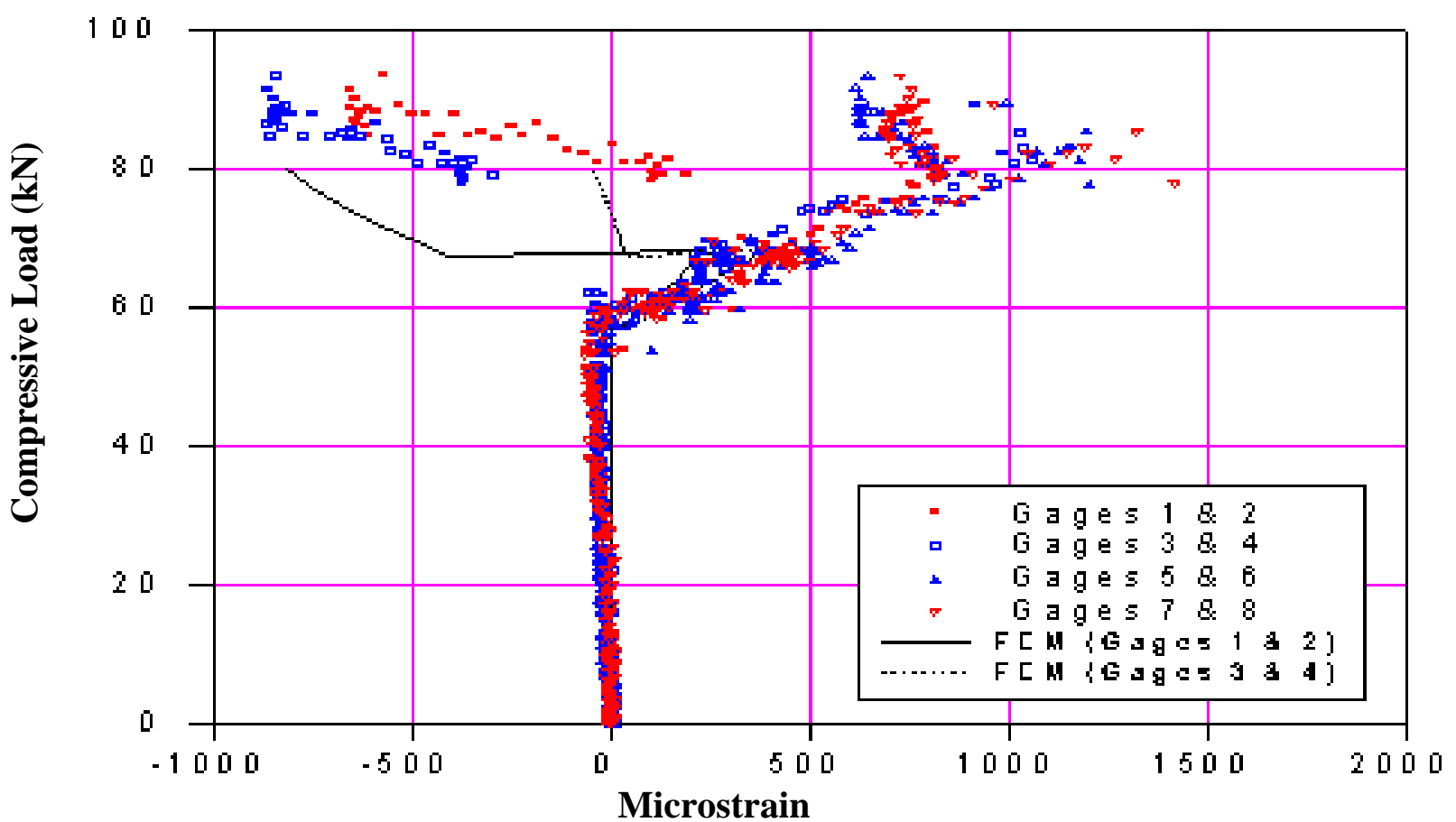

Fig. 5.32: Bending Strains in Stiffened $\left[ \pm 45 / 90_{2}\right]_{\mathrm{s}}$ Plate 


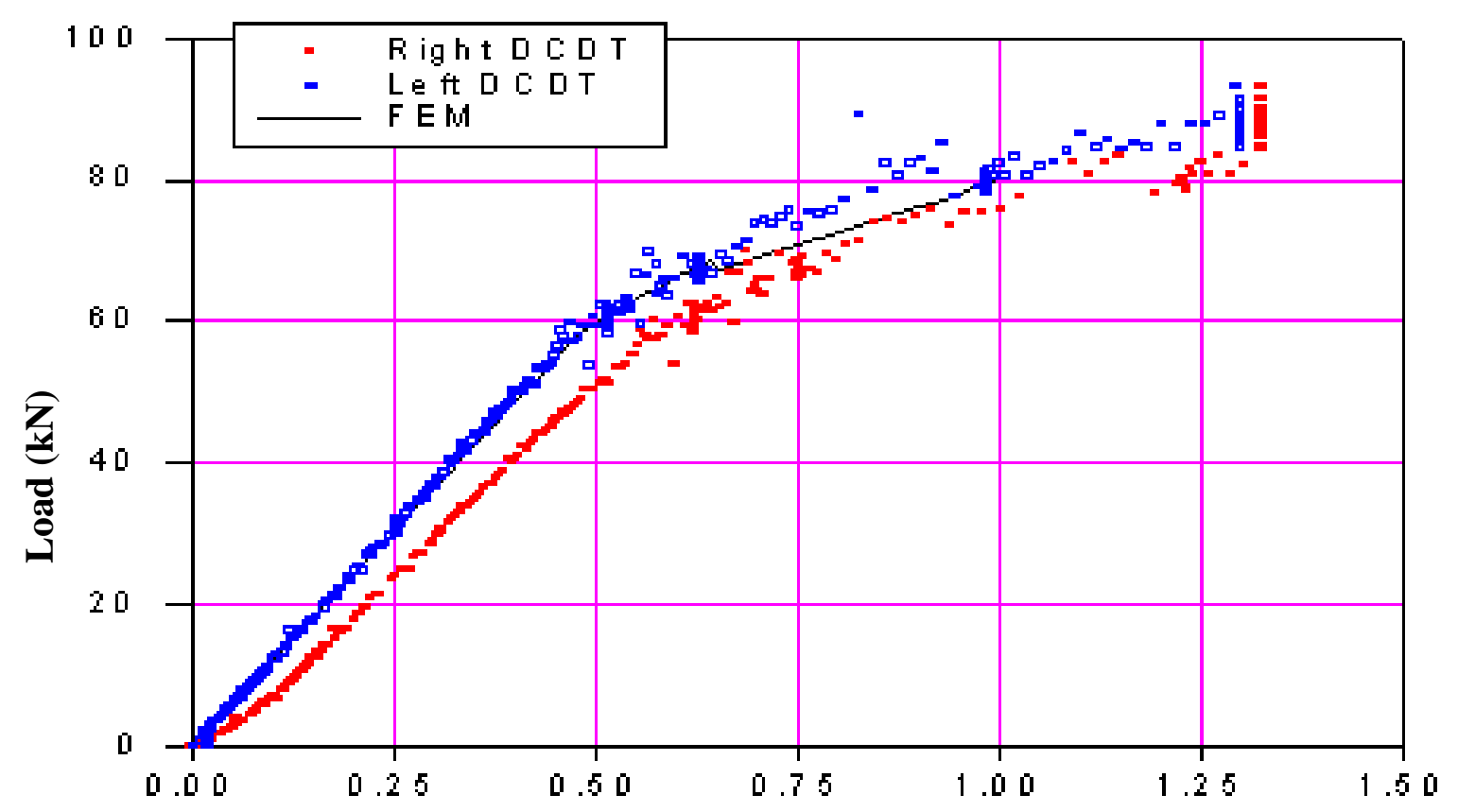

End Shortening (mm)

Fig. 5.33: End Shortening Response for Stiffened $\left[ \pm 45 / 90_{2}\right]_{S}$ Plate

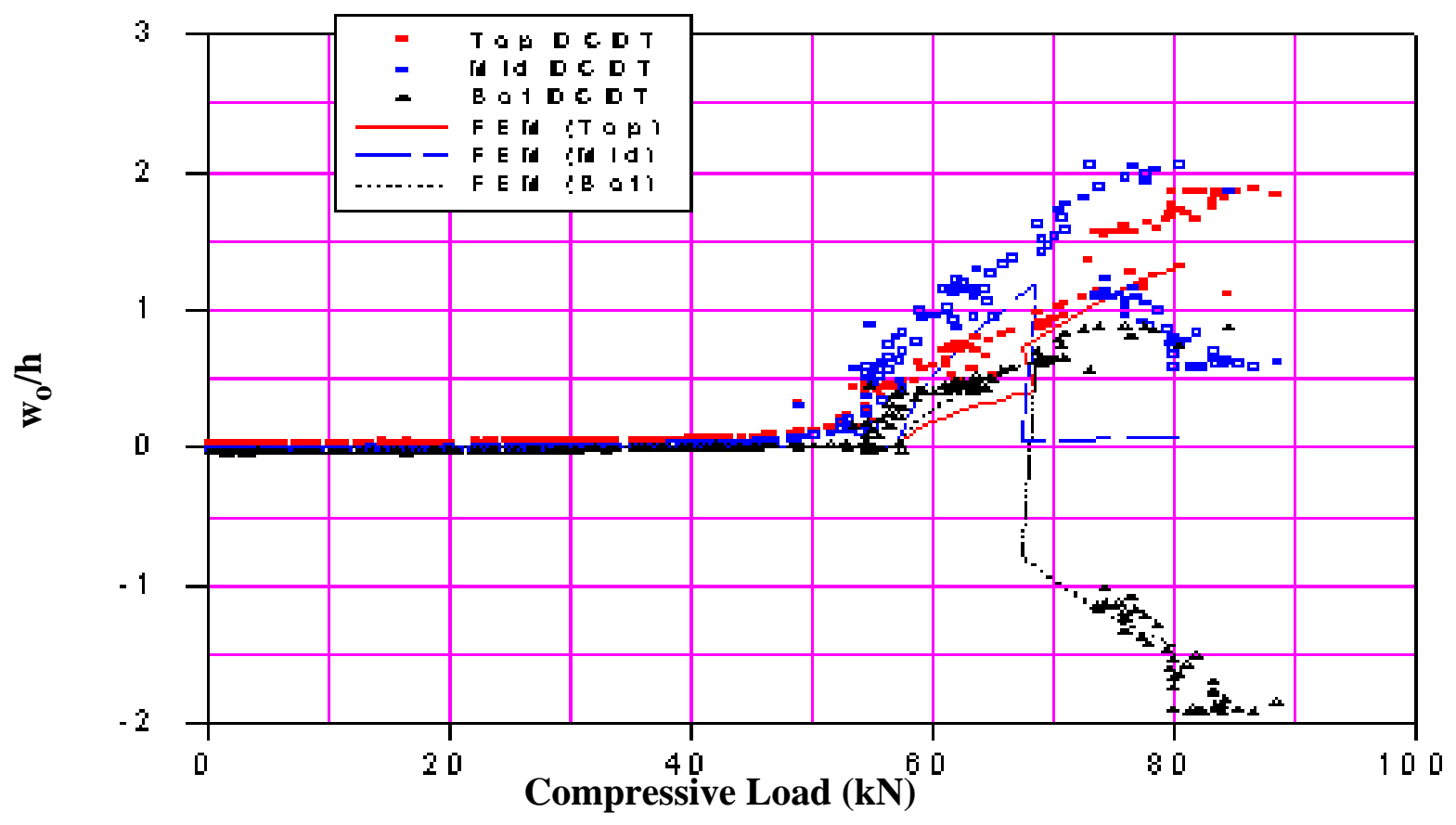

Fig. 5.34: Deflection Response For Stiffened $\left[ \pm 45 / 90_{2}\right]_{\mathrm{s}}$ Plate 


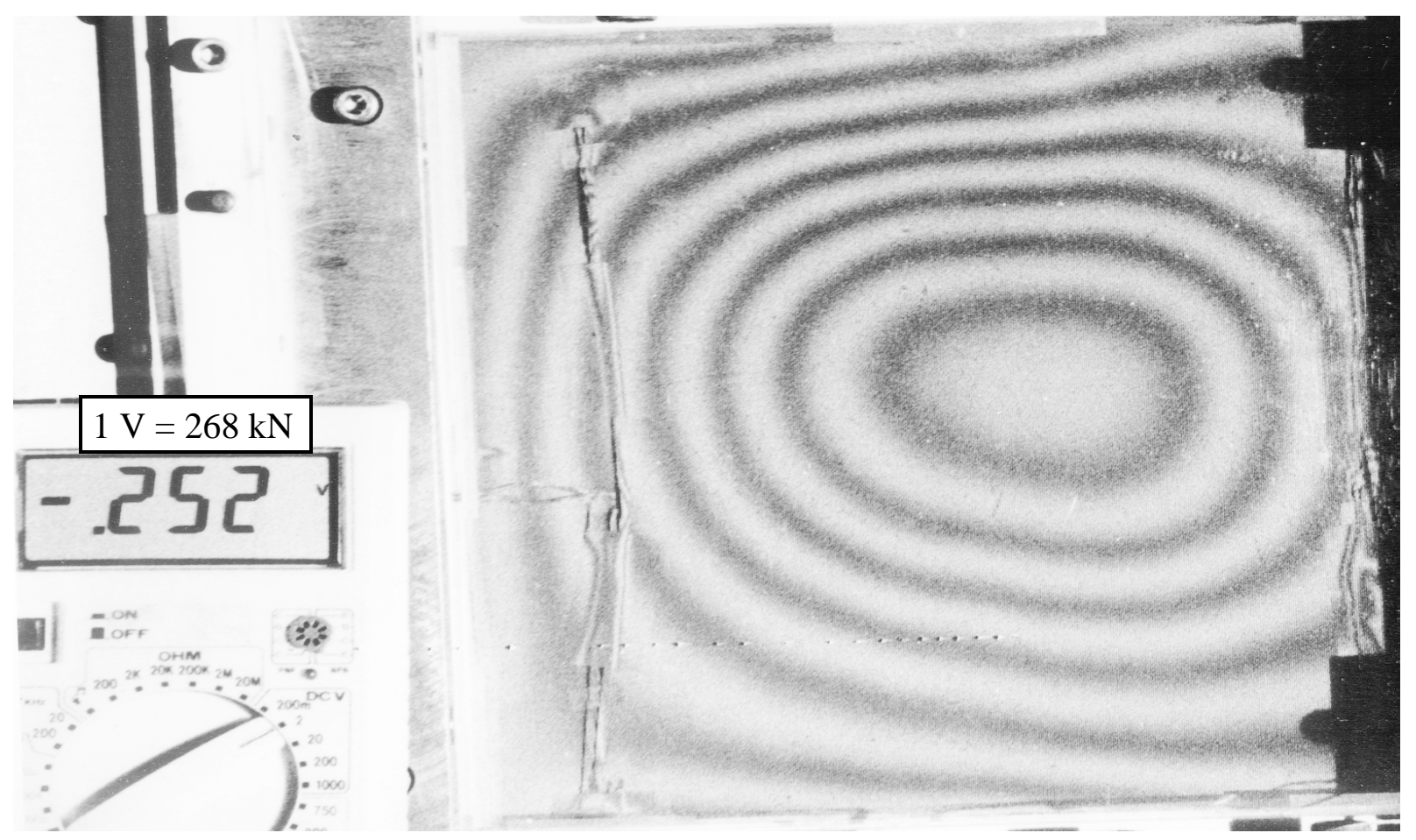

Fig. 5.35: Postbuckling Moire' Fringe Patterns for Out-of-Plane Deflections in Stiffened $\left[ \pm 45 / 90_{2}\right]_{S}$ Plate

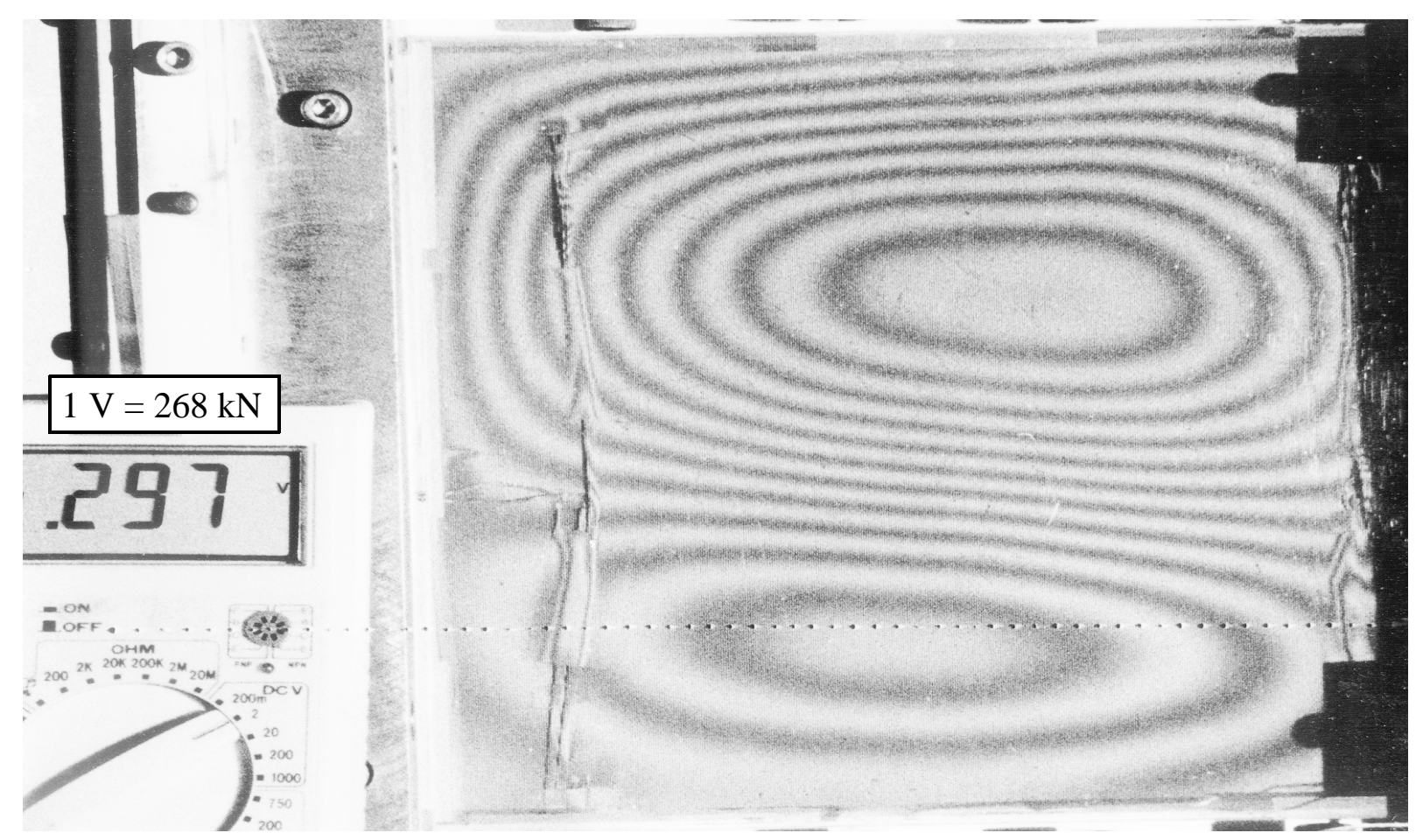

Fig. 5.36: Secondary Buckling Moire' Fringe Patterns for Out-of-Plane Deflections in Stiffened $\left[ \pm 45 / 90_{2}\right]_{\mathrm{S}}$ Plate 


\subsection{CLOSURE}

Six plates were tested in the laboratory to verify the accuracy of the finite element predictions as laid out in Chapter 3. Three of these plates were unstiffened, while the remaining three had stiffeners. Table 5.2 summarizes the experimental data acquired for these plates and compares them with their respective finite element equivalents.

\begin{tabular}{|c|c|c|c|c|c|c|c|}
\hline & & \multicolumn{3}{|c|}{ Unstiffened Plates } & \multicolumn{3}{|c|}{ Stiffened Plates } \\
\hline & & {$\left[ \pm 45 / 0_{2}\right]_{\mathrm{S}}$} & {$[ \pm 45 / 0 / 90]_{\mathrm{S}}$} & {$\left[ \pm 45 / 90_{2}\right]_{\mathrm{s}}$} & {$\left[ \pm 45 / 0_{2}\right]_{\mathrm{s}}$} & {$[ \pm 45 / 0 / 90]_{\mathrm{s}}$} & {$\left[ \pm 45 / 90_{2}\right]_{\mathrm{s}}$} \\
\hline \multirow{4}{*}{$\begin{array}{l}\text { Prebuckling } \\
\text { stiffness (GPa) }\end{array}$} & FEA & 91.6 & 62.2 & 24.9 & 107.0 & 77.5 & 45.5 \\
\hline & $\operatorname{Exp}_{\varepsilon}$ & 93.8 & 58.1 & 23.5 & 102.0 & 80.9 & 40.4 \\
\hline & Exp. $_{\Delta}$ & 87.2 & 61.1 & 24.4 & 88.0 & 75.5 & 45.0 \\
\hline & Exp.av $_{\text {av }}$ & $90.5(-1.2)$ & $59.6(-4.2)$ & $24.0(-3.6)$ & $95.0(-11)$ & $78.2(1.0)$ & $42.7(-6.2)$ \\
\hline \multirow{2}{*}{$\begin{array}{l}\text { Buckling load } \\
(\mathrm{kN})\end{array}$} & FEA & 24.0 & 26.0 & 20.8 & 40.0 & 44.9 & 58.6 \\
\hline & Exp. & $22.0(-8.3)$ & $23.0(-11.5)$ & $21.0(1.0)$ & $37.6(-6.0)$ & $47.0(6.8)$ & $60.0(2.4)$ \\
\hline \multirow{4}{*}{$\begin{array}{l}\text { Postbuckling } \\
\text { stiffness (GPa) }\end{array}$} & FEA & 38.8 & 30.5 & 15.1 & 67.7 & 50.0 & 28.2 \\
\hline & $\operatorname{Exp}_{\varepsilon}$ & 43.3 & 31.6 & 13.8 & 74.1 & 58.2 & 36.2 \\
\hline & $\operatorname{Exp}_{\cdot \Delta}$ & 41.1 & 35.2 & 18.8 & 64.0 & 59.5 & 35.5 \\
\hline & Exp.av $_{\cdot a v}$ & $42.2(8.8)$ & $33.4(9.5)$ & $16.3(7.9)$ & $69.1(2.1)$ & $58.9(17.6)$ & $35.9(27.3)$ \\
\hline \multirow{2}{*}{$\begin{array}{l}\text { Sec. buckling } \\
\text { load }(\mathrm{kN})\end{array}$} & FEA & \multirow{4}{*}{\multicolumn{3}{|c|}{ Not applicable. }} & 130.0 & 105.0 & 68.4 \\
\hline & Exp. & & & & $136.0(4.6)$ & $102.0(-3.8)$ & 79. (15.5) \\
\hline \multirow{2}{*}{$\begin{array}{l}\text { Load drop } \\
(\mathrm{kN})\end{array}$} & FEA & & & & 7.0 & 6.4 & 5.1 \\
\hline & Exp. & & & & $2.0(-71.4)$ & $5.4(-15.6)$ & $1.1(-78.4)$ \\
\hline \multicolumn{8}{|c|}{ 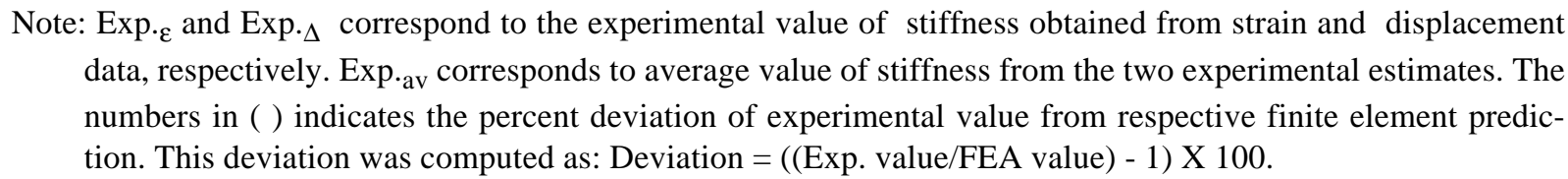 } \\
\hline
\end{tabular}


Based on the data presented in Table 5.2, the following comments can be made:

- The finite element code was able to predict the prebuckling stiffness of both unstiffened and stiffened laminates with a fair degree of accuracy. This is seen through the presence of good correlation between the average of experimentally evaluated stiffness estimates and numerical predictions. Also, it is seen that numerical calculations predicted that the prebuckling stiffness of a plate increased with increasing number of $0^{\circ}$ plies in the plate. This prediction stands corroborated from experimental data. It is also seen that this trend was more pronounced in case of unstiffened panels, since in the stiffened plates stiffeners tended to somewhat attenuate the contribution of lamination sequence to the overall prebuckling stiffness of the plate.

- The agreement between numerical predictions and experimental data for buckling loads of unstiffened and stiffened plates was reasonable. Also, it is seen that the trend in variation in buckling loads due to changes in lamination sequence as obtained from experimental data were similar to those made by finite element calculations. Specifically, for the unstiffened plates the $[ \pm 45 / 0 / 90]_{\mathrm{S}}$ plate had the highest buckling load, and for the stiffened plates the $\left[ \pm 45 / 90_{2}\right]_{\text {s }}$ plate had the highest buckling load.

- Table 5.2 also compares the experimentally observed postbuckling stiffnesses of plates tested in laboratory with numerical predictions. The agreement between these two sets of data was good for unstiffened plates. However, the same was not entirely true for stiffened plates. This difference for the stiffened plates can be attributed to the fact that the stiffened plates buckled at very high loads compared to unstiffened plates. Thus, calculation of postbuckling stiffness from experimental data entailed treading into load ranges exceeding $53.5 \mathrm{kN}$. It has been pointed earlier that at these high loads the signal corresponding to the load channel had a considerable amount of noise. This in turn could be partly responsible for the lack of correlation between experimentally evaluated values of postbuckling stiffness and numerical predictions. We see from Table 5.2 that the degree of disagreement between the numerical and experimental estimates of 
postbuckling stiffness of stiffened plates increased with increasing buckling load. Thus, the disagreement was maximum $(27.3 \%)$ for the $[ \pm 45 / 0 / 90]_{\mathrm{S}}$ stiffened plate, which had the highest buckling load of $60.0 \mathrm{kN}$. Similarly, the disagreement was least $(2.1 \%)$ for the $\left[ \pm 45 / 0_{2}\right]_{\mathrm{S}}$ stiffened plate, which had the least buckling load among stiffened plates. This trend was consistent with the accounting of the aforesaid deviation.

- Comparing the numerical predictions for prebuckling and postbuckling stiffnesses reveals that the drop in postbuckling stiffness was more severe for unstiffened plates than that for unstiffened plates. While the ratio of these two stiffnesses for unstiffened $\left[ \pm 45 / 0_{2}\right]_{\mathrm{S}},[ \pm 45 / 0 / 90]_{\mathrm{S}}$ and $\left[ \pm 45 / 90_{2}\right]_{\mathrm{s}}$ plates, based on numerical calculations, was 0.423 , 0.490, and 0.606, respectively, the ratio of these two stiffnesses for stiffened $\left[ \pm 45 / 0_{2}\right]_{\mathrm{S}}$, $[ \pm 45 / 0 / 90]_{\mathrm{S}}$ and $\left[ \pm 45 / 90_{2}\right]_{\mathrm{S}}$ plates, based on numerical calculations, was $0.633,0.645$ and 0.620, respectively. Once again, these numerical trends were in agreement with experimental results. The relatively low severity of the reduction in postbuckling stiffness for stiffened plates can be attributed to the fact that the stiffeners on these panels remained ever straight due to their proximity to the simply supported edges and hence did not by themselves experience any postbuckling softening.

- The numerical prediction that no secondary buckling will occur in almost square plates if the transverse inplane displacement along the simply supported edges is not sufficiently constrained stands experimentally verified. It is seen through Table 5.2 that the unstiffened plates did not exhibit secondary buckling.

- Finite element analysis predicts that the presence of "sufficiently heavy" stiffeners along the simply supported edges can sufficiently constrain the variation of $v$ displacement along their length. This constraint can, in turn, trigger secondary buckling. This prediction stands experimentally verified, since the stiffened plates were found to experience secondary buckling, which is consistent with finite element predictions. 
- The numerical and experimental values of secondary buckling loads for the stiffened plates tested were in reasonable agreement mutually. Numerical calculations had predicted that the plate with highest inplane longitudinal stiffness would have the highest secondary buckling load. Experimental observations were consistent with this numerical prediction.

The following chapter summarizes the conclusions of the present research effort, and also makes appropriate recommendations for further work in this field. 


\section{CONCLUSIONS AND RECCOMMENDATIONS FOR FURTHER STUDIES}

\subsection{INTRODUCTION}

As detailed in the first chapter of this document, prior to this study not a significantly large amount of research had been conducted in the field of secondary buckling of rectangular composite plates. Further, whatever little research had been done was found wanting in the following aspects:

- A bulk of the research which had been conducted was specific to plates which were simply supported on all of their four edges. The fact that most of the applications of rectangular plates have clamped edges makes their study important.

- The interaction between the boundary conditions of the plate and its secondary buckling characteristics had remained unexplored so far. Whatever work that was done in this context was essentially specific to isotropic plates.

- Very little amount of experimental investigation had been conducted in parallel to numerical investigations. This gap had to be filled, especially because the understanding received as a consequence of diverse numerical and analytical studies of the secondary buckling has been limited in scope and spectrum.

- The question as to how imperfections in plates influence its secondary buckling characteristics had remained unresolved so far. Such imperfections could exist due to geometric or material factors. A typical case of material type of imperfection would be a plate with one of its plies disoriented. A typical case of imperfection rooted in geometric factors could be a small amount of curvature in an otherwise flat plate due to a defective manufacturing process. 
- As mentioned earlier, secondary buckling in composite plates had not been addressed so far in great detail. Further, investigators had usually neglected the influence of bendingtwisting coupling coefficients on account of relatively low magnitudes. However, in view of the probable imperfection sensitivity of panels to secondary buckling loads [24], the roles played by $D_{16}$ and $D_{26}$ merit greater attention.

To address the issues mentioned above, a finite element code was developed to explore the phenomenon of secondary buckling in detail. The choice of finite-element approach was dictated by the fact that this method by itself is powerful enough to handle diverse issues such as boundary conditions, material and geometric imperfections, stacking sequence, stiffener properties, $D_{16}$ and $D_{26}$, etc., in a systematic, orderly, and relatively easy way.

The validity of the code was checked in a systematic way so that the role played by all the elements of the $A, B$, and $D$ stiffness matrices stood individually verified and tested. This code was later used to predict the deep post-buckling behavior of stiffened and unstiffened composite laminates when subjected to different boundary conditions. A large number of conclusions have been arrived at as a consequence of an array of numerical studies conducted in this regard. These conclusions will be summarized later. Also, with a view to validate the predictions of the finite element analysis of these composite laminates, a limited number of experiments were conducted. Specifically, three unstiffened composite plates and three stiffened composite plates were fabricated and subjected to uniform inplane end shortening loads. It was found that experimental data were fairly close to finite element predictions.

\subsection{SUMMARY OF CONCLUSIONS OF NUMERICAL STUDIES}

A variety of numerical studies have been conducted in the present investigation with a view to explore the influence of diverse set of variables on the deep postbuckling and secondary buckling of clamped-simply supported composite plates. These variables include inplane boundary conditions along the simply supported edges of the plate, presence of stiffeners, presence of imperfections, and lamination sequence. A summary of conclusions of these 
parametric studies is given below:

\subsubsection{Influence of Boundary Conditions}

- The presence of restraints on the inplane normal displacement along the simply supported edge of the plate tends to destabilize the plate in the deep postbuckling range. Increasing the degree of restraint in this sense leads to increasing postbuckling instability.

- The most extreme case is the one where the inplane normal displacement is zero along the simply supported edge. In such a situation, secondary buckling occurs at the lowest values of inplane external compressive loads and end shortening. The other extreme corresponds to a situation where the inplane normal displacement is totally unrestrained along the simply supported edge. In such plates, no secondary buckling was found to occur.

\subsubsection{Influence of Stiffeners}

- The probability of the occurrence of secondary buckling in plates increases with increasing inplane bending stiffness of the stiffeners. Thus, while a plate which is very lightly stiffened along the simply supported edges shows no secondary buckling, a plate which has heavier stiffeners undergoes secondary buckling.

- It has been found that plates which do not exhibit secondary buckling have significantly more deformed simply supported edges than those plates which exhibit secondary buckling. In a qualitative sense, it was found that plates which have "sufficiently straight" edges also tend to exhibit secondary buckling.

- The total load borne by stiffened plates for a given value of end shortening is significantly higher than that borne by an unstiffened plate. With the stiffener in close proximity to the simply supported edges, the stiffeners exhibit very little bending and thus are able to bear large compressive loads throughout the loading cycle of the plate. 
- Finally, stiffeners tend to affect the curvature of the plate near the lines of simple support due to their considerable torsional rigidity. Hence, the portion of the stiffened plate near the stiffener appears to remain inplane.

\subsubsection{Influence of Lamination Sequence}

- A plate which is stiff along the loading direction of the plate has a higher value of secondary buckling load than one which is soft. Also, plates which are stiff along the loading direction need higher values of end shortening to trigger secondary buckling.

- The load drop associated with secondary buckling is maximum for the plate which is stiffest in the direction of compressive loading.

\subsubsection{Influence of Imperfections}

- Plates with small magnitudes of out-of-plane imperfections do not have a sharp and well defined primary buckling point. Also for a given value of external load, these plates tend to deflect out-of-plane more than their perfect analogs.

- The imperfections studied here tend to delay the event of secondary buckling. Thus an imperfect plate will exhibit secondary buckling at higher values of end shortening as well as external compressive load than its perfect counterpart.

- The delay in the onset of secondary buckling in imperfect plates is even larger if the plate is a stiffened one.

- While the post-secondary buckled shape of a perfect plate is such that its out-of-plane deflection at center is identically zero, the same is not true of an imperfect plate. The outof-plane deflection at the center of imperfect plates after secondary buckling is not exactly zero. 


\subsubsection{Comparison with Metallic Plates}

- It has been found that, in a qualitative sense, the postbuckling and secondary buckling characteristics for metallic plates are similar to their quasi-isotropic counterparts. The difference in response is merely quantitative in nature. The metallic plates, due to their higher inplane as well as bending stiffnesses, tend to undergo buckling and secondary buckling at higher loads.

\subsubsection{Influence of Out-of-Plane Shear Deformations on Secondary Buckling}

- It has been found that out-of-plane shear strains have negligible impact on the secondary buckling characteristics of composite plates. Thus, a plate with normal through-thickness shear moduli and a plate having 2 orders of magnitude higher value of through-thickness shear moduli have very similar postbuckling and secondary buckling characteristics.

\subsection{SUMMARY OF CONCLUSIONS OF EXPERIMENTAL STUDIES}

As previously stated, three unstiffened and three stiffened plates were tested in the laboratory to augment the confidence in the validity of the numerical predictions made in Chapter 3. A summary of conclusions of these experimental studies is given below.

- The finite element code is able to predict the prebuckling response of both stiffened and unstiffened plates with a fair degree of accuracy. This is seen through the presence of good correlation between the experimental and numerical estimates of plate stiffnesses.

- The agreement between the experimental and numerical predictions for buckling loads of these plates is also reasonable. Also, the finite element code does a good job in tracking the variation in primary buckling loads of composite plates with changing lamination sequences. The numerical code is also able to reasonably predict the postbuckling strain and out-of-plane deflection responses of both stiffened and unstiffened plates. 
- The numerical prediction that no secondary buckling will occur in almost square plates if the transverse inplane displacement along the simply supported edge is nearly free stands experimentally verified. It was seen in the laboratory that the unstiffened plates did not exhibit secondary buckling.

- The finite element prediction that the presence of sufficiently heavy stiffeners along the simply supported edges can trigger the secondary buckling stands corroborated by way of experimental evidence. Also, the numerical predictions for the magnitude of the inplane compressive load at the point of secondary buckling are in reasonable agreement with experimentally observed values of the same.

\subsection{SCOPE FOR FURTHER RESEARCH}

Clearly, the primary recommendation to be made regarding future research it to test a larger number of plates with varying stiffener thickness. In the present research, numerical investigations were carried out for stiffened plates with stiffener thickness of 2.0, 1.8, 1.6, 1.4, 1.2 and $1.0 \mathrm{~mm}$. In the experiments, only a $2.0 \mathrm{~mm}$ thickness was considered. Plates with these other stiffener thicknesses could be tested in a laboratory. Such a testing of plates will yield valuable information regarding the secondary buckling characteristics of stiffened plates, and also about what it takes for a plate to exhibit secondary buckling. Numerical calculations predict that some of these plates will directly go into a buckling mode that will have two half-waves in the loading direction. It would be interesting to see that happen in the laboratory.

The issue of imperfections is a significant one. Present research indicates that the presence of these imperfections could delay the onset of secondary buckling. Experiments on plates which have small amounts of out-of-plane imperfections as a consequence of a misaligned ply will help to confirm these predictions.

Also, all the plates tested in the laboratory so far are almost square in shape. Since practical applications utilize plates of varying aspect ratios, there is a real merit in conducting numerical as well as experimental investigations on secondary buckling of rectangular plates as well. 
Finally, it is known that the event of secondary buckling is essentially a dynamic event. The approach used in this investigation has relied on a static criterion of stability to detect the onset of this event. However, such an approach is not equipped with the potential to determine the intermittent shapes of the plate, i.e., between the two static configurations of the plate. Implementation of a dynamic analysis will greatly help in understanding the transitory states of the plate. Thus, analysis of secondary buckling from a dynamic perspective is indeed recommended.

In summary, there is a considerable need to conduct further studies in this particular area of structural mechanics. The ultimate aim of this research activity should be to evolve a set of standards and guidelines that could aid the designers in better utilizing the postbuckling strengths of plates for purposes of economy and efficiency. 


\section{REFERENCES}

1. Bryan, G. H., 'On the stability of a plane plate under thrusts in its own plane with applications to the 'buckling' of the sides of the ship', Proc. London Math. Soc., v. 22, 54-67, 1891.

2. March, H., 'Buckling of flat plywood plates in compression, shear, or combined compression and shear', Forest Products Lab., Madison, WA, Rept. no. 1316, 1942.

3. Leissa, A. W., 'A review of laminated composite plate buckling', App. Mech. Review, v. 40, no. 5, May 1987, 575-591.

4. Librescu, L., Lin, W., Nemeth, M.P., and Starnes, J.H., 'Thermomechanical postbuckling of geometrically imperfect flat and curved panels taking into account of tangential edge constraints', J. of Thermal Stresses, 18, 465-482, (1995).

5. Librescu, L., and Lin, W., 'Vibration of thermomechanically loaded flat and curved panels taking into account of geometric imperfections and tangential edge restraints', Int. J. Solids Struc., 17, 2161-2181, (1997).

6. Librescu, L., Stability analysis of plates and shells, NASA Technical Report NASA/CP-1998206280, Jan. 1998.

7. Stein, M., The phenomenon of change in buckle pattern in elastic structures, NASA Technical

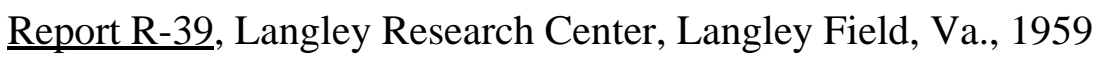

8. Stein, M., Postbuckling behavior of rectangular plates, Ph.D. dissertation, Virginia Polytechnic and State University, June 1958.

9. Stein, M., Loads and deformations of buckled rectangular plates, NASA Technical Report R$\underline{40},(1959)$.

10. Supple, W.J., 'Couple branching configurations in elastic buckling of symmetric structural systems', Int. J. Mech. Sci., 9, 97-112, (1967).

11. Supple, W.J., 'On the change in buckle pattern of elastic structures', Int. J. Mech. Sci., 10, 737-745, (1968).

12. Supple, W.J., 'Changes of wave-form of plates in the postbuckling range', Int. J. Mech. Sci., 6, 1243-1258, (1970).

13. Movchan, A.A., 'The direct method of Liapunov in stability problems of elastic systems', $J$. App. Mathe. Mech., 23, 686-700, (1959). 
14. Stroebel, G.J., and Warner, W.H., 'Stability of secondary bifurcation for von Karman plates', J. of Elasticity, 3, 185-202, (1973).

15. Koiter, W.T., Elastic stability and post-buckling behavior, Proc. Sym. Nonlinear Problems, Ed: R.E. Langer, Univ. of Wisconsin Press, Madison, 257-274, (1963).

16. Fitch, H.R., 'The buckling and post-buckling of spherical caps under concentrated load', Int. J. Solids Struc., 4, 421-446, (1968).

17. Nakamura, T., and Uetani, K., 'The secondary buckling and postbuckling behaviors of rectangular plates', Int. J. Mech. Sci., 21, 265-286, (1979).

18. Maskant, R., and Roorda, J., 'Mode jumping in biaxially compressed plates', Int. J. Solids Struc., 29, 1209-1219, (1992).

19. Uemura, M., and Byon, O-Il, 'Secondary buckling of a flat plate under uniaxial compression, Part 1: Theoretical analysis of simply supported plate', Int. J. nonlinear Mech., 12, 355-370, (1977).

20. Uemura, M., and Byon, O-Il, 'Secondary buckling of a flat plate under uniaxial compression, Part 1: Analysis of clamped plate by F.E.M. and comparison with experiments', Int. J. nonlinear Mech., 13, 1-14, (1978).

21. Carnoy, E.G., 'Finite-element analysis of the secondary buckling of a flat plate under uniaxial compression', Int. J. Nonlinear Mech., 18, 167-175, (1983).

22. Hughes, T.J.R., and Liu, W.K., 'Nonlinear finite-elements of shells, Part I: Three dimensional shells', Comp. Meth. Appl. Mech. Eng., 26, 331-362, (1981).

23. Hughes, T.J.R., and Liu, W.K., 'Nonlinear finite-elements of shells, Part II: Two dimensional shells', Comp. Meth. Appl. Mech. Eng., 27, 167-181, (1981).

24. Shin, D.K., Griffin, O.H. and Gurdal, Z., 'Postbuckling response of laminated plates under uniaxial compression', Int. J. Nonlinear Mech., 28, 95-115, (1993).

25. Stoll, F., 'Analysis of snap phenomenon in buckled plates', Int. J. nonlinear Mech., 29, 123138, (1994).

26. Whitney, J. M., Structural Analysis of Laminated Anisotropic Plates, Technomic, Lancaster, $\mathrm{Pa},(1987)$.

27. Riks, E., Rankin, C.C., and Brogan, F., 'On the solution of mode jumping phenomena in thinwalled structures', Comp. Methods Appl. Mech Engrg, 136, 59-92, (1996). 
28. Chang-jun, C, and Xin-chun, S., 'Mode jumping of simply supported rectangular plates on nonlinear elastic foundation', Int. J. of Nonlinear Mech., 32, 161-172, (1997).

29. Thurston, G.A., 'Continuation of Newton's method through bifurcation points', J. Appl. Mech., 36, 425-430, (1979).

30. Chia, C. Y., Nonlinear Analysis of Plates, McGraw Hill, New York, 96-99, (1980).

31. Crisfield, M. A., Nonlinear Finite Element Analysis of Solids and Structures, Wiley, New York (1991).

32. Reddy, J. N., An Introduction to the Finite Element Method, McGraw Hill, New York, (1993).

33. IMSL Math/Library, Fortran Subroutines for Mathematical Applications, version 2.0, Sept. 1991, IMSL Inc., Houston, Texas.

34. Lee, H. H., and Hyer, M. W., "Postbuckling failure of composite plates with central holes", Center for Composite Materials and Structures, Virginia Polytech. Inst. and State Univ., Blacksburg, VA, Rept. no. CCMS-92-07.

35. Chang, F., "Moire' methods of strain analysis", Manual on Experimental Stress Analysis, 5th ed., edited by Doyle, J. F., and Phillips, J. W., Soc. for Exp. Mech., 1989.

36. Hyer, M. W., Stress Analysis of Fiber Reinforced Composite Materials, WCB/McGraw Hill, New York, (1998). 


\section{APPENDIX}

\section{EXPRESSIONS FOR DIRECT STIFFNESS MATRICES}

Equation 11 in Chapter 2 represents the equilibrium equation of a laminated plate in the three translational directions $(x, y, z)$ and two rotational directions lying in the $x-z$ and $y-z$ planes.

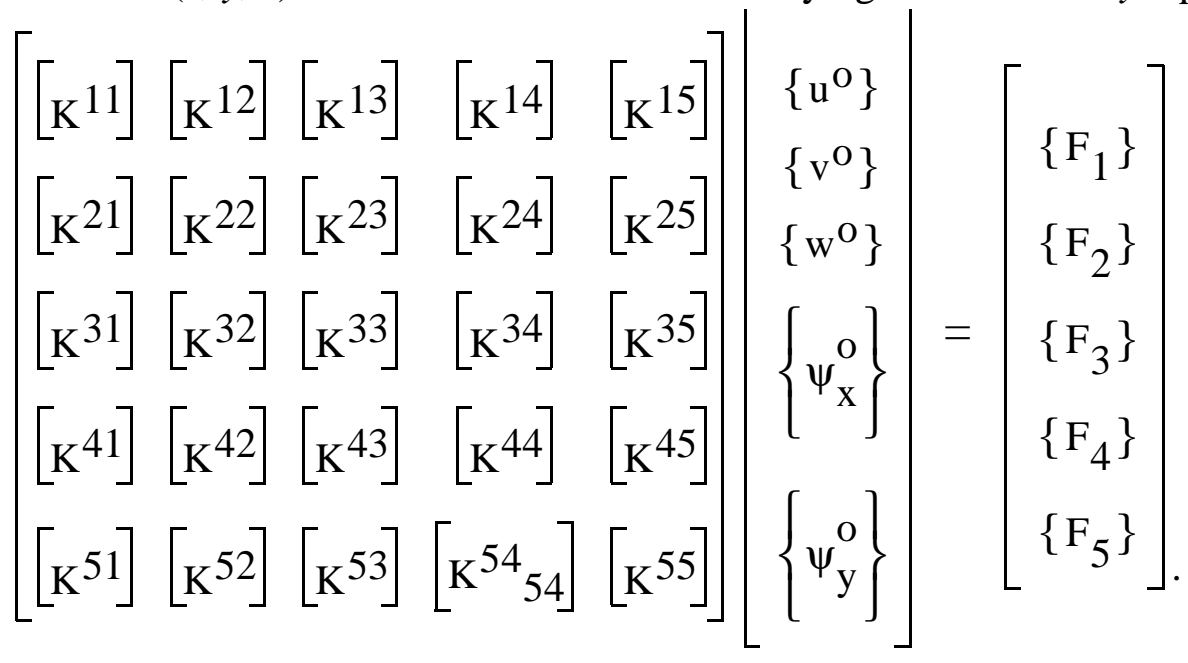

These equations are nonlinear in nature since the sub-matrices $\left[\mathrm{K}^{\mathrm{ij}}\right]$ are dependent on the displacement field $\left(\mathrm{u}^{\mathrm{o}}, \mathrm{v}^{\mathrm{o}}, \mathrm{w}^{\mathrm{o}}, \psi_{\mathrm{x}}^{\mathrm{O}}\right.$, and $\left.\psi_{\mathrm{y}}^{\mathrm{O}}\right)$. The overall matrix $[\mathrm{K}]$, which is an assembly of submatrices $\left[\mathrm{K}^{\mathrm{ij}}\right]$, is called the direct stiffness matrix. Here, subscripts $i$ and $j$ refer to the degrees of freedom of the plate, which are $\mathrm{u}^{\mathrm{o}}, \mathrm{v}^{\mathrm{o}}, \mathrm{w}^{\mathrm{o}}, \psi_{\mathrm{x}}^{\mathrm{o}}$, and $\psi_{\mathrm{y}}^{\mathrm{o}}$. As mentioned in section 2.2, these equations are at the element level. Thus, for each element there are $5 \mathrm{n}$ equilibrium equations, where $n$ is the number of nodes in the element, and 5 corresponds to the number of degrees of freedom of the system. This, in turn, implies that for each node there are five equations of equilibrium. A typical equilibrium equation for node $\xi$ associated with $i^{\text {th }}$ degree of freedom can be written as follows:

$$
\sum_{\eta=1}^{\mathrm{n}}\left(\mathrm{K}_{\xi \eta}^{\mathrm{i} 1} \delta \mathrm{u}_{\eta}^{\mathrm{o}}+\mathrm{K}_{\xi \eta}^{\mathrm{i} 2} \delta \mathrm{v}_{\eta}^{\mathrm{o}}+\mathrm{K}_{\xi \eta}^{\mathrm{i} 3} \delta \mathrm{w}_{\eta}^{\mathrm{o}}+\mathrm{K}_{\xi \eta}^{\mathrm{i} 4} \delta \psi_{\mathrm{x} \eta}^{\mathrm{o}}+\mathrm{K}_{\xi \eta}^{\mathrm{i} 5} \delta \psi_{y \eta}^{\mathrm{o}}\right)=\mathrm{F}_{\xi}^{\mathrm{i}}
$$

In this equation, $\delta u^{o}{ }_{\eta}, \delta v^{o}{ }_{\eta}, \delta w^{o}{ }_{\eta}, \delta \psi^{o}{ }_{x_{\eta}}$, and $\delta \psi^{o}{ }_{y_{\eta}}$ are variations associated with $u^{o}, v^{o}, w^{o}$, $\psi_{x}^{0}$, and $\psi_{y}^{0}$ degrees of freedoms, respectively, for node $\eta$. In the finite element code developed, 
as explained in chapter 2, these variations were assumed to have a similar general shape $\phi_{\eta}$. The procedure for arriving at the expressions of the individual members of the direct stiffness matrix is outlined in section 2.2. Using this approach, the following expressions for individual members of direct-stiffness matrix were developed:

$$
\begin{aligned}
& \mathrm{K}_{\xi \eta}^{11}=\mathrm{A}_{11} \mathrm{~S}_{\xi \eta}^{\mathrm{xx}}+\mathrm{A}_{16}\left(\mathrm{~S}_{\xi \eta}^{\mathrm{xy}}+\mathrm{S}_{\eta \xi}^{\mathrm{xy}}\right)+\mathrm{A}_{66} \mathrm{~S}_{\xi \eta}^{\mathrm{yy}} \\
& \mathrm{K}_{\xi \eta}^{12}=\mathrm{A}_{12} \mathrm{~S}_{\xi \eta}^{\mathrm{xy}}+\mathrm{A}_{16} \mathrm{~S}_{\xi \eta}^{\mathrm{xx}}+\mathrm{A}_{26} \mathrm{~S}_{\xi \eta}^{\mathrm{yy}}+\mathrm{A}_{66} \mathrm{~S}_{\eta \xi}^{\mathrm{xy}}=\mathrm{K}_{\eta \xi}^{21} \\
& \mathrm{~K}_{\xi \eta}^{13}=\mathrm{A}_{11}{ }^{\mathrm{x}} \mathrm{R}_{\xi \eta}^{\mathrm{xx}}+\mathrm{A}_{12}{ }^{\mathrm{y}} \mathrm{R}_{\xi \eta}^{\mathrm{xy}}+\mathrm{A}_{16}\left({ }^{\mathrm{x}} \mathrm{R}_{\xi \eta}^{\mathrm{xy}}+{ }^{\mathrm{x}} \mathrm{R}_{\eta \xi}^{\mathrm{xy}}+{ }^{\mathrm{y}} \mathrm{R}_{\xi \eta}^{\mathrm{xx}}\right)+\mathrm{A}_{26}{ }^{\mathrm{y}} \mathrm{R}_{\xi \eta}^{\mathrm{yy}}+ \\
& \mathrm{A}_{66}\left(\mathrm{x}_{\eta \xi}^{\mathrm{xy}}+{ }^{\mathrm{x}} \mathrm{R}_{\xi \eta}^{\mathrm{yy}}\right)=\mathrm{K}_{\eta \xi^{\prime 2}}^{13} \\
& \mathrm{~K}_{\xi \eta}^{14}=\mathrm{B}_{11} \mathrm{~S}_{\xi \eta}^{\mathrm{xx}}+\mathrm{B}_{16}\left(\mathrm{~S}_{\xi \eta}^{\mathrm{xy}}+\mathrm{S}_{\eta \xi}^{\mathrm{xy}}\right)+\mathrm{B}_{66} \mathrm{~S}_{\xi \eta}^{\mathrm{yy}}=\mathrm{K}_{\eta \xi}^{41} \\
& \mathrm{~K}_{\xi \eta}^{15}=\mathrm{B}_{12} \mathrm{~S}_{\xi \eta}^{\mathrm{xy}}+\mathrm{B}_{16} \mathrm{~S}_{\xi \eta}^{\mathrm{xx}}+\mathrm{B}_{26} \mathrm{~S}_{\xi \eta}^{\mathrm{yy}}+\mathrm{B}_{66} \mathrm{~S}_{\eta \xi}^{\mathrm{xy}}=\mathrm{K}_{\eta \xi}^{51} \\
& K_{\xi \eta}^{22}=A_{22} S_{\xi \eta}^{\mathrm{yy}}+A_{26}\left(S_{\eta \xi}^{x y}+S_{\xi \eta}^{x y}\right)+A_{66} S_{\xi \eta}^{x x} \\
& \mathrm{~K}_{\xi \eta}^{23}=\mathrm{A}_{12}{ }^{\mathrm{x}} \mathrm{R}_{\eta \xi}^{\mathrm{xy}}+\mathrm{A}_{22}{ }^{\mathrm{y}} \mathrm{R}_{\xi \eta}^{\mathrm{yy}}+\mathrm{A}_{26}\left({ }^{\mathrm{y}} \mathrm{R}_{\eta \xi}^{\mathrm{xy}}+{ }^{\mathrm{y}} \mathrm{R}_{\xi \eta}^{\mathrm{xy}}+{ }^{\mathrm{x}} \mathrm{R}_{\xi \eta}^{\mathrm{yy}}\right)+\mathrm{A}_{16}{ }^{\mathrm{x}} \mathrm{R}_{\xi \eta}^{\mathrm{xx}}+ \\
& \mathrm{A}_{66}\left(\mathrm{R}_{\eta \xi}^{\mathrm{xy}}+{ }^{\mathrm{y}_{\xi \eta}} \mathrm{xx}\right)=\mathrm{K}_{\eta \xi^{\prime 2}}^{32} \\
& \mathrm{~K}_{\xi \eta}^{24}=\mathrm{B}_{12} \mathrm{~S}_{\eta \xi}^{\mathrm{xy}}+\mathrm{B}_{26} \mathrm{~S}_{\xi \eta}^{\mathrm{yy}}+\mathrm{B}_{16} \mathrm{~S}_{\xi \eta}^{\mathrm{xx}}+\mathrm{B}_{66} \mathrm{~S}_{\xi \eta}^{\mathrm{xy}}=\mathrm{K}_{\eta \xi}^{42} \\
& \mathrm{~K}_{\xi \eta}^{25}=\mathrm{B}_{22} \mathrm{~S}_{\xi \eta}^{\mathrm{yy}}+\mathrm{B}_{26}\left(\mathrm{~S}_{\xi \eta}^{\mathrm{xy}}+\mathrm{S}_{\eta \xi}^{\mathrm{xy}}\right)+\mathrm{B}_{66} \mathrm{~S}_{\xi \eta}^{\mathrm{xx}}=\mathrm{K}_{\eta \xi}^{51}
\end{aligned}
$$




$$
\begin{aligned}
& \mathrm{K}_{\xi \eta}^{33}=\mathrm{A}_{55} \mathrm{~S}_{\xi \eta}^{\mathrm{xx}}+\mathrm{A}_{45}\left(\mathrm{~S}_{\eta \xi}^{\mathrm{xy}}+\mathrm{S}_{\xi \eta}^{\mathrm{yx}}\right)+\mathrm{A}_{44} \mathrm{~S}_{\xi \eta}^{\mathrm{yy}}+ \\
& \frac{1}{2} \int_{\Omega_{\mathrm{e}}}\left[\overline{\mathrm{N}}_{1} \frac{\partial \phi_{\xi} \partial \phi_{\eta}}{\partial \mathrm{x}} \frac{\overline{\mathrm{N}}_{6}}{\partial \mathrm{x}}\left(\frac{\partial \phi_{\xi} \partial \phi_{\eta}}{\partial \mathrm{x}} \frac{\partial \phi_{\xi} \partial \phi_{\eta}}{\partial \mathrm{y}}+\frac{\partial}{\partial \mathrm{y}} \frac{\partial \phi_{\xi} \partial \phi_{\eta}}{\partial \mathrm{x}}\right)+\overline{\mathrm{N}}_{2} \frac{\partial \mathrm{y}}{\partial \mathrm{y}}\right] \mathrm{dxdy} \\
& \mathrm{K}_{\xi \eta}^{34}=\mathrm{A}_{55} \mathrm{~S}_{\xi \eta}^{\mathrm{xo}}+\mathrm{A}_{45} \mathrm{~S}_{\xi \eta}^{\mathrm{yo}}+\mathrm{B}_{11}{ }^{\mathrm{x}} \mathrm{R}_{\xi \eta}^{\mathrm{xx}}+\mathrm{B}_{12}{ }^{\mathrm{y}} \mathrm{R}_{\eta \xi}^{\mathrm{xy}}+ \\
& B_{16}\left({ }^{x}{ }_{\xi \eta}^{x y}+{ }^{x} R_{\eta \xi}^{x y}+{ }^{y} R_{\xi \eta}^{x x}\right)+B_{26}{ }^{y} R_{\xi \eta}^{y y}+B_{66}\left({ }_{R}{ }_{\eta \xi}^{y y}+{ }_{R} R_{\xi \eta}^{x y}\right) \\
& \mathrm{K}_{\xi \eta}^{35}=\mathrm{A}_{45} \mathrm{~S}_{\xi \eta}^{\mathrm{xo}}+\mathrm{A}_{44} \mathrm{~S}_{\xi \eta}^{\mathrm{yo}}+\mathrm{B}_{12}{ }^{\mathrm{x}} \mathrm{R}_{\xi \eta}^{\mathrm{xy}}+\mathrm{B}_{22}{ }^{\mathrm{y}} \mathrm{R}_{\xi \eta}^{\mathrm{yy}}+ \\
& { }^{B}{ }_{16}{ }^{x} R_{\xi \eta}^{x x}+B_{26}\left({ }_{R}{ }_{\eta \xi}^{x y}+{ }_{R} R_{\xi \eta}^{y y}+{ }_{R_{\xi \eta}}^{x y}\right)+B_{66}\left(R_{\eta \xi}^{x y}+{ }^{y_{k}} R_{\xi \eta}^{x x}\right) \\
& \mathrm{K}_{\xi \eta}^{43}=\mathrm{A}_{55} \mathrm{~S}_{\eta \xi}^{\mathrm{xo}}+\mathrm{A}_{45} \mathrm{~S}_{\eta \xi}^{\mathrm{yo}}+\frac{1}{2}\left(\mathrm{~B}_{11}{ }^{\mathrm{x}} \mathrm{R}_{\eta \xi}^{\mathrm{xx}}+\mathrm{B}_{12}{ }^{\mathrm{y}} \mathrm{R}_{\xi \eta}^{\mathrm{xy}}\right)+
\end{aligned}
$$

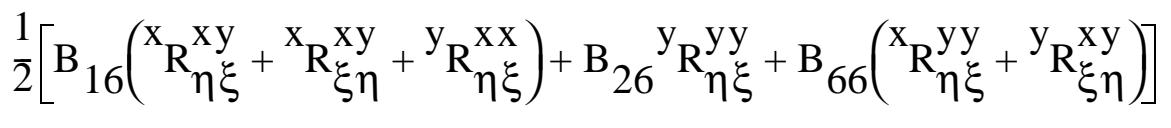

$$
\begin{aligned}
& \mathrm{K}_{\xi \eta}^{44}=\mathrm{D}_{11} \mathrm{~S}_{\xi \eta}^{\mathrm{xx}}+\mathrm{D}_{16}\left(\mathrm{~S}_{\xi \eta}^{\mathrm{xy}}+\mathrm{S}_{\eta \xi}^{\mathrm{xy}}\right)+\mathrm{D}_{66} \mathrm{~S}_{\xi \eta}^{\mathrm{yy}}+\mathrm{A}_{55} \mathrm{~S}_{\xi \eta}^{\mathrm{oo}} \\
& \mathrm{K}_{\xi \eta}^{45}=\mathrm{D}_{12} \mathrm{~S}_{\xi \eta}^{\mathrm{xy}}+\mathrm{D}_{16} \mathrm{~S}_{\xi \eta}^{\mathrm{xx}}+\mathrm{D}_{26} \mathrm{~S}_{\xi \eta}^{\mathrm{yy}}+\mathrm{D}_{66} \mathrm{~S}_{\eta \xi}^{\mathrm{xy}}+\mathrm{A}_{45} \mathrm{~S}_{\xi \eta}^{\mathrm{oo}}=\mathrm{K}_{\eta \xi}^{54} \\
& \mathrm{~K}_{\xi \eta}^{53}=\mathrm{A}_{45} \mathrm{~S}_{\eta \xi}^{\mathrm{xo}}+\mathrm{A}_{44} \mathrm{~S}_{\eta \xi}^{\mathrm{yo}}+\frac{1}{2}\left(\mathrm{~B}_{11} \mathrm{R}_{\eta \xi}^{\mathrm{xy}}+\mathrm{B}_{12} \mathrm{P}_{\eta \xi}^{\mathrm{y}} \mathrm{y}_{\eta}\right)+ \\
& \frac{1}{2}\left[\mathrm{~B}_{16}{ }^{\mathrm{x}} \mathrm{R}_{\eta \xi}^{\mathrm{xx}}+\mathrm{B}_{26}\left(\mathrm{P}_{\xi \eta}^{\mathrm{xy}}+{ }^{\mathrm{x}} \mathrm{R}_{\eta \xi}^{\mathrm{yy}}+{ }^{\mathrm{y}} \mathrm{R}_{\eta \xi}^{\mathrm{xy}}\right)+\mathrm{B}_{66}\left(\mathrm{R}_{\xi \eta}^{\mathrm{xy}}+{ }^{\mathrm{y}} \mathrm{R}_{\eta \xi}^{\mathrm{xx}}\right)\right] \\
& \mathrm{K}_{\xi \eta}^{55}=\mathrm{D}_{22} \mathrm{~S}_{\xi \eta}^{\mathrm{yy}}+\mathrm{D}_{26}\left(\mathrm{~S}_{\eta \xi}^{\mathrm{xy}}+\mathrm{S}_{\xi \eta}^{\mathrm{yx}}\right)+\mathrm{D}_{66} \mathrm{~S}_{\xi \eta}^{\mathrm{xx}}+\mathrm{A}_{44} \mathrm{~S}_{\xi \eta}^{\mathrm{oo}}
\end{aligned}
$$


where,

$$
\begin{gathered}
\mathrm{S}_{\xi \eta}^{\alpha \beta}=\int_{\Omega_{\mathrm{e}}} \frac{\partial \phi_{\xi} \partial \phi_{\eta}}{\partial \alpha} \frac{\partial \beta}{\partial x d y} \\
\mathrm{~S}_{\xi \eta}^{\mathrm{oo}}=\int_{\Omega_{\mathrm{e}}} \phi_{\alpha} \phi \mathrm{dxdy} \\
\alpha_{\mathrm{R}_{\xi \eta}^{\beta \gamma}}^{\beta \gamma}=\int_{\Omega_{\mathrm{e}}} \frac{\partial \mathrm{w}}{2} \frac{\partial \phi_{\xi}}{\partial \alpha} \frac{\partial \phi}{\partial \gamma}
\end{gathered}
$$

where $\alpha, \beta=0, x, y$;

$\mathrm{g}=\mathrm{x}, \mathrm{y}$

and $\Omega_{\mathrm{e}}$ is the area of the finite element.

and,

$$
\begin{aligned}
& \overline{\mathrm{N}}_{1}=\mathrm{A}_{11}\left(\frac{\partial \mathrm{w}}{\partial \mathrm{x}}\right)^{2}+\mathrm{A}_{12}\left(\frac{\partial \mathrm{w}}{\partial \mathrm{y}}\right)^{2}+2 \mathrm{~A}_{16} \frac{\partial \mathrm{w}}{\partial \mathrm{x}} \frac{\partial \mathrm{w}}{\partial \mathrm{y}} \\
& \overline{\mathrm{N}}_{2}=\mathrm{A}_{12}\left(\frac{\partial \mathrm{w}}{\partial \mathrm{x}}\right)^{2}+\mathrm{A}_{22}\left(\frac{\partial \mathrm{w}}{\partial \mathrm{y}}\right)^{2}+2 \mathrm{~A}_{26} \frac{\partial \mathrm{w}}{\partial \mathrm{x}} \frac{\partial \mathrm{w}}{\partial \mathrm{y}} \\
& \overline{\mathrm{N}}_{6}=\mathrm{A}_{16}\left(\frac{\partial \mathrm{w}}{\partial \mathrm{x}}\right)^{2}+\mathrm{A}_{26}\left(\frac{\partial \mathrm{w}}{\partial \mathrm{y}}\right)^{2}+2 \mathrm{~A}_{66} \frac{\partial \mathrm{w}}{\partial \mathrm{x}} \frac{\partial \mathrm{w}}{\partial \mathrm{y}}
\end{aligned}
$$




\section{VITA}

Nachiketa Tiwari was born on November 2, 1966 in Gonda, UP, India. He attended St. Xavier High School in Jaipur, Rajasthan, India. He earned his undergraduate degree in Mechanical Engineering in 1988 from Malaviya Regional Engineering College, Jaipur, India. Thereafter, in 1989 he joined the Indian Institute of Technology, Kanpur, India as a graduate student of Mechanical Engineering. He graduated from that insitution in 1991 with a Master of Technology degree in Mechanical Engineering, with specialization in solid mechanics. In August of 1991 he enrolled at Virginia Polytechnic Institute and State University, Blacksburg, Virginia, as a doctoral student of Engineering Mechanics. In November of 1996, he took a leave of absence from his doctoral program to work for Parametric Technology Corporation, Waltham, Massachusetts as a benchmark applications engineer. In May, 1998, he joined Bose Corporation, Framingham, Massachusetts as an advanced development engineer. He fulfilled his requirements for the degree of Doctor of Philosophy of Engineering Mechanics in May 1999, and currently continues to be employed at Bose Corporation. 